\title{
Holographic Aspects of Chaos and Integrability in String- and M-Theory
}

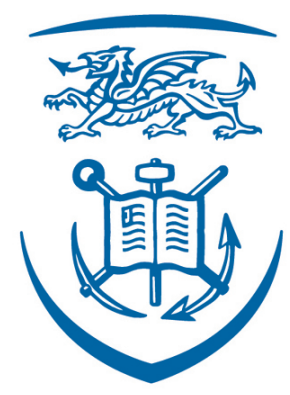

\section{Swansea University Prifysgol Abertawe}

\author{
JEROEN VAN GORSEL \\ Department of Physics \\ Swansea University \\ 2019
}

Submitted to Swansea University in fulfillment of the requirements for the degree of

Doctor of Philosophy 


\section{ABstraCt}

In this thesis we investigate classical integrability of the string worldsheet on different supergravity backgrounds. We focus in particular on the class of half-supersymmetric $\mathrm{AdS}_{7}$ solutions of Massive Type IIA supergravity, that are thought to be the near-horizon limit of a D6-D8NS5 Hanany-Witten brane set-up, and are dual to six-dimensional conformal field theories with $\mathcal{N}=(1,0)$ supersymmetry. We use both analytical and numerical methods to show the (bosonic sector of the) string worldsheet is non-integrable on most of these backgrounds. The backgrounds on which the string is integrable are an infinite massless solution (corresponding to an infinite constant quiver), and a background corresponding to an infinite linear quiver theory.

In addition we find that the (bosonic sector of the) string is integrable on a background that we call $\mathrm{AdS}_{7} \times\left(\mathrm{S}^{3}\right)_{\lambda}$. For this background we show that it corresponds to a $6 \mathrm{~d} \mathrm{SCFT}$ with an infinitely long quiver with an infinite number of flavour groups, all proportional to the colour groups. We study this particular supergravity background in detail, and suggest it corresponds to the large- $N$ limit of the dual SCFT in the limit where the Chern-Simons level $k$ goes to infinity.

This integrable $\mathrm{AdS}_{7} \times\left(\mathrm{S}^{3}\right)_{\lambda}$ background can be obtained as the $\lambda$-deformation of $\mathrm{AdS}_{7} \times \mathrm{S}^{3}$. In this context we study integrable deformations of supergravity backgrounds in the last part of this thesis, in particular non-Abelian T-duality. We present another background on which the string is integrable by performing two non-Abelian T-dualities on two three-spheres inside the $\mathrm{AdS}_{5} \times \mathrm{S}^{5}$ solution and study the resulting background. 


\section{Declaration}

This work has not previously been accepted in substance for any degree and is not being concurrently submitted for any degree.

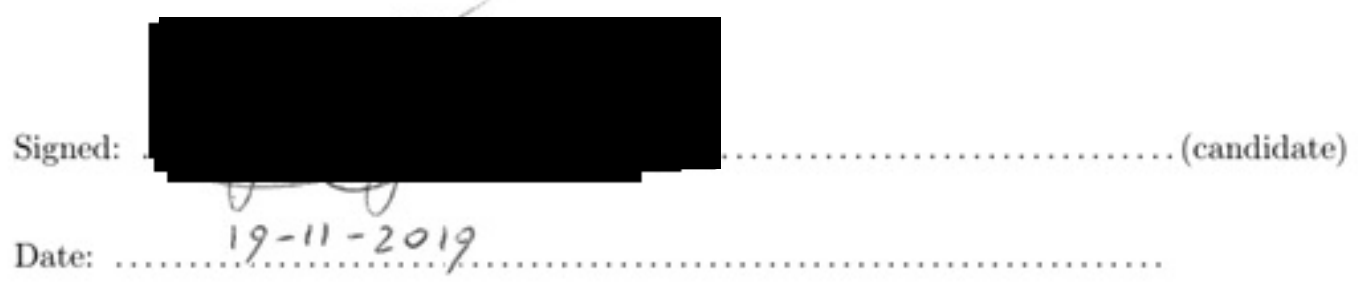

\section{Statement 1}

This thesis is the result of my own investigations, except where otherwise stated. Where correction services have been used, the extent and nature of the correction is clearly marked in a footnote(s). Other sources are acknowledged by footnotes giving explicit references. A bibliography is appended.

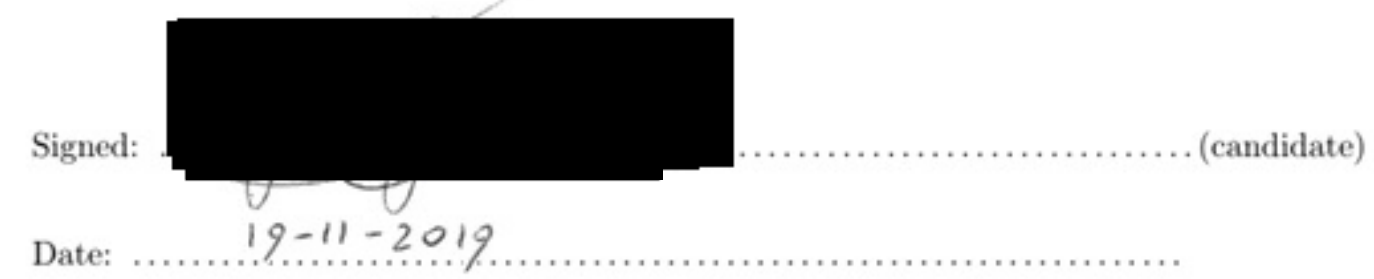

\section{Statement 2}

I hereby give consent for my thesis, if accepted, to be available for photocopying and for interlibrary loan, and for the title and summary to be made available to outside organisations.

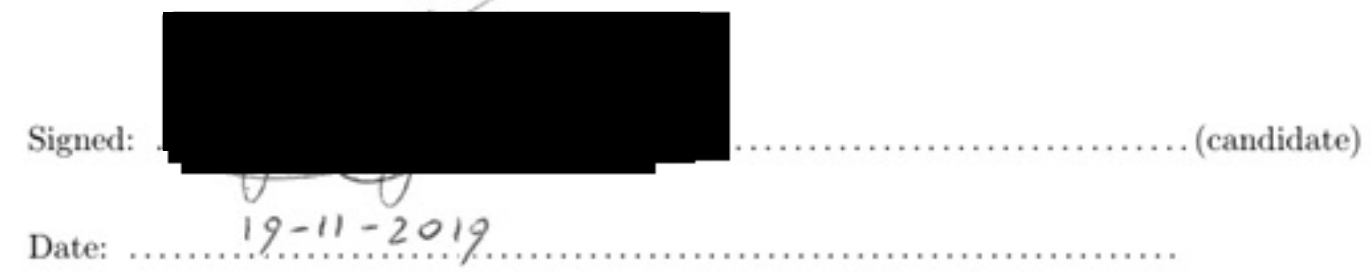




\section{Publications}

The original contributions in this thesis are based on the following (forthcoming) publications:

- J. van Gorsel, The Type IIA Superstring on $A d S_{7} \times\left(\mathrm{S}^{3}\right)_{\lambda}$.

- J. van Gorsel, Infinite Quivers and Continuous Rank Functions.

- K. Filippas, C. Núñez and J. van Gorsel, Integrability and Holographic Aspects of SixDimensional $\mathcal{N}=(1,0)$ Superconformal Field Theories, Journal of High Energy Physics (JHEP), 1906 (2019) 069.

- C. Núñez, J. Manuel Penín, D. Roychowdhury and J. van Gorsel, The non-Integrability of Strings in Massive Type IIA and their Holographic duals, Journal of High Energy Physics (JHEP), 1806 (2018) 078.

- J. van Gorsel and S. Zacarías, A Type IIB Matrix Model via non-Abelian T-dualities, Journal of High Energy Physics (JHEP), 1712 (2017) 101.

I state that this work has not been previously accepted in substance for any degree, nor is it currently submitted in candidature for any degree.

I state that this thesis is the result of my own investigations, except where otherwise stated. Other sources are acknowledged by footnotes giving explicit references. A bibliography is appended.

Jeroen van Gorsel

19 November 2019 


\section{Acknowledgements}

First and foremost I would like to thank my supervisor Carlos Núñez. Your broad range of research interests and excitement to start working on new topics has been a true inspiration. Thank you for the many enjoyable discussions and Skype conversations, and your guidance since the beginning of our collaboration. I learned more over the past three years than I thought would have been possible at the beginning.

Equally important has been the continuing support of my family, and Sabrina Hsu, who helped a great deal to keep me sane during my stay in Swansea. You all helped to make this possible.

I would also like to thank the many staff members, postdocs and other PhD students in the Department of Physics at Swansea University. As a beginning PhD student I benefited immensely from the lectures and journal clubs we organised with Adi Armoni, Prem Kumar, Graham Shore and Timothy Hollowood. During my first year I also learned an incredible amount from the many talks I had with Salomón Zacarías (often over a beer and some spicy Mexican food on the beach).

I am grateful to Daniel Thompson, Timothy Hollowood, Dafydd Price, Sibylle Driezen, and Calan Appadu for many illuminating discussions about integrability, and to Salomón Zacarías, Dibakar Roychowdhury, José Manuel Penín, Kostas Filippas, Prem Kumar, and Stanislav Stratiev for very enjoyable collaborations.

The 'coffee hours' with Connor Barlow Myers, John Godwin, Ogan Özsoy, and Guy Jehu always were a very welcome distraction during the weeks, and a nice excuse to have lengthy discussions on a wide range of topics.

Special thanks go out to my friends in the Netherlands. In particular a group of illustrious night-owl academics that dwell around Geversstraat 63B in Oegstgeest and call themselves the 'Preneurs'.

At last I would like to express special thanks to Alessandro Torrielli and Timothy Hollowood for giving me many valuable detailed comments on the first version of this thesis. 


\section{Contents}

1 Introduction $\quad 1$

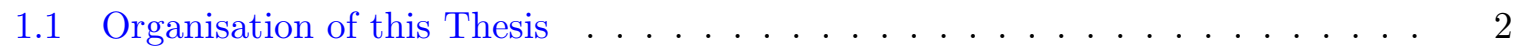

2 Integrability and Superstring Theory 5

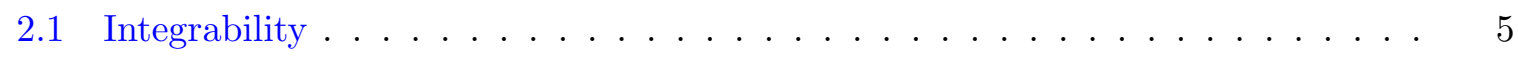

2.1 .1 Integrable Field Theories _ . . . . . . . . . . . . . 7

2.1 .2 Integrability in String Theory . . . . . . . . . . . . . . . . . . . 8

2.2 Principal Chiral Model . . . . . . . . . . . . . . . . . . . . . . . . . . . . 10

2.2.1 Coset Sigma Model . . . . . . . . . . . . . . . . . . . 13

2.2.2 Symmetric Cosets and $\mathbb{Z}_{2}$ grading . . . . . . . . . . . . . . 14

2.3 Wess-Zumino-Witten Model . . . . . . . . . . . . . . . . . . . . 17

2.4 The Green-Schwarz Superstring . . . . . . . . . . . . . . . . . . . . . 19

2.5 Supermanifolds . . . . . . . . . . . . . . . . . . . . . . 19

2.6 Supergroups . . . . . . . . . . . . . . . . . . . . . . . . . . . . . . . . . . . .

2.6.1 Maurer-Cartan form on a Supergroup . . . . . . . . . . . . . 23

2.6 .2 PCM on a Supergroup . . . . . . . . . . . . . . . 24

2.6.3 Graded Supergroups . . . . . . . . . . . . . . . . 25

2.6.4 WZW-model on Supergroups . . . . . . . . . . . . . . . . . . . . . . . . . . . . . 26

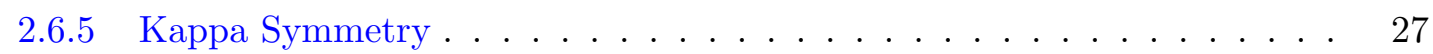

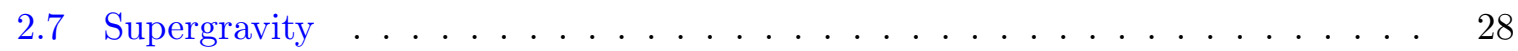

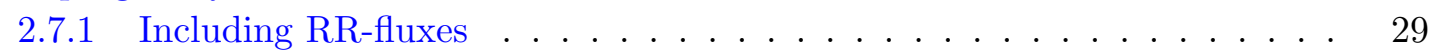

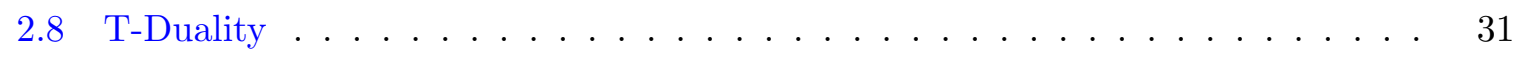

2.8.1 The Buscher Rules . . . . . . . . . . . . . . . . . 31

2.8.2 Transformation of the RR-fluxes . . . . . . . . . . . . . 34

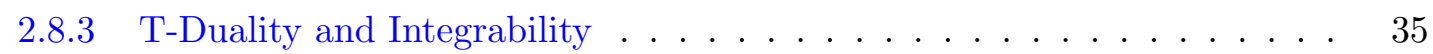

2.9 Non-Abelian T-duality . . . . . . . . . . . . . . . . . . . . . . . . . . . . . . . . . .

2.9 .1 Example: NATD on $\mathrm{SU}(2) \quad \ldots \ldots \ldots \ldots \ldots$

2.10 T-duality at the Quantum Level . . . . . . . . . . . . . . . . . . 39 
3 AdS/CFT and Integrability for Half-Supersymmetric SCFTs 43

3.1 Introduction . . . . . . . . . . . . . . . . . . . . 43

3.2 D-Branes in String Theory . . . . . . . . . . . . . . . . . . . 44

3.2.1 Worldvolume Theories of D-Branes . . . . . . . . . . . . . 45

3.2 .2 P-Branes in Supergravity . . . . . . . . . . . . . . . . . 46

3.3 The AdS/CFT Correspondence . . . . . . . . . . . . . . . . . . . 46

3.3 .1 Open Strings on $\mathrm{AdS}_{5} \times \mathrm{S}^{5} \ldots \ldots \ldots \ldots \ldots \ldots$

$3.3 .2 \mathcal{N}=4$ Super Yang-Mills . . . . . . . . . . . . . . . . . . 49

3.3.3 The AdS/CFT Correspondence . . . . . . . . . . . . . . . . . 49

3.4 Integrability and AdS/CFT . . . . . . . . . . . . . . . . 50

3.5 Half-Supersymmetric Worldvolume Theories . . . . . . . . . . . . . . . . 52

3.5.1 Consistency Condition at the Conformal Fixed Point . . . . . . . . . 54

3.5 .2 Supergravity Limit . . . . . . . . . . . . . . . . . 55

3.6 Half-BPS $\mathrm{AdS}_{p+1}$ Geometries . . . . . . . . . . . . . . . . . . 57

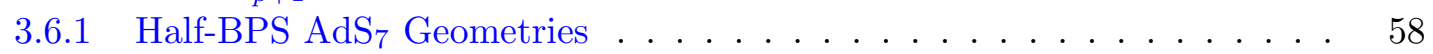

3.6 .2 Half-BPS AdS Geometries . . . . . . . . . . . . . . . . . 59

3.6.3 Notes on a class of Quarter-BPS $\mathrm{AdS}_{3}$ Geometries . . . . . . . . . . . . 60

3.6 .4 Half-BPS $\mathrm{AdS}_{4}$ Geometries . . . . . . . . . . . . . . . . . 61

3.6.5 Half-BPS $\mathrm{AdS}_{6}$ Geometries . . . . . . . . . . . . . . . . 62

3.7 Integrability in $\frac{1}{2}$ BPS Supergravity Solutions . . . . . . . . . . . . 63

$4 \mathbf{A d S}_{7}$ duals of $\mathbf{6 d} \mathcal{N}=(1,0)$ SCFTs $\quad \mathbf{6 7}$

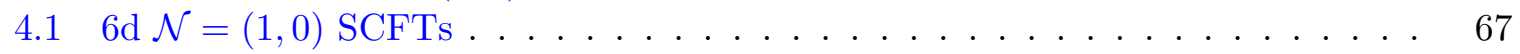

4.1.1 Non-Lagrangian Conformal Fixed Point . . . . . . . . . . . . . 70

4.2 Holographic $\mathrm{AdS}_{7}$ Description . . . . . . . . . . . . . . . . 71

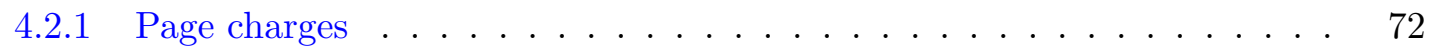

4.2 .2 Linking numbers . . . . . . . . . . . . . . . . . . . . 74

4.3 Connecting the holographic background with the CFT . . . . . . . . . 75

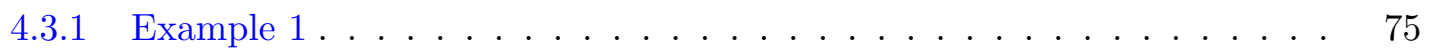

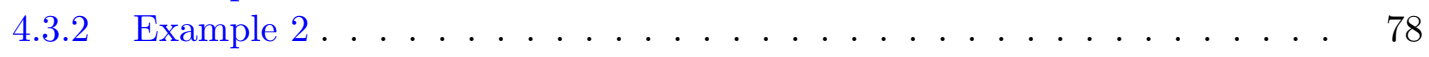

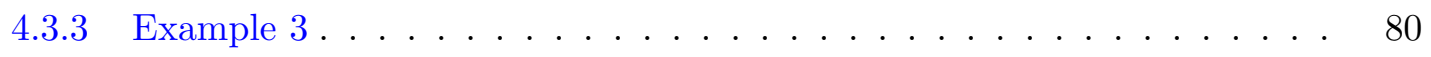

4.3.4 Most generic example . . . . . . . . . . . . . . 82

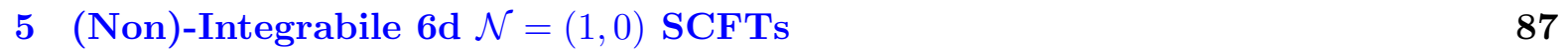

5.1 Introduction . . . . . . . . . . . . . . . . . . 87

5.2 Classical Equations of Motion for String Embeddings . . . . . . . . . . . . . 88

5.3 Analytical (non)-Integrability . . . . . . . . . . . . . . . . . . . . . . . . . . . . . . .

5.3 .1 Kovacic's Algorithm . . . . . . . . . . . . . . . . . . . . . 91

5.3 .2 Kovacic Analysis . . . . . . . . . . . . . . . . . . . . . . . . 92

5.3 .3 Results for Various $\mathrm{AdS}_{7}$ Backgrounds . . . . . . . . . . . . . 97

viii 
5.4 Numerical Indicators of Chaos _. . . . . . . . . . . . . . . . . . 100

5.4 Power Spectra . . . . . . . . . . . . . . . . . . 100

5.4 .2 Poincaré Sections and KAM Tori . . . . . . . . . . . . . . . 100

5.4 Lyapunov Exponents . . . . . . . . . . . . . . . . . . . 103

5.5 Analysis for Various $\mathrm{AdS}_{7}$ Backgrounds . . . . . . . . . . . . . . . . 103

5.5.1 Analysis for Generic Quivers . . . . . . . . . . . . . . 105

5.5.2 Analysis for a Linear Quiver . . . . . . . . . . . . . . . . . . . . . 108

5.5.3 Analysis for the Massless Solution . . . . . . . . . . . . . . . . 110

5.5 .4 Vanishing Warp Factor . . . . . . . . . . . . . . . . 116

5.6 Appendix: Numerical Computation of the Lyapunov Spectrum . . . . . . . . . 120

$6 \quad$ Integrable strings on $\mathbf{A d S}_{7} \times\left(S^{3}\right)_{\lambda} \quad 123$

$6.1 \mathrm{AdS}_{7}$ Geometry for $\alpha(z)=A \sin \omega z \ldots \ldots \ldots \ldots \ldots \ldots$

6.2 Integrability of the Bosonic String . . . . . . . . . . . . . . . . 125

6.3 Relation with $\lambda$-deformation . . . . . . . . . . . . . . . 126

6.3.1 Relation with the NATD of $\mathrm{AdS}_{7} \times \mathrm{S}^{3} \ldots \ldots \ldots \ldots . \ldots \ldots$

6.4 The Type IIA Superstring on $\mathrm{AdS}_{7} \times\left(S^{3}\right)_{\lambda} \ldots \ldots \ldots \ldots \ldots \ldots$

6.5 Continuous Rank Functions and Infinite Quivers . . . . . . . . . . . . . . 129

6.5.1 Defining Quivers from their Flavour Groups . . . . . . . . . . . . . . 129

6.5 .2 Parabolic Quiver . . . . . . . . . . . . . . . . . . . . 130

6.5.3 Sinusoidal Quiver . . . . . . . . . . . . . . . . 133

6.6 Smeared Branes . . . . . . . . . . . . . . . . . . . . 136

6.6.1 Orthogonal Bases of Quivers . . . . . . . . . . . . . . 138

6.7 Field Theory Interpretation of the $\mathrm{AdS}_{7} \times\left(\mathrm{S}^{3}\right)_{\lambda}$ Background . . . . . . . . . . 139

6.7.1 Large Chern-Simons Level . . . . . . . . . . . . . . . . . . . . . . 140

6.7 .2 Central Charge . . . . . . . . . . . . . . . . . . . . . 140

6.7.3 Entanglement Entropy . . . . . . . . . . . . . . . . . . . . 141

6.7 .4 Wilson Loops . . . . . . . . . . . . . . . . . . . 143

7 T-Duality as a Solution Generating Technique $\quad 147$

7.0.1 $\mathrm{AdS}_{5} \times \mathrm{S}^{5}$ Solution . . . . . . . . . . . . . . . . . . 148

7.0.2 NATD inside $S^{5}$ (The Sfetsos-Thompson Solution) . . . . . . . . . . 149

$7.0 .3 \quad$ NATD inside $A d S_{5} \ldots \ldots \ldots \ldots \ldots \ldots$. . . . . . . . . . . 150

7.1 The Double NATD of $\mathrm{AdS}_{5} \times \mathrm{S}^{5} \ldots \ldots \ldots \ldots \ldots \ldots \ldots$

7.1.1 Supersymmetry of the solution . . . . . . . . . . . . . 153

7.1 .2 Quantised Charges ... . . . . . . . . . . . . . 155

7.1 .3 Dielectric branes . . . . . . . . . . . . . . . . . . 159 


\section{INTRODUCTION}

\section{Chapter 1}

\section{Introduction}

In the early 90's research in (super)string theory blossomed and lead to the study of D-branes (planes on which strings can end), whose fluctuations at low energies look like higher dimensional quantum field theories. This lead to a completely new framework in mathematical physics, where one could now understand (supersymmetric) quantum field theories in terms of these D-branes. This allowed many of the developments in mathematical physics that were made over the decades prior (such as dualities between different field theories) to now be understood conceptually in terms of D-branes.

About the same time it came to be understood that the strings and D-branes one studies in superstring theory should be thought of as various limits of two dimensional surfaces (so called M2-branes), that can end on other M2-branes or on M5-branes. Unfortunately these M-theory branes remain at present much less understood. Various mathematical structures that are needed to describe the physics of M-theory remain difficult to work out and seem to require the introduction of new and more general mathematics. The most infamous example of this is the enigmatic six-dimensional $\mathcal{N}=(2,0)$ theory, that is thought to describe the low energy fluctuations of a stack of M5-branes with M2-branes stretching between them. Not only does this field theory contain 'higher' gauge fields (for which it is yet conceptually unclear how their field strength should be constructed in the non-Abelian case), it is also thought this theory cannot be formulated in terms of quantum corrections on top of a classical Lagrangian description.

In an attempt to learn more about six-dimensional theories, we study six-dimensional $\mathcal{N}=(1,0)$ theories in this thesis. The main contribution of this thesis is classifying which of these $\mathcal{N}=(1,0)$ theories could be integrable. Integrability essentially means that a particular system has a very simple dynamical structure underlying it.

Using the AdS/CFT correspondence, we do indeed find that (the bosonic sector) of some of these $6 \mathrm{~d} \mathcal{N}=(1,0)$ theories are integrable in the large- $N$ limit when their quiver structure 
is of a particular form. We find that this is the case for both an infinite constant quiver, and and infinite linear quiver theory. In addition we find that an infinite quiver where all colour groups are proportional to the flavour groups is intergable. This integrable 6d SCFT and its holographic dual are the topic of further investigations in chapter 6 .

Finding an example of an integrable $6 \mathrm{~d}$ SCFT could provide a useful starting point from where we can learn more about the structure of 6d SCFTs, as well as the more general structures needed to describe them properly within the framework of quantum field theory. We can at present only hope that this potentially could have applications describing yet unknown aspects of the real world.

"If you are receptive and humble, mathematics will lead you by the hand"

- Paul Dirac

\subsection{Organisation of this Thesis}

- In chapter 2 we introduce the reader to the Green-Schwarz superstring. We give a very pedagogical introduction to integrability, superstring theory on group manifolds and their cosets, and show explicitly how one can obtain a Lax connection whose flatness condition is equivalent to the equations of motion of the string on various supergravity backgrounds. We also introduce integrable deformations of supergravity backgrounds like (non-)Abelian T-dualities, that deform a the target space of the string theory but preserve integrability on the string worldsheet.

- In chapter 3 we review the AdS/CFT correspondence and its connection to the nearhorizon limit of D-branes in more detail. We study half-BPS $\mathrm{D}_{p}-\mathrm{D}_{p+2}$-NS5 brane set-ups, that give rise to $p$-dimensional half-supersymmetric SCFTs with bifundamental hypermultiplets giving rise to quiver structures for the colour and flavour groups. We then introduce the half-supersymmetric $\mathrm{AdS}_{p+1}$ backgrounds that are thought to be the nearhorizon limit (for $p=3,4,5,6$ ) of these set-ups. We review several studies that have been done on the integrability of strings on these half-supersymmetric $\mathrm{AdS}_{p+1}$ backgrounds, and their relation with (non-)Abelian T-duality. This chapter is largely of a review nature, and partially based on our work in [107].

- In chapter 4 we focus in more detail on the class of half-BPS $\mathrm{AdS}_{7}$ solutions, that are dual to non-Lagrangian $6 \mathrm{~d} \mathcal{N}=(1,0)$ SCFTs. We introduce these $6 \mathrm{~d}$ SCFTs and explain in more detail what makes it difficult to give a conventional Lagrangian description for these theories in section 4.1. We then give a detailed account of their holographic dual descriptions in terms of $\mathrm{AdS}_{7}$ geometries. This chapter is largely of a review nature and partially based on the first half of our work in [136]. 


\section{INTRODUCTION}

- In chapter 5 we study the integrability of string embeddings on these half-BPS $\mathrm{AdS}_{7}$ geometries in detail. We use both analytical methods (based on Kovacic's algorithm), and numerical methods (based on Poincaré sections and Lyapunov exponents). We show that on all (except three) $\mathrm{AdS}_{7}$ backgrounds the (bosonic sector of the) string will be non-integrable. These results are new and based on our work in $[136,106]$. The $\mathrm{AdS}_{7}$ backgrounds on which the (bosonic sector of the) string is integrable are an infinite massless solution, a solution corresponding to an infinite linear quiver (which was not yet emphasized in our earlier publications), and one particular special case that we call $\operatorname{AdS}_{7} \times\left(S^{3}\right)_{\lambda}$.

- In chapter 6, we present a Lax pair for the bosonic section of the string on the $\operatorname{AdS}_{7} \times\left(S^{3}\right)_{\lambda}$ background, as we showed in [106]. We find an interpretation of this geometry as being dual to a $6 \mathrm{~d} \mathcal{N}=(1,0) \mathrm{SCFT}$ with an infinite quiver where the rank of the flavour groups are everywhere proportional to the ranks of the colour groups. These new ideas have been published in [107], and can also be applied to the other half-supersymmetric $\mathrm{AdS}_{p+1}$ backgrounds that we studied earlier in chapter 3 . We suggest that the Type IIA superstring on $\mathrm{AdS}_{7} \times\left(S^{3}\right)_{\lambda}$ corresponds to the large- $N$ limit of the dual $6 \mathrm{~d}$ SCFT, in the limit when the Chern-Simons level of this theory is infinite. We calculate various observables from the holographic dual description.

Besides integrability on AdS backgrounds, we are especially interested in integrable deformations in this thesis. The motivation for this is that the integrable $\mathrm{AdS}_{7} \times\left(S^{3}\right)_{\lambda}$ background can be obtained as the $\lambda$-deformation of $\mathrm{AdS}_{7} \times \mathrm{S}^{3}$ for a particular value of $\lambda$. Integrable deformations allow us to start from a highly symmetric target space on which the (super)string is integrable, and deform it to obtain a different target space that is less symmetric, while the integrability on the string worldsheet will be preserved.

- In chapter 7 we explain the power of integrable deformations (in particular non-Abelian T-duality) as a solution generating technique, and illustrate this by constructing a new integrable supergravity background. We do this by performing a NATD on two $S U(2)$ supspaces of $\mathrm{AdS}_{5} \times S^{5}$. We then study the resulting background in detail. This chapter is largely based on our results published in [82]. 
II. INTEGRABILITY AND SUPERSTRING THEORY

\section{Chapter 2}

\section{Integrability and Superstring Theory}

This chapter serves as a pedagogical introduction to the ideas of classical integrability of superstring theory on group manifolds and coset spaces [13]. We will interrupt this introduction at various points to illustrate some technicalities in more detail, where they are used as tools in the main body of this thesis.

We will first introduce integrability in classical mechanics, field theory, and string theory in section 2.1. We then show how these ideas apply to the bosonic string on a target space that is equivalent to a Lie group or a coset in sections 2.2 and 2.3, where we introduce the principal chiral, and Wess-Zumino-Witten models. Later we generalise this to the GreenSchwarz formalism for the string on a supergroup in section 2.4, 2.5, and 2.6, and explain how integrability is relevant for understanding the dynamics of the superstring on various backgrounds. In section 2.7 we briefly explain the supergravity equations that follow from requiring the beta function on the string worldsheet vanishes. In the last part of this chapter we will introduce in detail (non-)Abelian T-duality and its relation with integrability and integrable deformations of the string target space.

\section{$2.1 \quad$ Integrability}

A dynamical system is called integrable when the number of conserved quantities is equal to the dimensionality of the system. The dynamics of an integrable system is thus maximally constrained, and as a consequence the trajectories of the system in phase space will be (quasi)periodical, and are fixed on a higher dimensional torus in the phase space. 
Let us illustrate this with the simplest example; the $N$-dimensional harmonic oscillator

$$
\mathcal{H}=\frac{1}{2} \sum_{i=1}^{N}\left(p_{i}^{2}+\omega_{i}^{2} x_{i}^{2}\right)
$$

with position variables $x_{i}$ and conjugate momenta $p_{i}$, and equations of motion

$$
\begin{array}{cll}
\dot{p}_{i}=-\frac{\partial \mathcal{H}}{\partial x_{i}}, & \rightarrow & \dot{p}_{i}=-\omega_{i}^{2} x_{i}, \\
\dot{x}_{i}=\frac{\partial \mathcal{H}}{\partial p_{i}}, & \rightarrow & \dot{x}_{i}=p_{i} .
\end{array}
$$

If we plot the trajectories of $\left(x_{i}, p_{i}\right)$ in phase space, we see that for each value of $i$ they trace out circular orbits of constant radius $R^{2}=\frac{1}{2}\left(p_{i}^{2}+\omega_{i}^{2} x_{i}^{2}\right)$. This is because the energy remains fixed along these orbits

$$
\frac{d E}{d t}=\sum_{i=1}^{N}\left(\dot{p}_{i} p_{i}+\omega_{i}^{2} \dot{x}_{i} x_{i}\right)=0 .
$$

Since this is the case for all values of $i$, the $N$-dimensional oscillator traces out an $N$-dimensional torus in its $2 N$-dimensional phase space. Let us for a moment concentrate on the trajectories in the four-dimensional space spanned by $\left(x_{i}, x_{j}, p_{i}, p_{j}\right)$, which will be confined to the surface of a two-dimensional torus. When the ratio between the different frequencies $\omega_{i}$ and $\omega_{j}$ is a rational number, the orbit will close on itself after winding a certain number of times around the torus. We then say the motion is periodic. It is of course also possible that the ratio between the frequencies is not a rational number (for example $\omega_{i}=1$ and $\omega_{2}=\sqrt{2}$ ). When this is the case, we say the two frequencies are incommensurable and the trajectory will trace out the surface of the torus in phase space without coming back to itself. This is called quasi-periodic motion.

Peter Lax showed [1] that a dynamical system is integrable when there exists a pair of time-independent Hermitian differential operators $L$ and $M$, such that the equations of motion of the system can be written as

$$
\partial_{t} L=[M, L]
$$

Finding this Lax pair, or proving it does not exist, for a generic system is a non-trivial problem. Once a Lax pair has been found one can immediately obtain the conserved quantities $Q_{n}$ for the system as

$$
Q_{n}=\operatorname{Tr}\left[L^{n}\right]
$$

Using the definition for the Lax in eq.(2.3) one can see, using the cyclicity of the trace, that these quantities are indeed conserved as

$$
\frac{d Q_{n}}{d t}=\sum_{i=0}^{n-1} \operatorname{Tr}\left[L^{i}[M, L] L^{n-1-i}\right]=n \operatorname{Tr}\left[L^{n-1} M L-L^{1} M\right]=0
$$

For a more detailed review, see [2]. 


\section{INTEGRABILITY AND SUPERSTRING THEORY}

For the $N$-dimensional harmonic oscillator this is indeed the case, and the corresponding Lax pair is given by

$$
L=\left(\begin{array}{ccc}
L_{1} & & \\
& \ddots & \\
& & L_{N}
\end{array}\right), \quad M=\left(\begin{array}{ccc}
M_{1} & & \\
& \ddots & \\
& & M_{N}
\end{array}\right),
$$

where

$$
L_{i}=\left(\begin{array}{cc}
p_{i} & \omega_{i} x_{i} \\
\omega_{i} x_{i} & -p_{i}
\end{array}\right), \quad M_{i}=\frac{\omega_{i}}{2}\left(\begin{array}{cc}
0 & -1 \\
1 & 0
\end{array}\right) .
$$

The reader can verify that $\operatorname{Tr}\left[L^{n}\right]$ gives either 0 (when $n$ is odd), or is proportional to the conserved energy $\sum_{i=1}^{N} E_{i}=\mathcal{H}$ (when $n$ is even).

\subsubsection{Integrable Field Theories}

A field theory has an infinite number of position and momentum variables, one for every point in space, which makes it at first less obvious how to generalise the notion of conserved charges, as their number will now be infinite.

We can however still generalise the notion of a Lax pair to apply to field theories. Since the system now depends on both time and position, there has to be a pair $L$ and $M$, in terms of which one can write the equations of motion as

$$
\frac{\partial L}{\partial t}-\frac{\partial M}{\partial x}=[M, L]
$$

Note this is identical to the flatness condition for a connection, known as a Lax connection

$$
F_{a b}=\partial_{a} L_{b}-\partial_{b} L_{a}-\left[L_{a}, L_{b}\right]=0,
$$

where the different spatial and temporal components of the Lax connection $L_{a}=\left(L_{t}, L_{x}\right)$ are now equal to the Lax pair $(L, M)$. A field theory is thus classically integrable when it it can, with some change of variables, be rewritten as a pure-gauge theory. A well-known example of this is the electromagnetic vacuum, which is described by a $U(1)$ vector field $A_{\mu}$ with vanishing field strength. Similar to the case of the harmonic oscillator, the solutions are non-interacting harmonic modes of the form $\Phi(t)=A e^{i \omega t}$. We thus quickly see the analogy with the harmonic oscillator: for an integrable field theory the dynamics of the field is linear, harmonic and (quasi-)periodic. It is surprising that many complicated looking field theories can with a clever redefinition of variables, be rewritten as a simple pure-gauge theory.

For more complicated dynamical systems (with more complicated equations of motion) we will see that we can introduce an additional complex parameter $z$ - known as the spectral parameter - so that the flatness condition (2.9) for the Lax connection $L_{a}(z)$ is satisfied for all 
values of $z$. In chapter 2 we will show various examples of systems where the flatness condition can be expanded into different powers of $z$, such that each of the different powers of $z$ comes with one of the different equations of motion for the system.

Analogous to how we earlier showed there is a number of (non)-local conserved charges $Q_{n}$, equal to the dimension of the system, one can define an infinite number of conserved charges for an integrable field theory. To find these we first consider the parallel transport of the spatial component $L_{x}$ of the Lax connection along a line stretching from $x_{-}$to $x_{+}$

$$
T(z)=\mathcal{P} \exp \left(\int_{x_{-}}^{x_{+}} d x L_{x}(z)\right)
$$

where the points $x_{-}$and $x_{+}$have to be identified by compactifying the spatial direction, and $\mathcal{P}$ indicates the path-ordered exponential. Analogous to eq.(2.3) one can now show that

$$
\partial_{t} T(z)=\left[L_{t}(z), T(z)\right]
$$

To see the analogy with eq.(2.3 one has to remember that the temporal component of the Lax connection $L_{t}(z)$ is equal to the $M(z)$ in eq.(2.3). From the monodromy of the Lax connection one can then define the transfer matrix $t(z)$, from which one can obtain an infinite set of conserved charges $Q_{n}$ as the different terms in the Taylor expansion of the spectral parameter

$$
t(z)=\operatorname{Tr}[T(z)]=\sum_{n=0}^{\infty} Q_{n} z^{n} .
$$

\subsubsection{Integrability in String Theory}

Strings are 1-dimensional objects on a $D$-dimensional target space, and trace out a 2 -dimensional worldsheet $\Sigma$ as they move in time. Their dynamics (ignoring fermionic field on the worldsheet for the moment) is such that they minimise the area traced out by their worldsheet, and are described by the Nambu-Goto action

$$
S_{N G}\left[X^{\mu}\right]=-T_{s} \int_{\Sigma} d^{2} z \sqrt{-\operatorname{det} \partial_{a} X^{\mu} \partial_{b} X_{\mu}}
$$

Here the $X^{\mu}$ are the coordinates of the target space, with $\mu=0, \ldots, D$. The Latin indices $a, b=$ 0,1 range over the two dimensional worldsheet coordinates $z^{a}$. These strings acts analogous to rubber bands that want to minimise their size in order to minimise their potential energy. The coupling $T=\left(2 \pi \alpha^{\prime}\right)^{-1}$ in front is also known as the string tension. The smaller the tension $T_{s}$, the more wildly the strings can fluctuate with their size characterised by the string length $\ell_{s}=\sqrt{\alpha^{\prime}}$.

Since the quantization of the Nambu-Goto action is rather difficult, it is common to promote the pull-back of the worldsheet metric $g^{a b}=\partial_{a} X^{\mu} \partial_{b} X_{\mu}$ to be an independent field and rewrite 


\section{INTEGRABILITY AND SUPERSTRING THEORY}

the action as the Polyakov action

$$
S_{P}\left[X^{\mu}, g^{a b}\right]=\frac{-1}{4 \pi \alpha^{\prime}} \int_{\Sigma} d^{2} z \sqrt{-g} g^{a b} \partial_{a} X^{\mu} \partial_{b} X^{\nu}
$$

which can be seen to be classically equivalent to eq.(2.13) by using its equations of motion to eliminate the worldsheet metric $g^{a b}$. The Polyakov action is invariant under both worldsheet diffeomorphisms $z^{a} \rightarrow f\left(z^{a}\right)$, and local Weyl rescalings of the metric $g_{a b} \rightarrow e^{2 \omega(z)} g_{a b}$. Here - as in the rest of this thesis - we will gauge-fix the worldsheet metric to be the flat metric $\eta^{a b}$ and work in lightcone coordinates on the worldsheet $z^{ \pm}=\frac{1}{\sqrt{2}}(\tau \pm \sigma)$, where the Polyakov action (on a flat target space) takes the form

$$
S=\frac{1}{2 \pi \alpha^{\prime}} \int_{\Sigma} d^{2} z \partial_{a} X^{\mu} \partial^{a} X_{\mu}
$$

When we now consider the Polyakov action for the string on an arbitrary background spacetime with metric $G_{\mu \nu}(X)$ and an possible additional antisymmetric tensor field $B_{\mu \nu}(X)$ it will be of the form

$$
S_{P}=\frac{1}{2 \pi \alpha^{\prime}} \int_{\Sigma} d^{2} z\left(\eta^{a b} G_{\mu \nu}(X)+\epsilon^{a b} B_{\mu \nu}(X)\right) \partial_{a} X^{\mu} \partial_{b} X^{\nu}
$$

This action is also known as the two-dimensional non-linear sigma model, and the equations of motion for for this action are - as the name implies - in general non-linear.

When we take the path integral over the action in eq.(2.16) we have to sum over all possible worldsheets, including ones where multiple strings split or join together. To suppress the splitting and joining of strings we can add a term $S_{\text {int }}$ to the action $S_{\text {string }}=S_{P}+S_{\text {int }}$, that weights each string worldsheet in the path integral with its Euler number $\chi$

$$
S_{\text {int }}=\Phi(X) \chi, \quad \chi=\frac{1}{4 \pi} \int_{\Sigma} d^{2} z R(z) .
$$

Here we used that for a two dimensional worldsheet the Euler number can be directly expressed in terms of the integral over the Ricci scalar $R(z)$ of the string worldsheet. The dilaton on the target space $\Phi(z)$ acts as a the string coupling $g_{s}=e^{\Phi}(X)$, and suppresses the amplitudes that involve the splitting and joining of strings with a factor $g_{s}^{-2}$.

We thus have two parameters in string theory, the string tension $T=\left(2 \pi \alpha^{\prime}\right)^{-1}$ (or string length $\left.\ell_{s}=\sqrt{\alpha^{\prime}}\right)$ and the string coupling $g_{s}=e^{\Phi}(X)$. The limit where we can solve the action for the string exactly is when both $g_{s} \rightarrow 0$ (where we can ignore the string interactions and consider a free string theory), and when the target spacetime only varies on scales $R \gg \ell_{s}$.

In this thesis we will be especially interested in target spaces that have a large number of isometries and can be thought of as Lie groups or their cosets. The worldsheet action of the string then becomes a classically integrable field theory. This allows us to write the equations of motion in Lax form, allowing exact solutions. We will introduce these ideas in more detail in the next sections of this chapter. 


\section{$2.2 \quad$ Principal Chiral Model}

A Lie group is a finite dimensional smooth manifold with a group structure. It is therefore easer to think of more symmetric target spaces for the string as Lie groups. In this section we will introduce the Polyakov action (2.16) on a group manifold, and demonstrate how the equations of motion for the string are integrable and admit a Lax form in these cases an example. We show this explicitly for the group $S U(2)$, and then introduce symmetric spaces that can be written as cosets of Lie groups.

We start from the action for the sigma model of eq.(2.16), and assume we have a target space with a vanishing $B$-field for a moment

$$
S=\frac{k}{2 \pi} \int_{\Sigma} d^{2} z \eta^{a b} \partial_{a} X^{\mu} \partial_{b} X^{\nu} G_{\mu \nu}
$$

If the target space is a (non-Abelian) Lie group with group elements $g=e^{X^{A} T_{A}}$, and generators $T_{A}$, we can rewrite this action as

$$
\begin{aligned}
\partial_{a} X^{\mu} \partial^{a} X^{\nu} G_{\mu \nu} & =\partial_{a} X^{A} \partial^{a} X^{B} \operatorname{Tr}\left[T_{A} T_{B}\right]=\operatorname{Tr}\left[g^{-1} \partial_{a} X^{A} T_{A} g g^{-1} \partial^{a} X^{B} T_{B} g\right] \\
& =\operatorname{Tr}\left[g^{-1} \partial_{a} g g^{-1} \partial^{a} g^{-1}\right]=\operatorname{Tr}\left[\partial_{a} g \partial^{a} g^{-1}\right]
\end{aligned}
$$

where the $g$ and $g^{-1}$ cancel due to the cyclicity of the trace. Here $G_{A B}=\left\langle T_{A} T_{B}\right\rangle=\operatorname{Tr}\left[T_{A} T_{B}\right]$ is the Killing form on the group, and we used that $\partial g^{-1}=-g^{-1} \partial g g^{-1}$. We can write this action either in terms of the left-invariant or right-invariant currents, that are invariant under the multiplication of $g$ with an arbitrary group element from the left or right respectively.

$$
\begin{array}{rll}
\text { left-invariant current: } & J_{a}^{A}=g^{-1} \partial_{a} g & \text { invariant under } g \rightarrow g_{0} g \\
\text { right-invariant current: } & \tilde{J}_{a}^{A}=\partial_{a} g g^{-1} & \text { invariant under } g \rightarrow g g_{0}
\end{array}
$$

Using the cyclicity of the trace this allows us to write the action as

$$
\operatorname{Tr}\left[\partial_{a} g \partial^{a} g^{-1}\right]=\operatorname{Tr}\left[J_{a} J^{a}\right]=\operatorname{Tr}\left[\tilde{J}_{a} \tilde{J}^{a}\right]
$$

Notice the action is invariant under both a global multiplication of $g$ with an arbitrary group element $g_{0}$ acting from either the left or right, and there is thus a global $G_{L} \times G_{R}$ symmetry. Because of this, the action is also known as the Principal Chiral Model (PCM).

Varying the action gives us the equations of motion that are equivalent to the conservation 


\section{INTEGRABILITY AND SUPERSTRING THEORY}

of the left (or right)-invariant current

$$
\begin{array}{rlrl}
\delta \mathcal{L} \sim & 2 \operatorname{Tr}\left[\delta g^{-1} \partial_{a} g g^{-1} \partial^{a} g+\partial_{a} \delta g g^{-1} \partial^{a} g g^{-1}\right] \\
& =-2 \operatorname{Tr}\left[\delta g g^{-1}\left(\partial_{a} g\right) g^{-1}\left(\partial^{a} g\right) g^{-1}+\delta g \partial_{a}\left(g^{-1} \partial^{a} g g^{-1}\right)\right] \\
& =+2 \operatorname{Tr}\left[\delta g g^{-1}\left(\partial_{a} g\right) g^{-1}\left(\partial^{a} g\right) g^{-1}-\delta g g^{-1} \partial_{a} \partial^{a} g g^{-1}\right] \\
& =-2 \operatorname{Tr}\left[\delta g\left(\partial_{a} g^{-1} \partial^{a} g+g^{-1} \partial_{a} \partial^{a} g\right) g^{-1}\right] \\
& =-2 \operatorname{Tr}\left[\Delta \partial\left(g^{-1} \partial g\right)\right], & \rightarrow & \partial_{a} J^{a}=0 \\
& =+2 \operatorname{Tr}\left[\Delta \partial\left(\partial g^{-1} g\right)\right], & \rightarrow & \partial_{a} \tilde{J}^{a}=0 .
\end{array}
$$

Here we used that $\delta g^{-1}=-g^{-1} \delta g g^{-1}$, and we defined $\Delta=g^{-1} \delta g$.

It is more convenient to just work with the currents $J_{a}$ or $\tilde{J}_{a}$ as the fundamental field, instead of the group elements $g$. It is convention to work with the left-invariant current $J_{a}$ - also called the Maurer-Cartan form on the group - which we will do from here on. To obtain the equations of motion from the action (2.20) in terms of $J_{a}$, we consider the variation of $J_{a}$ under the infinitesimal variation $\delta g=g \epsilon$, with $\epsilon$ an arbitrary group element.

$$
\begin{aligned}
\delta J_{a} & =\delta g^{-1} \partial_{a} g+g^{-1} \partial_{a} \delta g=-\epsilon g^{-1} \partial_{a} g+g^{-1} \partial_{a} g \epsilon+\partial_{a} \epsilon \\
& =\partial_{a} \epsilon+\left[J_{a}, \epsilon\right]
\end{aligned}
$$

When we insert this variation for $\delta J_{a}$ in the action, the commutator will drop out due to the cyclicity of the trace. After partial integration we obtain

$$
\delta \mathcal{L}=2 \operatorname{Tr}\left[J^{a} \delta J_{a}\right]=-2 \operatorname{Tr}\left[\epsilon \partial_{a} J^{a}\right], \quad \rightarrow \quad \partial_{a} J^{a}=0
$$

Which is equivalent to the equation of motion we obtained earlier in eq.(2.21).

In addition to the equations of motion, the current $J_{a}$ has the form of a pure-gauge potentential and has by construction a vanishing curvature. Note this is an identity that holds by construction and is not an equation of motion.

$$
\begin{aligned}
F_{a b}= & \partial_{a} J_{b}-\partial_{b} J_{a}+\left[J_{a}, J_{b}\right] \\
= & \left(\partial_{a} g^{-1}\right)\left(\partial_{b} g\right)-\left(\partial_{b} g^{-1}\right)\left(\partial_{a} g\right)+g^{-1} \partial_{a} \partial_{b} g-g^{-1} \partial_{b} \partial_{a} g+ \\
& +g^{-1}\left(\partial_{a} g\right) g^{-1}\left(\partial_{b} g\right)-g^{-1}\left(\partial_{b} g\right) g^{-1}\left(\partial_{a} g\right) \\
= & 0
\end{aligned}
$$

\section{An Example: SU(2)}

As an example we will here briefly consider the group $S U(2) \cong S^{3}$, generated by the Pauli matrices, normalised as

$$
T_{A}=\frac{1}{\sqrt{2}}\left\{\left(\begin{array}{cc}
0 & 1 \\
1 & 0
\end{array}\right), \quad\left(\begin{array}{cc}
0 & -i \\
i & 0
\end{array}\right), \quad\left(\begin{array}{cc}
1 & 0 \\
0 & -1
\end{array}\right)\right\}
$$


with commutation relations $\left[T_{A}, T_{B}\right]=i \sqrt{2} \epsilon^{A B C} T_{C}$. The Killing form is then given by

$$
G_{A B}=\left\langle T_{A} T_{B}\right\rangle=\operatorname{Tr}\left[T_{A} T_{B}\right]=\delta_{A B}
$$

We can obtain the group elements by exponentiating the generators

$$
g=e^{i \alpha \overrightarrow{\mathbf{n}}^{A} T_{A}}=\left(\begin{array}{cc}
\cos \alpha+i \cos \beta \sin \alpha & e^{-i \gamma} \sin \alpha \sin \beta \\
-e^{i \gamma} \sin \alpha \sin \beta & \cos \alpha-i \cos \beta \sin \alpha
\end{array}\right)
$$

where we used the parametrisation $\overrightarrow{\mathbf{n}}^{A}=\{-\sin \beta \sin \gamma, \sin \beta \cos \gamma, \cos \beta\}$.

When we have a field that takes value on a Lie group, its value on the group differs for different points in space-time $g(\vec{x}, t)$, so that we can take derivatives of the group element as

$$
\partial_{a} g=e^{i \alpha \overrightarrow{\mathbf{n}}^{A} T_{A}}=e^{i \alpha \overrightarrow{\mathbf{n}}^{A} T_{A}} i\left(\partial_{a} \alpha \overrightarrow{\mathbf{n}}^{A}\right) T_{A}
$$

Giving us for the left invariant current

$$
J_{a}=g^{-1} \partial_{a} g=i\left(\partial_{a} \alpha \overrightarrow{\mathbf{n}}^{A}\right) T_{A} .
$$

We can now decompose the matrix $J_{a}=J_{a}^{A} T_{A}$ into its components on the different generators $T_{A}$. We can then write

$$
=i\left(\begin{array}{c}
-\sin \gamma(\sin \beta \mathrm{d} \alpha+\alpha \cos \beta \mathrm{d} \beta)-\alpha \sin \beta \cos \gamma \mathrm{d} \gamma \\
\cos \gamma(\sin \beta \mathrm{d} \alpha+\alpha \cos \beta \mathrm{d} \beta)-\alpha \sin \beta \sin \gamma \mathrm{d} \gamma \\
\cos \beta \mathrm{d} \alpha-\alpha \sin \gamma \mathrm{d} \gamma
\end{array}\right) \cdot\left(\begin{array}{c}
T_{1} \\
T_{2} \\
T_{3}
\end{array}\right)
$$

where with $\mathrm{d} \alpha, \mathrm{d} \beta$, and $\mathrm{d} \gamma$ we mean the shorthand notation $\mathrm{d} f=\partial_{a} X^{f}$ for the coordinates we introduced to parametrise the group. If we now work out $\operatorname{Tr}\left[J_{a} J^{a}\right]$, and use eq.(2.26) for the Killing form $\operatorname{Tr}\left[T_{A} T_{B}\right]=\delta_{A B}$, we see that the sigma model on $\mathrm{SU}(2)$ looks exactly like the Polyakov action on the three-sphere.

$$
\operatorname{Tr}\left[J_{a}^{A} J^{A a}\right]=(\sin \beta \mathrm{d} \alpha+\alpha \cos \beta \mathrm{d} \beta)^{2}+(\cos \beta \mathrm{d} \alpha-\alpha \sin \gamma \mathrm{d} \gamma)^{2}+\alpha^{2} \sin ^{2} \beta \mathrm{d} \gamma^{2},
$$

where we used the shorthand notation $\mathrm{d} f^{2}=\partial_{a} X^{f} \partial_{b} X^{f}$.

The relation between the group manifold written in terms of of generators $g=e^{i \alpha \overrightarrow{\mathbf{n}}}$, and in terms of coordinates can be understood more easily be realising that one can identify the Maurer-Cartan form $J_{a}=g^{-1} \partial_{a} g=J_{a}^{A} T_{A}$ with the vielbeins on the group manifold $J_{a}^{A}=$ $\partial_{a} X^{\mu} e_{\mu}^{A}$. In terms of the vielbein we can write the action of eq.(2.30) as

$$
\operatorname{Tr}\left[J_{a} J^{a}\right]=J_{a}^{A} J_{A}^{a}=\eta^{a b} e_{\mu}^{A} \partial_{a} X^{\mu} e_{\nu}^{B} \partial_{b} X^{\nu} G_{A B}=\eta^{a b} \partial_{a} X^{\mu} \partial_{b} X^{\nu} G_{\mu \nu}
$$

which gives us the non-linear sigma model on a target space expressed in terms of its coordinates and metric $G_{\mu \nu}$. 


\section{INTEGRABILITY AND SUPERSTRING THEORY}

\subsubsection{Coset Sigma Model}

We will next consider the case where the group is a coset $G / H$. We identify the points $g \sim g h$ that are related by an element $h \in H$ acting from the right. This means both the action and the equations of motion should be invariant under this multiplication with $h \in H$, so that the entire orbit of an element $g$ under the action of $H$ is seen as a single equivalence class.

To this end we first split $J$ into a part $J^{(0)}$ that is valued on $\mathfrak{h}$, and $J^{(1)}$ valued on the algebra $\mathfrak{p}$ of the remaining coset $G / H$.

$$
J=J^{(0)} \oplus J^{(1)} .
$$

Under $g \rightarrow g h$ the Maurer-Cartan form transforms as $J_{a} \rightarrow h^{-1} J_{a} h+h^{-1} \partial_{a} h$. The additional component $h^{-1} d h$ is valued on $\mathfrak{h}$, and can thus only come from $J^{(0)}$. The two components of the Maurer-Cartan form thus transform as

$$
\begin{aligned}
& J^{(0)}=h^{-1} J^{(0)} h+h^{-1} d h \\
& J^{(1)}=h^{-1} J^{(1)} h
\end{aligned}
$$

To obtain the action for the PCM on the coset we can gauge out the subgroup $H$ from the action on the total group $G$, by introducing a gauge field $A_{a}$ that is valued only on $\mathfrak{h}$

$$
\begin{aligned}
S & =\frac{k}{2 \pi} \int_{\Sigma} d^{2} z \operatorname{Tr}\left[\left(J_{a}-A_{a}\right)\left(J^{a}-A^{a}\right)\right], \\
& =\frac{k}{2 \pi} \int_{\Sigma} d^{2} z \operatorname{Tr}\left[\left(J_{a}^{0)}-A_{a}\right)\left(J^{(0) a}-A^{a}\right)-J_{a}^{(1)} J^{(1) a}\right] .
\end{aligned}
$$

When $H$ is a Lie-subgroup, $\mathfrak{h}$ and $\mathfrak{p}$ are orthogonal under the Killing form, that is $\operatorname{Tr}\left[J^{(0)} J^{(1)}\right]=$ 0 . The cross terms between $J^{(0)}$ and $J^{(1)}$ therefore vanish in the trace of the action above. If we vary with respect to this gauge field $A_{a}$ we find the equation of motion

$$
\delta S=2 \operatorname{Tr}\left[\delta A_{a}\left(J^{(0) a}-A^{a}\right)\right], \quad \rightarrow \quad J_{a}^{(0)}=A_{a}
$$

Alternatively, we could have integrated out $\left(J_{a}^{(0)}-A_{a}\right)$, which is trivial as it only appears quadratically. In both cases we find the effective action for the remaining group elements on $G / H$ to be of the form

$$
S=\frac{k}{2 \pi} \int_{\Sigma} d^{2} z \operatorname{Tr}\left[J^{(1) a} J_{a}^{(1)}\right]
$$

When we now obtain the equations of motion we again vary $J_{a}$ by an arbitrary group element as we did in eq.(2.22). The variation of $J_{a}$ can now be split in a part on $\mathfrak{h}$ and a part on $\mathfrak{p}$.

$$
\delta J_{a}=\partial_{a} \epsilon+\left[J_{a}, \epsilon\right]=\partial_{a} \epsilon+\left[J_{a}^{(0)}, \epsilon\right]+\left[J_{a}^{(1)}, \epsilon\right]=D_{a} \epsilon+\left[J_{a}^{(1)}, \epsilon\right]
$$


where $D_{a}=\partial_{a}+\left[J^{(0)}, \cdot\right]$. Note the cyclicity of the trace now only causes the $\left[J^{(1)}, \epsilon\right]$ commutator to vanish. This gives us for the variation of the action and the equations of motion

$$
\delta \mathcal{L}=2 \operatorname{Tr}\left[\left(D_{a} \epsilon+\left[J_{a}^{(1)}, \epsilon\right]-A_{a}\right)\left(J^{a}-A^{a}\right)\right]=2 \operatorname{Tr}\left[\epsilon D_{a} J^{(1) a}\right], \quad \rightarrow \quad D_{a} J^{(1) a}=0
$$

where we inserted the equation of motion $A_{a}=J_{a}^{(0)}$. The current on the subgroup $J^{(0)}$ now appears in the covariant derivative, so that the equations of motion transform in the adjoint under $H$.

Let us now have another look at the flatness condition. By construction the Maurer-Cartan form on the total group $G$ is still flat, as we showed in eq.(2.24). If we now decompose $J$ into its $\mathfrak{h}$ and $\mathfrak{p}$-valued components we can write the flatness condition on these components as

$$
\begin{aligned}
F_{a b} & =\partial_{a} J_{b}-\partial_{b} J_{a}+\left[J_{a}, J_{b}\right] \\
& =F_{a b}^{(0)}+D_{a} J_{b}^{(0)}-D_{b} J_{a}^{(0)}+\left[J_{a}^{(1)}, J_{b}^{(1)}\right]=0
\end{aligned}
$$

Where $F_{a b}^{(0)}$ indicates the curvature of $J^{(0)}$. Since the equations of motion fixed $D_{a} J^{(1) a}=0$, the flatness condition can be rewritten as

$$
\partial_{a} J_{b}^{(0}-\partial_{b} J_{a}^{(0}+\left[J^{(0)}, J^{(0}\right]+\left[J^{(1)}, J^{(1)}\right]=0
$$

The reader can check again that this equation now also transforms in the adjoint of $h$. This gives us (in light cone coordinates) the three independent equations for the Polyakov action

$$
\begin{aligned}
D_{+} J_{-}^{(1)} & =0 \\
D_{-} J_{+}^{(1)} & =0 \\
\partial_{+} J_{-}^{(0)}-\partial_{-} J_{+}^{(0)}+\left[J_{+}^{(0)}, J_{-}^{(0)}\right]+\left[J_{+}^{(1)}, J_{-}^{(1)}\right] & =0
\end{aligned}
$$

with $D_{a} \cdot=\left[\partial_{a}+J_{a}^{(0)}, \cdot\right]$

\subsubsection{Symmetric Cosets and $\mathbb{Z}_{2}$ grading}

Let's briefly check the commutators of the elements $H \in \mathfrak{h}$ and $P \in \mathfrak{p}$. For the above construction it is in general irrelevant how $H$ and $P$ commute. Since $H$ is the Maurer-Cartan form on a subalgebra

$$
[H, H] \subset H
$$

and the structure constants $f_{h h p}=0$. Since the structure constants are anti-symmetric we know that $f_{h p h}=-f_{h p h}=0$. This tells us the commutator of $H$ and $P$ has to be of the from

$$
[H, P] \subset P
$$




\section{INTEGRABILITY AND SUPERSTRING THEORY}

At last the commutator of $H$ and $P$ can - on a general coset - bring us to either $H$ or $P$.

In the previous section we were considering the case where

$$
[P, P] \subset H
$$

This is a special kind of coset, also known as a symmetric coset. The algebra then has an additional structure known as a grading. We can associate a value of 0 with the elements $H$, and a value of 1 with the elements of $P$, we find that if the commutators add these values (modulo 2) the commutators of a symmetric coset preserve this graded structure:

$$
\begin{array}{ccc}
{[\mathfrak{h}, \mathfrak{h}] \subset \mathfrak{h},} & {[\mathfrak{h}, \mathfrak{p}] \subset \mathfrak{p},} & {[\mathfrak{p}, \mathfrak{p}] \subset \mathfrak{h}} \\
(0+0)=0 \bmod 2=0, & (0+1)=1 \bmod 2=1, & (1+1)=2 \bmod 2=0
\end{array}
$$

This grading is the reason we introduced the notation $J^{(0)}$ valued on $\mathfrak{g}^{(0)}=\mathfrak{h}$ and $J^{(1)}$ valued on $\mathfrak{g}^{(1)}=\mathfrak{p}$ in the previous section. When there is such a grading on the algebra, it is possible to define an involutive (or projective) automorphism $\Omega$ on the algebra; an operator $\Omega$ for which $\Omega^{2}=\mathbb{I}$ that preserves the structure of the algebra

$$
\Omega([X, Y])=[\Omega(X), \Omega(Y)]
$$

The generators of $H$ and $P$ must have eigenvalues of \pm 1 under $\Omega$, s we can choose for example

$$
\Omega(\mathfrak{h})=\mathfrak{h}, \quad \Omega(\mathfrak{p})=-\mathfrak{p} .
$$

We can now use this automorphism to separate the generators of $H$ and $P$. This an be done by decomposing the algebra $\mathfrak{g}=\mathfrak{h} \oplus \mathfrak{p}=\mathfrak{g}^{(0)} \oplus \mathfrak{g}^{(1)}$ into the direct sum of two differently graded eigenspaces of $\Omega$

$$
\mathfrak{g}^{(n)}=\frac{1}{2}\left(\mathfrak{g}+(-1)^{n} \Omega^{n}(\mathfrak{g})\right)
$$

which transform under $\Omega$ as

$$
\Omega\left(\mathfrak{g}^{(n)}\right)=(-1)^{n} \mathfrak{g}^{(n)}
$$

As a result it is then possible to decompose the algebra-valued Maurer-Cartan form $J$ according to this grading $J=J^{(0)} \oplus J^{(1)}$ so that the different currents satisfy the same commutation relations as in eq. $(2.45)$

$$
\left[J^{(i)}, J^{(j)}\right] \subset J^{(i+j)}, \quad \text { i, } j \bmod \mathbb{Z}_{2}
$$

When we consider the sigma model on such a space, notice the equations of motion and the flatness condition we obtained earlier in eq.(2.41) are now also organised according to the 
different gradings

$$
\begin{aligned}
D_{+} J_{-}^{(1)} & =0, & & \text { graded 0 } \\
D_{-} J^{(1)} & =0, & & \text { graded 0 } \\
\partial_{+} J_{-}^{(0)}-\partial_{-} J_{+}^{(0)}+\left[J_{+}^{(0)}, J_{-}^{(0)}\right]+\left[J_{+}^{(1)}, J_{-}^{(1)}\right] & =0, & & \text { graded 1 }
\end{aligned}
$$

As a consequence it is easy to write down a Lax connection, the flatness condition of which reproduces the above equations of motion

$$
L_{ \pm}=J_{ \pm}^{(0)}+z^{ \pm 2} J_{ \pm}^{(1)}
$$

where $z$ is the spectral parameter.

\section{Lie Groups and Cosets as Symmetric Spaces}

Note that every Lie group itself is also a symmetric space. This can easily be seen as we can construct every Lie group as a coset of the form

$$
G \cong \frac{G_{L} \times G_{R}}{G}
$$

To see the graded commutation relations of eq.(2.45) appear explicitly, we define an automorphism that acts on the algebra as

$$
\Omega\left(\mathfrak{g}_{L}\right)=\mathfrak{g}_{R}, \quad \Omega\left(\mathfrak{g}_{R}\right)=\mathfrak{g}_{L}
$$

where $\mathfrak{g}_{L}$ and $\mathfrak{g}_{R}$ are the algbras of $G_{L}$ and $G_{R}$ in the top line of eq.(2.45). We then find this gives us for the eigenspaces

$$
\mathfrak{h}=\mathfrak{g}_{L}+\mathfrak{g}_{R}, \quad \mathfrak{p}=\mathfrak{g}_{L}-\mathfrak{g}_{R},
$$

one can check that under this identification the commutators indeed obtain the structure of eq.(2.52), and one can consequently write a Lax of the form in eq.(2.51) for the sigma model on any Lie group.

The reason these cosets are called symmetric is that - when we see them as manifolds - they are equivalent to symmetric spaces, where there is a maximal number of Killing vectors. Meaning we can move or rotate the manifold along any of its directions and it will remain identical. Examples of such manifolds are flat Minkowski or Euclidean space, positively curved spherical or de Sitter space, as well as negatively curved hyperbolic and anti-de Sitter space.

$$
\begin{aligned}
\mathrm{S}^{n} \cong \frac{S O(n+1)}{S O(n)}, & \mathbb{R}^{n} \cong \frac{I S O(n)}{S O(n)} & \mathrm{H}^{n} \cong \frac{S O(1, n)}{S O(n)} \\
\mathrm{d} S^{n} \cong \frac{S O(1, n)}{S O(1, n-1)} & \mathbb{M}^{n} \cong \frac{I S O(1, n)}{S O(1, n)} & \mathrm{AdS}^{n} \cong \frac{S O(2, n-1)}{S O(1, n-1)}
\end{aligned}
$$




\section{INTEGRABILITY AND SUPERSTRING THEORY}

Let us briefly consider the above identities in more detail. The group $S O(3)$ can be though of as the group of all special orthogonal rotations acting on a vector in three-dimensional space. When we trace out the surface that is spanned by $S O(3)$ acting on a vector it spans a two sphere. However, $S^{2}$ is actually smaller than the group $S O(3)$, as vectors that are rotated around their axis will all be mapped to the same point on the sphere. To obtain $S^{2}$ we have to gauge out the rotation group $S O(2)$, so that $S^{2} \cong S O(3) / S O(2)$. Alternatively we can see the original group as a manifold of the form $S O(3) \cong S^{2} \times_{w} S^{1}$, where $\times_{w}$ indicates a warped product.

\subsection{Wess-Zumino-Witten Model}

When we consider the beta function of the PCM, it will not vanish for a generic Lie group. The vanishing of the beta function is however required if we want to identify the non-linear sigma model with the Polyakov action for a string in eq.(2.16). This vanishing of the beta function can be achieved by including the coupling to an anti-symmetric $B_{\mu \nu}$-field on the target space with field strength $H_{3}=\mathrm{d} B_{2}$. This $B_{\mu \nu}$ field is sometimes called a torsion term as we can think of it as containing the torsion (anti-symmetric) terms of a more generalised metric $D_{\mu \nu}=G_{\mu \nu}+B_{\mu \nu}$. In the physics literature it is convention to define the metric $G_{\mu \nu}$ to contain symmetric terms only, and consider the torsion of $D_{\mu \nu}$ to be an independent field. We will use this notation throughout this thesis.

If we want to adjust the PCM action in order to contain such anti-symmetric terms on the target space, a first guess could be

$$
S=S_{P C M}+\frac{k}{2 \pi} \int_{\Sigma} d^{2} z \epsilon^{a b} \operatorname{Tr}\left[J_{a} J_{b}\right]
$$

however, due to the cyclicity of the trace such an additional term would vanish.

In order to construct a group valued action that does give rise to such a torsion term, Witten suggested to add a Wess-Zumino (WZ) term to the PCM action [14]. The resulting action is known as the Wess-Zumino-Witten (WZW) model and has an action that in terms of the group elements $g$ is of the form

$$
\begin{aligned}
S_{W Z W} & =S_{P C M}+S_{W Z} \\
& =\frac{k}{2 \pi} \int_{\Sigma_{2}} d^{2} z \operatorname{Tr}\left[\partial_{a} g \partial^{a} g^{-1}\right]-\frac{k}{6 \pi} \int_{\mathcal{M}_{3}} d^{3} z \epsilon^{a b c} \operatorname{Tr}\left[\left(g^{-1} \partial_{a} g\right)\left(g^{-1} \partial_{b} g\right)\left(g^{-1} \partial_{c} g\right)\right],
\end{aligned}
$$

or when written in terms of the Maurer-Cartan form

$$
S_{W Z W}=\frac{k}{2 \pi} \int_{\Sigma_{2}} \operatorname{Tr}[J J]-\frac{k}{6 \pi} \int_{\mathcal{M}_{3}} \operatorname{Tr}[J \wedge J \wedge J] .
$$

Note that this additional Wess-Zumino term does not spoil the global $G_{R} \times G_{L}$ symmetry we introduced in section 2.2, and that it furthermore involves an integral over a three-dimensional 
space $\mathcal{M}_{3}$. When we use the variation for $J_{a}$ in eq.(2.22), we find - using partial integration and Stoke's theorem - that the equations of motion following from the three-dimensional WZ-term are equivalent to a term on the boundary of $\mathcal{M}_{3}$.

$$
\begin{aligned}
\delta S_{W Z} & =\frac{k}{6 \pi} \int_{\mathcal{M}_{3}} 3 \operatorname{Tr}[\delta J \wedge J \wedge J] \\
& =\frac{k}{6 \pi} \int_{\mathcal{M}_{3}} 3 \operatorname{Tr}[(\mathrm{d} \epsilon+[J, \epsilon]) \wedge J \wedge J] \\
& =\frac{k}{2 \pi} \int_{\mathcal{M}_{3}} \operatorname{Tr}[\epsilon \mathrm{d}(J \wedge J)]=\frac{k}{2 \pi} \int_{\Sigma_{2}} \epsilon \operatorname{Tr}[J \wedge J] .
\end{aligned}
$$

Here the commutator in the second line vanishes because of the cyclicity of the trace. We should therefore think of $\Sigma_{2}$ as a closed two-dimensional worldsheet, and $\mathcal{M}_{3}$ the volume enclosed by this surface. This means that though the WZ-term itself is three-dimensional, the equations of motion following from it only depend on the field configuration on boundary $\Sigma$ of the volume $\mathcal{M}_{3}$.

The resulting equations of motion for the total WZW-model are then of the form

$$
\left(\eta^{a b}-\epsilon^{a b}\right) \partial_{a} J_{b}=0
$$

\section{Example for SU(2)}

Let us very briefly return to the $S U(2) \cong S^{3}$ example we discussed earlier. For $\mathrm{SU}(2)$ the generators are given in eq.(2.25), and the structure constants are of the form

$$
f^{A B C}=i \sqrt{2} \epsilon^{A B C}
$$

To see the effect of the WZ-term on the target space geometry expressed in terms of coordinates, we identify the Maurer-Cartan form with the vielbeins on the target space geometry $J_{a}^{A}=$ $\partial_{a} X^{\mu} e_{\mu}^{A}$. We can use the Maurer-Cartan identity for a Lie group in eq.(2.24) $d J=-J \wedge J$ to rewrite the WZ-term as

$$
J \wedge J \wedge J=-J \wedge \mathrm{d} J=-\frac{1}{2} \mathrm{~d}(J \wedge J)
$$

We then see that the torsion $B_{\mu \nu}$ induced by the WZ-term on the target space is related to the commutator of the target space vielbeins, or equivalently the structure constants of the group.

The field strength $\mathrm{H}_{3}$ of the anti-symmetric field $B_{m u \nu}$ is obtained by its exterior derivative $H_{3}=\mathrm{d} B_{2}$. One can check that for $\mathrm{SU}(2)$ the $H_{3}$ flux will be proportional to

$$
H_{3}=\operatorname{vol} S^{3}
$$




\section{INTEGRABILITY AND SUPERSTRING THEORY}

\subsection{The Green-Schwarz Superstring}

So far we have only strings with only bosonic fields on their worldsheet. These bosonic strings suffer from various technical problems, the ground state is tachyonic and has a negative mass squared. This implies the vacuum is unstable. A problem that can be restored by including supersymmetry.

In the following section we will introduce the superstring. The introduction of supersymmetry on the worldsheet theory of the string can be done in several ways that can be shown to be equivalent:

- Ramond-Neveu-Schwarz (RNS) formalism is the original approach [16, 15], including fermions $\psi^{\mu}(\tau, \sigma)$ and supersymmetry on the string worldsheet. To make the resulting theory self-consistent and realise spacetime supersymmetry in the target space, one has to project out certain states. This is known as GSO projection [17].

- Green-Schwarz (GS) formalism [18] starts instead by considering strings that move on a supersymmetric target space. This is done by including target space fermions $\theta^{n}$ on the worldsheet. To ensure the resulting target space has the right amount of supersymmetry one has to require the worldsheet action to be invariant under an addition symmetry known as kappa-symmetry.

These different approaches can be convenient for different kinds of calculations. In this thesis we will focus on the Green-Schwarz approach, where the supersymmetry (and integrability) of the string directly manifest themselves in terms of the supersymmetric target space.

Just like we introduce the (bosonic) PCM and WZW-models valued on Lie groups in section 2.2 and 2.3 to threat the action for the bosonic string on symmetric spaces, we can consider these same actions on Lie supergroups, which describe the dynamics of a superstring on a corresponding supersymmetric target space.

In section 2.3 we saw the WZ-term ensures the action for the string world sheet is conformal. It furthermore induces torsion on the target space. The same is the case for the WZW-model on a supergroup. In the case of a supergroup however, the WZ-term enriches the worldsheet with an additional fermionic symmetry, known as kappa symmetry. In the following section we will introduce supermanifolds, supergroups, and the PCM and WZW-models on them. At the end we will return to this additional kappa-symmetry and examine it more closely.

\subsection{Supermanifolds}

Supermanifolds are a generalisation of Riemannian manifolds, where we include anticommuting fermionic directions $\theta^{\alpha}$, for which $\theta^{\alpha} \theta^{\beta}=-\theta^{\beta} \theta^{\alpha}$. Just like a $D$-dimensional Riemannian 
manifold $\mathcal{M}^{D}$ locally looks like $\mathbb{R}^{D}$, a supermanifold $\mathcal{M}^{(D \mid F)}$ has $D$ commting (bosonic) directions, and $F$ anti-commuting (fermionic) directions, and locally looks like $\mathbb{R}^{D} \times \mathbb{R}^{F}$. Since these fermionic coordinates anti-commute they differ from the bosonic coordinates in the following way

$$
\begin{array}{ll}
d X^{\mu} \otimes d X^{\nu}=d X^{\nu} \otimes d X^{\mu} & d X^{\mu} \wedge d X^{\nu}=-d X^{\nu} \wedge d X^{\mu} \\
d \theta^{\alpha} \otimes d \theta^{\beta}=-d \theta^{\beta} \otimes d \theta^{\alpha} & d \theta^{\alpha} \wedge d \theta^{\beta}=d \theta^{\beta} \wedge d \theta^{\alpha}
\end{array}
$$

We can combine the bosonic and fermionic coordinates into supercoordinates $Z^{A}=\left(X^{\mu}, \theta^{\alpha}\right)$. We will write the objects on the superspace with calligraphic capital letters, and Latin capital indices that range over both the bosonic directions as well as the fermionic directions. We can then decompose the supervielbein $\mathcal{E}_{M}^{A}$ into a bosonic and a fermionic part

$$
\mathcal{E}_{M}^{A}=\left(\begin{array}{c|c}
e_{\mu}^{a} & e_{\alpha}^{a} \\
\hline e_{\mu}^{\alpha} & e_{\alpha}^{\alpha}
\end{array}\right)
$$

So that

$$
\mathcal{E}^{A}=\mathcal{E}_{M}^{A} d Z^{M}=\left(\begin{array}{c}
e_{\mu}^{a} d x^{a}+e_{\alpha}^{a} d \theta^{\alpha} \\
e_{\mu}^{\alpha} d x^{\mu}+e_{\alpha}^{\alpha} d \theta^{\alpha}
\end{array}\right)=\left(\begin{array}{c}
\mathcal{E}^{a} \\
\mathcal{E}^{\alpha}
\end{array}\right)
$$

With these fermionic directions we can then generalise the conventional notions of a tangent space and a generalised metric $D_{\mu \nu}$ to a supertangent space and generalised supermetric $\mathcal{D}_{A B}$ (where for the moment we allow torsion terms in a generalised metric and do not yet promote them to be independent fields as we did earlier for the $B_{\mu \nu}$ field that carries the torsion of the bosonic generalised metric). The generalised supermetric will now decompose into bosonic and fermionic parts as

$$
\begin{aligned}
d s^{2} & =\mathcal{D}_{A B} d Z^{A} d Z^{B} \\
& =D_{a b} d x^{a} \otimes d x^{b}+D_{a \alpha} d x^{a} \otimes d \theta^{\alpha}+D_{\alpha \beta} d \theta^{\alpha} \otimes d \theta^{\beta} .
\end{aligned}
$$

In block form the generalised supermetric thus looks like

$$
d s^{2}=\left(d x^{a}, d \theta^{\alpha}\right)\left(\begin{array}{c|c}
D_{a b} & D_{a \beta} \\
\hline D_{\alpha b} & D_{\alpha \beta}
\end{array}\right)\left(\begin{array}{c}
d x^{b} \\
d \theta^{\beta}
\end{array}\right)
$$

Just like we can associate the torsion on a bosonic target space (the antisymmetric component of $D_{a b}$ ) with a $B_{2}$-field, we can associate another field (known as the fermion bispinor) $\mathbb{F}_{\alpha \beta}$ with a non-vanishing symmetric component of $D_{\alpha \beta}[19,20]$. Decomposing the generalised supermetric in this way so that the torsion can be thought of as independent fields we find

$$
\begin{aligned}
\mathcal{D}_{A B} d Z^{A} d Z^{B} & =G_{(a b)} d x^{a} \otimes d x^{b}+B_{[a b]} d x^{a} \wedge d x^{b} \\
& +G_{(a \alpha)} d x^{a} \otimes d \theta^{\alpha}+\Psi_{[a \alpha]} d x^{a} \wedge d \theta^{\alpha} \\
& +G_{[\alpha \beta]} d \theta^{\alpha} \otimes d \theta^{\beta}+F_{(\alpha \beta)} d \theta^{\alpha} \wedge d \theta^{\beta}
\end{aligned}
$$




\section{INTEGRABILITY AND SUPERSTRING THEORY}

Here we included the additional brackets to indicate that the bosonic part of the metric $G_{(a b)}$ is symmetric, the fermionic part of the metric $G_{[\alpha \beta]}$ is anti-symmetric, the bosonic torsion term $B_{[a b]}$ is anti-symmetric, and the fermionic torsion term $\mathbb{F}_{(\alpha \beta)}$ is symmetric.

Since the anti-symmetric gamma-matrices for a basis of all possible anti-symmetric terms in $D_{\alpha \beta}$ we can decompose the fermionic torsion $F_{(\alpha \beta)}$ onto a sum of gamma matrices times anti-symmetric Ramond-Ramond fields $F_{[a b . . .]}$. Note that even though these RR-fields tell us about the curvature in the fermionic part of the supermetric, these fields themselves are bosonic fields and only have Lorentz indices.

$$
F_{\alpha \beta}=F_{0}+\Gamma_{\alpha \beta}^{a} F_{a}+\frac{1}{2} \Gamma_{\alpha \beta}^{[a b]} F_{[a b]}+\frac{1}{3 !} \Gamma_{\alpha \beta}^{[a b c]} F_{[a b c]}+\frac{1}{4 !} \Gamma_{\alpha \beta}^{[a b c d]} F_{[a b c d]}+\ldots
$$

When we consider strings on a superspace with 10 bosonic directions we can choose to decompose the 32-component fermions into two Majorana-Weyl spinors of the same or different chiralities. This is known as the Type IIB and Type IIA description of the superstring, and results respectively in a superspace with either $\mathcal{N}=(2,0)$ or $\mathcal{N}=(1,1)$ supersymmetry. It turns out the Type IIB superspace will only have in odd RR-fluxes $\left(F_{1}, F_{3}, F_{5}\right.$ and their Hodge duals), while the Type IIA superspace only has even RR-fluxes $\left(F_{0}, F_{2}, F_{4}\right.$ and their Hodge duals).

Similarl to the way we can encode information about the torsion of the generalised supermetric into these RR-flieds, the off-diagonal component of the supermetric $G_{a \alpha}$ will always be symmetric. The torsion, or antisymmetric part $\Psi_{[a \alpha]}$ can be thought of as an additional field called the gravitino. In this section we aimed to give a brief introduction to the mathematica structure underlying supermanifolds. In the string theory literature it is convention to specify a supermanifold by giving the bosonic metric $G_{a b}$ together with the set of earlier described fields $B_{a b}, F_{a b \ldots}$, etc. that can be thought of as carrying the torsion on this space.

In terms of the supervielbein we can generalise the Polyakov action (2.16) to an action that couples the string to the metric of a supertarget space as

$$
S=\frac{1}{2 \pi \alpha^{\prime}} \int_{\Sigma} d^{2} z\left(\eta^{a b} \mathcal{E}_{M}^{A} \mathcal{E}_{N}^{B} \eta_{A B}+\epsilon^{a b} \mathcal{B}_{M N}(Z)\right) \partial_{a} Z^{M} \partial_{b} Z^{N}+\alpha^{\prime} \Phi(Z) \mathcal{R}(z)
$$

where the first term with the supervielbeins will result in the bosonic metric $\mathcal{G}_{(a b)}$ and antisymmetric (supertorsionless) part of the supermetric $\mathcal{G}_{[\alpha \beta]}$. In the above action we have chosen to set various fields, like the gravitino, equal to zero.

\section{An Example: The Supersphere}

Let us briefly illustrate an example of a supermanifold: the supersphere. Just like we defined the sphere to be congruent with the coset $S^{n} \cong S O(n+1) / S O(n)$ in section 2.2 .2 , we can define the supersphere

$$
S^{(n \mid 2 m)} \cong \frac{O S p(n+1 \mid 2 m)}{O S p(n \mid 2 m)}
$$


Where $O S p$ is the orthosymplectic supergroup. Analoguous to the normal sphere, we can think of a supersphere $S^{(n \mid 2)}$ as a submanifold in $\mathbb{R}^{(n+1 \mid 2)}$ of all the points in superspace $Z=\left(\vec{x}, \theta_{1}, \theta_{2}\right)$ that have a fixed distance to the origin.

$$
\eta^{a b} x^{a} x^{b}+\delta_{\alpha \beta} \theta^{\alpha} \theta^{\beta}=R^{2}
$$

The metric and vielbein can then be obtained using a stereographic projection of $S^{(2 \mid 2)}$ embedded in $\mathbb{R}^{(3 \mid 2)}$, analogous to the way one can obtain a metric for the stereographic projection of the Riemann sphere on the plane [19]. The supervielbein is

$$
\begin{aligned}
\mathcal{E}^{a} & =\frac{\sqrt{2}}{1+Z^{2}} d X^{a} \\
\mathcal{E}^{\alpha} & =\frac{i}{\left(1+Z^{2}\right)^{\frac{1}{2}}}\left(d \theta^{\alpha}-\frac{\theta^{\alpha} x_{a}}{1+Z^{2}} d x^{a}\right)
\end{aligned}
$$

with the metric given by

$$
d s^{2}=\delta_{a b} \mathcal{E}^{a} \mathcal{E}^{b}+i \epsilon_{\alpha \beta} \mathcal{E}^{\alpha} \mathcal{E}^{\beta}
$$

where we defined

$$
Z^{2}=\vec{x}^{2}+2 \theta_{1} \theta_{2}=R^{2}
$$

which in components reads

$$
\mathcal{G}_{A B}=\frac{2 \delta_{a b}}{\left(1+x^{2}\right)^{2}}\left(1-\frac{\epsilon_{\alpha \beta} \theta^{\alpha} \theta^{\beta}}{1+x^{2}}\right)
$$

Note that to zeroth order in bosonic coordinates we obtain the metric for the sphere $G_{a b}=$ $2 \delta_{a b}\left(1+x^{2}\right)^{-2}$ (the coordinates here are using a stereographic projection of the Riemann sphere). Also note this is a (torsionless) superspace, and the supermetric is symmetric in its bosonic components and anti-symmetric in its fermionic components, there are therefore no RamonRamond $B_{2}$, or gravitino fields present on this background.

Just to illustrate in more detail how one can extract the Ramond-Ramond fields from the supermetric, imagine we would change the fermionic part of the supermetric to include an additional symmetric term

$$
\mathcal{G}_{\alpha \beta}=\mathcal{G}_{[\alpha \beta]}^{(0)}+\frac{2 \delta_{\alpha \beta}}{\left(1+x^{2}\right)^{2}} \theta^{\alpha} \theta^{\beta}
$$

that we then associate with the fermion bispinor $\mathbb{F}_{\alpha \beta}$. When we consider the superspace $S^{(2 \mid 2)}$ with two bosonic, an two fermionic directions, the Pauli matrices form a complete basis for the possible anti-symmetric terms in $\mathcal{G}_{\alpha \beta}$. We can then decompose the symmetric component of $\mathcal{G}_{A B}$ into Ramond-Ramond fields as

$$
F_{(\alpha \beta)}=\frac{2 \delta_{\alpha \beta}}{\left(1+x^{2}\right)^{2}} \theta^{\alpha} \theta^{\beta}
$$




\section{INTEGRABILITY AND SUPERSTRING THEORY}

\subsection{Supergroups}

Analogous to how certain Lie groups can be seen as manifolds, we can construct super-Lie groups that can be identified with supermanifolds. A conventional matrix consists of a block of commuting numbers. By extension an (even) supermatrix (said to be of degree 0) is a block with numbers of the form

$$
\left(\begin{array}{c|c}
m & q \\
\hline p & n
\end{array}\right)
$$

Where the blocks $m$ and $n$ contain $m \times m$ and $n \times n$ matrices with commuting numbers, while the entries in the blocks $p$ and $q$ are respectively $n \times m$ and $m \times n$ matrices that contain anti-commuting (Grassmannian) numbers. For an odd supermatrix - of degree 1 - the anticommuting entries are on the diagonal, while the off-diagonal blocks contain normal commuting numbers instead, and one has to include the red terms in the expression below. For an even supermatrix these red terms will vanish. We will be mainly been considered with even supermatrices throughout this thesis.

We can define the supertrace, supertranspose and superdeterminant in a way similar to their 'normal' counterparts such that all the identities we know for these objects still hold

$$
\begin{aligned}
\operatorname{STr}(M) & =\operatorname{Tr}(m)-(-1)^{\operatorname{deg} M} \operatorname{Tr}(n) \\
M^{S T} & =\left(\begin{array}{cc}
m^{T} & (-1)^{\operatorname{deg} M} p^{T} \\
-(-1)^{\operatorname{deg} M} q^{T} & n^{T}
\end{array}\right) \\
\operatorname{sdet}(M) & =\frac{\operatorname{det}\left(m-q n^{-1} p\right)}{\operatorname{det}(n)}=\frac{\operatorname{det}(m)}{\operatorname{det}\left(n-p m^{-1} q\right)}
\end{aligned}
$$

Here $\operatorname{deg} M$ indicates the degree of the supermatrix.

We can decompose the generators of a supergroup into bosonic generators $B$ that are represented by matrices that have entries in the diagonal blocks $m$ and $n$, and fermionic generators $Q$ represented by matrices with entries in the off-diagonal blocks $p$ and $q$. We then see these generators satisfy commutation relations of the form

$$
[B, B] \subset B, \quad[B, Q] \subset Q, \quad[Q, Q] \subset B
$$

Note these commutation relations have a $\mathbb{Z}_{2}$ grading under which the bosonic generators are even and the fermionic generators are odd.

\subsubsection{Maurer-Cartan form on a Supergroup}

As we introduced in section 2.2, the PCM action on a Lie group can be written completely in terms of the Maurer-Cartan form on the group $J_{i}=g^{-1} \partial_{i} g$. To work out the Maurer-Cartan 
form of a supergroup we write the elements of the supergroup as $g=g^{(B)} e^{i \theta_{\alpha} Q^{\alpha}}$, with $Q^{\alpha}$ the fermionic generators. This gives us

$$
\begin{aligned}
J_{a} & =e^{-i \theta_{\alpha} Q^{\alpha}} g^{(B)} \partial_{a}\left(g^{(B)} e^{i \theta_{\alpha} Q^{\alpha}}\right) \\
& =e^{-i \theta_{\alpha} Q^{\alpha}}\left(J_{a}^{(B)}+i \partial_{a} \theta_{\alpha} Q^{\alpha}\right) e^{i \theta_{\alpha} Q^{\alpha}} \\
& =e^{-i \theta_{\alpha} Q^{\alpha}} \mathcal{D}_{a} e^{i \theta_{\alpha} Q^{\alpha}} \\
& =J_{a}^{(B)}+\mathcal{D}_{a} \Theta+\frac{1}{2}\left[\Theta, \mathcal{D}_{a} \Theta\right]+\ldots+\frac{1}{n !}\left[\Theta,\left[\Theta, \ldots\left[\Theta, \mathcal{D}_{a} \Theta\right] . .\right]\right],
\end{aligned}
$$

where we introduced the covariant derivative $\mathcal{D}_{a}=\partial_{a}+J_{a}^{(B)}$, used the shorthand notation $\Theta=-i \theta_{\alpha} Q^{\alpha}$, and used the superscript $(B)$ to emphasize that $g^{(B)}$ and $J^{(B)}$ are the group elements and Maurer-Cartan form on the bosonic subgroup of the supergroup. Here we used the Baker-Campbell-Hausdorf theorem in going to the last line.

Note the zeroth order terms in the fermions are equal to the Maurer-Cartan form on the bosonic subgroup of the supergroup. On a general supergroup it can be a daunting task to write out the full Maurer-Cartan form explicitly in terms of the fermionic coordinates as this might involve many higher order terms in the fermionic coordinates. It is therefore often more convenient to work with the Maurer-Cartan forms as fundamental fields on the string worldsheet directly, and not write them out explicitly in coordinates.

\subsubsection{PCM on a Supergroup}

Let us now briefly consider the principal chiral model on a supergroup, which will now be given in terms of supertrace over the Maurer-Cartan form on the full supergroup $J$ as

$$
S=\frac{k}{2 \pi} \int_{\Sigma} \mathrm{S} \operatorname{Tr}[J J]
$$

A supergroup always has at least a $\mathbb{Z}_{2}$ grading, as the bosonic generators form a subgroup and the commutators are of the form

$$
\begin{array}{cccc}
{[\mathfrak{b}, \mathfrak{b}] \subset \mathfrak{b},} & {[\mathfrak{b}, \mathfrak{f}] \subset \mathfrak{f},} & {[\mathfrak{f}, \mathfrak{f}] \subset \mathfrak{b}} \\
(0+0)=0 \bmod 2=0, & (0+1)=1 \bmod 2=1, & (1+1)=2 \bmod 2=0
\end{array}
$$

which means we can decompose the Maurer-Cartan form as

$$
J=J^{\mathfrak{b}} \oplus J^{\mathfrak{f}} .
$$

Here the $J^{\mathfrak{f}}$ are valued in the off-block-diagonal components of the supergroup. Since these $J^{\mathfrak{f}}$ have vanishing supertrace the PCM action will only contain terms quadratic on $J^{\mathfrak{f}}$ and decomposes as

$$
S=\frac{k}{2 \pi} \int_{\Sigma} d^{2} z \operatorname{STr}\left[J^{\mathfrak{b}} J^{\mathfrak{b}}+J^{\mathfrak{f}} J^{\mathfrak{f}}\right]
$$




\section{INTEGRABILITY AND SUPERSTRING THEORY}

where the first part describes the sigma model on the bosonic target space, and the second part describes the additional fermionic terms in the action.

\subsubsection{Graded Supergroups}

Analogous to how we introduced symmetric cosets in section 2.2.1, we can mod out a bosonic subgroup from a supergroup. For some cosets the $\mathbb{Z}_{2}$ grading of the coset will in that case combine with the fermionic $\mathbb{Z}_{2}$ grading to form a $\mathbb{Z}_{4}$ grading.

As an example, let us illustrate this for the maximally supersymmetric $\operatorname{AdS}_{5} \times \mathrm{S}^{5}$ solution of Type IIB supergravity, with a self-dual RR-flux $F_{5}=\operatorname{vol}\left(A d S_{5}\right)+\operatorname{vol}\left(S^{5}\right)$. This superspace is equivalent to the coset superspace

$$
\mathrm{AdS}_{5} \times \mathrm{S}^{5} \cong \frac{S U(2,2 \mid 4)}{S O(1,4) \times S O(5)} .
$$

Here $S U(2,2 \mid 4)$ is a supergroup with bosonic subgroups $S U(2,2) \cong S O(2,4)$ and $S U(4) \cong$ $S O(6)$ on its block-diagonal. We can represent the generators of the supergroup $S U(2,2 \mid 4)$ by the $8 \times 8$ complex supermatrices of the form

$$
X=\left(\begin{array}{c|c}
\mathfrak{s u}(2,2) & \theta \\
\hline \eta & \mathfrak{s u}(4)
\end{array}\right),
$$

where $\mathfrak{s u}(2,2)$ and $\mathfrak{s u}(4)$ are the even (bosonic) $4 \times 4$ matrices respectively containing the generators of these respective (bosonic) groups. The $\theta$ and $\eta$ are the $4 \times 4$ odd matrices whose elements are linear in the fermionic variables.

Analogous to how we earlier introduced the $\mathbb{Z}_{2}$ graded coset spaces in section 2.2 .2 , we can define an automorphism $\Omega$ for which $\Omega^{4}=\mathbb{I}$, that acts on these generators as $\Omega(X)=-K X^{S T} K^{-1}$, with

$$
K=\operatorname{diag}(\mathcal{K}, \mathcal{K}), \quad \text { with } \quad \mathcal{K}=\left(\begin{array}{cccc}
0 & -1 & 0 & 0 \\
1 & 0 & 0 & 0 \\
0 & 0 & 0 & -1 \\
0 & 0 & 1 & 0
\end{array}\right) .
$$

This automorphism now allows us to decompose the generators of $S U(2,2 \mid 4)$ into a direct sum of four subspaces, where each subspace is defined to be an eigenspace of $\Omega$,

$$
\Omega\left(\mathfrak{g}^{(k)}\right)=i^{k} \mathfrak{g}^{(k)}, \quad \text { where } \quad \mathfrak{g}^{(k)}=\frac{1}{4}\left(\mathfrak{g}+i^{3 k} \Omega(\mathfrak{g})+i^{2 k} \Omega^{2}(\mathfrak{g})+i^{k} \Omega^{3}(\mathfrak{g})\right) .
$$

giving

$$
\mathfrak{g}=\mathfrak{g}^{(0)} \oplus \mathfrak{g}^{(1)} \oplus \mathfrak{g}^{(2)} \oplus \mathfrak{g}^{(3)}
$$


such that

$$
\left[\mathfrak{g}^{(i)}, \mathfrak{g}^{(j)}\right] \subseteq \mathfrak{g}^{(i+j)} \bmod \mathbb{Z}_{4}, \quad i, j=0,1,2,3
$$

These different subspaces will take on the form

$$
\begin{aligned}
\mathfrak{g}^{(0)} & =\frac{1}{2}\left(\begin{array}{c|c}
m-K m^{t} K^{-1} & 0 \\
\hline 0 & n-K n^{t} K^{-1}
\end{array}\right), \\
\mathfrak{g}^{(2)} & =\frac{1}{2}\left(\begin{array}{c|c}
m+K m^{t} K^{-1} & 0 \\
\hline 0 & n+K n^{t} K^{-1}
\end{array}\right) \\
\mathfrak{g}^{(1)} & =\frac{1}{2}\left(\begin{array}{c|c}
0 & \theta-i K \eta^{T} K^{-1} \\
\hline \eta+i K \theta^{T} K^{-1} & 0
\end{array}\right), \\
\mathfrak{g}^{(3)} & =\frac{1}{2}\left(\begin{array}{c|c}
0 & \theta+i K \eta^{T} K^{-1} \\
\hline \eta-i K \theta^{T} K^{-1} & 0
\end{array}\right) .
\end{aligned}
$$

where $K$ is a $4 \times 4$ matrix for which $\Omega(X)=-K X^{S T} K^{-1}$.

\subsubsection{WZW-model on Supergroups}

If we consider the WZW-model on a symmetric superspace (that is a coset of a super Lie group with a $\mathbb{Z}_{4}$ grading (see section 2.6.3)), we can analogously to section 2.2.1 gauge out the bosonic subgroup $H \in \mathfrak{g}^{(0)}$. Since $H$ is a bosonic subgroup, the remaining bosonic part of the PCM action contains $J^{(2)}$, analoguous to the bosonic coset in section 2.2.1.

The WZ-term on a $\mathbb{Z}_{4}$ graded supercoset cannot depend on $J^{(0)}$ as we gauge out this subgroup, and the only relevant contributions can therefore come from

$$
\mathrm{S} \operatorname{Tr}\left[\left(J^{(1)}+J^{(2)}+J^{(3)}\right) \wedge\left(J^{(1)}+J^{(2)}+J^{(3)}\right) \wedge\left(J^{(1)}+J^{(2)}+J^{(3)}\right)\right]
$$

It turns out the WZ-term can in that case be written as [9]

$$
\operatorname{STr}\left[J^{(2)} \wedge\left(J^{(1)} \wedge J^{(1)}-J^{(3)} \wedge J^{(3)}\right]=d \operatorname{STr}\left[J^{(1)} \wedge J^{(3)}\right],\right.
$$

where we used the Maurer-Cartan identity $d J_{a}^{(i+j)}=\left[J_{a}^{(i)}, J_{b}^{(j)}\right]$. The WZ-term can thus be written following eq.(2.60) as

$$
S_{W Z}=\frac{k}{2 \pi} \int_{\Sigma} d^{2} z \epsilon^{a b} \operatorname{STr}\left[J_{a}^{(1)} J_{a}^{(3)}\right]
$$

So that the full WZW-action on a supergroup can be written as

$$
S_{W Z W}=\frac{k}{2 \pi} \int_{\Sigma} d^{2} z \eta^{a b} \mathrm{~S} \operatorname{Tr}\left[J_{a}^{(2)} J_{b}^{(2)}\right]+\kappa \epsilon^{a b} \mathrm{~S} \operatorname{Tr}\left[J_{a}^{(1)} J_{b}^{(3)}\right]
$$




\section{INTEGRABILITY AND SUPERSTRING THEORY}

where the parameter $\kappa$ has to be fixed to ensure kappa symmetry on the worldsheet action (we will come back to this in the next section). The equations of motion, along with the Maurer-Cartan identity can then be written (in lightcone coordinates) as

$$
\begin{aligned}
D_{+} J_{-}^{(2)}+\left[J_{+}^{(1)}, J_{-}^{(1)}\right] & =0 \\
D_{-} J_{+}^{(2)}+\left[J_{-}^{(3)}, J_{+}^{(3)}\right] & =0 \\
\partial_{+} J_{-}^{(0)}-\partial_{-} J_{+}^{(0)}+\left[J_{+}^{(0)}, J_{-}^{(0)}\right]+\left[J_{+}^{(2)}, J_{-}^{(2)}\right]+\left[J_{+}^{(3)}, J_{-}^{(1)}\right]+\left[J_{+}^{(1)}, J_{-}^{(3)}\right] & =0 \\
D_{+} J_{-}^{(1)}-D_{-} J_{+}^{(1)}+\left[J_{+}^{(3)}, J_{-}^{(2)}\right] & =0 \\
D_{+} J_{-}^{(3)}-D_{-} J_{+}^{(3)}+\left[J_{+}^{(2)}, J_{-}^{(1)}\right] & =0 \\
{\left[J_{+}^{(1)}, J_{-}^{(2)}\right] } & =\left[J_{+}^{(2)}, J_{-}^{(3)}\right]=0,
\end{aligned}
$$

where $D_{a} \cdot=\partial_{a}+\left[J_{a}^{(0)}, \cdot\right]$. Note that, using the commutation relations in eq.(2.93) all terms in each line have the same grading. These equations of motion can now be obtained from the flatness condition for a Lax connection

$$
L_{ \pm}=J_{ \pm}^{(0)}+z J_{ \pm}^{(1)}+z^{\mp 2} J_{ \pm}^{(2)}+z^{-1} J_{ \pm}^{(3)} .
$$

\subsubsection{Kappa Symmetry}

Let us now return to the kappa-symmetry for the Green-Schwarz superstring. To study this in more detail, we will first have a look at the action for the Green-Schwarz superstring on flat Minkowski superspace. When written explicitly in terms of coordinates $X^{\mu}$ and $\theta^{\alpha A}$ the action is of the form

$$
\begin{aligned}
& S_{G S}^{\mathrm{Mink}}=\frac{-1}{4 \pi \alpha^{\prime}} \int_{\Sigma} d^{2} x \frac{1}{2} \eta^{a b} \eta_{\mu \nu} \Pi_{a}^{\mu} \Pi_{b}^{\nu}+S_{W Z}^{\mathrm{Mink}}, \\
& \text { where } \quad \Pi_{a}^{\mu}=\partial_{a} X^{\mu}-\bar{\theta}^{A \alpha} \Gamma_{\alpha \beta}^{\mu} \partial_{a} \theta^{\beta A},
\end{aligned}
$$

where we wrote the target space coordinates $Z^{A}$ on the super-Minkowski space explicitly in terms of both the bosonic coordinates $X^{\mu}(\sigma, \tau)$ and the additional fermionic coordinates $\theta^{\alpha}(\sigma, \tau)$.

For the worldsheet action to be supersymmetric it needs to be invariant under the transformation

$$
\delta X^{\mu}=i \bar{\theta}^{A \alpha} \Gamma_{\alpha \beta}^{\mu} \delta \theta^{A \beta}, \quad \delta \theta^{A \alpha}=2 i \Gamma_{\alpha \beta}^{\mu} \Pi_{\mu} \kappa^{A \alpha}
$$

where $\kappa$ is an arbitrary spinor. This symmetry reduces the number of physical fermionic degrees of freedom by half, from 32 down to 16 . 
This invariance of the action can be achieved by adding an additional term, usually referred to as the Wess-Zumino (or Chern-Simons) term. For the WZW-model on super-Minkowski space this WZ-term is of the form

$$
S_{W Z}^{\mathrm{Mink}}=\frac{-1}{4 \pi \alpha^{\prime}} \int_{\Sigma} d^{2} x \epsilon^{a b}\left[-\partial_{a} X^{\mu}\left(\bar{\theta}^{1} \Gamma_{\mu} \partial_{b} \theta^{1}-\bar{\theta}^{2} \Gamma_{\mu} \partial_{b} \theta^{2}\right)-\bar{\theta}^{1} \Gamma^{\mu} \partial_{a} \theta^{1} \bar{\theta}^{2} \Gamma_{\mu} \partial_{b} \theta^{2}\right] .
$$

Finding this additional term makes it more difficult to write down the Green-Schwarz action on an arbitrary supergeometry. If we were to replace $\eta_{\mu \nu}$ in eq.(2.101) with an arbitrary target space metric $G_{\mu \nu}$, the additional Wess-Zumino term has to be amended appropriately to preserve the $\kappa$-symmetry of the action. Writing down the GS action for the string, with the appropriate WZ-term on an arbitrary background can therefore be a convoluted task.

Fortunately, when we obtain the action for the superstring as a WZW-model on a supergroup, it is exactly the WZ-term that gives the correct $\kappa$-symmetry to the worldsheet action![24]

This action of this $\kappa$-symmetry can be understood as identifying points the supergroup, that are related by the right action with a fermionic element $e^{\theta Q}$ [23], where $Q$ is a fermionic generator. Identifying these elements suggests the WZW-model on a supergroup $G$, means that we are considering the coset $G / \tilde{Q}$, where $\tilde{Q}$ includes a subset of the fermionic generators.

\subsection{Supergravity}

So far we have been mainly concerned with the classical action for the superstring. The requirement that beta function for the string vanishes on an arbitrary target space imposes constraints on the background fields that couple to the string worldsheet. If we consider just the bosonic sector of the string (with target space fields $G_{\mu \nu}, B_{\mu \nu}$ and $\Phi$ ), the one-loop beta function for the sigma model in eq.(2.16) is

$$
\begin{aligned}
\beta^{G_{\mu \nu}} & =\alpha^{\prime}\left[R_{\mu \nu}-2 \nabla_{\mu} \nabla_{\nu} \phi+\frac{1}{4} H_{\mu \kappa \sigma} H_{\nu}^{\kappa \sigma}\right]+\mathcal{O}\left(\alpha^{\prime 2}\right) \\
\beta^{B_{\mu \nu}} & =\alpha^{\prime}\left[-\frac{1}{2} \nabla^{\kappa} H_{\kappa \mu \nu}+\nabla^{\kappa} \Phi H_{\kappa \mu \nu}\right]+\mathcal{O}\left(\alpha^{\prime 2}\right) \\
\beta^{\Phi} & =\alpha^{\prime}\left[\frac{D-26}{6 \alpha^{\prime}}-\frac{1}{2} \nabla^{2} \Phi+\nabla_{\kappa} \Phi \nabla^{\kappa} \Phi-\frac{1}{24} H_{\kappa \mu \nu} H^{\kappa \mu \nu}\right]+\mathcal{O}\left(\alpha^{\prime 2}\right)
\end{aligned}
$$

These constraints can be interpreted as equations of motion that the fields $G_{\mu \nu}, B_{\mu \nu}$ and $\Phi$ have to satisfy for the string theory on this target space to be consistent. These equations of motion can be obtained from an action that consists of the Einstein-Hilbert action, coupled to 


\section{INTEGRABILITY AND SUPERSTRING THEORY}

a scalar field and an anti-symmetric three form field strength $H_{3}=\mathrm{d} B_{2}$

$$
S^{\mathrm{NS}}=\frac{1}{168 \pi G_{10}} \int d^{10} x \sqrt{-G} e^{-2 \Phi}\left(R+4 \partial_{\mu} \Phi \partial^{\mu} \Phi-\frac{1}{12} H_{\mu \nu \rho} H^{\mu \nu \rho}\right) .
$$

with $G_{10}$ the ten dimensional Newton's constant.

If we calculate higher loop corrections to the beta function, we would find higher order corrections that contain higher derivatives of the curvature, $H_{\mu \nu \rho}$ field strength, and dilaton. We can thus think of this action as a low energy effective action for the string backgrounds, that is accurate when the curvature, and derivatives of $H_{\mu \nu \rho}$ and $\Phi$ are relatively small. Note the higher order corrections also come with higher orders of $\alpha^{\prime}$. This implies the low energy effective action is valid up to energies of order the string mass $m_{s} \sim \alpha^{\prime-1} \sim \ell^{-2}$.

\subsubsection{Including RR-fluxes}

When we do the same analysis for the string on a superspace we find similar results, where the vanishing of the beta function now implies the worldsheet action is not only conformal, but also kappa symmetric [21, 22]. The constraints for the vanishing of the beta function will now include the fermionic directions of the target space, which (as we showed in section 2.5) can now be encoded in terms of Ramond-Ramond fields, as well as other fields that carry spinor indices.

Depending on whether one considers a Type IIA or IIB superspace (with the fermionic directions described by Majorana-Weyl spinors of opposing or equal chiralities) this leads to backgrounds with only even or odd RR-fluxes.

If we set all fermionic field equal to zero, the backgrounds have to satisfy the supergravity equations.

\section{Type IIA Supergravity}

For Type IIA supergravity the action contains the additional terms in the action are

$$
S_{\mathrm{IIA}}=S^{\mathrm{NS}}+S_{\mathrm{IIA}}^{\mathrm{R}}+S_{\mathrm{IIA}}^{\mathrm{CS}}
$$

where

$$
\begin{aligned}
& S_{\mathrm{IIA}}^{\mathrm{R}}=\frac{-1}{4 \kappa_{10}^{2}} \int d^{10} x\left(F_{(0)}^{2}+\frac{1}{2} F_{(2)}^{2}+\frac{1}{4 !} F_{(4)}^{2}\right) \\
& S_{\mathrm{IIA}}^{\mathrm{CS}}=\frac{-1}{4 \kappa_{10}^{2}} \int d^{10} x\left(d C_{(3)} \wedge d C_{(3)} \wedge B_{(2)}+\frac{1}{3} F_{(0)} d C_{(3)} \wedge B_{(2)}^{3}+\frac{1}{20} F_{(0)}^{2} \wedge B_{(2)}^{5}\right) .
\end{aligned}
$$


Here the RR-field strengths are related to their potential as

$$
\begin{aligned}
& F_{(2)}=d C_{(1)}+F_{(0)} \wedge B_{(2)}, \\
& F_{(4)}=d C_{(3)}-H_{(3)} \wedge C_{(1)}+\frac{1}{2} F_{(0)} \wedge B_{(2)} \wedge B_{(2)} .
\end{aligned}
$$

\section{Type IIB Supergravity}

Analoguously, the complete Type IIB supergravity action is given by

$$
S_{\mathrm{IIB}}=S^{\mathrm{NS}}+S_{\mathrm{IIB}}^{\mathrm{R}}+S_{\mathrm{IIB}}^{\mathrm{CS}}
$$

where

$$
\begin{gathered}
S_{\mathrm{IIB}}^{\mathrm{R}}=\frac{-1}{4 \kappa_{10}^{2}} \int d^{10} x\left(F_{(1)}^{2}+\frac{1}{3 !} F_{(3)}^{2}+\frac{1}{2} \frac{1}{5 !} F_{(3)}^{2}\right) \\
S_{\mathrm{IIB}}^{\mathrm{CS}}=-\frac{1}{4 \kappa_{10}^{2}} \int d^{10} x C_{(4)} \wedge H_{(3)} \wedge d C_{(2)}
\end{gathered}
$$

and where the potentials are related to the field strengths as

$$
\begin{aligned}
& F_{(1)}=d C_{(0)} \\
& F_{(3)}=d C_{(2)}-C_{(0)} \wedge H_{(3)} \\
& F_{(5)}=d C_{(4)}-C_{(2)} \wedge H_{(3)}
\end{aligned}
$$

Here in addition, it has to be imposed by hand that $F_{(5)}=\star F_{(5)}$.

\section{Equations of Motion}

The resulting equations of motion for Type IIA and Type IIB are

$$
\begin{aligned}
R_{\mu \nu}+2 \nabla_{\mu} \nabla_{\nu} \Phi-\frac{1}{2} H_{\mu \sigma} H_{\nu}{ }^{\sigma}-\frac{1}{4} e^{2 \Phi} \sum_{p=0}^{5} F_{\mu}^{(p)} F_{\nu}^{(p)} & =0 \\
\left(\mathrm{~d}+H_{(3)} \wedge\right) \star \sum_{p} F_{(p)} & =0 \\
\mathrm{~d}\left(e^{-2 \Phi} \star H_{(3)}\right)-\frac{1}{2} \sum_{p}\left(\star F_{(p)} \wedge F_{(p-2)}\right) & =0 \\
2 \nabla^{2} \Phi-(\nabla \Phi)^{2}+\frac{1}{4} R-\frac{1}{8} H_{(3)}^{2} & =0
\end{aligned}
$$




\section{INTEGRABILITY AND SUPERSTRING THEORY}

where $F_{\mu}^{(p)} F_{\nu}^{(p)}$ implies the $p$-form contracted with itself over all but one index, and $H_{(3)}$ implies a contraction of $H_{\mu \nu \rho}$ with itself. Here $p=2 k$ and is even for Type IIA, and $p=2 k+1$ and is odd for Type IIB.

\subsection{T-Duality}

In the following sections we will introduce the ideas of both Abelian and non-Abelian T-duality, and its relation with integrability. T-duality (or target-space duality) is an equality between the worldsheet theory of the string on a target space with a compact circular direction of radius $R$, and one with a circular direction of radius $R^{-1}$.

More generally it states that the full quanutum theory of a string on a target space with a compact isometry direction, metric $G_{\mu \nu}$, B-field $B_{\mu \nu}$, and dilaton $\Phi$ is equivalent to the string on a different target space, with metric $\bar{G}_{\mu \nu}$, B-field $\bar{B}_{\mu \nu}$ and dilaton $\bar{\Phi}$, related to to old ones by the Buscher rules in eq.(2.116). If we also consider RR-fluxes on the original background, they will transform according to (2.124).

In the non-Abelian case we start from a target space with a non-Abelian isometry group, and find - following the same procedure - a target space related to the original one by the generalised Buscher rules in eq.(2.133). In the Abelian case the full quantum theory of the string on the two dual spaces are equivalent. For the non-Abelian T-duality, this is no longer the case and the full quantum theory of the string is not equivalent on the dual target spaces.

Non-Abelian T-duality does however bring us from one target space on which the worldsheet action of the string is conformal to another one where this is also the case. Both the original and the 'dual' target spaces will therefore be solutions of supergravity. This means the non-Abelian T-duality can be used as a 'solution generating technique', starting from a highly symmetric target space one can obtain more complicated supergravity solutions, where the original isometry group on which we performed the NATD is now broken. We will illustrate this further in chapter 7 .

The 'dual' supergravity solutions that are obtained by performing a (non-Abelian) T-duality on a more symmetric solution are in general more complicated and less supersymmetry, as various isometries are broken during this procedure. However, (non-Abelian) T-duality preserves the integrable structure of the string worldsheet. This is because the action of a T-duality on the worldsheet can be seen as a canonical transformation in phase space, we discuss this in section 2.8.3.

\subsubsection{The Buscher Rules}

To derive these results we follow $[25,26]$, and start from a target space with a compact $U(1)$ isometry direction $X^{\theta}$, and denote $\partial_{a} X^{\theta}=J_{a}^{\theta}$, so we can decompose the terms in the bosonic 
sector of the $\sigma$-model as

$$
\begin{aligned}
\eta^{a b} G_{\mu \nu} \partial_{a} X^{\mu} \partial_{b} X^{\nu} & =\eta^{a b}\left(G_{\theta \theta} J_{a}^{\theta} J_{b}^{\theta}+G_{\theta \rho} J_{a}^{\theta} \partial_{b} X^{\rho}+G_{\rho \sigma} \partial_{a} X^{\rho} \partial_{b} X^{\sigma}\right) \\
\epsilon^{a b} B_{\mu \nu} \partial_{a} X^{\mu} \partial_{b} X^{\nu} & =\epsilon^{a b}\left(B_{\theta \rho} J_{a}^{\theta} \partial_{b} X^{\rho}+B_{\rho \sigma} \partial_{a} X^{\rho} \partial_{b} X^{\sigma}\right)
\end{aligned}
$$

where the indices $\rho$ and $\sigma$ range over the remaining directions that do not involve $X^{\theta}$. Note that $J_{a}^{\theta}$ here is the Maurer-Cartan form on the U(1) isometry direction, following the notation we introduced earlier in section 2.2. Inserting this separation of the $\theta$-direction in the bosonic $\sigma$-model

$$
S=\frac{1}{2 \pi \alpha^{\prime}} \int_{\Sigma} d^{2} z\left(\eta^{a b} G_{\mu \nu}+\epsilon^{a b} B_{\mu \nu}\right) \partial_{a} X^{\mu} \partial_{b} X^{\nu}+\alpha^{\prime} \Phi(X) R(z)
$$

then gives us

$$
\begin{array}{r}
S\left[X^{\rho}, J_{a}\right]=\frac{1}{2 \pi \alpha^{\prime}} \int_{\Sigma} d^{2} z \eta^{a b}\left(G_{\theta \theta} J_{a}^{\theta} J_{b}^{\theta}+G_{\theta \rho} J_{a}^{\theta} \partial_{b} X^{\rho}+G_{\rho \sigma} \partial_{a} X^{\rho} \partial_{b} X^{\sigma}\right) \\
+\epsilon^{a b}\left(B_{\theta \rho} J_{a}^{\theta} \partial_{b} X^{\rho}+B_{\rho \sigma} \partial_{a} X^{\rho} \partial_{b} X^{\sigma}\right)+\alpha^{\prime} \Phi R
\end{array}
$$

We now promote the global $U(1)$ isometry of the $X^{\theta}$-direction to a local gauge symmetry on the string worldsheet by replacing the derivatives $J_{a}^{\theta}=\partial_{a} X^{\theta} \rightarrow D_{a} X^{\theta}=\partial_{a} X^{\theta}+A_{a} \cdot{ }^{1}$ We then add a Lagrange multipier term $S_{L M}$ to the action,

$$
S_{L M}=\frac{1}{2 \pi \alpha^{\prime}} \int_{\Sigma} d z^{2} \bar{\theta} \epsilon^{a b} \partial_{a} A_{b}
$$

with $\bar{\theta}$ a Lagrange multiplier field. The equations of motion for $\bar{\theta}$ now imply $\epsilon^{a b} \partial_{a} A_{b}=F_{a b}=0$. This forces the field strength to vanish, and ensures that $A_{a}$ is a flat connection $A_{a}=\partial_{a} \lambda$. With the introduction of this Lagrange multiplier term, the gauge field does not add additional degrees of freedom to the system.

Since the gauge connection is flat, $J_{a}^{\theta}=D_{a} X^{\theta}=\partial_{a} X^{\theta}+A_{a}=\partial_{a}\left(X^{\theta}+\lambda\right)$. The different gauge choices for $\lambda$ now correspond to a coordinate transformation along the $\theta$-direction, $\theta \rightarrow$ $\theta+\lambda$. It is easiest to work with a gauge for the connection where we set $\lambda=\phi-X^{\theta}$, so that $J_{a}^{\theta}=A_{a}$, which we will do in the remainder of this section.

\footnotetext{
${ }^{1}$ Here we write $X^{\theta}, J_{a}^{\theta}$ where the index $\theta$ only ranges over the single $\mathrm{U}(1)$ isometry direction. We choose to omit this index on the gauge field $A_{a}^{\theta}$ in this section, and simply write $A_{a}$ as the gauge field is Abelian. We choose this notation where the group index is made explicit on the Maurer-Cartan form to make it easier for the reader when we later generalise the results in this section to the non-Abelian case, where $X^{i}$ and $J_{a}^{i}$ have a non-Abelian index $i$ that runs over multiple generators.
} 


\section{INTEGRABILITY AND SUPERSTRING THEORY}

In this notation, the full partition function is written - after partial integration on the Lagrange multiplier term - as

$$
\begin{aligned}
& Z=\int \mathcal{D} X^{\rho} \mathcal{D} A_{a} \mathcal{D} \bar{\theta} e^{-i S\left[X^{\rho}, A_{a}, \bar{\theta}\right]}, \quad \text { with } \\
& \begin{array}{r}
S\left[X^{\rho}, A_{a}, \bar{\theta}\right]=\frac{1}{2 \pi \alpha^{\prime}} \int_{\Sigma} d^{2} z \quad A_{a}\left(\eta^{a b} G_{\theta \theta}\right) A_{b} \\
+2\left(\eta^{a b} G_{\theta \rho} \partial_{a} X^{\rho}+\epsilon^{a b} B_{\theta \rho} \partial_{a} X^{\rho}-\epsilon^{a b} \partial_{a} \bar{\theta}\right) A_{b} \\
\quad+\eta^{a b} G_{\rho \sigma} \partial_{a} X^{\rho} \partial_{b} X^{\sigma}+B_{\rho \sigma} \partial_{a} X^{\rho} \partial_{b} X^{\sigma}+\alpha^{\prime} \Phi R
\end{array}
\end{aligned}
$$

Integrating out $\bar{\theta}$ brings us back to the partition function in eq.(2.111). To obtain the T-dual model we instead integrate out $A_{a}$, which leaves us with

$$
\begin{aligned}
& Z=\frac{1}{\sqrt{G_{\theta \theta}}} \int \mathcal{D} X^{\rho} \mathcal{D} \bar{\theta} e^{-i S\left[X^{\rho}, \bar{\theta}\right]}, \\
& S\left[X^{\rho}, \bar{\theta}\right]=\frac{1}{2 \pi \alpha^{\prime}} \int_{\Sigma} d^{2} z \eta^{a b} G_{\rho \sigma} \partial_{a} X^{\rho} \partial_{b} X^{\sigma}+B_{\rho \sigma} \partial_{a} X^{\rho} \partial_{b} X^{\sigma}+\alpha^{\prime} \Phi R+ \\
& \left(\eta^{a b} G_{\theta \rho} \partial_{a} X^{\rho}+\epsilon^{a b} B_{\theta \rho} \partial_{a} X^{\rho}-\epsilon^{a b} \partial_{a} \bar{\theta}\right)\left(\eta_{b c} G_{\theta \theta}^{-1}\right)\left(\eta^{c d} G_{\theta \kappa} \partial_{c} X^{\kappa}+\epsilon^{c d} B_{\theta \kappa} \partial_{c} X^{\kappa}-\epsilon^{c d} \partial_{c} \bar{\theta}\right)
\end{aligned}
$$

Where we can now collect all the crossterms of the above product to define a new target space metric $\bar{G}_{\mu \nu}$ and B-field $\bar{B}_{\mu \nu}$. The factor ${\sqrt{G_{\theta \theta}}}^{-1}$ in the partition function can be absorbed in a redefinition of the dilaton.

$$
\begin{aligned}
\frac{1}{4 \pi \alpha^{\prime}} \int_{\Sigma} d^{2} z \eta^{a b}\left(\bar{G}_{\bar{\theta} \bar{\theta}} \partial_{a} \bar{\theta} \partial_{b} \bar{\theta}+\right. & \left.2 \bar{G}_{\bar{\theta} \mu} \partial_{a} \bar{\theta} \partial_{b} X^{\mu}+\bar{G}_{\mu \nu} \partial_{a} X^{\mu} \partial_{b} X^{\nu}\right)+ \\
& +\epsilon^{a b}\left(2 \bar{B}_{\bar{\theta} \mu} \partial_{a} \bar{\theta} \partial_{b} X^{\mu}+\bar{B}_{\mu \nu} \partial_{a} X^{\mu} \partial_{b} X^{\nu}\right)+\alpha^{\prime} \Phi(X) R(z)
\end{aligned}
$$

The partition functions for the actions in eqs.(2.110) and (2.115) now describe a closed string on two different spaces. These two different partition functions are however identical as we have shown, and one can go from one to the other by redefining the fields. The transformation rules for the target space geometry before and after the duality are known as the Buscher rules $[25,26]$.

$$
\begin{aligned}
\bar{D}_{\mu \nu} & =\left(\begin{array}{c|c}
D_{\mu \nu}-D_{\mu \theta} G_{\theta \theta}^{-1} D_{\theta \nu} & -D_{\mu \theta} G_{\theta \theta}^{-1} \\
\hline G_{\theta \theta}^{-1} D_{\theta \nu} & G_{\theta \theta}^{-1}
\end{array}\right), \\
\bar{\Phi} & =\Phi-\frac{1}{2} \ln \left(G_{\theta \theta}\right),
\end{aligned}
$$

where we now combine $D_{\mu \nu}=G_{\mu \nu}+B_{\mu \nu}$. The components of the metric and $B$-field that only involve the other directions $\rho$ and $\sigma$ are unaffected. 
Similarly we can find the transformation rules for the generalised vielbeins under a T-duality. To obtain the dual action we had to integrate out $A_{a}$. This is equivalent to obtaining the equations of motion for $A_{a}$ and inserting them into the action. For the action in eq.(2.113) these are

$$
\begin{aligned}
& \delta A_{+}=G_{\theta \theta} A_{-}+D_{\rho \theta} \partial_{-} X^{\rho}+\partial_{-} \bar{\theta}=0, \\
& \delta A_{-}=G_{\theta \theta} A_{+}+D_{\theta \rho} \partial_{+} X^{\rho}-\partial_{+} \bar{\theta}=0,
\end{aligned}
$$

where we wrote the worldsheet coordinates in light-cone gauge. Since we can choose a convenient gauge where that $J_{a}^{\theta}=A_{a}$ this then implies that the initial Maurer-Cartan forms $J_{a}^{\theta}$ on the isometry direction, will transform after a T-duality as

$$
\begin{aligned}
& J_{+} \rightarrow \frac{1}{G_{\theta \theta}}\left(\partial_{+} \bar{\theta}-D_{\theta \rho} \partial_{+} X^{\rho}\right) \\
& J_{-} \rightarrow \frac{-1}{G_{\theta \theta}}\left(\partial_{-} \bar{\theta}+D_{\rho \theta} \partial_{-} X^{\rho}\right) .
\end{aligned}
$$

\subsubsection{Transformation of the RR-fluxes}

So far we discussed how the NS-sector of the supergravity background transforms under a Tduality. We will now focuss on the way the RR-sector transforms when we perform a T-duality on a bosonic isometry direction.

A first way to see how a super-targetspace deforms under a T-duality is by considering a generalisation of the Buscher rules (2.116) to those for a superspace, following section 2.5. This then gives us

$$
\overline{\mathcal{D}}_{A B}=\left(\begin{array}{c|c}
\mathcal{D}_{A B}-\frac{\mathcal{D}_{A \theta} \mathcal{D}_{\theta B}}{G_{\theta \theta}} & -\frac{D_{A \theta}}{G_{\theta \theta}} \\
\hline \frac{\mathcal{D}_{\theta B}}{G_{\theta \theta}} & G_{\theta \theta}^{-1}
\end{array}\right)
$$

Here we simply applied to same Buscher rules as before, but now have capital indices $A, B$ that run over both the bosonic directions (indicated by small Latin indices $a, b$ ) and fermionic directions (indiated by small Greek indices $\alpha, \beta)$ of the super targetspace.

As we explained in section 2.5, we can combine the RR-fields to form the fermion bispinor $F_{\alpha \beta}$ that we can think of as carrying the torsion term of the purely fermionic part of the supermetric

$$
F_{\alpha \beta}=F_{0}+\Gamma_{\alpha \beta}^{a} F_{a}+\frac{1}{2} \Gamma_{\alpha \beta}^{[a b]} F_{[a b]}+\frac{1}{3 !} \Gamma_{\alpha \beta}^{[a b c]} F_{[a b c]}+\frac{1}{4 !} \Gamma_{\alpha \beta}^{[a b c d]} F_{[a b c d]}+\ldots
$$

To see how the fermion part of the generalised supermetric transforms under a T-duality we can take the part of eq.(2.116) where $A$ and $B$ only run over the fermionic directions

$$
\overline{\mathcal{D}}_{\alpha \beta}=\mathcal{D}_{\alpha \beta}-\frac{\mathcal{D}_{\alpha \theta} \mathcal{D}_{\theta \beta}}{G_{\theta \theta}} .
$$




\section{INTEGRABILITY AND SUPERSTRING THEORY}

We can then decompose $\overline{\mathcal{D}}_{\alpha \beta}$ into a symmetric and anti-symmetric part, to see what the fermion bispinor will be after we have performed a T-duality on the superspace.

If we wan to work only with the RR-fluxes it can be more convenient to find how these RR-fluxes directly transform under a T-duality. This was originally done in [190].

Because the different RR-fluxes have to be combined with gamma matrices to form the fermion bispinor, the gamma matrices on the target space 'determine' which parts of the fermion bispinor $\mathbb{F}_{\alpha \beta}$ will be associated with the different RR-fluxes. Under a T-duality (on one of the bosonic directions of the spacetime manifold) the bosonic metric and $B_{\mu \nu}$-field will change according to the Buscher rules, and the gamma matrices on the bosonic target space will change as well. If we can therefore figure out how the gamma matrices transform under a T-duality, we can use the transformation of these gamma-matrices to obtain the RR-fluxs after the T-duality.

As we have seen in eq.(2.118) the Maurer-Cartan forms $J_{+}$and $J_{-}$(equivalent to the vielbeins) of the isometry direction transform differently under a T-duality. These two vielbeins $J_{ \pm}$describe the same target space, and are related by a Lorentz transformation

$$
J_{+}=\Lambda J_{-} .
$$

We can then find a spinor representation corresponding to this Lorentz transformation $\Omega$, that will show us how the target space gamma matrices will transform

$$
\Omega^{-1} \Gamma^{i} \Omega=\Lambda_{j}^{i} \Gamma^{j}
$$

Once we obtained $\Omega$, we can then use it directly to see how the fermion bispinor transforms when we perform a T-duality on one of the bosonic directions of the original target space

$$
e^{\bar{\Phi} / 2 \bar{F}^{\alpha \beta}}=e^{\Phi / 2} \not{F}^{\alpha \beta} \Omega^{-1} .
$$

We will show an explicit example of this when we discuss the non-Abelian T-duality in section 2.9.1.

\subsubsection{T-Duality and Integrability}

If we start from an integrable string background (for example a coset space, so that we can write a Lax connection following the ideas in section 2.2.1), the integrable structure of the string worldsheet will be preserved under a T-duality. This can most easily be seen by thinking of T-duality as a canonical transformation, following [83].

In the original sigma model of eq.(2.110) we have a canonical position and momentum for the $\theta$-direction

$$
q^{\theta}=X^{\theta} \quad p_{a}^{\theta}=\frac{\partial \mathcal{L}}{\partial \dot{q}_{a}^{\theta}}=m^{a b} D_{\mu \theta} \partial_{b} X^{\mu}+k^{a b} \partial_{b} X^{\theta}
$$


where we use the abbreviation $m^{a b} D_{\mu \nu}=\eta^{a b} G_{\mu \theta}+\epsilon^{a b} B_{\mu \theta}$, and $k=G_{\theta \theta}$.

If we look how the T-duality acts on $J_{a}=\partial_{a} X^{\theta}$ in eq.(2.118) we see, using eq.(2.116), that the derivative $\dot{q}_{a}^{\theta}$ is mapped after the T-duality to minus the momentum of the worldsheet on the dual space

$$
\begin{aligned}
\dot{q}_{a}^{\theta}=\partial_{a} X^{\theta} \rightarrow & k^{-1}\left[m^{a b} D_{\theta \rho} \partial_{b} X^{\rho}-\epsilon^{a b} \partial_{b} \bar{\theta}\right]=\left[m^{a b} \bar{D}_{\theta \rho} \partial_{b} X^{\rho}-\epsilon^{a b} \partial_{b} \bar{\theta}\right] \\
= & \frac{\partial \mathcal{L}_{\text {T-dual }}}{\partial \dot{\bar{q}}_{a}^{\theta}}=-\bar{p}_{b}^{\bar{\theta}} .
\end{aligned}
$$

The T-duality thus maps $\dot{q}_{a}^{\theta} \rightarrow-\bar{p}_{a}^{\bar{\theta}}$. Similarly we can find that the momentum on the worldsheet on the initial space is mapped to $p_{a}^{\theta} \rightarrow-\dot{\bar{q}}_{a}^{\bar{\theta}}$.

We can therefore think of the T-duality as a canonical transformation that acts on the phase space variables (the worldsheet position $q_{a}^{\theta}$ and momentum $p_{a}^{\theta}$ along the $\theta$-direction), that preserves the symplectic structure of the phase space, and maps them to a different position $q_{a}^{\bar{\theta}}$ and momentum $p_{a}^{\bar{\theta}}$ that describe the fluctuations of the worldsheet along the $\bar{\theta}$-direction of the dual space [83].

$$
\left(\begin{array}{ll}
\dot{q}_{a}^{\theta}, & p_{a}^{\theta}
\end{array}\right) \quad \rightarrow \quad\left(-\bar{p}_{a}^{\theta}, \quad-\dot{\bar{q}}_{a}^{\theta}\right)
$$

\subsection{Non-Abelian T-duality}

Non-Abelian T-duality is a generalisation of the (Abelian) T-duality we introduced in the previous sections, where we now consider a target spacetime with a subspace that is equivalent to a non-Abelian Lie group $G$. This work started with [32], where the T-duality transformations for the NS-sector of string theory were generalised to the case of non-Abelian isometries. Later in [190], these transformations were extended by incorporating the transformation of the RR-fields, providing a method to generate new solutions in type II supergravity, starting from backgrounds with non-Abelian isometries.

As we did for the Abelian case in eq.(2.109), we start by decomposing the metric and $B$-field into a part on the non-Abelian symmetry group $G$, and write

$$
\begin{gathered}
G_{\mu \nu} d x^{\mu} d x^{\nu}=G_{\rho \sigma} d x^{\rho} d x^{\sigma}+2 G_{\rho i} d x^{\rho} J^{i}+g_{i j} J^{i} J^{j} \\
B_{\mu \nu} d x^{\mu} \wedge d x^{\nu}=B_{\rho \sigma} d x^{\rho} \wedge d x^{\sigma}+B_{\rho i} d x^{\rho} \wedge J^{i}+\frac{1}{2} b_{i j} J^{i} \wedge J^{j}
\end{gathered}
$$

where $J_{a}^{A}=e_{\mu}^{A} \partial_{a} X^{\mu}=g^{-1} \mathrm{~d} g$ (as in section 2.2) is the Maurer-Cartan form on the nonAbelian symmetry group $G$. The procedure for the non-Abelian T-duality now carries through analoguous to the Abelian case in section 2.8.1:

- We start again by writing the action for the sigma model on the above target space (following eq.(2.111)), and promote the isometry group on which we want to perform the 


\section{INTEGRABILITY AND SUPERSTRING THEORY}

T-duality to a local gauge symmetry by replacing the derivatives with gauge covariant derivatives $J_{a}^{i}=\partial_{a} \theta^{i} \rightarrow D_{a} \theta^{i}=\partial_{a} \theta^{i}+A_{a}^{i}$. Note this now requires the introdution of a non-Abelian gauge field $A_{a}=A_{a}^{i} \tau_{i}$, with $\tau_{i}$ the generators of $G$.

- Following eq.(2.112) we add a Lagrange multiplier term to the action, that forces $A_{a}^{A}$ to be pure gauge and have vanishing field strength. Since $A_{a}^{i}$ is non-Abelian the Lagrange multiplier now has to be of the form $\operatorname{Tr}\left[\bar{\theta}^{i} F_{a b}^{j}\right]$ where the Lagrange multipier field $\bar{\theta}^{i}$ also has an index on the algebra of $G$. The equation of motion for $\bar{\theta}^{i}$ will then be $F_{\mu \nu}^{i}=0$, or equivalently $D_{a} J_{b}^{i}=\partial_{a} J_{b}^{i}-\left[J_{a}^{j}, J_{b}^{k}\right]=0$. After partial integration we can rewrite the Lagrange multiplier term to read

$$
\operatorname{Tr}\left[\bar{\theta}^{i} F_{a b}^{j}\right]=\operatorname{Tr}\left[\bar{\theta}^{i} \partial_{a} A_{b}^{j}-\bar{\theta}^{j} \partial_{b} A_{a}^{i}-\bar{\theta}^{k}\left[A_{a}^{i}, A_{b}^{j}\right]\right]=\operatorname{Tr}\left[\partial_{b} \bar{\theta}^{j} A_{a}^{i}-\partial_{a} \bar{\theta}^{i} A_{b}^{j}-A_{a}^{j} f A_{b}^{k}\right],
$$

where $f=f_{i j}{ }^{k} \bar{\theta}_{k}$.

We can now write the partition function as

$$
\begin{aligned}
& Z=\int \mathcal{D} X^{\rho} \mathcal{D} A_{a}^{i} \mathcal{D} \bar{\theta}^{i} e^{-i S\left[X^{\rho}, V_{a}, \bar{\theta}\right]}, \quad \text { with } \\
& S=\frac{1}{2 \pi \alpha^{\prime}} \int_{\Sigma} d^{2} z \operatorname{Tr} A_{a}^{i}\left(\eta_{i j}+f\right) A_{b}^{j}+2\left(\gamma^{a b} G_{\theta \rho} \partial_{a} X^{\rho}+\epsilon^{a b} B_{\theta \rho} \partial_{a} X^{\rho}-\epsilon_{i j}^{a b} \tilde{\theta}^{i} \partial_{a}\right) A_{b}^{j}+ \\
& +\gamma^{a b} G_{\rho \sigma} \partial_{a} X^{\rho} \partial_{b} X^{\sigma}+B_{\rho \sigma} \partial_{a} X^{\rho} \partial_{b} X^{\sigma}+\alpha^{\prime} \Phi R
\end{aligned}
$$

Where we fixed the gauge $A_{a}^{i}=J_{a}^{i}$, and $\eta_{i j}$ and $f_{i j}{ }^{k}$ are respectively the Killing form and structure constants on $G$. If we define the matrix

$$
M_{i j}=\eta_{i j}+f_{i j}^{k} \theta_{k}
$$

and integrate out $A_{a}^{i}$, we obtain a new sigma model on a target space $\bar{G}_{\mu \nu}, \bar{B}_{\mu \nu}, \bar{\Phi}$. This then gives us a generalisation of the Buscher rules for the non-Abelian case.

$$
\begin{aligned}
\bar{D}_{\mu \nu} & =\left(\begin{array}{c|c}
D_{\mu \nu}-D_{\mu i} M_{i j}^{-1} D_{j \nu} & -D_{\mu i} M_{i j}^{-1} \\
\hline M_{i j}^{-1} D_{j \nu} & M_{i j}^{-1}
\end{array}\right) \\
\bar{\Phi} & =\Phi-\frac{1}{2} \ln (\operatorname{det} M)
\end{aligned}
$$

where as before $D_{\mu \nu}=G_{\mu \nu}+B_{\mu \nu}$ and we absorbed the factor $(\operatorname{det} M)^{-\frac{1}{2}}$ that appears when integrating out $A_{a}^{i}$ into a change of the dilaton. 


\subsubsection{Example: NATD on SU(2)}

As an example we will here illustrate the NATD procedure on a superspace that is described by a bosonic metric for $S^{3} \cong S U(2)$, together with a dilaton $\Phi_{0}$ and RR-flux $F_{3}=f$ vol $\Omega_{3}$.

The metric and vielbeins will in that case be those described in section 2.2. The matrix $M_{i j}$ can be constructed from the Killing form $\eta_{i j}$ and the structure constants $f_{i j}^{k} \theta_{k}$ on the group. In the case of $S U(2)$ these are given by

$$
\eta_{i j}=\delta_{i j}, \quad \text { and } \quad f_{i j}{ }^{k} \theta_{k}=\epsilon_{i j}{ }^{k} \theta_{k} .
$$

Note the $\theta^{i}$ here indicate the different (bosonic) Lagrange multiplier fields (and should not be confused with fermionic variables). The above expressions then give us

$$
M_{i j}=\delta_{i j}+f_{i j}{ }^{k} \theta_{k}=\left(\begin{array}{ccc}
1 & \theta_{3} & -\theta_{2} \\
-\theta_{3} & 1 & \theta_{1} \\
\theta_{2} & -\theta_{1} & 1
\end{array}\right)
$$

from where we find the inverse matrix

$$
M_{i j}^{-1}=\left(\begin{array}{ccc}
\frac{\left(\theta_{1}\right)^{2}+1}{\rho^{2}+1} & \frac{\theta_{1} \theta_{2}-\theta_{3}}{\rho^{2}+1} & \frac{\theta_{1} \theta_{3}+\theta_{2}}{\rho^{2}+1} \\
\frac{\theta_{1} \theta_{2}+\theta_{3}}{\rho^{2}+1} & \frac{\left(\theta_{2}\right)^{2}+1}{\rho^{2}+1} & \frac{\theta_{2} \theta_{3}-\theta_{1}}{\rho^{2}+1} \\
\frac{\theta_{1} \theta_{3}-\theta_{2}}{\rho^{2}+1} & \frac{\theta_{2} \theta_{3}+\theta_{1}}{\rho^{2}+1} & \frac{\left(\theta_{3}\right)^{2}+1}{\rho^{2}+1}
\end{array}\right)
$$

where $\rho^{2}=\left(\theta_{1}\right)^{2}+\left(\theta_{2}\right)^{2}+\left(\theta_{3}\right)^{2}$. By separating the symmetric and anti-symmetric components in the above matrix we find the metric and $B_{2}$-field on the dual target space, which are

$$
\begin{aligned}
d s^{2} & =\frac{\theta_{i} \theta_{j}+\delta^{i j}}{1+\rho^{2}} d \theta_{i} d \theta_{j} \\
B_{2} & =\frac{\epsilon^{i j k} \theta_{i}}{1+\rho^{2}} d \theta_{j} \wedge d \theta_{k}
\end{aligned}
$$

It is more convenient to choose a different set of coordinates to describe this dual space. We choose $\theta_{1}=\rho \sin \chi \cos \xi, \theta_{2}=\rho \sin \chi \sin \xi, \theta_{3}=\rho \cos \chi$, with $\rho>0$. In terms of which the dual metric and $B$-field read

$$
\begin{aligned}
d s^{2} & =d \rho^{2}+\frac{\rho^{2}}{1+\rho^{2}} d \Omega_{2}^{2} \\
B_{2} & =\frac{\rho^{3}}{1+\rho^{2}} d \Omega_{2}
\end{aligned}
$$

The dilaton on the new space is given by

$$
e^{\Phi}=\frac{e^{\Phi_{0}}}{\sqrt{1+\rho^{2}}}
$$




\section{INTEGRABILITY AND SUPERSTRING THEORY}

As an example of how the RR-fluxes transform under NATD, we will show this here in detail. As an example we consider an $F_{3}$ flux on the original space, of the form

$$
F_{3}=f \operatorname{vol} \Omega_{3}=F_{3}=f e_{1} \wedge e_{2} \wedge e_{3},
$$

To form the fermion bispinor we first replace the wedged frame fields $e^{a} \wedge e^{b} \ldots$ by the corresponding anti-symmetrised gamma matrices $\Gamma^{a b \cdots}$.

$$
\text { 俄 }=f \Gamma^{123}
$$

The $\Omega$-matrix for the NATD on $\mathrm{SU}(2)$ is given by [84]

$$
\Omega=\frac{\Gamma_{11}}{\sqrt{1+\rho^{2}}}\left(\Gamma^{123}+\theta^{i} \Gamma^{i}\right)
$$

Under the NATD the RR-flux then transforms - as we explained in section 2.8.2 - as

$$
\begin{aligned}
e^{\Phi} & =e^{\Phi_{0}}{ }_{f} \Omega^{-1} \\
& =\frac{e^{\Phi_{0}} f}{\sqrt{1+\rho^{2}}}\left(1+\epsilon^{i j k} \theta^{i} \Gamma^{j k}\right)
\end{aligned}
$$

Using that $e^{\Phi}=e^{\Phi_{0}}\left(1+\rho^{2}\right)^{-\frac{1}{2}}$, this then gives us for the fermion bispinor on the dual space

$$
\overline{\text { F }}=f\left(1+\epsilon^{i j k} \theta^{i} \Gamma^{j k}\right) \text {. }
$$

To find the final expressions for the RR-fluxes on the dual targetspace we use that in the coordinates $(\rho, \chi, \xi)$ that we defined earlier $\theta_{1} e_{2} \wedge e_{3}+\theta_{2} e_{3} \wedge e_{1}+\theta_{3} e_{1} \wedge e_{2}=\rho e_{\chi} \wedge e_{\xi}$.

Together with the fact that $e_{\chi} \wedge e_{\xi}=\frac{\rho^{2}}{1+\rho^{2}} d \Omega_{2}$ this then gives us for the RR-fluxes on the dual space

$$
F_{0}=f, \quad F_{2}=f \frac{\rho^{3}}{1+\rho^{2}} \text { vol } \Omega_{2}
$$

\subsection{T-duality at the Quantum Level}

The Abelian T-duality between the full partition functions in eqs.(2.113) and (2.115) holds at the quantum level. To see this in more detail, we will here examine that the partition function following from the action with the Lagrange multiplier in eq.(2.112) is indeed equivalent, after integrating out the gauge field $A_{a}$, to the partition function for the original action in eq.(2.111).

As we have seen in section 2.8.1, the equations of motion for $\bar{\theta}$ imply that $A_{a}$ is a flat connection. Integrating out $A_{a}$ therefore causes the Lagrange multiplier term to vanish

$$
S_{L M}=\frac{1}{2 \pi \alpha^{\prime}} \int_{\Sigma} d z^{2} \bar{\theta} \epsilon^{a b} \partial_{a} A_{b}
$$


However, there can be non-trivial windings (or holonomies) of the gauge field around different directions on the worldsheet. For a worldsheet of genus $g$ there are $2 g$ such non-trivial holonomies. When we integrate out $A_{a}$ we also have to integrate over all of these configurations with non-trivial holonomies.

In order to do this, we first decompose the field $A_{a}$ as $A_{a}=A_{a}^{0}+\tilde{A}_{a}$, where $A_{a}^{(0)}$ contains fluctuations of $A_{0}$ with trivial holonomies, and $\tilde{A}_{a}$ containing the configurations with different non-trivial windings. Using this decomposition the Lagrange multiplier term can be written as

$$
S_{L M}=\frac{1}{2 \pi} \int_{\Sigma} d z^{2} \bar{\theta}\left(\partial_{a} A_{b}^{0}-\partial_{b} A_{a}^{0}\right)+\frac{1}{2 \pi} \sum_{i=1}^{g}\left(n_{a_{i}} \oint_{b_{i}} \tilde{A}_{b}-n_{b_{i}} \oint_{a_{i}} \tilde{A}_{a}\right)
$$

where the $n_{a_{i}}=\oint_{a_{i}} d \bar{\theta}$ are the winding numbers around the different cycles on the worldsheet.

Integrating out $A_{a}^{0}$ can be done as before, but when we integrate out $\tilde{A}_{a}$, the winding sector will give a contribution to the partition function of the form

$$
\sum_{i}^{g} e^{\frac{1}{2 \pi}\left(n_{a_{i}} \oint_{b_{i}} \tilde{A}_{a}-n_{b_{i}} \oint_{a_{i}} \tilde{A}_{b}\right)}=\sum_{m, n=1}^{g} \delta\left(m-\frac{n_{a_{i}}}{2 \pi} \oint_{b_{i}} \tilde{A}_{b}\right) \delta\left(n-\frac{n_{b_{i}}}{2 \pi} \oint_{a_{i}} \tilde{A}_{b}\right) .
$$

If $\bar{\theta}$ has a periodicity of $2 \pi$ the winding numbers $n_{a_{i}}$ and $n_{b_{i}}$ will be $2 \pi$ times an integer number, and the above summation simply becomes a sum over identity elements of $U(1)$. We then find that adding the Lagrange multiplier does not change the partition function, and the Abelian T-duality holds even at the quantum level for the worldsheet theory.

For the non-Abelian T-duality this equivalence no longer holds at the quantum level. This can be seen by going over the above arguments, and considering a Lagrange multiplier term for a non-Abelian gauge field $A_{a}^{i}$ as we have in eq.(2.130).

$$
S_{L M}=\frac{1}{2 \pi \alpha^{\prime}} \int_{\Sigma} d z^{2} \bar{\theta} \epsilon^{a b} \partial_{a} A_{b} \operatorname{Tr}\left[\bar{\theta}^{i} F_{a b}^{j}\right]
$$

We can again split $A_{a}^{i}=A_{a}^{(0) i}+\tilde{A}_{a}^{i}$ and integrate out $A_{a}^{(0) i}$. The contributions of the nontrivial holonomies of $\tilde{A}_{a}^{i}$ around the different cycles of the string worldsheet will now involve (path-ordered) holonomies of the non-Abelan gauge field. These cannot be integrated out as nicely as in the Abelian case. It is therefore believed that the non-Abelian T-duality is not a 'true' equivalence between the different partition functions for the string on the backgrounds obtained before and after a NATD [51, 53].

It is however a classical equivalence between the different sigma models. It maps us from one (conformal) sigma model to another, and as a result the target space obtained after a NATD will still be a supergravity solution. Since Non-Abelian T-duality breaks the symmetries on which on performs the duality, it can be used as a 'solution generating technique'. Starting from 


\section{INTEGRABILITY AND SUPERSTRING THEORY}

a very symmetric solution one can perform a NATD, break various symmetries, and obtain less symmetric supergravity solutions. Because (non-)Abelian T-dualities preserve integrability, this has the additional benefit that if we start from a background on which the superstring is integrable, this will still be the case on the resulting less symmetric dual background. We will

illustrate the power of NATD as a solution generating technique in more detail in chapter 7 
III. ADS/CFT AND INTEGRABILITY FOR HALF-SUPERSYMMETRIC SCFTS

\section{Chapter 3}

\section{AdS/CFT and Integrability for Half-Supersymmetric SCFTs}

\subsection{Introduction}

In this chapter we will first introduce branes in string theory and supergravity, in section 3.2. We will then explain how they are related to the AdS/CFT correspondence in section 3.3. The AdS/CFT corresondence relates the states of a conformal field theory (CFT) to strings propagating on an Anti-de Sitter (AdS) spacetime. In the original (and now most famous and well studied) example [3] this concernes the relation between strings on $\mathrm{AdS}_{5} \times \mathrm{S}^{5}$ - a supergravity solution that arises as the near horizon geometry of a stack of $N$ D3-branes and a four-dimensional superconformal field theory (SCFT) known as $\mathcal{N}=4$ supersymmetric Yang-Mills (SYM) theory. We will briefly sketch how the ideas of integrability apply to the AdS/CFT correspondence in section 3.4.

In section 3.5 we will then introduce more complicated $\mathrm{D}_{p}-\mathrm{D}_{p+2}$-NS5 brane set-ups. The near-horizon geometries of these brane configurations are thought to be half-supersymmetric $\mathrm{AdS}_{p+1}$ supergravity solutions, that we will here introduce for different dimensions $p=6,5,4,3$ in section 3.6. Following the AdS/CFT correspondence, these spaces will then be dual to half-supersymmetric $p$-dimensional SCFTs with bifundamental hypermultiplets, and a quiver structure for the colour and flavour groups. We give a review of this for different dimensions, and explain what is known regarding the integrability of strings on these supergravity backgrounds.

In the following chapters of this thesis we will focus in more detail on the D6-D8-NS5 set-ups that allow us to study $6 \mathrm{~d} \mathcal{N}=(1,0)$ SCFTs. 


\section{$3.2 \quad$ D-Branes in String Theory}

In the previous chapter we have only concerned ourselves with the dynamics of closed strings. In addition one can consider open strings, the endpoints of which are located on higher dimensional planes called $\mathrm{D}_{p}$-branes (that have a $p+1$ dimensional worldvolume, consisting of $p$ spatial directions and one time direction) [64]. These $D_{p}$-branes turn out to be sources of the RR-fluxes [63] that we introduced earlier in the context of superspaces in section 2.5.

These $\mathrm{D}_{p}$-branes are allowed to fluctuate, but their fluctuations have to be such that the worldsheet of the open string ending on them remains conformal. One can show that this condition implies the fluctuations of these branes then have to be described by the Dirac-BornInfeld action [66, 67]

$$
S_{\text {brane }}=S_{D B I}+S_{W Z}+S_{\text {fermions }} .
$$

where

$$
\begin{gathered}
S_{D B I}=-T_{p} \int_{\mathcal{M}_{p}+1} d^{p+1} \sigma e^{-\Phi} \sqrt{-\operatorname{det}\left(g_{a b}-\mathcal{F}_{a b}\right)} \\
S_{W Z}=T_{p} \int_{\mathcal{M}_{p}+1} \mathcal{C} \wedge e^{-\mathcal{F}}
\end{gathered}
$$

Here $\mathcal{F}_{a b}=B_{a b}-\frac{\alpha^{\prime}}{2 \pi} F_{a b}$, with $F_{a b}$ the field strength of an additional Born-Infeld U(1) gauge field that lives on the worldvolume of the brane, while $\Phi, g_{a b}$ and $B_{a b}$ are the pullbacks of these fields to the worldvolume of the brane. This DBI part of the action describes the 'kinetic' part of the brane, and how it couples to these fields in the NS-NS sector. Its dynamics are such that the brane (just like the string) wants to minimise its surface area, while taking into account the background fields that it couples to.

Analogous to the strings, the $\mathrm{D}_{p}$-branes have a tension of their own that is related to the string tension $T_{s}=\left(2 \pi \alpha^{\prime}\right)^{-1}$. We can combine the factors of $T_{p}$ and $e^{-\Phi}=g_{s}^{-1}$ into a D-brane tension (mass per unit surface or volume area) that we now see is inversely proprtional to the string coupling $g_{s}[52]$

$$
\mathcal{T}_{p}=\frac{T_{p}}{e^{\Phi}}=(2 \pi)^{\frac{1}{2}(1-p)} \frac{T_{s}^{\frac{1}{2}(p+1)}}{g_{s}} \sim g_{s}^{-1} .
$$

Since the tension of the $\mathrm{D}_{p}$-branes is inversely proportional to the string coupling $g_{s}$, in the semi-classical limit of the string where both $g_{s} \rightarrow 0$ and $\alpha^{\prime} \rightarrow 0$, the D-branes are very heavy non-perturbative objects with only small fluctuations. As we turn on the string coupling $g_{s}$, the tension of the $\mathrm{D}_{p}$-branes goes down and their fluctuations become increasingly wilder.

The $S_{W Z}$ term in the action of eq.(3.1) describes the coupling of the brane to the R-R fields. Here $\mathcal{C}=\sum_{r=0}^{p} C_{r}$ is a sum over all R-R gauge potentials $F_{p+1}=d C_{p}$. The wedge product with the exponential of $\mathcal{F}$ can be expanded as series of wedge products, so that this term (in the absence of a Born-Infeld field) is of the form

$$
\mathcal{C} \wedge e^{-\mathcal{F}}=C_{p}-C_{p-2} \wedge B_{2}+\frac{1}{2} C_{p-4} \wedge B_{2} \wedge B_{2}-\frac{1}{6} C_{p-6} \wedge B_{2} \wedge B_{2} \wedge B_{2}+\ldots
$$




\section{ADS/CFT AND INTEGRABILITY FOR HALF-SUPERSYMMETRIC SCFTS}

\subsubsection{Worldvolume Theories of D-Branes}

We will now examine what the fluctuations of a D-brane look like in the $\alpha^{\prime} \rightarrow 0$ limit. We do this by expanding the DBI action for the D-brane on a flat background (setting all of the sources, except the $U(1)$ field $F_{a b}$ on the worldsheet to zero) in powers of $\alpha^{\prime}$ and find an effective action of the form

$$
S_{e f f}=\frac{1}{g_{Y M}^{2}} \int d^{p+1} x \frac{1}{4} F_{a b} F^{a b}+\partial_{a} X^{\mu} \partial^{a} X_{\mu}+\text { fermions. }
$$

This action is equivalent to a $p+1$-dimensional supersymmetric Yang-Mills theory, where the Yang-Mills coupling is now given by

$$
g_{Y M}^{2}=\frac{(2 \pi)^{p-2} g_{s}}{\alpha^{\prime \frac{1}{2}(3-p)}} .
$$

In this worldvolume theory, one can interpret the scalar fields $X^{\mu}$ as describing the transverse fluctuations of the brane. The points where strings end on the brane look like particles that are charged under the Born-Infeld field $F_{a b}$ on the brane's worldvolume.

We can now generalise this to a stack of $N$ parallel coincident $\mathrm{D}_{p}$-branes. Strings can now have one of their endpoints on one of these $N$ different branes in the stack, and their other endpoint on another brane, giving $N^{2}$ possibilities. Since the endpoints of the string are associated with particles in the worldvolume theory, we now have $N^{2}$ different particles. We can organise these different scalars $\left(X^{\mu}\right)_{j}^{i}$ and fermions into an $N \times N$ matrix that now both transform under in the adjoint representation of $U(N)$. Similarly the $F_{a b}$ gauge field becomes a non-Abelian $U(N)$ gauge field [65].

This gauge group $U(N)=S U(N) \times U(1)$ splits into a $U(1)$ that decouples and describes the degrees of freedom for the center of mass of the brane stack (and is often ignored in the literature). The remaining $S U(N)$ gauge group describes the fluctuations of the different branes around the center of mass. ${ }^{1}$ Since the scalar fields $X^{\mu}$ are now non-Abelian, the DBI action for a stack of coincident $\mathrm{D}_{p}$-branes will also contain additional terms involving their commutators.

As before we can expand in $\alpha^{\prime}$, which will now give an effective action that is a non-Abelian supersymmetric Yang-Mills theory

$$
S_{\text {eff }}=\frac{1}{g_{Y M}^{2}} \int d^{p+1} x \operatorname{Tr}\left[\frac{1}{4} F_{a b} F^{a b}+\mathcal{D}_{a} X^{\mu} \mathcal{D}^{a} X_{\mu}+\mathcal{O}([X, X])+\text { fermions }\right]
$$

where $\mathcal{D}_{a} X^{\mu}=\partial_{a} X^{\mu}-\left[A_{a}, X^{\mu}\right]$.

\footnotetext{
${ }^{1}$ For this reason we will often be talking about a D3-brane with an $S U(N)$ gauge group.
} 


\subsubsection{P-Branes in Supergravity}

The D-branes we have discussed so far also show up as solutions of the supergravity equations that we introduced in section 3.5.2. Since the branes are incredibly heavy in the $g_{s} \rightarrow 0$ limit, they will look like $p$-dimensional generalisations of black hole like solutions that are also called (black) p-branes. Just like a black hole can be electromagnetically charged, these p-branes can be charged under the antisymmetric (Ramond-Ramond) form $F_{p+1}=d C_{p}$ that we introduced earlier in section 2.5 .

In the string frame the metric, dilaton and RR-flux for these extremal $p$-brane solutions is given by

$$
\begin{aligned}
d s_{p}^{2} & =H_{p}^{-\frac{1}{2}}(r) \eta_{\mu \nu} d x_{\|}^{\mu} d x_{\|}^{\nu}+H_{p}^{\frac{1}{2}}(r)\left(d r^{2}+r^{2} d \Omega_{8-p}^{2}\right) \\
e^{\Phi} & =H_{p}^{-\frac{1}{4}(p-3)}, \\
F_{p+1} & =d C_{p}+\star d C_{p}, \quad C_{p}=-\frac{1}{2}\left(H_{p}^{-1}(r)-1\right) e^{0} \wedge e^{1} \wedge \ldots \wedge e^{p},
\end{aligned}
$$

where $H_{p}(r)$ is a harmonic form

$$
H_{p}(r)=\left(1+\frac{2 c_{p} Q_{p}}{r^{7-p}}\right)
$$

with $c_{p}$ a constant and $Q_{p}$ the charge of the brane.

It was in a seminal paper that Polchinski [63] showed the D-branes on which open strings can end are indeed identical to these $p$-brane solutions in supergravity.

Similar to normal black hole solutions, near-extremal $p$-brane supergravity solutions have an horizon and emit Hawking radiation. In terms of string theory, this Hawking radiation can now be understood as open strings ending on the brane, that can split off to form a closed string that can move away from the brane.

\subsection{The AdS/CFT Correspondence}

The AdS/CFT correspondence follows from examining the process of open strings that can split off from the D-brane in more detail. This relation was originally proposed by Maldacena in [3], subsequently developed in [69, 70], and reviewed in [71], where the authors illustrated this in detail for a stack $N$ coinciding D3-branes.

The worldvolume theory for a stack of $N$ coinciding D3-branes is given by a DBI action and WZ-term as we explained in section 3.2. In order to understand the process by which open strings can split off from the brane, we have to consider the full DBI and WZ actions 


\section{ADS/CFT AND INTEGRABILITY FOR HALF-SUPERSYMMETRIC SCFTS}

$S_{\text {brane }}=S_{D B I}+S_{W Z}$ for the stack of D3-branes, the action $S_{\text {bulk }}$ for the closed strings that move on and backreact with the spacetime surrounding the D3-branes, together with additional interactions between these two systems.

$$
S=S_{\text {brane }}+S_{\text {bulk }}+S_{\text {interactions }}
$$

We will now consider this in the $\alpha^{\prime} \rightarrow 0$ limit. The worldvolume theory on the stack of D3branes reduces in this case to $4 \mathrm{~d}$ supersymetric Yang-Mills theory, with $\mathcal{N}=4$ supersymmetry and an $S U(N)$ gauge group (see section 3.2.1). The action for the open strings far away from the braens reduces in the $\alpha^{\prime} \rightarrow 0$ limit to the supergravity equations of motion (see section 2.7) on the flat bulk-spacetime. The interaction term between the open and closed strings consists in this limit of the leading order terms from the DBI action that couple the fluctuations of the brane to the background fields. It turns out that this interaction term vanishes in the $\alpha^{\prime} \rightarrow 0$ limit so that the action for the resulting system reduces to two decoupled systems [65]

$$
S=S_{S Y M}+S_{\text {free open strings }} .
$$

\subsubsection{Open Strings on $\operatorname{AdS}_{5} \times \mathbf{S}^{5}$}

Let us now consider the same system from the supergravity point of view. In section 3.2 .2 we have seen that the spacetime around a brane is curved, and is given by the $p$-brane supergravity solutions of eq.(3.8). Around a stack of D3-branes this geometry is of the form

$$
d s^{2}=\left(1+\frac{L^{4}}{r^{4}}\right)^{-\frac{1}{2}} \eta_{\mu \nu} d x^{\mu} d x^{\nu}+\left(1+\frac{L^{4}}{r^{4}}\right)^{\frac{1}{2}}\left(d r^{2}+r^{2} d \Omega_{5}^{2}\right),
$$

with $L^{4}=4 \pi g_{s} N \alpha^{\prime 2}$. The dilaton (or string coupling) is a constant $e^{\Phi}=g_{s}$ for this geometry surrounding the D3-brane. In addition there will be an $F_{5}$ RR-flux that we will omit for the moment.

We see that in the $\alpha^{\prime} \rightarrow 0$ limit, the action describing the worldvolume of the brane backreating on the surrounding spacetime, splits into a part that describes the low energy fluctuations of the brane, and a part that describes open strings far away from the branes that no longer interact with it. We can now consider these two different regimes also in terms of open strings moving on this supergravity background. Far away from the D3-branes, for $r \gg L$ we see that $\left(1+\frac{L^{4}}{r^{4}}\right) \sim 1$ and the geometry looks like flat spacetime. The open strings propagating here have enough energy to climb out of the gravitational well surrounding the D3-branes, and will no longer interact with it. This is identical to the system described by $S_{\text {free open string }}$ in eq.(3.11).

The low energy strings will not be able to get out of this gravitational well, and will remain trapped at $r \ll L$. Here the geometry is curved and $\left(1+\frac{L^{4}}{r^{4}}\right) \sim \frac{L^{4}}{r^{4}}$. This limit is also known 
as the near-horizon limit or the throat of the geometry. Since the open strings inside the nearhorizon will not be able to leave we find again two decoupled systems from the supergravity point of view:

$$
S=S_{\text {near-horizon }}+S_{\text {free open strings }} .
$$

Open strings that move in the near-horizon limit of the spacetime and open strings far away from the brane that only see a flat target space.

If we now compare this with eq.(3.11) we can identify both of the low energy systems in these two different points of view. The low energy fluctuations of the brane described by the supersymmetric Yang-Mills action $S_{S Y M}$ should somehow also describe open strings moving on the near-horizon geometry of the branes. This is the essence behind the AdS/CFT correspondence; that the SYM theory describing the fluctuations of the D3-branes has to be equivalent to closed strings moving on the near-horizon geometry of $p$-brane supergravity solution

$$
S_{S Y M}=S_{\text {near-horizon }}
$$

To study the near horizon geometry in more detail we first define a new radial coordinate $u=L^{2} / r$

$$
d s^{2}=\left(1+\frac{L^{4}}{u^{4}}\right)^{-\frac{1}{2}} \frac{L^{2}}{u^{2}} \eta_{\mu \nu} d x^{\mu} d x^{\nu}+\left(1+\frac{L^{4}}{u^{4}}\right)^{\frac{1}{2}} L^{2}\left(\frac{d u^{2}}{u^{2}}+r^{2} d \Omega_{5}^{2}\right)
$$

The $r \rightarrow 0$ limit now corresponds to $u \rightarrow \infty$. In this limit the geometry looks like

$$
d s^{2}=\frac{L^{2}}{u^{2}}\left(d u^{2}+\eta_{\mu \nu} d x^{\mu} d x^{\nu}\right)+L^{2} d \Omega_{5}^{2},
$$

which is the Poincaré patch of $\operatorname{AdS}_{5} \times \mathrm{S}^{5}$, where $L^{2}$ is the radius of both the $\mathrm{AdS}_{5}$ and $\mathrm{S}^{5}$ spaces. If we consider the Polyakov action of eq.(2.14) on this geometry, the string tension now combines with the radius $L$ to an overall prefactor of

$$
T_{s} L^{2}=\frac{L^{2}}{4 \pi \alpha^{\prime}}=\sqrt{\frac{g_{s} N}{4 \pi}}=\sqrt{\frac{\lambda}{4 \pi}},
$$

where we introduced $\lambda=g_{s} N$. When we now take the $\alpha^{\prime} \rightarrow 0$ limit, the ratio $L^{2} / \alpha^{\prime}=\lambda$ stays fixed. We can trust this supergravity description for the open strings as long as $\alpha^{\prime} \ll L$.

The near horizon $\mathrm{AdS}_{5} \times \mathrm{S}^{5}$ geometry of a stack of D3-branes thus correspond to the vacuum of $\mathcal{N}=4$ worldvolume theory on the branes, while supergravity excitations of this geometry corresponding to excitations of the SYM theory. 


\section{ADS/CFT AND INTEGRABILITY FOR HALF-SUPERSYMMETRIC SCFTS}

\subsection{2 $\mathcal{N}=4$ Super Yang-Mills}

Let us now examine in more detail $S_{S Y M}$ for the open strings that end on the stack of D3branes. We have seen in section 3.2.1 that the fluctuations of the branes are described in the $\alpha^{\prime} \rightarrow 0$ limit by a supersymmetric Yang Mills theory.

For a stack of D3-branes this worldvolume theory will be $\mathcal{N}=4$ super-Yang-Mills (SYM). The fields of this theory are six scalars $\Phi^{i}$, a gauge field $A_{\mu}$ with a corresponding field strength $F_{\mu \nu}$, and fermions $\Psi_{\alpha}^{a}, \bar{\Psi}_{\dot{\alpha} a}$ that fill the $\mathcal{N}=4$ supersymmetry multiplet. The bosonic part of the Lagrangian for this theory is of the form

$$
S_{S Y M}=\frac{1}{2 g_{Y M}^{2}} \int d^{4} x \operatorname{Tr}\left[\frac{-1}{2} F_{\mu \nu} F^{\mu \nu}+\mathcal{D}_{\mu} \Phi^{i} \mathcal{D}^{\mu} \Phi_{i}-\sum_{i<j}\left[\Phi_{i}, \Phi_{j}\right]^{2}\right]
$$

This theory theory is maximally supersymmetric, and has an $S U(2,2 \mid 4)$ superconformal symmetry group, with conformal subgroup $S U(2,2) \cong S O(2,4)$ and an R-symmetry subgroup $S U(4) \cong S O(6)$. Note that the $S U(2,2 \mid 4)$ symmetry group is exactly equal to the isometry group of the $\mathrm{AdS}_{5} \times \mathrm{S}^{5}$ supergravity background with the $F_{5}$ RR-flux. The $\mathrm{AdS}_{5}$ geometry captures the conformal symmetries of $\mathcal{N}=4 \mathrm{SYM}$, while the internal space $S^{5}$ captures its R-symmetry.

Furthermore, $\mathcal{N}=4 \mathrm{SYM}$ is conformal at the classical leverl, but is also believed to be conformal to all loop-orders, and therefore does not have any massive particle-like excitations. $\mathcal{N}=4$ SYM is therefore completely characterised by the rank of its $S U(N)$ gauge group $N$, and its Yang-Mills coupling $g_{Y M}$. We see from eq.(3.6) that this Yang-Mills coupling on the worldvolume action of the D3-branes is related to the string coupling by

$$
g_{Y M}^{2}=4 \pi g_{s}
$$

In the previous section we saw that the near-horizon limit of the $p$-brane geometry was obtained by taking for $\alpha \rightarrow 0$, keeping $\lambda=g_{s} N$ fixed. For $\mathcal{N}=4$ SYM this limit corresponds to the large $N$ limit, where we take $N \rightarrow \infty$, keeping the 't Hooft coupling $\lambda=g_{Y M}^{2} N$ fixed.

In this limit the theory simplifies considerably, as only the planar diagrams will survive. The non-planar diagrams will be suppressed with a power $N^{\chi}$, where $\chi$ is the genus of the diagram seen as a surface [68]. The large $N$ limit is in this sense reminiscent of a string theory, with a string coupling proportional to $N^{-1}$.

\subsubsection{The AdS/CFT Correspondence}

Maldacena made the relation between the large- $N$ limit of gauge theories and string theory more precise, by showing that the large- $N$ limit of $\mathcal{N}=4 \mathrm{SYM}$ indeed corresponds exactly to Type IIB superstring theory on $\mathrm{AdS}_{5} \times \mathrm{S}^{5}$. The two parameters of $\mathcal{N}=4 \mathrm{SYM}\left(g_{Y M}\right.$ and $\left.N\right)$ 

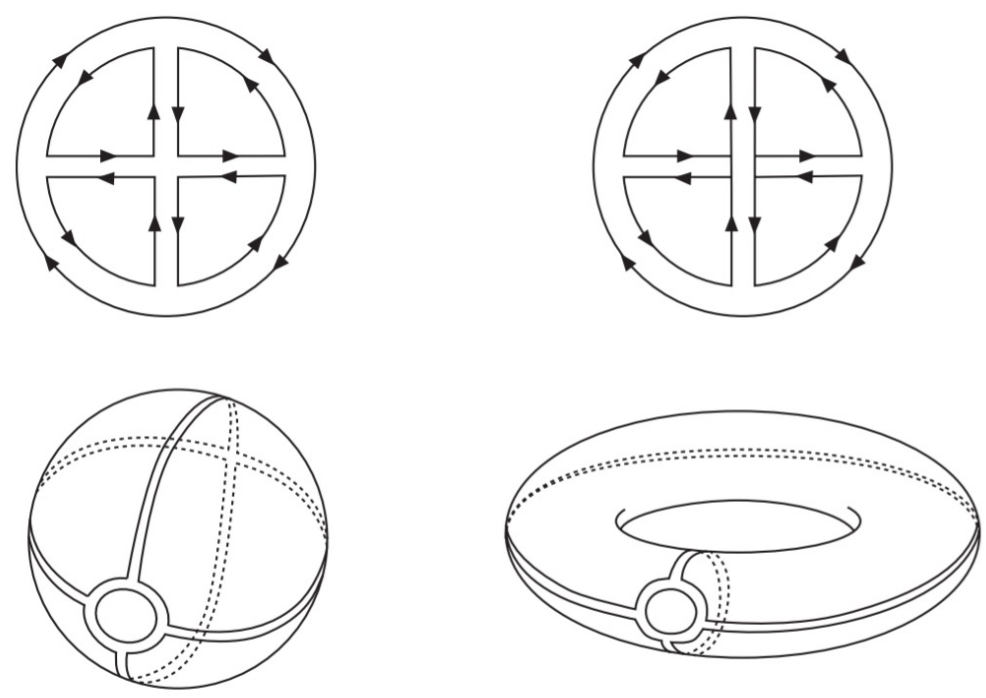

Figure 3.1: The diagram on the left is planar and has the topology of a sphere, while the diagram on the right is non-planar and has the topology of a torus. Image adapted from [149].

are now related to the parameters of the string theory $\left(\alpha^{\prime}\right.$ and $\left.g_{s}\right)$ as

$$
g_{Y M}^{2}=4 \pi g_{s}, \quad \lambda=L^{4} / \alpha^{\prime 2}
$$

where $\lambda$ is the 't Hooft coupling we introduced earlier, $g_{s}$ is the string coupling, and $L^{2} / \alpha^{\prime}=$ $L^{2} / \ell^{2}$ is the radius of the $\mathrm{AdS}_{5}$ and $\mathrm{S}^{5}$ geometries in units of string length.

The limit where we can solve the string theory is when $g_{s}$ is small, and the radius $L$ of the $\mathrm{AdS}_{5} \times \mathrm{S}^{5}$ spacetime is large compared to the string length $\alpha^{\prime} / L^{2} \rightarrow 0$. We now see that this solvable limit of strings on $\mathrm{AdS}_{5} \times \mathrm{S}^{5}$ corresponds exactly to the large $N$ limit of $\mathcal{N}=4 \mathrm{SYM}$ at large 't Hooft coupling $\lambda \rightarrow \infty$.

This is a very powerfull statement, as even though the large $N$ limit simplifies the gauge theory, it is still very difficult to perform calculations when the 't Hooft coupling deviates from zero. We now see that when the 't Hooft coupling goes all the way to infinity we can do these calculations in terms of supergravity excitations of the $\operatorname{AdS}_{5} \times \mathrm{S}^{5}$ geometry.

\subsection{Integrability and AdS/CFT}

In sections 2.1 and 2.1.1 we briefly introduced the concept of integrability for (two-dimensional) field theories. Here we will briefly explain how integrable structures show up in the large- $N$ limit of $\mathcal{N}=4 \mathrm{SYM}$. 


\section{ADS/CFT AND INTEGRABILITY FOR HALF-SUPERSYMMETRIC SCFTS}

In $\mathcal{N}=4 \mathrm{SYM}$ we can compose gauge invariant single trace operators by taking the trace of gauge covariant fields, all evaluated at the same point in spacetime

$$
\mathcal{O}(x)=\operatorname{Tr}\left[\Phi^{i}(x) \Phi^{j}(x) F_{\mu \nu}(x) \Phi_{\alpha}^{a}(x) \ldots\right] .
$$

When we consider the correlator of such a single trace operator with its adjoint $\langle\mathcal{O} \overline{\mathcal{O}}\rangle$, we find again that only the planar diagrams will contribute in the large $N$ limit. Note that though the theory simplifies tremendously in the large $N$ limit, we still have to include loop corrections to the different correlators once we turn on the 't Hooft coupling $\lambda \neq 0$.

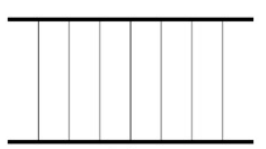

(a)

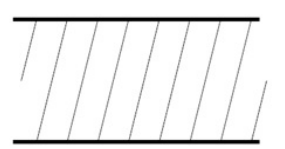

(b)

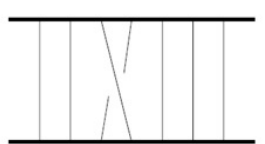

(c)

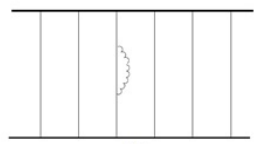

(d)

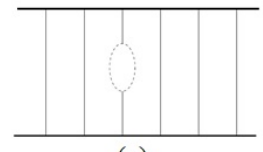

(e)

Figure 3.2: Different diagrams contributing to the correlators of single trace operators. The horizontal lines represent the single trace operators, and the vertical lines contractions of the different operators. The examples (a) and (b) are planar diagrams while (c) is not, and thus does not contribute in the large- $N$ limit. The diagrams (d) and (e) are examples of planar diagrams that contribute at one-loop order. Images adapted from [5].

In a CFT all of the two and three-point correlators we can construct from these single trace operators are further constrained by the conformal symmetries of the theory.

$$
\begin{aligned}
& \left\langle\mathcal{O}_{\Delta_{1}}\left(x_{1}\right) \mathcal{O}_{\Delta_{2}}\left(x_{2}\right)\right\rangle=\quad \frac{C_{12}}{x_{12}^{\Delta_{1}+\Delta_{2}}}, \\
& \left\langle\mathcal{O}_{\Delta_{1}}\left(x_{1}\right) \mathcal{O}_{\Delta_{2}}\left(x_{2}\right) \mathcal{O}_{\Delta_{3}}\left(x_{3}\right)\right\rangle=\frac{C_{123}}{x_{12}^{\Delta_{1}+\Delta_{2}-\Delta_{3} x_{23}^{\Delta_{2}}}{ }^{\Delta_{3}-\Delta_{1}} x_{13}^{\Delta_{1}+\Delta_{3}-\Delta_{2}}},
\end{aligned}
$$

where $x_{i j}=\left|x_{i}-x_{j}\right|$, and the $\Delta_{i}=\Delta_{i}^{(0)}+\gamma$ is the scaling dimension (the eigenvalue of the operator $\mathcal{O}_{\Delta}$ under the dilaton operator), with $\Delta^{(0)}$ the classical scaling dimension of the operator, and $\gamma$ the quantum corrections (also called the anomalous dimension). The $C_{i j \ldots n}$ are constants that depend on the gauge coupling $g_{Y M}$, rank of the gauge group $N$, and the scaling dimension of the different operators, and could be set to one by choosing a convenient normalisation for the $\mathcal{O}$. All higher order correlation functions of a CFT can be expressed in terms of these two- and three-point correlators using the operator product expansion.

For very small values of the 't Hooft coupling $\lambda \approx 0$ we simply have that $\Delta=\Delta_{0}$, but we would like to find exact expressions for the anomalous dimensions $\gamma$ of the different operators as a function of the 't Hooft coupling. It turns out that these anomalous dimensions of 
single trace operators can be obtained exactly, by identifying the single trace operators with spin chains [5]. At the $n$-th loop order this involves a spin chain where the sites interact with their $n$-th nearest neighbour. All of these spin chains turn out to be integrable, providing strong evidence for the integrability of the large- $N$ limit of $\mathcal{N}=4 \mathrm{SYM}$ to all orders in perturbation theory for all values of the 't Hooft coupling. See also $[9,6]$ and $[10]$ for extensive reviews.

The integrable structure in the large $N$ limit of $\mathcal{N}=4$ SYM in terms of spin chains (for arbitrary values of $\lambda$ ) translates on the string theory side to the integrable dynamics of free strings (for $g_{s}=0$ ) on $\mathrm{AdS}_{5} \times \mathrm{S}^{5}$ for arbitrary values of the AdS radius $L$. The integrability of the string worldsheet allows us to solve the dynamics of the string exactly. The energy spectrum of these free strings can be matched exactly to the spectrum of scaling dimensions of $\mathcal{N}=4$ SYM for all values of the 't Hooft coupling.

This has allowed for great progress in studying the lagre $N$ behaviour of various SCFTs, and their dual AdS geometries. See for example the case of $\mathrm{AdS}_{4} \times \mathrm{CP}_{3}$, dual to a $3 \mathrm{~d} \mathcal{N}=6$ supersymmetric Chern-Simons theory known as the ABJM model [11, 12].

In this thesis we are primarily interested in finding examples of new supergravity backgrounds on which strings are integrable, as this would imply the integrability of their dual SCFTs. Finding backgrounds on which the superstring is integrable becomes increasingly more difficult when these spaces (and their dual SCFTs) have fewer (super)symmetries. In the next sections we will introduce classes of half-supersymmetric AdS backgrounds and their dual SCFTs in various dimensions and their relation with integrability.

\subsection{Half-Supersymmetric Worldvolume Theories}

The example of AdS/CFT that we considered in the previous sections focussed on a maximally supersymmetric CFT in four dimensions. A lot of progress has been made over recent years in the study of half-supersymmetric D-dimensional quiver field theories, by studying the low energy fluctuations of Hanany-Witten brane set-ups [113] containing NS5-branes with stacks containing various numbers of $\mathrm{D}_{p}$-branes suspended between them. The literature on this topic is vast, and it is difficult to do it justice in this short introduction. We will present these constructions for various values of $p$ in more detail in section 3.6, with references [74]-[105]. The different stacks of $D_{p}$-branes result in an overall gauge group

$$
G=S U\left(N_{1}\right) \times S U\left(N_{2}\right) \times \ldots \times S U\left(N_{n-1}\right) \times S U\left(N_{n}\right) .
$$

Each of the $n D_{p}$-branes gives rise to a vector multiplet, transforming under the adjoint representation of the corresponding $S U\left(N_{i}\right)$ gauge group, describing the fluctuations of the $D_{p^{-}}$ brane stacks. In addition, there will be $n$ hyper multiplets, that transform as singlets under the $S U\left(N_{i}\right)$, and encode the fluctuations in the distance between the different NS5-branes. 


\section{ADS/CFT AND INTEGRABILITY FOR HALF-SUPERSYMMETRIC SCFTS}

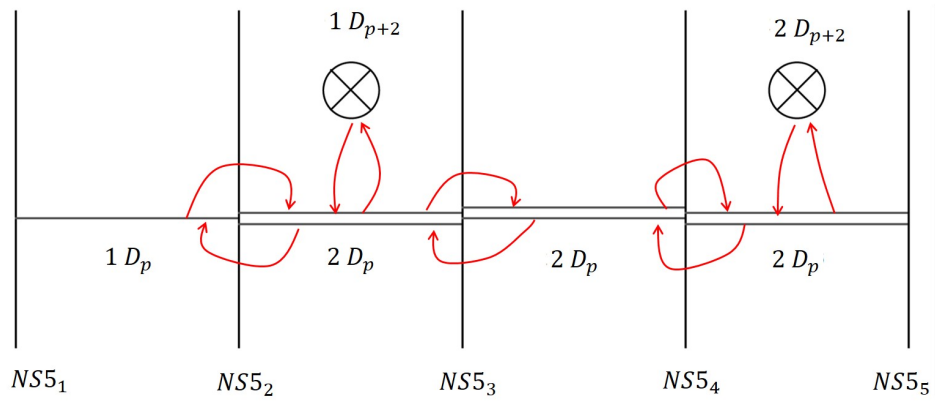

(a)

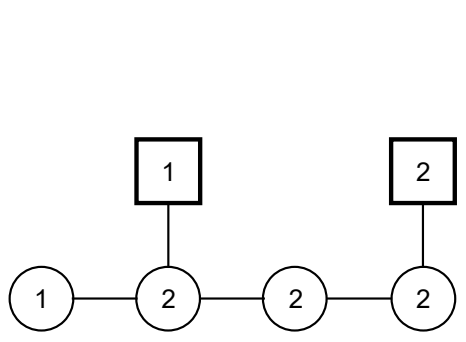

(b)

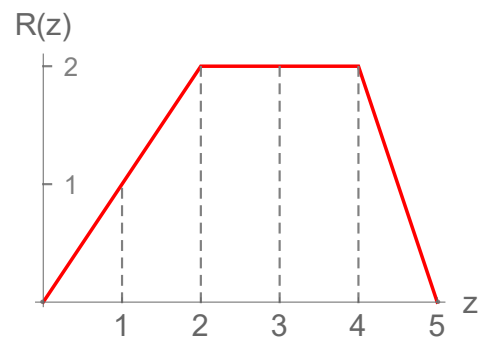

(c)

Figure 3.3: Example of a Hanany-Witten D6-D8-NS5 set-up, (a) brane set-up with strings going between the branes, cindicated by red arrows, giving rise to hypermultiplets. (b) The different number of gauge and flavour groups with their bifundamental fields can be summarised in a quiver diagram. (c) The corresponding rank function.

The quiver structure in these set-ups comes from strings going between the different Dbranes, and give rise to $n-1$ bifundamental hypermultiplets $\Psi$ that transform under the fundamental representation of one of the $S U\left(N_{i}\right)$ gauge groups, while their adjoint $\bar{\Psi}$ transforms under the anti-fundamental representation of a consecutive gauge group $S U\left(N_{i \pm 1}\right)$.

Flavours can be introduced by adding additional $D_{p+2}$-branes that do not extend between the NS5-branes, but extend on three additional directions perpendicular to the NS5-branes. As these $D_{p+2}$-branes are larger and heavier, their dynamics will freeze out. At low energies strings extending from one of the $D_{p}$-branes to these heavier $D_{p+2}$-branes will introduce additional flavour indices on the hypermultiplets. To keep track of all these different hypermultiplets one can summarise the colour and flavour groups in a quiver diagram, as is shown for an example in figure $3.3 \mathrm{~b}$. 


\subsubsection{Consistency Condition at the Conformal Fixed Point}

At the conformal fixed point the worldvolume theories are expected to be holographically dual to an $\mathrm{AdS}_{p+1}$ supergravity solution [3]. In the remainder of this section we will first focus in particular on the cases where $p=4,6 .^{2}$

For these $4 \mathrm{~d}$ and $6 \mathrm{~d}$ SCFTs the ranks of the different gauge and flavour groups $\left(N_{n}\right.$ and $F_{n}$ respectively) cannot be arbitrary at the conformal fixed point, ${ }^{3}$ but have to be related to each other such that

$$
\begin{aligned}
F_{n} & =2 N_{n}-N_{n+1}-N_{n-1} \\
& =\left(N_{n}-N_{n-1}\right)-\left(N_{n+1}-N_{n}\right) .
\end{aligned}
$$

This means the flavour groups act as the second derivative of the colour groups along the quiver. As a result, the colour groups and flavour groups of the quiver are fixed in terms of one another at the conformal fixed point.

It is expected that these $\mathrm{AdS}_{p+1}$ backgrounds arise as the near horizon limit of the brane setup when the distances between the different NS5-branes go to zero. The AdS geometry carries the information of the NS5 and $\mathrm{D}_{p}$-branes in the fluxes on the background. One can include the $D_{p+2}$ flavour branes in the AdS geometry by letting them backreact on the near-horizon 'colour' geometry [34]. The backreaction of these flavour branes will deform the supergravity geometry, which will now satisfy the equations of motion following from the action

$$
S_{\text {Type II }}+\int d z \delta^{9-(p+2)}\left(\vec{x}-\vec{x}_{D_{p+2}}\right) S_{B I W Z}
$$

where $S_{\text {Type II }}$ is the normal Type II supergravity action of eqs.(2.105) and (2.106), and $S_{B I W Z}$ is the Born-Infeld action for the $D_{p+2}$-flavour branes, amended with the appropriate WessZumino term, as we introduced in section 3.2. Since these flavour branes are localised, the Born-Infeld action has to be multiplied by a delta function. The BIWZ action is of the form

$$
S_{B I W Z}=-T_{p} \int d^{p+3} \sigma e^{-\Phi} \sqrt{-\operatorname{det}\left(g_{a b}-\mathcal{F}_{a b}\right)}+T_{p} \int \mathcal{C} \wedge e^{-\mathcal{F}}
$$

with $\mathcal{F}_{a b}, \Phi, g_{a b}$ and $B_{a b}$ the pullbacks of the supergravity fields induced by the background of the colour branes. The coupling to the RR-fields of the colour background is given by the WZ-part of the action.

\footnotetext{
${ }^{2}$ For $p=3,5$ the consistency condition of eq.(3.24) at the conformally fixed point is different. Because of this, care has to be taken for these cases as some of the results mentioned here and used throughout this thesis are no longer valid. We will comment on this in more detail in later sections.

${ }^{3}$ For $p=4$ the condition of eq.(3.24) will be equivalent to the vanishing of the beta-function. For $p=6$ this condition (3.24) ensures the vanishing of gauge anomalies in the $6 \mathrm{~d}$ SCFT.
} 


\section{ADS/CFT AND INTEGRABILITY FOR HALF-SUPERSYMMETRIC SCFTS}

The additional BIWZ term in the action (3.25) modifies the equations of motion. In particular, the Bianchi identities for the RR-fluxes that couple to the flavour brane will now be of the form

$$
d F_{8-(p+2)}=N_{f} \delta^{9-(p+2)}\left(\vec{x}-\vec{x}_{D_{p+2}}\right) .
$$

Since the $D_{p+2}$-flavour branes will act as sources for the $F_{8-(p+2)}$-flux, everywhere in the geometry the Bianchi identities $d F_{8-(p+2)}=0$ will be satisfied, except at the points where these flavour-branes are located.

If we demand that the consistency condition (3.24) is satisfied, we only need the ranks of the gauge groups to define a particular quiver (for $p=4,6$ ), the required flavour groups then follow from the consistency condition. Since the colour ranks contain all the information of the brane set-up, we expect the dual AdS geometry, including the backreaction of the flavour branes, to be completely determined by this rank function.

$$
R_{i}=\left(N_{1}, N_{2}, \ldots, N_{n-1}, N_{n}\right)
$$

This is indeed the case for the $\mathrm{AdS}_{7}$ backgrounds that we will introduce in more detail in section 3.6.1, and the $\mathrm{AdS}_{5}$ backgrounds in section 3.6.2. Given a particular rank function that should now be thought of as a piece-wise continuous linear function $R(z) \in C^{(0)}$ (see figure $3.3 \mathrm{c}$ ) - the dual geometries are completely determined.

We will see that in all these half-BPS backgrounds the coefficients in the metric, NS- and RR-fluxes are given in terms of the derivatives of one or multiple functions $\mathcal{F}_{i}\left(\Sigma_{2}\right)$, that have to satisfy a particular differential equation for the geometry to satisfy the BPS equations. The rank function of the quiver is then related to the boundary condition for this differential equation. We discuss these classes of half-BPS $\mathrm{AdS}_{p+1}$ backgrouds in more detail in section 3.6 .

\subsubsection{Supergravity Limit}

One way of obtaining a SCFTs for which the supergravity description is valid, is by considering very long quivers with large numbers for the colour and flavour groups. Here we will describe the conventional approach of obtaining long quivers, by 'scaling' an initial shorter quiver to one for which the supergravity description is valid.

\section{Scaling a Finite Quiver}

For the supergravity description to be a valid approximation of the dual SCFT the string coupling everywhere needs to be weak so we do not have to lift the ten-dimensional background to M-theory, furthermore the curvature needs to be small so we do not have to include stringy corrections of higher curvature terms in the effective supergravity action. 
In both the $\mathrm{AdS}_{5}$ backgrounds of eq.(3.35) and $\mathrm{AdS}_{7}$ backgrounds of eq.(4.6), we can scale the rank functions $R(z) \rightarrow N R(z)$ and obtain a different supergravity solution. This scaling cancels out in the metric, but multiplies the RR-fluxes with the same prefactor $N$, while the string coupling scales as $N^{-1}$. This scaling thus increases the number of $D_{p}$ colour and $D_{p+2^{-}}$ flavour branes, while simultaneously decreasing the string coupling $g_{s}^{2}$, keeping the number of NS5-branes fixed. By starting with a finite quiver this way and taking $N \rightarrow \infty$ we can take $g_{s}^{2} \rightarrow 0$, while keeping $g_{s}^{2} N$, as well as the overall shape of the quiver diagram fixed, see figure 3.4 .

$$
e^{2 \phi} \rightarrow \frac{1}{N} e^{2 \phi}, \quad F_{p} \rightarrow N F_{p}, \quad F_{p+2} \rightarrow N F_{p+2} .
$$

This limit decreases the closed string loop corrections (as we send $g_{s}^{2} \rightarrow 0$ ). Note that since the flavours and colours are related to one another by the consistency condition (3.24) this particular scaling multiplies the ranks of both the colour and flavour groups by $N$, while keeping their ratio fixed. ${ }^{4}$

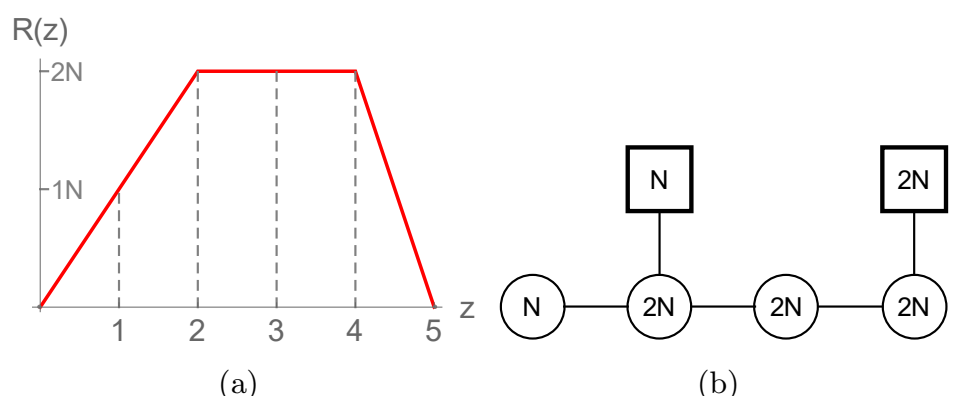

Figure 3.4: The quiver and rank function of figure 3.3, now rescaled with $R(z) \rightarrow N R(z)$.

We can alternatively scale the $z$-direction in the $\mathrm{AdS}_{5}$ and $\mathrm{AdS}_{7}$ solutions of eqs.(3.35) and (4.6). If we combine this with the $R(z) \rightarrow N R(z)$ scaling, we can increases the number of NS5and $\mathrm{D}_{p}$-colour branes in the corresponding set-up, while keeping the number of flavours-branes fixed, see figure 3.5. In addition to the string coupling getting suppressed, the internal direction on the space now grows, which reduces the curvature.

By combining these two scalings one can start from a finite quiver - like the one in figure 3.3 - and repeatedly scale it to large $N$ and large number of NS5-branes, to obtain a very long quiver for which the supergravity description is valid. The resulting final quiver has only a few localised flavour groups $F_{n}$, for which we are in the Veneziano limit where $F_{n} \rightarrow \infty$ and $N_{n} \rightarrow \infty$, with $F_{n} / N_{n}$ kept fixed.

\footnotetext{
${ }^{4}$ This in combination with $\lambda=g_{Y M}^{2} N \rightarrow \infty$ corresponds to taking the Veneziano limit, where the flavours $F_{n} \rightarrow \infty$ and $F_{n} / N_{n}$ is kept fixed (here $N_{n}$ and $F_{n}$ indicate the rank of the $n$-th colour and flavour group respectively).
} 


\section{ADS/CFT AND INTEGRABILITY FOR HALF-SUPERSYMMETRIC SCFTS}

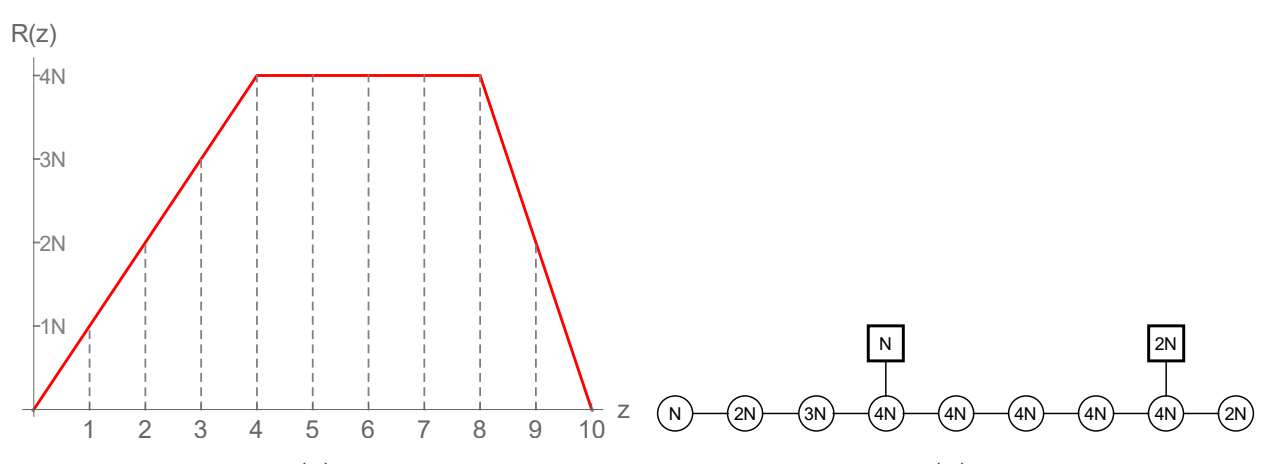

(a)

(b)

Figure 3.5: The quiver and rank function of figure 3.3, now with the combined scaling $R(z) \rightarrow$ $N R(z)$ and $z \rightarrow \omega z$. Note the flavour groups are the same as those in figure 3.4.

\subsection{Half-BPS AdS $_{p+1}$ Geometries}

We will list the different dimensional half-BPS $\mathrm{D}_{p}$ - $\mathrm{D}_{p+2}$-NS5-brane systems and their holographic duals that have been extensively studied in the literature. In all of the resulting $\mathrm{AdS}_{p+1}$ geometries, there is one direction $z$ on the internal space. All of the warp factors in these AdS geometries are completely determined by one or more functions $\mathcal{F}(z, \ldots)$ that always depends on $z$ (and possibly on an additional direction of the internal space), and geometrically encode the quiver structure of the corresponding brane set-up. In order to satisfy the BPS equations, these functions $\mathcal{F}$ have to satisfy a differential equation, where the rank function $R(z)$ is related to the boundary conditions of $\mathcal{F}$. For general $p$, the resulting geometries are of the form shown in table 3.1 .

\begin{tabular}{l|l}
$\mathbf{p}$ & $\mathbf{A d S}_{p+1}$ \\
\hline 6 & $\left(A d S_{7} \times S^{2}\right) \times_{w} \Sigma$ \\
\hline 5 & $\left(A d S_{6} \times S^{2}\right) \times_{w} \Sigma_{2}$ \\
\hline 4 & $\left(A d S_{5} \times S^{2} \times S^{1}\right) \times_{w} \Sigma_{2}$ \\
\hline 3 & $\left(A d S_{4} \times S^{2} \times S^{2}\right) \times_{w} \Sigma_{2}$
\end{tabular}

Table 3.1: Near horizon $A d S_{p+1}$ geometries for the different $\frac{1}{2}$ BPS $D_{p}-D_{p+2}$-NS5-brane systems.

The situation we have described so far in previous sections applies directly to the cases $p=6$ and $p=4$. For these $\mathrm{AdS}_{7}$ and $\mathrm{AdS}_{5}$ geometries, $R(z)$ is directly related to (the boundary values of) the derivatives of the functions $\mathcal{F}$. We show explicitly in both cases how one can define the supergravity solutions corresponding to continuous rank functions. 
The cases $p=3$ and $p=5$ are a bit different. The quiver structure of the dual field theories for these $\mathrm{AdS}_{4}$ and $\mathrm{AdS}_{6}$ geometries is encoded in the poles of the functions $\mathcal{F}$ at the boundary of $\Sigma_{2}$. This makes it not directly obvious how we could define the analogue of a continuous rank function for those cases. In addition, the consistency condition of eq.(3.24) that related the ranks of the flavour groups to the second derivative of colour rank function is no longer valid in these cases.

\subsubsection{Half-BPS AdS Geometries $_{7}$}

D6-D8-NS5 brane set-ups give rise to $6 \mathrm{~d} \mathcal{N}=(1,0)$ supersymmetric QFTs [113]. For conformal field theories the symmetry group of the worldvolume theory is enhanced to $O S p(2,6 \mid 2)$. These SCFTs are expected to have a Massive Type IIA dual description $[75,74,76]$ in terms of a geometry of the form

$$
\left(A d S_{7} \times S^{2}\right) \times_{w} \mathbb{R}_{z}
$$

where the metric and field content are given by

$$
\begin{aligned}
& d s^{2}=\sqrt{2} \pi\left(-\frac{\alpha}{\alpha^{\prime \prime}}\right)^{1 / 2}\left[8 d s_{A d S_{7}}^{2}+\frac{\alpha^{\prime \prime} \alpha}{\Delta} d \Omega_{2}^{2}+\frac{\alpha^{\prime \prime}}{\alpha} d z^{2}\right] \\
& B_{2}=\pi\left(\frac{\alpha \alpha^{\prime}}{\Delta}-z\right) d \Omega_{2}, \quad e^{\phi}=3^{4}\left(\left(2 \pi^{2}\right)^{5} \frac{\left(-\alpha / \alpha^{\prime \prime}\right)^{3}}{\Delta^{2}}\right)^{1 / 4}, \\
& F_{2}=\left(\frac{\alpha^{\prime \prime}}{162 \pi^{2}}+\frac{\pi F_{0} \alpha \alpha^{\prime}}{\Delta}\right) d \Omega_{2}, \quad F_{0}=F_{0}(z)
\end{aligned}
$$

Here $\Delta=\alpha^{\prime 2}-2 \alpha \alpha^{\prime \prime}$, and we defined $d \Omega_{2}^{2}=d \chi+\sin \chi^{2} d \xi$ and $d \Omega_{2}=\sin \chi d \chi \wedge d \xi$. The entire geometry is determined by the function $\alpha(z)$, which has to vanish at the end of the $z$-interval, and satisfy the differential equation

$$
\alpha^{\prime \prime \prime}(z)=-162 \pi^{3} F_{0}
$$

This function $\alpha(z)$ only depends on the $z$-direction, and is directly related to the rank function of the corresponding brane set-up as

$$
R(z)=\frac{-1}{81 \pi^{2}} \alpha^{\prime \prime}(z)
$$

When there is a finite number of flavour branes, $F_{0}$ is constant and discontinuous, and the corresponding function $\alpha(z) \in C^{(2)}$ is a piece-wise continuous third order polynomial in $z$. We will review these $\mathrm{AdS}_{7}$ solutions and their dual SCFTs in more detail in the next sections as well as the following chapters. 


\section{ADS/CFT AND INTEGRABILITY FOR HALF-SUPERSYMMETRIC SCFTS}

\subsubsection{Half-BPS $\mathrm{AdS}_{5}$ Geometries}

D4-D6-NS5 brane set-ups give rise to $4 \mathrm{~d} \mathcal{N}=2$ supersymmetric QFTs, like e.g. Seiberg-Witten theory [77] and Gaiotto theories [78], that can also be obtained from the reduction of a single M5-brane wrapping a punctured Riemann surface. At the conformal fixed point, the symmetry group is enhanced to $S U(2,2 \mid 2)$ and these set-ups are thought to have a near-horizon geometry [79] of the form

$$
\left(A d S_{5} \times S^{2} \times S^{1}\right) \times_{w} \Sigma_{2}
$$

with $\Sigma_{2}(z, \sigma)$ a two-dimensional Riemann surface. The NS-NS and RR-RR sectors are given by

$$
\begin{array}{rlrl}
d s^{2} & =\left(\frac{2 \dot{V}-\ddot{V}}{V^{\prime \prime}}\right)^{1 / 2}\left[4 d s_{A d S_{5}}^{2}+\frac{2 V^{\prime \prime} \dot{V}}{\Delta} d \Omega_{2}^{2}+\frac{2 V^{\prime \prime}}{\dot{V}}\left(d \sigma^{2}+d z^{2}\right)+\frac{4 V^{\prime \prime} \sigma^{2}}{2 \dot{V}-\ddot{V}} d \beta^{2}\right], \\
B_{2} & =2\left(\frac{\dot{V} \dot{V}^{\prime}}{\Delta}-z\right) d \Omega_{2}, & e^{\phi}=\left(4 \frac{(2 \dot{V}-\ddot{V})^{3}}{V^{\prime \prime} \dot{V}^{2} \Delta^{2}}\right)^{1 / 4} . \\
A_{1} & =2 \frac{2 \dot{V} \dot{V}^{\prime}}{2 \dot{V}-\ddot{V}} d \beta, & C_{3}=-4 \frac{\dot{V}^{2} V^{\prime \prime}}{\Delta} d \beta \wedge d \Omega_{2} .
\end{array}
$$

Note the entire geometry here is determined by the function $V(z, \sigma)$ and its derivatives. Here $\Delta=(2 \dot{V}-\ddot{V}) V^{\prime \prime}+\left(\dot{V}^{\prime}\right)^{2}$, and the dots and primes indicate the derivatives $\dot{V}=\sigma \partial_{\sigma} V$, and $V^{\prime}=\partial_{z} V$. The potential $V(z, \sigma)$ has to satisfy the Laplace equation

$$
\ddot{V}+\sigma^{2} V^{\prime \prime}=0
$$

supplemented by the boundary conditions

$$
\left.\sigma \partial_{\sigma} V\right|_{\sigma=0}=\lambda(z),\left.\quad V\right|_{\sigma \rightarrow \infty}=0 .
$$

These boundary conditions are now directly related to the rank function of the quiver structure in the corresponding brane set-up as

$$
\lambda(z)=R(z),
$$

and has to vanish at the beginning and end of the z-interval. See [80, 81, 135] for reviews. For a piece-wise continuous rank function, the solution for $V(z, \sigma)$ can be constructed as a superposition of Maldacena-Núñez solutions.

$$
\begin{aligned}
V_{M N}=\frac{1}{2} \sqrt{\sigma^{2}+(N+z)^{2}}-\frac{1}{2}(N+z) \sinh ^{-1}\left(\frac{N+z}{\sigma}\right) \\
-\frac{1}{2} \sqrt{\sigma^{2}+(N-z)^{2}}+\frac{1}{2}(N-z) \sinh ^{-1}\left(\frac{N-z}{\sigma}\right) .
\end{aligned}
$$


for which

$$
\dot{V}_{M N}=\frac{1}{2} \sqrt{\sigma^{2}+(N+z)^{2}}-\frac{1}{2} \sqrt{\sigma^{2}+(N-z)^{2}} .
$$

so that they correspond to the a rank function

$$
\lambda_{M N}(z)=\frac{1}{2}|N+z|-\frac{1}{2}|N-z|= \begin{cases}z & \text { if } \quad 0 \leq z \leq 1 \\ N & \text { if } \quad z \geq 1\end{cases}
$$

One can thus construct the function $V(z, \sigma)$ for a piece-wise linear rank function $R(z) \in C^{(2)}$ as a sum of these Maldacena-Núñez solutions.

\subsubsection{Notes on a class of Quarter-BPS $\mathrm{AdS}_{3}$ Geometries}

Let us briefly point out that the same logic also applies to the recently constructed class of quarter-BPS $\mathrm{AdS}_{3}$ geometries [104, 105]. These backgrounds are thought to be the near horizon limit of D2-D4-D6-D8-NS5 brane set-ups [103], which give rise to two-dimensional $\mathcal{N}=(0,4)$ QFTs. These theories have two sets of linear quivers, with colour groups $N_{n}, \tilde{N}_{n}$ and flavour groups $F_{n}, \tilde{F}_{n}$, coupled by matter fields. The colours and flavours of these two quivers cannot be independent of each other, but have to be chosen so that the gauge anomalies cancel. The condition for this is

$$
\tilde{F}_{n}=2 N_{n}-N_{n+1}-N_{n-1}, \quad F_{n}=2 \tilde{N}_{n}-\tilde{N}_{n+1}-\tilde{N}_{n-1} .
$$

Note this is analogous to the consistency condition in eq.(3.24), and our methods of defining infinite quivers in section 6.5.1 therefore apply to this case as well.

The supergravity solution is of the form

$$
\left(A d S_{3} \times S^{2} \times C Y_{2}\right) \times_{w} \mathbb{R}
$$

Here we will just give the expression for the metric. In addition this background contain a dilaton, $B_{2}$-field and RR $F_{0}, F_{2}$, and $F_{4}$-flux.

$$
d s^{2}=\frac{u}{\sqrt{\hat{h}_{4} h_{8}}}\left(d s_{A d S_{3}}^{2}+\frac{\hat{h}_{4} h_{8}}{4 \hat{h}_{4} h_{8}+\left(u^{\prime}\right)^{2}} d \Omega_{2}^{2}\right)+\sqrt{\frac{\hat{h}_{4}}{h_{8}}} d s_{C Y_{2}}^{2}+\frac{\sqrt{\hat{h}_{4} h_{8}}}{u} d z^{2}
$$

As in the other cases, the background is completely determined in terms of the functions $u(z)$, $\hat{h_{4}}(z)$ and $h_{8}(z)$, that have to be at most linear in $z$,

$$
u^{\prime \prime}=0, \quad h_{8}^{\prime \prime}=0, \quad \hat{h}_{4}^{\prime \prime}=0 .
$$

The functions $\hat{h}_{4}(z)$ and $h_{8}(z)$ are directly related to the rank functions of these two quivers as

$$
\hat{h}_{4}(z)=R(z), \quad h_{8}(z)=\tilde{R}(z)
$$




\section{ADS/CFT AND INTEGRABILITY FOR HALF-SUPERSYMMETRIC SCFTS}

\subsubsection{Half-BPS $\mathrm{AdS}_{4}$ Geometries}

For the $\mathrm{AdS}_{p+1}$ duals of the $\mathrm{D}_{p}-\mathrm{D}_{p+2}$-NS5 brane set-ups the situation for $p=3$ and $p=5$ is different from $p=4$ and $p=6$ that we have discussed in this chapter so far. The quiver structure of the dual field theories is for these $\mathrm{AdS}_{4}$ and $\mathrm{AdS}_{6}$ geometries encoded in the poles of the functions $\mathcal{F}$ at the boundary of $\Sigma_{2}$. In addition, the consistency condition of eq.(3.24) that related the ranks of the flavour groups to the second derivative of colour rank function is no longer valid in these cases.

D3-D5-NS5 brane set-ups give rise to $3 \mathrm{~d} \mathcal{N}=4$ supersymmetric, mirror symmetric gauge theories known as $T_{\hat{\rho}}^{\rho}(S U(N))$-theories, introduced in [85]. These theories are characterised by two partitions $\rho$ and $\hat{\rho}$ of an integer $N$, that one can relate to a corresponding linear quiver diagram as,

$$
\begin{aligned}
& \rho=\{\underbrace{\ell^{(1)}, \ldots \ell^{(1)}}_{N_{D 5}^{(1)}}, \ldots, \underbrace{\ell^{(n)}, \ldots \ell^{(n)}}_{N_{D 5}^{(n)}}, \ldots, \underbrace{\ell^{(p)}, \ldots \ell^{(p)}}_{N_{D 5}^{(p)}}\} \\
& \hat{\rho}=\{\underbrace{\ell^{(1)}, \ldots \hat{\ell}^{(1)}}_{N_{N S 5}^{(1)}}, \ldots, \underbrace{\hat{\ell}^{(n)}, \ldots \hat{\ell}^{(n)}}_{N_{N S 5}^{(n)}}, \ldots, \underbrace{\hat{\ell}^{(p)}, \ldots \hat{\ell}^{(p)}}_{N_{N S 5}^{(p)}}\} .
\end{aligned}
$$

These theories have an IR conformal fixed point when

$$
F_{n}>2 N_{n}-N_{n+1}-N_{n-1} .
$$

This now implies the values of the flavour groups have to be larger than the second derivative of the rank function.

At the conformal fixed point the symmetry group is enhanced to $O S p(2,2 \mid 4)$, and the system is thought to have a Type IIB dual description in terms of

$$
\left(A d S_{4} \times S^{2} \times S^{2}\right) \times_{w} \Sigma_{2}
$$

where $\Sigma_{2}$ has the topology of a disk. When we parametrise $\Sigma_{2}$ in terms of a complex coordinate $z$, the geometry is given by $[86,87]$

$$
d s^{2}=2\left(\frac{N_{1} N_{2}}{W^{2}}\right)^{1 / 4}\left[d s_{A d S_{4}}^{2}+\frac{h_{1}^{2} W}{N_{1}} d s_{\Omega_{1}^{2}}^{2}+\frac{h_{2}^{2} W}{N_{2}} d s_{\Omega_{2}^{2}}^{2}+\frac{2 W}{h_{1} h_{1}} d z d \bar{z}\right]
$$

Here we omitted the expressions for the dilaton, $B_{2}$-field, and $F_{3}$ and $F_{5}$ flux as we will not perform any calculations on this geometry. See also $[88,89]$ for extensive reviews.

The geometry is completely specified in terms of two harmonic functions $h_{1}(z, \bar{z})$ and $h_{2}(z, \bar{z})$, and their derivatives, with $W=\partial \bar{\partial}\left(h_{1} h_{2}\right), N_{1}=2 h_{1} h_{2}\left|\partial h_{1}\right|^{2}-h_{1}^{2} W$ and $N_{2}=$ 
$2 h_{1} h_{2}\left|\partial h_{2}\right|^{2}-h_{2}^{2} W$. These functions only depend on $\Sigma_{2}$, that in these coordinates corresponds to a strip of the upper-half complex plane

$$
\Sigma=\left\{z \in \mathbb{C} \mid 0 \leq \operatorname{Im} z \leq \frac{\pi}{2}\right\}
$$

with the two sphere vanishing at $\operatorname{Im} z=0, \frac{\pi}{2}$. The solutions for $h_{1}$ and $h_{2}$ can be written as a sum over harmonic functions of the form

$$
\begin{aligned}
& h_{1}=\left[-i \alpha_{1} \sinh \left(z-\beta_{1}\right)-\gamma_{1} \ln \tanh \left(\frac{i \pi}{4}-\frac{z-\delta_{1}}{2}\right)\right]+c . c . \\
& h_{2}=\left[\alpha_{2} \cosh \left(z-\beta_{2}\right)-\gamma_{2} \ln \tanh \left(\frac{z-\delta_{2}}{2}\right)\right]+c . c .
\end{aligned}
$$

with the $\left(\alpha_{i}, \beta_{i}, \gamma_{i}, \delta_{i}\right)$ real parameters. The quiver structure of the field theory is now encoded in the poles on the upper and lower boundary of $\Sigma_{2}$ of these functions, with the residues of the poles of $h_{1}$ and $h_{2}$ giving respectively the NS5 and D5 fluxes.

\subsubsection{Half-BPS AdS $_{6}$ Geometries}

The discussion we will present here for the $\mathrm{AdS}_{6}$ geometries follows the lines of the previous section on $\mathrm{AdS}_{4}$ geometries. The D5-D7-NS5 brane set-ups are a bit more complicated. Charge conservation does not allow D5-brane's to end on an NS5-brane, and at the intersection point, the branes will instead attract each other and merge to form a $(\mathrm{p}, \mathrm{q}) 5$-brane, which allows the formation of so-called (p,q)-5-brane webs [95, 94].

We can still consider a particular brane-web that arises from a linear quiver diagram, but the condition for these theories to have a conformal fixed point will now be $[96,97]$

$$
F_{n} \leq 2 N_{n}+4
$$

This implies the values of the flavour groups are no longer related to the second derivative of the rank function.

These set-ups give rise to $5 \mathrm{~d} \mathcal{N}=1$ supersymmetric QFTs. At the conformal fixed point the symmetry group is enhanced to $F(4)$ (the unique superconformal group in 5 dimensions). It is thought these SCFTs have a dual description in terms of a Type IIB geometry of the form

$$
\left(A d S_{6} \times S^{2}\right) \times_{w} \Sigma_{2}
$$

Where $\Sigma_{2}$ has the topology of a disk. We can again choose coordinates where $\Sigma_{2}$ is parameterised by a complex coordinate $z$. These Type IIB backgrounds with an $F(4)$ isometry group were given in $[98,99,100]$.

$$
d s^{2}=f_{6}^{2} d s_{A d S_{6}}^{2}+f_{2}^{2} d \Omega_{2}^{2}+d s_{\Sigma}^{2}
$$




\section{ADS/CFT AND INTEGRABILITY FOR HALF-SUPERSYMMETRIC SCFTS}

Here we largely omit the details of these backgrounds as their expressions are rather complex. See [102] for a short review. These $\mathrm{AdS}_{6}$ backgrounds have an NS-NS sector with dilaton and $B_{2}$-flux, the RR-RR sector consists of an $F_{3}$ and $F_{5}$-flux.

As before, all functions of the geometry are completely determined in terms of two locally holomorphic functions $\mathcal{A}_{ \pm}$that only depend on the complex coordinate $z$. Analogous to the earlier discussed $\mathrm{AdS}_{4}$ backgrounds, the quiver structure will be encoded in terms of the poles of these functions $\mathcal{A}_{ \pm}$on $\Sigma_{2}$. The charges of the $(p, q)$ 5-branes will correspond to the residues of these poles, while the D7-branes are related to punctures of $\Sigma_{2}$.

\subsection{Integrability in $\frac{1}{2}$ BPS Supergravity Solutions}

Of these different classes of half-BPS supergravity solutions the first that was realised was the Gaiotto-Maldacena classification of $\mathrm{AdS}_{5}$ solution [79]. In [190] it was shown that the nonAbelian T-dual of the $\mathrm{AdS}_{5} \times \mathrm{S}^{5}$ solution (where a NATD is performed on one of the $S U(2)$ isometries inside the $S O(6)$ isometry group) is a specific solution in this class of $\mathrm{AdS}_{5}$ geometries, known as the Sfetsos-Thompson solution. We briefly consider this Sfetsos-Thompson background in more detail in section 7.0.2.

The superstring on $\mathrm{AdS}_{5} \times \mathrm{S}^{5} \cong S U(2,2 \mid 4) /(S O(1,4) \times S O(5))$ is well known to be integrable $[6,9]$ (as is the dual $\mathcal{N}=4$ SYM theory in the large- $N$ limit [7]). Since we have seen in section 2.8.3 that (non-)Abelian T-duality preserves the integrable structure of the string worldsheet, we know the dynamics of the superstring on the Sfetsos-Thompson background (as well as its dual $4 \mathrm{~d} \mathcal{N}=2$ SCFT in the large- $N$ limit) has to be integrable as well.

The field theory that is dual to the Sfetsos-Thompson background was further studied in [197], where it was shown how the Sfetsos-Thompson background has a potential $V(z, \sigma)$ (see eq.(3.35)) that corresponds to an infinitely long linear quiver

$$
V_{N A T D}(z, \sigma)=z\left(\log \sigma-\frac{\sigma^{2}}{2}\right)+\frac{z^{3}}{3}, \quad \lambda(z)=z .
$$

This means the ranks of the gauge groups increase linearly, and are of the form $U(1) \times S U(2) \times$ $S U(3) \times S U(4) \times S U(5) \times \ldots$. Since this quiver does not end, the central charge of the dual field theory would be infinite, and hence it is not clear whether the Sfetsos-Thompson background is dual to a well defined quantum field theory. In [197] this is examined further by 'completing' the quiver with the addition of a flavour group, so that its length becomes finite and quiver terminates with the flavour group.

Similarly the Abelian T-dual of $\mathrm{AdS}_{5} \times \mathrm{S}^{5}$ corresponds to a flat quiver where the end points have to be identified (making it circular).

$$
V_{A T D}(z, \sigma)=\log \sigma-\frac{\sigma^{2}}{2}+z^{2}, \quad \lambda(z)=1
$$




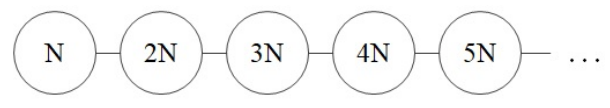

(a)

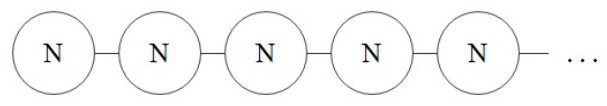

(b)

Figure 3.6: The quivers of the $\mathrm{AdS}_{5}$ Gaiotto-Maldacena geometries that correspond to the non-Abelian T-dual (left, infnitely long linear quiver) and (Abelian) T-dual (flat quiver where the beginning and end can be identified), of $\mathrm{AdS}_{5} \times \mathrm{S}^{5}$.

Similar to the non-Abelian T-dual we then find that the warp factor in eq.(3.35) vanishes for this background, as the potential satisfies $4 \dot{V}-\ddot{V}=V^{\prime \prime}$. The dynamics of the string on the $\mathrm{AdS}_{5}$ space thus decouples from its motion on the internal space and it can be shown that the dynamics of classical strings on this background looks integrable. This is to be expected as this background is the Abelian T-dual of $\mathrm{AdS}_{5} \times \mathrm{S}^{5}$. Unlike the infinite linear quiver, the corresponding SCFT now does have a finite central charge [197]. A more extensive study into the integrability of all the other $\mathrm{AdS}_{5}$ backgrounds in the Gaiotto-Maldacena classification was later undertaken in [140], and [147], where the authors concluded from various analytical and numerical methods that indeed the Sfetsos-Thompson background and the circular quiver are is integrable, whereas all other backgrounds that contain flavour groups show signs of nonintegrable dynamics for the string worldsheet.

We will conclude this chapter by summarising the known results for integrability in the other classes of half-BPS supergravity backgrounds with an $\mathrm{AdS}_{p+1}$ factor. Our results are summarised in figure 3.7 .

Similarly in the $\mathrm{AdS}_{4}$ case, the infinite linear quiver background can be obtained from a non-Abelian T-duality on a Type IIA background that is a reduction of the $\mathrm{AdS}_{4} \times \mathrm{S}^{7}$ M-theory background [198]. By writing $\mathrm{S}^{7}=\mathbb{R}_{\theta} \times{ }_{w} \mathrm{~S}_{1}^{3} \times{ }_{w} \mathrm{~S}_{2}^{3}$ as two three-spheres fibred over a angle $\theta$, and reducing along the Hopf direction on one of these three-spheres $\mathrm{S}_{2}^{3}$ we obtain

$$
d s_{10}^{2}=\cos \frac{\theta}{2}\left[d s_{A d S_{4}}^{2}+\left(d \theta^{2}+4 \sin ^{2} \frac{\theta}{2} d s_{S_{1}^{3}}^{2}+\cos ^{2} \frac{\theta}{2} d s_{S^{2}}^{2}\right)\right] .
$$

This particular reduction breaks 16 of the 32 supersymmetries of the original $\mathrm{AdS}_{4} \times \mathrm{S}^{7}$ background and leaves us with an $O S p(2,2 \mid 4)$ isometry group. ${ }^{5}$ To our knowledge it is not known whether the string worldsheet is still integrable on the remaining background, though the warp factors seem to suggest it might not be possible to use a coset construction to obtain this target space it would be very interesting to study this in further detail.

By performing a NATD on the $\mathrm{S}_{1}^{3}$ we do not break any further supersymmetries, as the

\footnotetext{
${ }^{5}$ Note this is a different reduction of $\mathrm{AdS}_{7} \times \mathrm{S}^{4}$ than the $\mathrm{AdS}_{4} \times \mathrm{CP}^{3} \cong O S p(2,2 \mid 6) /(S O(1,3) \times U(3))$ background that preserves 24 supersymmetries and is known to be integrable [cite]
} 


\section{ADS/CFT AND INTEGRABILITY FOR HALF-SUPERSYMMETRIC SCFTS}

Killing spinors of this background are independent of $S_{1}^{3}$. The resulting background fits in the ABEG classification of half-BPS $\mathrm{AdS}_{4}$ backgrounds, and is dual to an infinitely long linear quiver (not identical but) in a sense similar to the Sfetsos-Thompson background [198].

Similarly, the Abelian T-dual along the Hopf direction on the $S^{3}$ results in a solution with a circular quiver, which has been extensively studied in [191]. It would be very interesting to study the integrability of the string worldsheet on these backgrounds in further detail.

Similarly, one can start from the the warped $\mathrm{AdS}_{6} \times{ }_{w} \mathrm{~S}^{4}$ Brandhuber-Oz solution [193] that arises as the near-horizon solution of D4-branes at an O8-plane with D8-flavour branes.

$$
d s_{10}^{2}=\frac{1}{4} \cos ^{-\frac{1}{3}} \theta\left(9 d s_{A d S_{6}}^{2}+4 d s_{S^{4}}^{2}\right),
$$

where $d s_{S^{4}}^{2}=d \theta^{2}+\sin ^{2} \theta d s_{S^{3}}^{2}$, and $\theta \in\left[0, \frac{\pi}{2}\right]$, with an additional dilaton $\Phi$, Romans mass $F_{0}$ and $F_{4}$-flux. This background has an $F(4)$ isometry group, but again the warpfactor makes it doubtful whether the entire background could be realised as a coset construction and if the string worldsheet could be integrable on this background.

By performing an Abelian T-duality along the Hopf direction of the $\mathrm{S}^{3}$ a solution is obtained that arises as the near horizon geometry of smeared D5, NS5's and D7/O7 branes [194, 195]. This background has an $F(4)$ isometry group and fits in the DGU classification of half-BPS $\mathrm{AdS}_{6}$ solutions. Since we would expect the original background to be non-integrable, we would then expect this background to also be non-integrable.

By performing a NATD on an $S U(2)$ subgroup of the $S O(4)$ isometry group of this background, ${ }^{6}$ another supergravity background is obtained that is suggestive of a linear quiver with gauge groups of increasing rank [194, 195]. Again we would not expect strings on the resulting target space to be integrable as we would also not expect them to be on the original geometry before we performed the non-Abelian T-duality.

We have summarise these results in figure 3.7. Because the half-BPS classes of $\mathrm{AdS}_{4}$ and $\mathrm{AdS}_{6}$ geoemtries are written in more complicated terms than the $\mathrm{AdS}_{5}$ geometries (where the entire background is determined by a single function $V(\bar{z}, \sigma))$ there has not yet been a study for the (non)-integrable dynamics of classical strings on all of the AdS solutions in these classifications. It would still be very interesting to do so. Perhaps, as one learns more about these classes of half-BPS supergravity backgrouns, it might be possible perform such a study on the string worldsheet in a more convenient set of variables for these backgrounds. In chapter 5 we will perform such a detailed study for the dynamics of the string worldsheet on all of the half-BPS geometries with an $\mathrm{AdS}_{7}$ factor in the Tomasiello-Cremonesi classification.

\footnotetext{
${ }^{6}$ there is no $S O(5)$ isometry group as the $\mathrm{AdS}_{6}$ space is warped along one of the angles on the $\mathrm{S}^{4}$.
} 


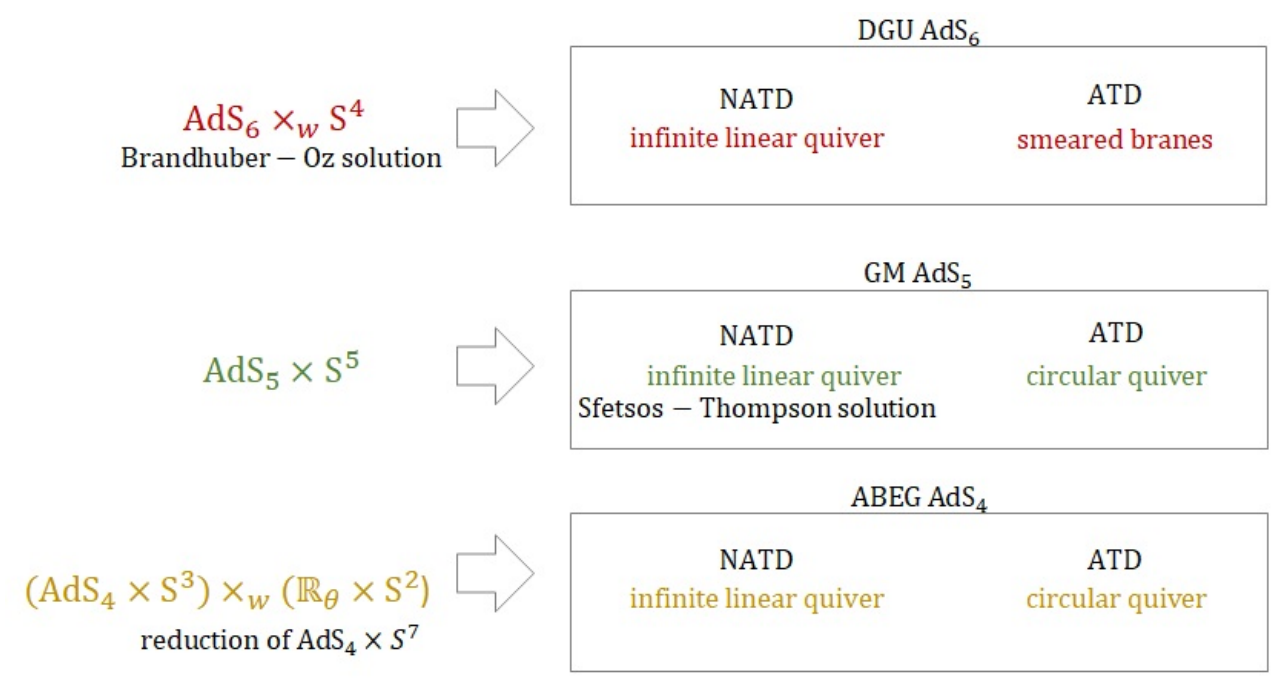

Figure 3.7: The different classes of half-BPS $\mathrm{AdS}_{p+1}$ solutions with corresponding quiver structure that can be obtained by performing a (non)-Abelian T-duality on other known solutions. The solutions are written in different colours depending on whether they are known to be integrable (green), thought to be non-integrable (yellow), and strongly suspected to be nonintegrable (red). 
IV. $\mathrm{ADS}_{7}$ DUALS OF $6 \mathrm{D} \mathcal{N}=(1,0)$ SCFTS

\section{Chapter 4}

\section{$\mathbf{A d S}_{7}$ duals of $\mathbf{6 d} \mathcal{N}=(1,0)$ SCFTs}

In this chapter we will give a detailed introduction to the $6 \mathrm{~d} \mathcal{N}=(1,0)$ SCFT's and their holographic $\mathrm{AdS}_{7}$ description $[75,74,76]$ that we briefly introduced earlier in section 3.6.1. We will give a detailed overview of the field content for these theories, and their relation to the D6-D8-NS5 systems briefly discussed earlier in section 3.6.

Our contributions in this chapter are of a reviewing nature and based on the first part of [136], where we showed how one can easily read off the quiver structure of the dual field theory from the internal space of the geometry, using the coordinates introduced in [160]. We illustrate this here with a wide range of examples.

We will use these $\mathrm{AdS}_{7}$ backgrounds in the following chapter to study the (non)-integrability of strings on these backgrounds, allowing us to make statements on the (non)-integrability of the dual $6 \mathrm{~d} \mathcal{N}=(1,0)$ SCFTs.

\section{$4.16 \mathrm{~d} \mathcal{N}=(1,0)$ SCFTs}

Let us first point out that in dimension higher than four, most terms we can write in action become strongly coupled and non-renormalisable at higher energies (similar to QED in 4 dimensions). The Wilsonian logic, according to which we start from a conformal (not necessarily weakly coupled) field theory in the UV and deform it by inserting relevant operators into the Lagrangian, flowing to interesting field theories at low energies, does not apply here.

As an example, let us first consider a simple interacting field theory in six dimensions with action,

$$
S=\int d^{6} x\left[-\frac{1}{2}\left(\partial_{\mu} \phi\right)^{2}-V(\phi)\right]
$$

Here $\phi$ is a real scalar field with mass dimension $[\phi]=m^{2}$. The potential can be a mass term $V=\frac{m^{2}}{2} \phi^{2}$ (or more interestingly a classically marginal interaction term, like $V=g \phi^{3}$, but this would lead to a system without ground state). A potential like $V=\lambda \phi^{4}$ has a well-defined 
vacuum, but the interaction is irrelevant, hence the theory is not well defined without a UV completion. The same reasoning applies to all of the higher interaction terms we could write down, suggesting that the only renormalisable theory for a scalar field in six dimensions can be at most a non-interacting massive scalar field.

Nevertheless, different string theoretic constructions have suggested that supersymmetric field theories of scalars coupled to gauge fields have an interacting UV fixed point. In terms of a Lagrangian description such theories would be of the form

$$
\mathcal{L} \sim-\frac{1}{2}\left(\partial_{\mu} \phi\right)^{2}-c \phi F_{\mu \nu}^{2}+\text { fermions. }
$$

Since the scalar $\phi$ takes the role of the inverse coupling of the gauge theory, we are dealing with the strong coupling limit of a gauge field theory when $\langle\phi\rangle \rightarrow 0$. The presence of fermions in the supersymmetric theory implies the possible existence of gauge anomalies that need to be cancelled. This cancellation is possible if the scalar $\phi$ belongs to a tensor multiplet [154], [164] (see below for a description of the relevant multiplets) and a certain tuning between the amount of adjoint and fundamental matter must be imposed.

This picture was realised in brane constructions. The relevant Hanany-Witten set-ups [155] were presented in [113]. The associated field theories preserve eight Poincare supercharges, have $S O(1,5)$ Lorentz and $S U(2)$ R-symmetries. In more detail, the field theories with $\mathcal{N}=(1,0)$ SUSY are constructed in terms of the following multiplets:

- Tensor multiplets with field content $\left(B_{\mu \nu}, \lambda_{1}, \lambda_{2}, \phi\right)$. A two form with self-dual curvature $H_{3}=d B_{2}$, two fermions and a real scalar.

- Vector multiplets with field content $\left(A_{\mu}, \hat{\lambda}_{1}, \hat{\lambda}_{2}\right)$, a six-dimensional vector and two fermions.

- Hypermultiplets with field content $\left(\varphi_{1}, \varphi_{2}, \psi_{1}, \psi_{2}\right)$, two scalars and two fermions.

- Linear multiplets with field content $(\vec{\pi}, c, \tilde{\xi})$ an $S U(2)$ triplet and a singlet, together with a fermion.

To reproduce the Lorentz and R-symmetry mentioned above, the authors of [113] distributed D6, NS5, and D8 branes according to Table 4.1. The resulting field theories have a 'tensor branch' when the scalar $\phi$ gets a non-zero VEV. In this case, the $S U(2)_{R}$ symmetry is preserved. When the scalars inside the hyper or the linear multiplet get VEVs, we are instead in the Higgs branch, where the R-symmetry is broken. In what follows we will be concerned with the tensor branch only:

When the NS5-branes are separated, the system is in the tensor branch (where the difference between the scalars in the different tensor multiplets $\left\langle\phi_{i}-\phi_{i-1}\right\rangle$ is non-zero). The bosonic part 
of the action for the resulting 6d QFTs will be of the form [131]

$$
\begin{aligned}
S_{\text {vec }+ \text { ten }}^{\text {bos }}=\int\left[\frac{1}{2} d \phi \wedge \star d \phi+\frac{1}{2} H \wedge \star H\right] & \\
& +\sqrt{c} \int[-\phi \operatorname{Tr}(F \wedge \star F)+B \wedge \operatorname{Tr}(F \wedge F)]+S_{S C}
\end{aligned}
$$

where $S_{C S}$ is a Chern-Simons term, and

$$
H=\mathrm{d} B+\sqrt{c} \operatorname{Tr}\left(A \wedge \mathrm{d} A-\frac{2}{3} A \wedge A \wedge A\right)
$$

The group under which the 3-form field strength $H$ transforms will (when the NS5-branes are seperated) be broken to an Abelian gauge group $\mathrm{U}(1)^{n}$ when we have $n$ NS5-branes. We have to impose the self duality of the $H_{3}$ field strength by hand. The equation of motion for the $H_{3}$ field will then be of the form

$$
d H=\mathrm{d} \star H=\sqrt{c} \operatorname{Tr}(F \wedge F)
$$

which implies the field configurations with non-zero $\operatorname{Tr}(F \wedge F)$ - the instanton strings - will be the sources for the $H_{3}$ field.

\begin{tabular}{c||cccccc|c|ccc} 
& $t$ & $x_{1}$ & $x_{2}$ & $x_{3}$ & $x_{4}$ & $x_{5}$ & $x_{6}$ & $x_{7}$ & $x_{8}$ & $x_{9}$ \\
\hline \hline NS5 & $\bullet$ & $\bullet$ & $\bullet$ & $\bullet$ & $\bullet$ & $\bullet$ & $\cdot$ & $\cdot$ & $\cdot$ & $\cdot$ \\
\hline \hline D6 & $\bullet$ & $\bullet$ & $\bullet$ & $\bullet$ & $\bullet$ & $\bullet$ & $\bullet$ & $\bullet$ & $\cdot$ & $\cdot$ \\
\hline D8 & $\bullet$ & $\bullet$ & $\bullet$ & $\bullet$ & $\bullet$ & $\bullet$ & $\cdot$ & $\bullet$ & $\bullet$ & $\bullet$
\end{tabular}

Table 4.1: The generic brane set-ups. All the branes are extended on the Minkowski $R^{1,5}$ directions. The D6-branes also extend over $x_{6}$ where they have finite size extension between NS5-branes. The D8-branes also extend along the $x_{7}, x_{8}$ and $x_{9}$ directions, preserving the $S O(3)_{R}$ symmetry. 
There are some key differences with the $\mathrm{D}_{p}-\mathrm{D}_{p+1}$-NS5 Hanany-Witten set-ups in lower dimensions.

- The dimension of the field theory on the NS5-branes is the same as that on the D6branes that are confined between the NS5-branes. The non-decoupling of the five-branes dynamics adds the dynamical tensor multiplets to the field theories. These are absent in lower dimensional set-ups.

- The bending of the NS5-branes due to other $p$-branes ending on them leads to a Laplace equation in $6-p$ dimensions. In this case, where $p=6$, there is no-bending. The field content is always such that anomalies are cancelled, namely

$$
N_{D 6, R}+N_{D 6, L}+N_{D 8}=2 N_{D 6, c},
$$

being $N_{D 6, R / L}$ the number of sixbranes to the right/left of a given stack with $N_{D 6, c}$ branes.

- The worldvolume theory of the NS5-branes contains charged defects that can be identified with the instantonic strings charged under the self-dual $H_{3}$ in eq.(4.4).

- When the system is in the tensor branch the instantonic strings are massive and the field theory can be described by an anomaly-free quiver.

\subsubsection{Non-Lagrangian Conformal Fixed Point}

The action in eq.(4.3) is for the tensor branch - when the NS5-branes are separated - and does not describe a conformal field theory. The theory is proposed [154] to flow to a strongly coupled six dimensional SCFT with $(1,0)$ SUSY when we go to the origin of the tensor branch $\left\langle\phi_{i}-\phi_{i-1}\right\rangle \rightarrow 0$. These are the theories that we will study the remainder of this thesis. There are several peculiarities that make it not possible to write a conventional Lagrangian description for these theories:

- Note that in the second term of eq.(4.3) $\phi$ shows up as the inverse Yang-Mills coupling constant for the vector field $A_{\mu}$ in the vector multiplet. When $\left\langle\phi_{i}\right\rangle \rightarrow 0$ the YangMills field becomes infinitely strongly coupled, and the corresponding terms in the action become singular. This makes it seemingly impossible to treat the conformal fixed point perturbatively.

- As the distances between the NS5-branes, corresponding to $\left\langle\phi_{i}-\phi_{i-1}\right\rangle$, go to zero, the gauge group of the tensor field $B_{\mu \nu}$ is enhanced from $U(1)^{n}$ to $S U(N)$. It is at present unclear how to write a field strength for a 2-form gauge field with a non-Abelian gauge group. This problem can be seen intuitively by considering, analoguous to the nonAbelian field strength for a 1-form gauge field, an ansatz like $H_{\mu \nu \rho}=\partial_{[\mu} B_{\nu \rho]}+\left[B_{\mu \nu}, B_{\rho \sigma}\right]$. The number of Lorentz indices now does not match among the different terms. 
- It is unclear how to obtain the self-dual $H_{\mu \nu \rho}$ field strength from a Lagrangian. This self-duality would have to be imposed by hand, as we did for the action in the tensor branch.

- The instanton strings become tensionless at the conformal fixed point. This will then cause the vacuum of the theory to consist of wild fluctuations of strongly coupled tensionless instanton strings. This is in line with the first observation, that we would not expect there to be a vacuum around which we can perform an expansion.

These points seem to suggest that these theories cannot be approximated in terms of quantum fluctuations on top of a classical Lagrangian description. The theory at the conformal fixed point is inherently strongly coupled, causing all quantum fluctuations to be equally relevant.

In this sense these $6 \mathrm{~d} \mathcal{N}=(1,0)$ theories thus differ from e.g. $\mathcal{N}=4 \mathrm{SYM}$, as the latter is conformal for any value of the coupling constant. The former however always have a coupling constant for the vector multiplet that is infinite at the conformal fixed point.

\subsection{Holographic $\mathrm{AdS}_{7}$ Description}

We will now discuss in more detail the the holographic description of the 6d SCFTs (earlier mentioned in section 3.6) that appear when we move to the origin of the tensor branch. This description was developed in a set of papers, most notably [75]-[74]. We adopt the notation of [74].

The six dimensional SCFTs have an $O S p(2,6 \mid 2)$ symmetry group, with $S O(2,6) \times S U(2)_{R}$ bosonic symmetries, see for example [130]. These symmetries are realised as the isometries of a Massive Type IIA background of the form,

$$
\begin{aligned}
& d s^{2}=f_{1}(z) d s_{A d S_{7}}^{2}+f_{2}(z) d z^{2}+f_{3}(z) d \Omega_{2}^{2}(\chi, \xi), \\
& B_{2}=f_{4}(z) \operatorname{Vol}_{\Omega_{2}}, \quad F_{2}=f_{5}(z) \operatorname{Vol}_{\Omega_{2}}, \quad e^{\phi}=f_{6}(z), \quad F_{0}=F_{0}(z),
\end{aligned}
$$

where we have defined $d \Omega_{2}^{2}(\chi, \xi)=d \chi^{2}+\sin ^{2} \chi d \xi^{2}$ and $\operatorname{Vol}_{\Omega_{2}}=\sin \chi d \chi \wedge d \xi$.

If we impose that $\mathcal{N}=(1,0)$ SUSY is preserved by the background, we need the functions $f_{i}(z)$ to satisfy some first order nonlinear BPS equations. These BPS equations are solved if the functions $f_{i}(z)$ in eq.(4.6) are all defined in terms of a function $\alpha(z)$ and its derivatives - see [124]-[74] for the details,

$$
\begin{aligned}
& f_{1}(z)=8 \sqrt{2} \pi \sqrt{-\frac{\alpha}{\alpha^{\prime \prime}}}, \quad f_{2}(z)=\sqrt{2} \pi \sqrt{-\frac{\alpha^{\prime \prime}}{\alpha}}, \quad f_{3}(z)=\sqrt{2} \pi \sqrt{-\frac{\alpha^{\prime \prime}}{\alpha}}\left(\frac{\alpha^{2}}{\alpha^{2}-2 \alpha \alpha^{\prime \prime}}\right), \\
& f_{4}(z)=\pi\left(-z+\frac{\alpha \alpha^{\prime}}{\alpha^{\prime 2}-2 \alpha \alpha^{\prime \prime}}\right), \quad f_{5}(z)=\left(\frac{\alpha^{\prime \prime}}{162 \pi^{2}}+\frac{\pi F_{0} \alpha \alpha^{\prime}}{\alpha^{\prime 2}-2 \alpha \alpha^{\prime \prime}}\right), \\
& f_{6}(z)=2^{\frac{5}{4}} \pi^{\frac{5}{2}} 3^{4} \frac{\left(-\alpha / \alpha^{\prime \prime}\right)^{\frac{3}{4}}}{\sqrt{\alpha^{\prime 2}-2 \alpha \alpha^{\prime \prime}}} .
\end{aligned}
$$


Where $\alpha(z)$ has to satisfy the differential equation

$$
\alpha^{\prime \prime \prime}=-162 \pi^{3} F_{0} .
$$

The function $\alpha(z)$ must be piece-wise continuous, this implies that $F_{0}$ can be piece-wise constant and discontinuous. The points where $F_{0}$ is discontinuous are the points where the flavour branes are located. These flavour branes backreact on the geometry as we explained earlier near the end of section 3.5.

The internal space $\mathcal{M}_{3}=\left(z, \Omega_{2}\right)$ is a two-sphere fibered over the $z$-interval. The warp factor $f_{3}(z)$ must vanish at the beginning and at the end of the $z$-interval $\left(z=0\right.$ and $z=z_{f}$ by convention), in such a way that the two-sphere shrinks smoothly at those points.

For a piece-wise constant and possibly discontinuous $F_{0}(z)$, the general solution to eq.(4.8) in each interval of constant $F_{0}$ is,

$$
\alpha(z)=a_{0}+a_{1} z+\frac{a_{2}}{2} z^{2}-\frac{162 \pi^{3} F_{0}}{6} z^{3} .
$$

As we observed above, the function $\alpha(z)$ is in general piece-wise continuous and generically a polynomial solution like the one above should be proposed for each interval $\left[z_{i}, z_{i+1}\right]$.

Imposing that the two-sphere shrinks smoothly at $z=0$ and $z=z_{f}$ implies that $\alpha(0)=$ $\alpha\left(z_{f}\right)=0$. We shall discuss various explicit solution in detail further in the text. Before that, let us first find general expressions for the brane-charges associated with the backgrounds in eq. (4.6).

\subsubsection{Page charges}

We define the conserved Page charges,

$$
\begin{aligned}
Q_{D p} & =\frac{1}{(2 \pi)^{7-p} g_{s}\left(\alpha^{\prime}\right)^{\frac{(7-p)}{2}}} \int F_{8-p}-B_{2} \wedge F_{6-p}, \\
Q_{N S 5} & =\frac{1}{4 \pi^{2} g_{s}^{2} \alpha^{\prime}} \int H_{3} .
\end{aligned}
$$

In what follows we set $g_{s}=\alpha^{\prime}=1$. Calculating explicitly for the NS5-brane charge. Using that $\alpha(0)=\alpha\left(z_{f}\right)=0$ we find,

$$
Q_{N S 5}=\frac{1}{4 \pi^{2}} \int_{z, \Omega_{2}} d z \partial_{z} f_{4}=\frac{1}{\pi} \int_{z=0}^{z=z_{f}} d z \partial_{z} f_{4}=\frac{f_{4}\left(z_{f}\right)-f_{4}(0)}{\pi}=-z_{f} .
$$

Up to an orientation-related sign, the size of the $z$-interval equals the number of fivebranes. Hence we choose $z_{f}$ to be a positive integer. We shall take $Q_{N S 5}=z_{f}=N_{5}$ in what follows. 
Calculating the charge of D6-branes, we find

$$
Q_{D 6}=\frac{1}{2 \pi} \int_{(\chi, \xi)} F_{2}-F_{0} B_{2}=\left[\frac{\alpha^{\prime \prime}+162 \pi^{3} F_{0} z}{81 \pi^{2}}\right]=\frac{\alpha^{\prime \prime}-z \alpha^{\prime \prime \prime}}{81 \pi^{2}} .
$$

The charge in eq.(4.12) computes the charge of D6-branes but also includes the charge of D6brane induced on the D8-branes. To avoid this 'overcounting', note that we can perform a large gauge transformation in any interval $[k, k+1]$ such that,

$$
\hat{B}_{2} \rightarrow B_{2}+k \pi d \Omega_{2} \text {. }
$$

We then find that in the interval $[k, k+1]$ the Page charge reads,

$$
Q_{D 6}=\frac{1}{2 \pi} \int_{S^{2}} F_{2}-F_{0} \hat{B}_{2}=\frac{1}{2 \pi} \times \frac{\alpha^{\prime \prime}-\alpha^{\prime \prime \prime}(z-k)}{162 \pi^{2}} \times 4 \pi .
$$

Using that on the $[k, k+1]$ interval the function $\alpha^{\prime \prime}(z)=-81 \pi^{2}\left[N_{k}+\left(N_{k+1}-N_{k}\right)(z-k)\right]$, we find that

$$
N_{D 6}=\frac{1}{2 \pi} \times \frac{\alpha^{\prime \prime}-\alpha^{\prime \prime \prime}(z-k)}{162 \pi^{2}} \times 4 \pi=-N_{k} .
$$

The sign can be attributed to a choice of orientation for the two-sphere. The expression above indicates that in the $[k, k+1]$ interval, there are $N_{k}$ D6-branes. Notice that the expression in eq.(4.12) also counts the charge of D6's induced on the D8's. We are subtracting these, by performing the large gauge transformation above.

We thus find that the number of only the D6-branes in the associated Hanany-Witten set-up is given by,

$$
N_{D 6}=-\frac{1}{81 \pi^{2}} \int_{0}^{z_{f}} \alpha^{\prime \prime}(z) d z
$$

This can be verified by explicitly performing this integral for a generic function $\alpha^{\prime \prime}(z)$, observing that it counts the sum of the ranks of the gauge groups. On each interval $[k, k+1]$ this gives,

$$
-\frac{1}{81 \pi^{2}} \int_{k}^{k+1} \alpha^{\prime \prime} d z=-\int_{k}^{k+1}\left[N_{k}+\left(N_{k+1}-N_{k}\right)(z-k)\right] d z=\frac{N_{k}+N_{k+1}}{2} .
$$

Summing over all the intervals (using that $N_{0}=N_{P+1}=0$ ), gives the total quantity,

$$
N_{D 6}=\sum_{k=0}^{P} \frac{N_{k}+N_{k+1}}{2}=N_{1}+N_{2}+\ldots .+N_{P}
$$

We also present an expression that calculates the number of D8-branes in any given HananyWitten set-up. Our proposed expression reads,

$$
N_{D 8}=\frac{1}{81 \pi^{2}}\left[\alpha^{\prime \prime \prime}(0)-\alpha^{\prime \prime \prime}\left(z_{f}\right)\right] \text {. }
$$


In other words, the jumps in $\alpha^{\prime \prime \prime}(z)$ across any interval counts D8-branes according to eq.(4.8). Adding these jumps leads to eq.(4.19).

These expressions are analogous to those derived in [135], for the case of Hanany-Witten setups associated with four dimensional $\mathcal{N}=2$ SCFTs. In Section 4.3 we test the new expressions in eqs.(4.16),(4.19) on some examples.

\subsubsection{Linking numbers}

An interesting quantity characterising of Hanany-Witten set-ups are the linking numbers. For the case at hand, with D6, D8 and NS5-branes these topological invariants (unchanged under Hanany-Witten moves) are defined for the j-th D8-brane $\left(L_{j}\right.$ is the linking number) and the i-th Neveu-Schwarz fivebrane ( $K_{i}$ being the corresponding linking number) by counting the number of the other branes to the left and right. More precisely, we have

$$
\begin{aligned}
& L_{j}=N_{D 6}^{\text {right }}-N_{D 6}^{\text {left }}+N_{N S}^{\text {left }}, \\
& K_{i}=N_{D 6}^{\text {right }}-N_{D 6}^{\text {left }}-N_{D 8}^{\text {right }} .
\end{aligned}
$$

They must satisfy a consistency relation

$$
\sum_{j=1}^{N_{8}} L_{j}+\sum_{i=1}^{N 5} K_{i}=0
$$

In the Hanany-Witten set-ups that are relevant for the SCFTs we study in this paper, all the linking numbers for the different Neveu-Schwarz fivebranes are equal. We have found that they can be holographically calculated by very simple expressions. Our proposal is that for these CFTs we calculate the linking numbers as,

$$
\begin{aligned}
& K_{1}=K_{2}=\ldots=K_{N 5}=\frac{1}{81 \pi^{2}} \alpha^{\prime \prime \prime}\left(z_{f}\right) \rightarrow \sum_{i=1}^{N_{5}} K_{i}=\frac{1}{81 \pi^{2}} \alpha^{\prime \prime \prime}\left(z_{f}\right) z_{f} . \\
& L_{i}=z_{i} \rightarrow \sum_{j=1}^{N_{8}} L_{j}=-\frac{1}{81 \pi^{2}} \alpha^{\prime \prime \prime}\left(z_{f}\right) z_{f} .
\end{aligned}
$$

These expressions satisfy eq.(4.21) and are analogous to those presented in the case of four dimensional CFTs with eight supercharges [135]. In Section 4.3 we test these expressions in a couple of examples. The reader is invited to apply the expressions of eqs.(4.16),(4.19),(4.22),(4.23) to the examples of the paper [136]. 
IV. $\mathrm{ADS}_{7}$ DUALS OF $6 \mathrm{D} \mathcal{N}=(1,0)$ SCFTS

\subsection{Connecting the holographic background with the CFT}

We briefly introduced the connection between the quiver SCFT and the AdS geometries (4.6) earlier in section 3.5. We will now illustrate this in more detail for the $6 \mathrm{~d}$ case and provide various examples.

The problem can be organised as follows: first, we consider a non-anomalous quiver with bifundamental matter, gauge and flavour groups satisfying the relation in eq.(4.5). Then, we define the rank function $R(z)$, a piecewise continuous linear function such that at $z=j$ (with $j$ being a positive integer number) the value $R(j)=N_{j}$ is the rank of the $j$-th gauge group. It was shown in [74] that this rank-function must be convex to satisfy the anomaly cancellation condition in eq.(4.5). The link with the holographic description for the $\mathrm{AdS}_{7}$ case is given by the identification,

$$
R(z)=-\frac{1}{81 \pi^{2}} \alpha^{\prime \prime}(z)
$$

Finally, after this identification, we need to determine the function $\alpha(z)$ by imposing boundary conditions and continuity of $\alpha$ and $\alpha^{\prime}$.

Working out examples is possibly the clearest way to explain the procedure to the reader not acquainted with this formalism. We first present full details for a simple example and then we consider a more generic situation. The interested reader can consult the examples in Section 2.1.1 of the paper [136].

\subsubsection{Example 1}

Consider the Hanany-Witten set-up, quiver and rank function $R(z)$ in Figures 4.1-4.2b.

In this example, the rank function and the function $\alpha^{\prime \prime}(z)$ are given by,

$$
R(z)=-\frac{1}{81 \pi^{2}} \alpha^{\prime \prime}(z)=N \begin{cases}z & 0 \leq z \leq 1 \\ (z-1)+1 & 1 \leq z \leq 2 \\ (z-2)+2 & 2 \leq z \leq 3 \\ 3 & 3 \leq z \leq 4 \\ 3-3(z-4) & 4 \leq z \leq 5 .\end{cases}
$$

\footnotetext{
${ }^{1}$ In order for the background to capture faithfully the CFT dynamics one should work with long linear quivers and with large ranks. In this sense the examples of eqs.(2.6) and (2.8) of [136] are rigorously trustable. Our examples in this section should be taken as illustrative of the procedure.
} 


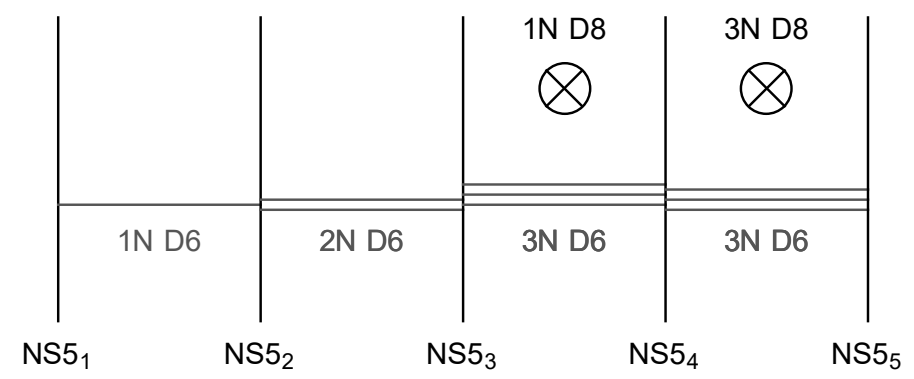

Figure 4.1: The Hanany-Witten set-up for the field theory. The vertical lines denote individual Neveu-Schwarz branes extended on the $\left(x_{4}, x_{5}\right)$ space. The horizontal ones D6-branes, that extend on $x_{6}$, in between fivebranes. The crossed-circles represent D8-branes, that extend on the $\left(x_{7}, x_{8}, x_{9}\right)$ directions. All the branes share the Minkowski directions. This realises the isometries $S O(1,5) \times S O(3)$.

This implies that the generic function $\alpha(z)$ for this example is,

$$
\alpha(z)=-81 \pi^{2} N \begin{cases}a_{0}+a_{1} z+\frac{z^{3}}{6} & 0 \leq z \leq 1 \\ b_{0}+b_{1}(z-1)+\frac{1}{2}(z-1)^{2}+\frac{1}{6}(z-1)^{3} & 1 \leq z \leq 2 \\ c_{0}+c_{1}(z-2)+2 \frac{(z-2)^{2}}{2}+\frac{1}{6}(z-2)^{3} & 2 \leq z \leq 3 \\ d_{0}+d_{1}(z-3)+3 \frac{(z-3)^{2}}{2} & 3 \leq z \leq 4 \\ p_{0}+p_{1}(z-4)+3 \frac{(z-4)^{2}}{2}-3 \frac{(z-4)^{3}}{6} & 4 \leq z \leq 5 .\end{cases}
$$

To determine the ten integration constants, we need to impose:

- That $\alpha(0)=\alpha(5)=0$. This is to have an internal space that shrinks smoothly at the beginning and end of the $z$-interval. These conditions imply

$$
a_{0}=0, \quad p_{0}+p_{1}+\frac{3}{2}-\frac{3}{6}=0
$$

- That the function $\alpha(z)$ is continuous, this implies the equations,

$$
\begin{array}{cc}
a_{1}+\frac{1}{6}=b_{0}, & b_{0}+b_{1}+\frac{1}{2}+\frac{1}{6}=c_{0}, \\
c_{0}+c_{1}+1+\frac{1}{6}=d_{0}, & d_{0}+d_{1}+\frac{3}{2}=p_{0} .
\end{array}
$$

- That the function $\alpha^{\prime}(z)$ is continuous. This implies,

$$
a_{1}+\frac{1}{2}=b_{1}, \quad b_{1}+1+\frac{1}{2}=c_{1}, \quad c_{1}+2+\frac{1}{2}=d_{1}, \quad d_{1}+3=p_{1} .
$$




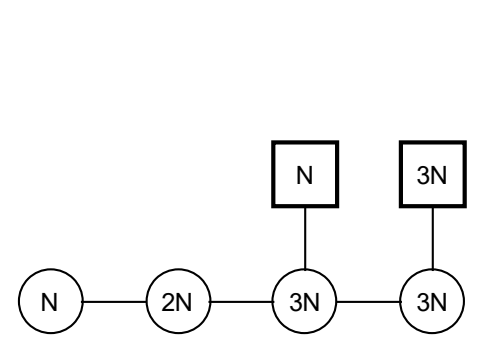

(a)

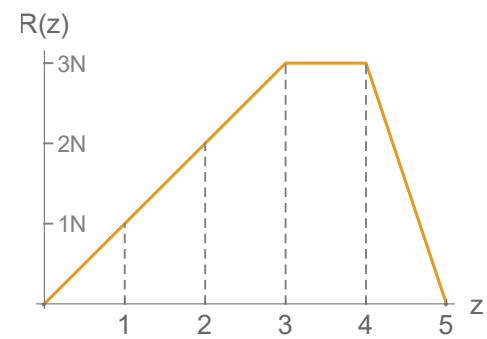

(b)

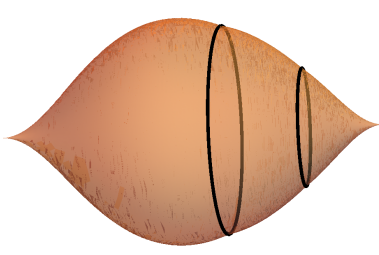

(c)

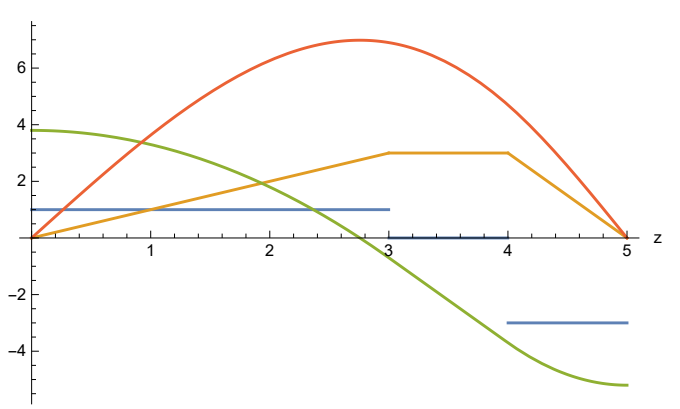

(d)

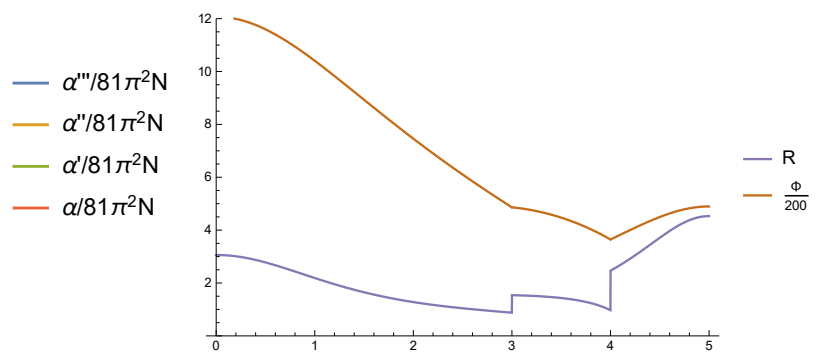

(e)

Figure 4.2: (a) The quiver corresponding to the Hanany-Witten set-up above. (b) The rank function $R(z)$ corresponding to the field theory. (c) Illustration of the $S^{2}$ fibered along the $z$-direction with the locations of the flavour branes shown in black. (d) The corresponding function $\alpha(z)$ and its derivatives. (e) Ricci scalar and dilaton for the corresponding geometry.

Solving these equations we find,

$$
\begin{aligned}
& a_{0}=0, \quad-5 a_{1}=19, \quad-30 b_{0}=109, \quad-10 b_{1}=33, \quad-15 c_{0}=94, \quad-5 c_{1}=9, \\
& -10 d_{0}=69, \quad 10 d_{1}=7, \quad-10 p_{0}=47, \quad 10 p_{1}=37 .
\end{aligned}
$$

In this way, we have a well defined function $\alpha(z)$.

We can apply our expressions for the number of NS, D6 and D8-branes and linking numbers. Using eqs.(4.11), (4.16) and (4.19) we find,

$$
\begin{aligned}
& N_{N S 5}=z_{f}=5, \quad N_{D 8}=\frac{1}{81 \pi^{2}}\left[\alpha^{\prime \prime \prime}(0)-\alpha^{\prime \prime \prime}\left(z_{f}\right)\right]=4 N \\
& N_{D 6}=-\frac{1}{81 \pi^{2}} \int_{0}^{z_{f}} \alpha^{\prime \prime}(z) d z=9 N .
\end{aligned}
$$

Notice that this coincides with the numbers we count from the Hanany-Witten set-up in Figure 4.1. 
We can also calculate the linking numbers using eqs.(4.22), (4.23). We find,

$$
\begin{aligned}
K_{1}= & K_{2}=\ldots=K_{5}=\frac{1}{81 \pi^{2}} \alpha^{\prime \prime \prime}\left(z_{f}\right)=-3 N \\
& \rightarrow \sum_{i=1}^{N_{5}} K_{i}=-15 N . \\
L_{1}= & L_{2}=\ldots=L_{N}=3, \quad \hat{L}_{1}=\ldots=\hat{L}_{3 N}=4 \\
& \rightarrow \sum_{i=1}^{N_{8}} L_{i}+\hat{L}_{i}=3 N+4 \times 3 N=15 N .
\end{aligned}
$$

We have denoted by $L_{i}, \hat{L}_{i}$ the linking numbers of the D8-branes in the first and second stacks. These numbers are also obtained by simple inspection of the Hanany-Witten diagram. The relation in eq.(4.21) is satisfied. The entanglement entropy can be calculated straightforwardly using eq.(6.60).

\subsubsection{Example 2}

Let us consider the quiver depicted in Figure 4.3, with three gauge groups $S U(N) \times S U(2 N) \times$ $S U(3 N)$ ending with a flavour group $S U(4 N)$. Notice that each node satisfies the anomaly cancellation condition of eq.(4.5).

The function $R(z)$ describing the ranks of this quiver is

$$
R_{1}(z)=N \begin{cases}z & 0 \leq z \leq 3 \\ 12-3 z & 3 \leq z \leq 4\end{cases}
$$

indicating the presence of gauge groups $S U(N)$ at $z=1, S U(2 N)$ at $z=2$ and $S U(3 N)$ at $z=3$. In this sense, the $z$-direction of the supergravity background 'encodes' the field theory information. The slope of the first three nodes is $s=N$, which translates to $a_{3}=b_{3}=c_{3}=N$. Similarly $p_{3}=-3 N$. The change in slope $\Delta s=-4 N$ indicates the presence of the $S U(4 N)$ flavour group. On the other hand, in the first interval $0 \leq z \leq 1$, there is no gauge group, hence $a_{2}=0$, while the gauge group in the second interval is $S U(N)$, indicating that $b_{2}=1$. Similarly $c_{2}=2$ and $p_{2}=3$, reflecting the presence of the $S U(2 N)$ and $S U(3 N)$ gauge groups. With this, we can write,

$$
\alpha(z)=-81 \pi^{2} N \begin{cases}a_{1} z+\frac{1}{6} z^{3} & 0 \leq z \leq 1 \\ b_{0}+b_{1}(z-1)+\frac{1}{2}(z-1)^{2}+\frac{1}{6}(z-1)^{3} & 1 \leq z \leq 2 \\ c_{0}+c_{1}(z-2)+\frac{2}{2}(z-2)^{2}+\frac{1}{6}(z-2)^{3} & 2 \leq z \leq 3 \\ p_{0}+p_{1}(z-3)+\frac{3}{2}(z-3)^{2}-\frac{1}{2}(z-3)^{3} & 3 \leq z \leq 4\end{cases}
$$




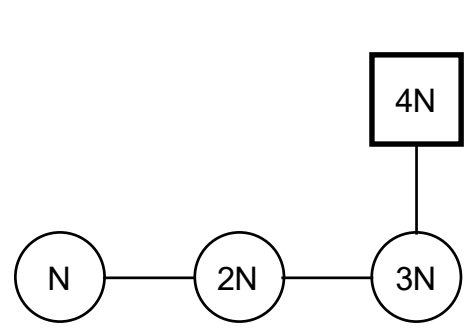

(a)

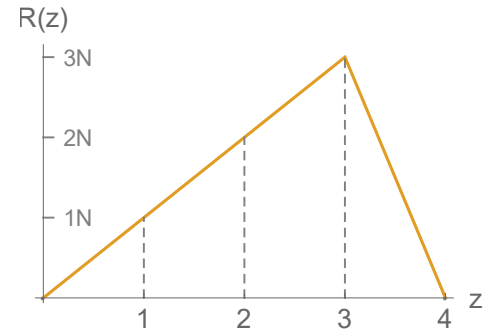

(b)

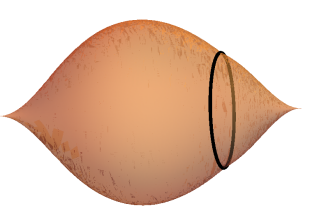

(c)

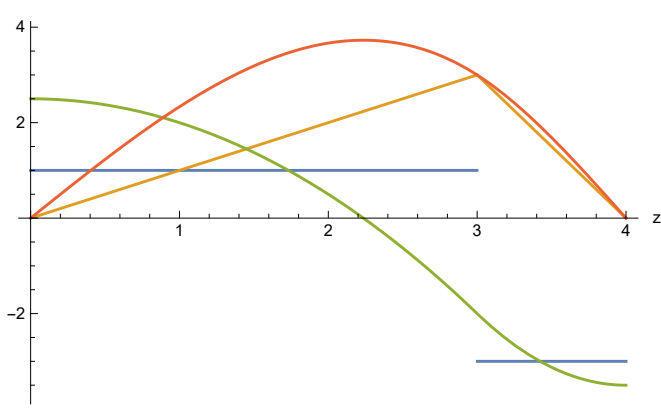

(d)

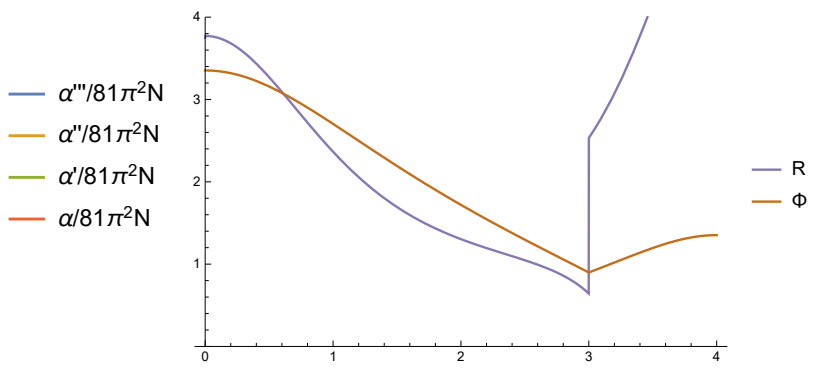

(e)

Figure 4.3: (a) The quiver encoding the dynamics of our example CFT. (b) The rank function $R(z)$ corresponding to the field theory. (c) Illustration of the $S^{2}$ fibered along the $z$-direction with the locations of the flavour branes shown in black. (d) The corresponding function $\alpha(z)$ and its derivatives. (e) Ricci scalar and dilaton for the corresponding geometry.

where the remaining constants are determined by imposing continuity of $\alpha, \alpha^{\prime}$ and that $\alpha(z=$ $4)=0$. This gives,

$$
a_{1}=-\frac{5}{2}, \quad b_{1}=-2, \quad c_{1}=-\frac{1}{2}, \quad p_{1}=2 ; \quad b_{0}=-\frac{7}{3}, \quad c_{0}=-\frac{11}{3}, \quad p_{0}=-3 .
$$

The function $\alpha(z)$ describing the background in eq.(4.6), dual to the quiver CFT in Figure 4.3 reads

$$
\alpha_{1}(z)=-81 \pi^{2} N \begin{cases}-\frac{5}{2} z+\frac{1}{6} z^{3} & 0 \leq z \leq 1 \\ -\frac{7}{3}-2(z-1)+\frac{1}{2}(z-1)^{2}+\frac{1}{6}(z-1)^{3} & 1 \leq z \leq 2 \\ -\frac{11}{3}-\frac{1}{2}(z-2)+\frac{2}{2}(z-2)^{2}+\frac{1}{6}(z-2)^{3} & 2 \leq z \leq 3 \\ -3+2(z-3)+\frac{3}{2}(z-3)^{2}-\frac{1}{2}(z-3)^{3} & 3 \leq z \leq 4\end{cases}
$$

We have worked with a quiver with three colour nodes and one flavour node. Strictly speaking, the supergravity description is valid if the number of colour nodes is taken to be large [74]. Our 
example in eq.(4.27) illustrates the procedure. In order to have a better holographic description of the CFT, we should work with a quiver with $S U(N) \times S U(2 N) \times S U(3 N) \times S U(4 N) \times \ldots . \times$ $S U(P N)$ closed by an $S U(P N+N)$-flavour group (and taking $P$ to be large). In that case, we write the function,

$$
\begin{aligned}
& -\frac{\alpha_{1}(z)}{81 \pi^{2} N}= \\
& \begin{cases}a_{1} z+\frac{1}{6} z^{3} & 0 \leq z \leq 1 \\
\left(k a_{1}+\frac{k^{3}}{6}\right)+\left(a_{1}+\frac{k^{2}}{2}\right)(z-k)+\frac{k}{2}(z-k)^{2}+\frac{1}{6}(z-k)^{3} & k \leq z \leq(k+1), \\
\left(P a_{1}+\frac{P^{3}}{6}\right)+\left(a_{1}+\frac{P^{2}}{2}\right)(z-P)+\frac{P}{2}(z-P)^{2}-\frac{P}{6}(z-P)^{3} & P \leq z \leq P+1,\end{cases}
\end{aligned}
$$

where

$$
k=1, \ldots, P-1, \quad-6 a_{1}=P^{2}+2 P .
$$

The case of a quiver with increasing ranks, not closed by the flavour group, is described holographically by the function $\alpha_{1}(z)=-81 \pi^{2} N\left(a_{1} z+\frac{z^{3}}{6}\right)$, being $a_{1}$ a free parameter.

It is instructive to plot the function $-\frac{\alpha_{1}(z)}{81 \pi^{2}}$ and its derivatives for the background defined by eq.(4.27), see Figure 4.3d. We also plot the fields defining the background and the Ricci scalar. None of these functions are divergent for the $\alpha(z)$ in eq.(4.27). We shall use this background in a later chapter as an example to study the dynamics of a string configuration that rotates and winds on it.

\subsubsection{Example 3}

As a second example, we can work out the function $\alpha(z)$ for the quiver in Figure 4.4a. This quiver starts with an $S U(N)$-flavour node followed by three nodes $S U(N)$-colour, and it is closed by a final $S U(N)$-flavour node. The function describing the ranks is,

$$
R_{2}(z)=N \begin{cases}z & 0 \leq z \leq 1 \\ 1 & 1 \leq z \leq 3 \\ 4-z & 3 \leq z \leq 4\end{cases}
$$

and the function that determines the holographic description $\alpha_{2}(z)$ is,

$$
\alpha_{2}(z)=-81 \pi^{2} N \begin{cases}-\frac{3}{2} z+\frac{1}{6} z^{3} & 0 \leq z \leq 1 \\ -\frac{4}{3}-(z-1)+\frac{1}{2}(z-1)^{2} & 1 \leq z \leq 2 \\ -\frac{11}{6}+\frac{1}{2}(z-2)^{2} & 2 \leq z \leq 3 \\ -\frac{4}{3}+(z-3)+\frac{1}{2}(z-3)^{2}-\frac{1}{6}(z-3)^{3} & 3 \leq z \leq 4\end{cases}
$$

The holographic description of this CFT is valid when the number of nodes is large. We take the above quiver to be long enough for the illustrative purposes we aim at. 


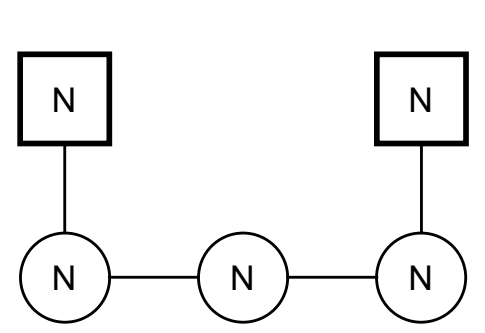

(a)

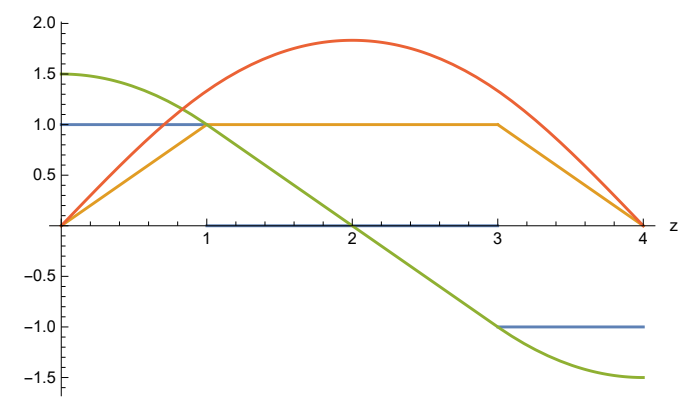

(d)

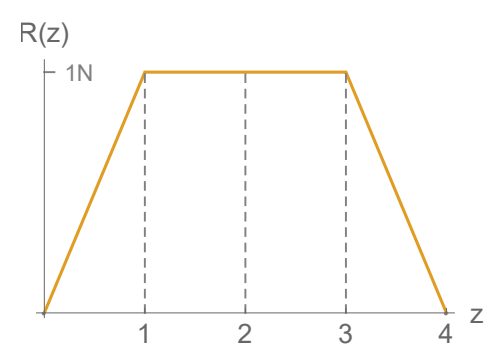

(b)

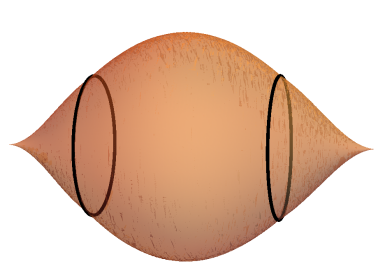

(c)

Figure 4.4: (a) The quiver encoding the dynamics of our this example. (b) The rank function $R(z)$ corresponding to the field theory. (c) Illustration of the $S^{2}$ fibered along the $z$-direction with the locations of the flavour branes shown in black. (d) The corresponding function $\alpha(z)$ and its derivatives. (e) Ricci scalar and dilaton for the corresponding geometry.

\section{An Endless Quiver}

Finally, we shall consider an endless quiver. The quiver starts with an $S U(N)$-flavour group and is continued by an infinite tail of $S U(N)$-colour groups. As a consequence, the $z$-coordinate is unbounded. There is one integration constant that remains undetermined. The function describing the ranks is

$$
R_{3}(z)=N \begin{cases}z & 0 \leq z \leq 1 \\ 1 & 1 \leq z \leq \infty\end{cases}
$$


The function $\alpha_{3}(z)$ reads,

$$
\begin{aligned}
& -\frac{\alpha_{1}(z)}{81 \pi^{2} N}= \\
& \begin{cases}a_{1} z+\frac{1}{6} z^{3} & 0 \leq z \leq 1 \\
\left(a_{1}+\frac{1}{6}\right)+\left(a_{1}+\frac{1}{2}\right)(z-1)+\frac{1}{2}(z-1)^{2} & 1 \leq z \leq 2 \\
\left(2 a_{1}+\frac{7}{6}\right)+\left(a_{1}+\frac{3}{2}\right)(z-2)+\frac{1}{2}(z-2)^{2} & 2 \leq z \leq 3 \\
\left(3 a_{1}+\frac{19}{6}\right)+\left(a_{1}+\frac{5}{2}\right)(z-3)+\frac{1}{2}(z-3)^{2} & 3 \leq z \leq 4 \\
\left(4 a_{1}+\frac{37}{6}\right)+\left(a_{1}+\frac{7}{2}\right)(z-4)+\frac{1}{2}(z-4)^{2} & 4 \leq z \leq 5 \\
\cdots & \\
\left(P a_{1}+\frac{3 P^{2}-3 P+1}{6}\right)+\left(a_{1}+\frac{2 P-1}{2}\right)(z-P)+\frac{1}{2}(z-P)^{2} & P \leq z \leq P+1 \\
\cdots \cdots & \end{cases}
\end{aligned}
$$

Ending the quiver with a flavour $S U(P+1)$ node, reflects in a cap-off of the geometry at $z=P+1$. This is achieved by adding a term $-\frac{1}{6}(z-P)^{3}$ to the last line and choosing $a_{1}=-\frac{P}{2}$. This would correspond to the quiver in Figure 4.5.

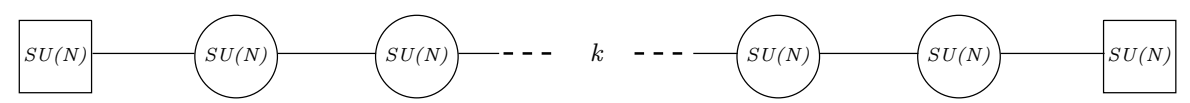

Figure 4.5: The quiver encoding the dynamics of the third example CFT. The long tail of colour $S U(N)$ ends with a flavour group.

\subsubsection{Most generic example}

Following the same logic, let us consider the most general quiver field theory. The gauge group is

$$
S U\left(N_{1}\right) \times S U\left(N_{2}\right) \times \ldots \times S U\left(N_{P}\right) .
$$

The flavour group, with each element associated with a colour node, is

$$
S U\left(F_{1}\right) \times S U\left(F_{2}\right) \times \ldots \times S U\left(F_{P}\right) .
$$

The consistency condition for the CFTs is,

$$
2 N_{k}-N_{k+1}-N_{k-1}=F_{k}
$$




\section{IV. $\operatorname{ADS}_{7}$ DUALS OF $6 \mathrm{D} \mathcal{N}=(1,0)$ SCFTS}

We are using that $N_{-1}=N_{P+1}=0$. The rank function is,

$$
R(z)=\left\{\begin{array}{cc}
N_{1} z & 0 \leq z \leq 1 \\
N_{1}+\left(N_{2}-N_{1}\right)(z-1) & 1 \leq z \leq 2 \\
N_{2}+\left(N_{3}-N_{2}\right)(z-2) & 2 \leq z \leq 3 \\
N_{k}+\left(N_{k+1}-N_{k}\right)(z-k) & k \leq z \leq k+1, \quad k:=3, \ldots, P \\
N_{P}-N_{P}(z-P) & P \leq z \leq P+1 .
\end{array}\right.
$$

We can write from here the function $\alpha(z)$. Imposing that $\alpha(z=0)=0$, we find after some algebra

$$
\begin{aligned}
& -\frac{\alpha(z)}{81 \pi^{2}}= \\
& \left\{\begin{array}{cc}
b_{0} z+N_{1} \frac{z^{3}}{6} & 0 \leq z \leq 1 \\
a_{k}+b_{k}(z-k)+\frac{N_{k}}{2}(z-k)^{2}+\frac{\left(N_{k+1}-N_{k}\right)}{6}(z-k)^{3} & k \leq z \leq(k+1), \\
a_{p}+b_{p}(z-P)+\frac{N_{P}}{2}(z-P)^{2}-\frac{N_{P}}{6}(z-P)^{3} & P \leq z \leq P+1 .
\end{array}\right.
\end{aligned}
$$

To satisfy the conditions of continuity for $\alpha(z)$ and of $\alpha^{\prime}(z)$ and also imposing $\alpha(z=P+1)=0$ leads to the coefficients,

$$
\begin{aligned}
& b_{0}=-\frac{1}{(P+1)} \sum_{i=1}^{P}(P+1-i) N_{i}, \\
& a_{k}=k b_{0}+\frac{N_{k}}{6}+\sum_{i=1}^{k}(k-i) N_{i}, \quad k: 1, \ldots . P, \\
& b_{k}=b_{0}+\frac{N_{k}}{2}+\sum_{i=1}^{k-1} N_{i}, \quad k:=1, \ldots, P .
\end{aligned}
$$

From here we find the number of branes in the associated Hanany-Witten set-up. Using the expressions proposed earlier, we find

$$
\begin{aligned}
& N_{D 6}=-\frac{1}{81 \pi^{2}} \int_{0}^{P+1} \alpha^{\prime \prime}(z) d z=N_{1}+N_{2}+\ldots+N_{P}, \quad N_{N S 5}=P+1, \\
& N_{D 8}=\frac{1}{81 \pi^{2}}\left(\alpha^{\prime \prime \prime}(0)-\alpha^{\prime \prime \prime}(P+1)\right)=N_{1}+N_{P}=F_{1}+F_{2}+\ldots .+F_{P} .
\end{aligned}
$$

More interestingly, we can derive a general expression for the central charge of the quiver, using the expression derived in [136]. In fact, in general we have,

$$
c=-\frac{2^{8}}{3^{8} \times 16 \times G_{N}} \int_{0}^{P+1} \alpha^{\prime \prime}(z) \alpha(z) d z, \quad G_{N}=8 \pi^{6} .
$$


Evaluating this expression in general gives us

$$
\begin{aligned}
& -\frac{\pi^{2}}{2} c=\left[\frac{b_{0} N_{1}}{3}+\frac{N_{1}^{2}}{30}\right]+\left[\frac{a_{P} N_{P}}{2}+\frac{b_{P} N_{P}}{6}+\frac{N_{P}^{2}}{30}\right]+ \\
& +\frac{1}{30} \sum_{k=1}^{P-1}\left[N_{k}^{2}+3 N_{k} N_{k+1}+N_{k+1}^{2}+15 a_{k}\left(N_{k}+N_{k+1}\right)+5 b_{k}\left(N_{k}+2 N_{k+1}\right)\right] .
\end{aligned}
$$

\section{Two Cases}

We can test the expression in eq.(4.35) in two particularly simple cases:

First, in the case in which all the ranks are equal $N_{i}=N$. This needs of only two flavour groups, of rank $N$ at the beginning and at the end of the quiver. In this case, calculating the expressions in eqs.(4.32), (4.35), we find

$$
\begin{aligned}
& b_{0}=-\frac{N P}{2}, \quad a_{k}=\frac{N}{2}\left(-k P+k^{2}-k+\frac{1}{3}\right), \\
& b_{k}=\frac{N}{2}(2 k-P-1), \quad k: 1, \ldots, P . \\
& c=\frac{N^{2} P^{3}}{6 \pi^{2}}\left(1+\frac{3}{P}-\frac{1}{P^{2}}+\frac{1}{5 P^{3}}\right) \sim \frac{N^{2} P^{3}}{6 \pi^{2}} .
\end{aligned}
$$

This last result makes sense, because the supergravity approximation is good when $P \rightarrow \infty$, that is in the limit of long-linear quivers. In this case, the central charge calculated above precisely with that derived in [136] (see eq.(2.16) of that paper).

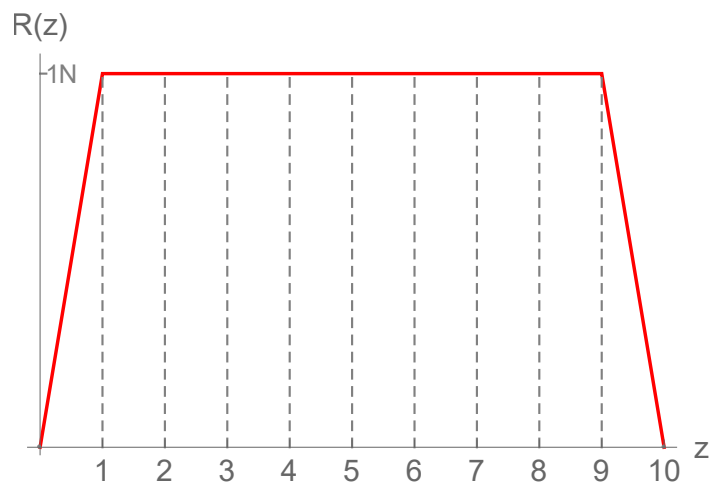

(a)

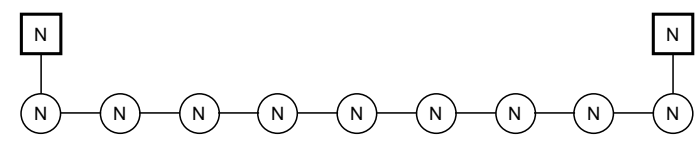

(b)

Figure 4.6: An example of the rank function (a) and quiver (b) for the first case with all the ranks equal $N_{i}=N$, and two flavour groups, of rank $N$ at the beginning and end of the quiver. 


\section{IV. $\operatorname{ADS}_{7}$ DUALS OF $6 \mathrm{D} \mathcal{N}=(1,0)$ SCFTS}

A second test is to consider the quiver in which the ranks increase as $N_{k}=k N$. This only needs of a flavour group, or rank $(P+1)$ attached to the last node of rank $P N$. The expressions in eqs.(4.32), (4.35), read in this case

$$
\begin{aligned}
& b_{0}=-\frac{N P}{6}(P+2), \quad a_{k}=\frac{k N}{6}\left(-2 P+k^{2}-P^{2}\right), \\
& b_{k}=\frac{N}{6}\left(3 k^{2}-P^{2}-2 P\right), \quad k: 1, \ldots, P . \\
& c=\frac{2 N^{2} P^{5}}{45 \pi^{2}}\left(1+\frac{5}{P}+\frac{5}{P^{2}}+\frac{1}{P^{3}}\right) \sim \frac{2 N^{2} P^{5}}{45 \pi^{2}} .
\end{aligned}
$$

In the limit of long-linear quivers the central charge calculated above precisely with that derived in [136], see eq.(2.15) in that paper.

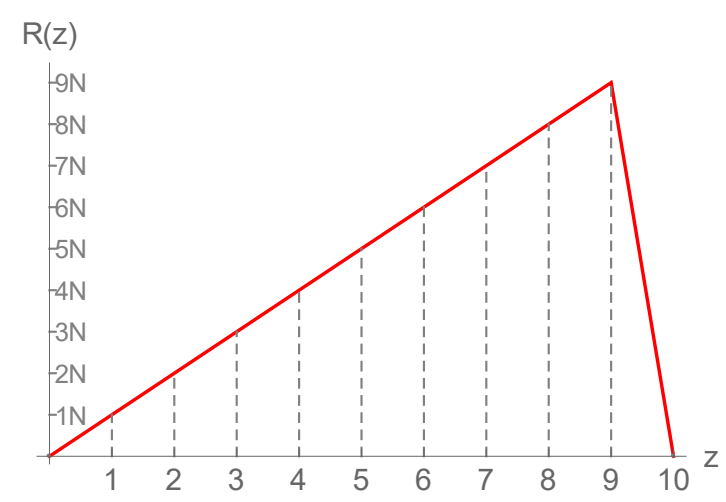

(a)

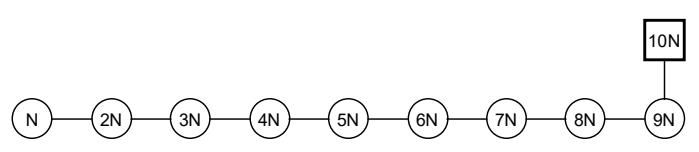

(b)

Figure 4.7: An example of the rank function (a) and quiver (b) for the second case with all the ranks linearly increasing as $N_{k}=k N$, and a flavour group of rank $(P+1)$ at the end of the quiver.

We have then good reasons to believe that the expression in eq.(4.35) is correct. 
V. (NON)-INTEGRABILE $6 \mathrm{D} \mathcal{N}=(1,0)$ SCFTS

\section{Chapter 5}

\section{(Non)-Integrabile $\mathbf{6 d} \mathcal{N}=(1,0)$ SCFTs}

\section{$5.1 \quad$ Introduction}

In chapter 2 we introduced the ideas of classical integrability on the string worldsheet. In general it is difficult to find a Lax connection and prove the string is integrable on a general background. Easier is however to show by means of a counterexample that the string cannot be integrable on a particular target space. This can be done by showing that a subsector of the string worldsheet is non-integrable. Since the integrability of the string worldsheet guarantees the integrability of all possible subsectors this then proves, by finding a counterexample, the total string worldsheet cannot be integrable.

In section 5.2 we obtain the equations of motion for classical string embeddings that wrap and move around certain directions on the $\mathrm{AdS}_{7}$ geometries we introduced in the previous chapter.

In section 5.3 we apply Kovacic's algorithm to these equations of motion, and try to prove the non-existence of Liouvillian solutions for these equations of motion. As we will explain in the introduction of this chapter, this would prove the non-integrability of the entire string worldsheet. Unfortunately this method is rather inconclusive when applied to the equations of motion we obtain in section 5.2.

To further supplement our analysis, we use numerical indicators of chaos in order to show the equations of motion on the various $\mathrm{AdS}_{7}$ backgrounds are indeed non-integrable. To this end we introduce our numerical methods in section 5.4.

In section 5.5 we apply these numerical methods to the various backgrounds we earlier examined using Kovacic's algorithm. Our conclusions are as follows:

- We find that the dynamics of strings on all $\mathrm{AdS}_{7}$ backgrounds corresponding to (finite) quivers with (a finite number of) flavour branes are non-integrable. 
- We suggest that strings on the $\mathrm{AdS}_{7}$ background corresponding to an infinite linear quiver with rank function $R(z) \sim z$ might be integrable. When we consider a finite linear quiver - by adding a single flavour group to the end - we again find clear evidence of non-integrability.

- For the massless solutions where $\alpha(z)=R^{2}-\mu z$, we find the strings can only be integrable in the limit $R \rightarrow \infty$, corresponding to an infinite constant quiver. When we consider finite values for $R$ the geometry has singular points at the end of the $z$-interval that now cause the string dynamics to again become non-integrable.

- At last, we consider a somewhat unusual solution, where $\alpha(z)=A \sin \omega z$. We find that this particular function $\alpha(z)$ corresponds to an integrable background! This background corresponds to one that has an infinite number of flavour branes. The details of this peculiar background, together with the corresponding quiver structure of this background will be the subject of further extensive study in chapter 6 .

\subsection{Classical Equations of Motion for String Embeddings}

We start our analysis by studying the dynamics of a classical bosonic string soliton, derived from the Polyakov action

$$
S_{P}=\frac{1}{4 \pi \alpha^{\prime}} \int_{\Sigma} d^{2} \sigma\left(\eta^{a b} G_{\mu \nu}+\epsilon^{a b} B_{\mu \nu}\right) \partial_{a} X^{\mu} \partial_{b} X^{\nu}
$$

supplemented by the Virasoro constraint $T_{a b}=0$, where

$$
T_{a b}=\partial_{a} X^{\mu} \partial_{b} X^{\nu} G_{\mu \nu}-\frac{1}{2} \eta_{a b} \eta^{c d} \partial_{c} X^{\mu} \partial_{d} X^{\nu} G_{\mu \nu}
$$

We will now focus our attention on a string soliton that moves on - and wraps itself around the angles of - both the $\operatorname{AdS}_{7}\left(t, \rho, \varphi, \theta_{i}\right)$-space and the internal $\mathcal{M}_{3}(z, \chi, \xi)$-manifold. This string soliton is parametrised by an embedding on this space that is as follows:

$$
\begin{array}{r}
t=t(\tau), \quad \rho=\rho(\tau), \quad \varphi=\varphi(\tau), \quad \theta=\lambda \sigma \\
z=z(\tau), \quad \chi=\chi(\tau), \quad \xi=\kappa \sigma .
\end{array}
$$

Here $\kappa$ and $\lambda$ indicate how many times the string wraps itself around the $\theta$ - and $\xi$-directions respectively. We write the $\mathrm{AdS}_{7}$-metric in global coordinates as

$$
d s_{\mathrm{AdS}_{7}}^{2}=-\cosh ^{2} \rho d t^{2}+d \rho^{2}+\sinh ^{2} \rho\left(d \varphi^{2}+\sin ^{2} \varphi d \theta^{2}+\cos ^{2} \varphi d \Omega_{S^{3}}^{2}\right),
$$


where $\varphi \in[0, \pi / 2]$ and $\theta \in[0,2 \pi]$. For the above string embedding we can write down an effective Lagrangian that will be of the form

$$
\begin{aligned}
\mathcal{L}=f_{1}(z)\left(\cosh ^{2} \rho \dot{t}^{2}-\dot{\rho}^{2}-\sinh ^{2} \rho\left(\dot{\varphi}^{2}-\lambda^{2} \sin ^{2} \varphi\right)\right)-\ldots \\
\ldots-f_{2}(z) \dot{z}^{2}-f_{3}(z)\left(\dot{\chi}^{2}-\kappa^{2} \sin ^{2} \chi\right)+2 f_{4}(z) \kappa \dot{\chi} \sin \chi
\end{aligned}
$$

varying this Lagrangian gives for the equations of motion

$$
\begin{aligned}
2 f_{1}(z) \dot{t} & =\frac{2 E}{\cosh ^{2} \rho} \\
2 f_{1}(z) \ddot{\rho} & =-2 \frac{E^{2}}{f_{1}(z)^{2}} \frac{\sinh \rho}{\cosh ^{3} \rho}+2 f_{1}(z) \sinh \rho \cosh \rho\left(\dot{\varphi}^{2}-\lambda^{2} \sin ^{2} \varphi\right)-2 f_{1}^{\prime}(z) \dot{\rho} \dot{z} \\
2 f_{1}(z) \ddot{\varphi}= & -2 f_{1}(z)\left(\frac{\cosh \rho}{\sinh \rho} \dot{\varphi} \dot{\rho}+\lambda^{2} \cos \varphi \sin \varphi\right)-f_{1}^{\prime}(z) \dot{z} \dot{\varphi} \\
2 f_{3}(z) \ddot{\chi}= & 2 \kappa f_{4}^{\prime}(z) \dot{z} \sin \chi-2 f_{3}^{\prime}(z) \dot{z} \dot{\chi} \sin \chi \cos \chi \\
2 f_{2}(z) \ddot{z}= & f_{1}^{\prime}(z)\left(-\frac{E^{2}}{f_{1}(z)^{2}} \cosh ^{-2} \rho+\dot{\rho}^{2}+\sinh ^{2} \rho\left(\dot{\varphi}^{2}-\lambda^{2} \sin ^{2} \varphi\right)\right)-f_{2}^{\prime}(z) \dot{z}^{2}+\ldots \\
& \ldots+f_{3}^{\prime}(z)\left(\dot{\chi}^{2}-\kappa^{2} \sin ^{2} \chi\right)-2 \kappa \dot{\chi} \sin \chi f_{4}^{\prime}(z)
\end{aligned}
$$

Here the dots indicate derivatives with respect to $\tau$ and the primes indicate derivatives with respect to $z$. Notice that when we set $\rho=\varphi=\lambda=0$ this system of equations reduces to the system that was studied in [136], where the string only moves on and wraps around the internal space.

The Virasoro constraint for the string is for this embedding of the form

$$
\begin{gathered}
2 T_{\tau \tau}=2 T_{\sigma \sigma}=f_{1}(z)\left(-\cosh ^{2} \rho \dot{t}^{2}+\dot{\rho}^{2}+\sinh ^{2} \rho\left(\dot{\varphi}^{2}+\lambda^{2} \sin ^{2} \varphi\right)\right)+\ldots \\
\ldots+f_{2}(z) \dot{z}^{2}+f_{3}(z)\left(\dot{\chi}^{2}+\kappa^{2} \sin ^{2} \chi\right) \\
T_{\sigma \tau}=0
\end{gathered}
$$

The equations of motion imply that $\partial_{\sigma} T_{\sigma \sigma}=\partial_{\tau} T_{\tau \tau}=0$, the string soliton will thus satisfy the Virasoro constraint $T_{\sigma \sigma}=T_{\tau \tau}$ by making an appropriate choice for the integration constant $E$.

\subsection{Analytical (non)-Integrability}

We will now study if the equations of motion for this string embedding eq.(5.7) are nonintegable. To this end we will follow [163], and apply Kovacic's algorithm to these equations of motion, to prove the equation of motion have no Liouvillian solutions. The results of this method are unfortunately rather inconclusive. We therefore suggest a reader not specifically interested in this particular analysis to move on to sections 5.4 and 5.5 where we study the 
same equations of motion using numerical methods, and obtain more conclusive results and nicer pictures.

If the full string worldsheet theory is integrable on a particular background, it will be possible for every subsector to move to action-angle variables $\left(\phi_{k}, I_{k}\right)$, where the resulting (sub)system has the same number of conserved quantities as coordinates in phase space. The system will exhibit regular orbits in phase space (in contrast to chaotic trajectories) in these coordinates, an one can solve $\phi_{k}=I_{k} t$. Since there is a canonical transformation $\left(x_{k}, p_{k}\right) \rightarrow\left(\phi_{k}, I_{k}\right)$ the functions $X_{k}(t)$ should be given by Liouvillian functions ${ }^{1}$ in any other frame that is not actionangle variables.

Here we will study the 'normal' variational equations (NVEs) for the equations of motion in eq.(5.7), that are obtained by solving them first for a particular variable (setting the other variables to zero), and then expanding around this solution in terms of the other 'orthogonal' variables. If these variational equations do not admit a Liouvillian solution, the full equations of motion will not admit a Liouvillian solution either.

To obtain these variational equations we first solve the equations of motion for $\ddot{z}(\tau)$ by first choosing the solutions

$$
\begin{aligned}
& \ddot{\varphi}(\tau)=\dot{\varphi}(\tau)=\varphi(\tau)=0 \\
& \ddot{\chi}(\tau)=\dot{\chi}(\tau)=\chi(\tau)=0 \\
& \ddot{\rho}(\tau)=\dot{\rho}(\tau)=\rho(\tau)=0
\end{aligned}
$$

for the other variables. When we insert these solutions in the above system of equations, the equation of motion for $\ddot{z}$ reduces to

$$
2 f_{2}(z) \ddot{z}=-\frac{f_{1}^{\prime}(z)}{f_{1}(z)^{2}} E^{2}-\dot{z}^{2} f_{2}^{\prime}(z)
$$

After inserting the expressions for the functions $f_{i}(z)$ in terms of $\alpha(z)$, the above equation for $\ddot{z}$ is of the form

$$
\ddot{z}-\frac{1}{2}\left(\frac{\alpha \alpha^{\prime \prime \prime}-\alpha^{\prime} \alpha^{\prime \prime}}{2 \alpha^{2}}\right) \frac{\alpha}{\alpha^{\prime \prime}}\left(\frac{E^{2}}{16 \pi^{2}}-\dot{z}^{2}\right)=0
$$

which has the solution

$$
z_{\text {sol }}(\tau)=\frac{E}{4 \pi} \tau
$$

\footnotetext{
${ }^{1}$ The 'elementary' functions are polynomials, rational functions, exponential functions, and their inverse (the trigonometric function can be written as complex exponentials). This set of elementary functions is closed under composition, arithmetic operations and differentiation, but not under integration. The Liouvillian functions are all possible functions that can be recursively defined in terms of the elementary functions or integrals thereof. The set of Liouvillian functions is therefore closed under composition, arithmetic operations, and both differentiation and integration.
} 


\section{V. (NON)-INTEGRABILE $6 \mathrm{D} \mathcal{N}=(1,0)$ SCFTS}

for all $\alpha(z)$. We can then obtain the NVE by inserting this solution for $z(\tau)$ and expanding around the solutions (5.9), taking either of the following expansions

$$
\begin{aligned}
& \rho(\tau)=0+\epsilon r(\tau) \\
& \varphi(\tau)=0+\epsilon f(\tau) \\
& \chi(\tau)=0+\epsilon x(\tau)
\end{aligned}
$$

We introduce the idea behind Kovacic's algorithm in the next section and show in the following sections how this can be applied to the different NVE equations thus obtained.

\subsubsection{Kovacic's Algorithm}

Here we briefly describe Kovacic's algorithm [182] for the non-existence of closed form Liouvillian solutions (solutions that can be expressed in terms of algebraic, exponentials, or trigonometric functions, and integrals of thereof) for differential equations of the form,

$$
f^{\prime \prime}(\tau)+\mathcal{B}(\tau) f^{\prime}(\tau)+\mathcal{A}(\tau) f(\tau)=0
$$

where $\mathcal{A}(\tau), \mathcal{B}(\tau)$ are complex rational functions. The algorithm of [182] provides a Liouvillian solution or shows there is none (in which case we refer to the differential equation (5.14) as non-integrable).

We will not describe the algorithm itself here, but limit ourselves to explain the logic behind Kovacic's algorithm and the resulting necessary but not-sufficient conditions that a combination the functions $\mathcal{A}, \mathcal{B}, \mathcal{B}^{\prime}$ must satisfy, for the eq.(5.14) to be Liouville-integrable.

We start by redefining the function $f(\tau)$ and rewriting the differential equation as,

$$
\begin{aligned}
& f(\tau)=e^{\frac{1}{2} \int B(\tau)} w, \\
& w^{\prime \prime}(\tau)=V(\tau) w(\tau), \quad V(\tau)=\frac{1}{4}\left(2 \mathcal{B}^{\prime}+\mathcal{B}^{2}-4 \mathcal{A}\right) .
\end{aligned}
$$

The Liouvillian (non-)integrability of the function $w(\tau)$ can then be translated to a statement about the poles of the 'potential' $V(\tau)$.

This results comes from differential Galois theory, where one studies the Galois group of invariances that act on the solutions of the differential equation. Suppose that $\zeta(\tau)$ and $\eta(\tau)$ are two solutions of the differential equation, another solution can be obtained as

$$
\left(\begin{array}{l}
\eta^{\prime} \\
\zeta^{\prime}
\end{array}\right)=\left(\begin{array}{ll}
a & b \\
c & d
\end{array}\right)\left(\begin{array}{l}
\eta \\
\zeta
\end{array}\right)
$$

for some $a, b, c, d \in \mathbb{C}$.

Kovacic showed that for the differential equation (5.15) the solutions can be invariant under four possible finite subgroups of $S L(2, C)$ : 
- Case 1: when the subgroup is generated by a triangular matrix of the form

$$
G=\left[\begin{array}{cc}
a & 0 \\
b & \frac{1}{a}
\end{array}\right]
$$

with $a, b$ complex numbers. In this case $w(\tau)$ is a rational function of degree 1.

- Case 2: when the subgroup of $S L(2, C)$ is generated by (off)-diagonal matrices of the form,

$$
G=\left[\begin{array}{cc}
c & 0 \\
0 & \frac{1}{c}
\end{array}\right], \quad G=\left[\begin{array}{cc}
0 & c \\
-\frac{1}{c} & 0
\end{array}\right]
$$

with $c$ a complex number. In this case the functions $w(\tau)$ is rational of degree 2

- Case 3: the subgroup $G$ is another finite subgroup, not included in the two above cases, the degree of $w(x)$ is either 4,6 or 12 .

- Case 4: the subgroup is the whole group $S L(2, C)$, the solutions for $w(\tau)$, if they exist are non-Liouvillian.

In the first three cases a Liouvillian solution might exist, and can be found (if it exists) using Kovacic's algorithm. Kovacic proceeded to translate these cases to a set of necessary but not sufficient conditions that the potential $V(\tau)$ in eq.(5.15) must satisfy for the differential equation to fit in the first three above cases [182]. For each of the cases as ordered above, the corresponding conditions on $V(\tau)$ are:

- Case 1: every pole of $V(\tau)$ has order 1 or has even order. The order of the function $V(\tau)$ at infinity must be either even or greater than 2 .

- Case 2: $V(\tau)$ has at least a single pole of order 2, or of odd-order greater than 2 .

- Case 3: the order of the poles of $V(\tau)$ does not exceed 2, and the order of $V(\tau)$ at infinity is at least 2 .

Here the order of $V(\tau)$ at infinity means the order of infinity as a zero of $V(\tau)$, which is thus equal to the degree of the denominator minus the degree of the numerator.

If none of the above is satisfied, we are dealing with case 4, where the solutions are not invariant under a subgrop of $S L(2, C)$, and the solution (if it exists), is non-Liouvillian.

\subsubsection{Kovacic Analysis}

To apply Kovacic's criteria to the NVE equations for the string, we insert the solution for $z(\tau)$ from eq.(5.11), and expand the solution for the other variables as in eq.(5.13). The result will be a second order differential equation in $\tau$, of the form that Kovacic's algorithm applies to. 


\section{V. (NON)-INTEGRABILE $6 \mathrm{D} \mathcal{N}=(1,0)$ SCFTS}

By rewriting the NVE equations into the form of eq.(5.15) we can then see if the corresponding potential $V(\tau)$ fails to satisfy all three of Kovacic's criteria, in which case we know the solutions of the NVE equation are non-Liouvillian and the string worldsheet on this background is non-integrable.

\section{NVE for $\rho$}

If we now allow for small fluctuations in $\rho(\tau)=0+\epsilon r(\tau)$ and insert the solution for $z_{\text {sol }}(\tau)$ in (5.12) into the equation of motion for $\ddot{r}(\tau)$, we find for the NVE

$$
\begin{aligned}
& \ddot{r}(\tau)+\mathcal{B}_{r}(\tau) \dot{r}(\tau)+\mathcal{A}_{r}(\tau) r(\tau)=0 \\
& \mathcal{B}_{r}(\tau)=\left.\frac{f_{1}^{\prime}(z)}{f_{1}(z)} \frac{E}{4 \pi}\right|_{z_{s o l}}=\left.\frac{E}{8 \pi}\left(\frac{\alpha^{\prime}}{\alpha}-\frac{\alpha^{\prime \prime \prime}}{\alpha^{\prime \prime}}\right)\right|_{z_{s o l}} \\
& \mathcal{A}_{r}(\tau)=\left.\frac{E^{2}}{f_{1}(z)^{2}}\right|_{z_{\text {sol }}}=\left.\frac{-E}{128 \pi^{2}} \frac{\alpha^{\prime \prime}}{\alpha}\right|_{z_{\text {sol }}}
\end{aligned}
$$

When only considering a string that moves along the $z$ and $\rho$-directions, it is now easy to see that if the warp factor $f_{1}(z)$ as defined in eq.(4.7) is equal to a constant, $\mathcal{B}_{r}=0$, and the above differential equation admits a Liouvillian solution of the form $r(\tau)=\exp (i E \tau)$. We will examine this particular case in more detail in the next chapter.

When we allow for a warp factor between the $\mathrm{AdS}_{7}$ and $\mathcal{M}^{3}$ spaces, such that $f_{1}(z)$ is no longer equal to a constant, we can use Kovacic's algorithm [151] to try to determine if the resulting differential equation (5.17) might admit any Liouvillian solutions. This can be done by combining the coefficients $\mathcal{A}(\tau)$ and $\mathcal{B}(\tau)$ of the second order differential equation into the new function $V(\tau)$. For the differential equation (5.17) to possibly admit a Liouvillian solution, the function $V(\tau)$ has to satisfy at least one of the necessary but not sufficient conditions listed in the previous section. In this case of the NVE for $\rho$ in eq.(5.17), the function $V_{r}(\tau)$ is of the form

$$
\begin{aligned}
V_{r} & =\frac{1}{4}\left(2 \mathcal{B}_{r}^{\prime}+\mathcal{B}_{r}^{2}-4 \mathcal{A}_{r}\right) \\
& =\frac{E^{2}}{256 \pi^{2} \alpha^{2}\left(\alpha^{\prime \prime}\right)^{2}}\left(-3\left(\alpha^{\prime}\right)^{2}\left(\alpha^{\prime \prime}\right)^{2}-2 \alpha \alpha^{\prime} \alpha^{\prime \prime} \alpha^{\prime \prime \prime}+\alpha\left(6\left(\alpha^{\prime \prime}\right)^{3}+5 \alpha\left(\alpha^{\prime \prime \prime}\right)^{2}-4 \alpha \alpha^{\prime \prime} \alpha^{\prime \prime \prime \prime}\right)\right)
\end{aligned}
$$

Remember that $\alpha(z)$ is the function that specifies the quiver structure corresponding to the $\mathrm{AdS}_{7}$ geometry, as defined in eq.(4.8). We obtained the NVE by expanding around a solution for $z$ which is of the form $z_{\text {sol }}=\frac{E}{4 \pi} \tau$, so that $V(\alpha(z(\tau)))$ is in the end a function of $\tau$ only.

If we construct a function $\alpha(z)$ from a quiver diagram, $\alpha(z)$ will be a piece-wise continuous polynomial of at most order 3 at every point.

$$
\alpha(z)=a_{3} z^{3}+a_{2} z^{2}+a_{1} z+a_{0}
$$


Inserting this solution for $\alpha(z)$ into the potential in eq. (5.18) will allow us to analyse the pole structure of $V_{r}(\tau)$ corresponding to a general quiver diagram,

$$
V_{r}(\tau)=\frac{3 E^{2}}{2} \frac{3 a_{3}^{4} E^{6} \tau^{6}+24 a_{2} a_{3}^{3} E^{5} \pi \tau^{5}+\ldots}{\left(4 a_{2} \pi+3 a_{3} E \tau\right)^{2}\left(64 a_{0} \pi^{3}+16 a_{1} E \pi^{2} \tau+4 a_{2} E^{2} \pi \tau^{2}+a_{3} E^{3} \tau^{3}\right)^{2}} .
$$

The dots in the numerator are quartic terms in $\tau$, the order of $V(\tau)$ at infinity is thus equal to two. Note that $V_{r}(\tau)$ has one pole of order two at $\tau=r_{0}=-4 a_{2} \pi / 3 a_{3} E$. The other poles come from the cubic polynomial that is the second term in the denominator, one of the real roots of this cubic polynomial can coincide with the earlier pole $\tau=r_{0}$.

Let us first examine the possible roots coming from this cubic polynomial: A cubic polynomial of the form $a \tau^{3}+b \tau^{2}+c \tau+d=0$ has three complex roots, the multiplicity of which can be obtained from the determinant

$$
\begin{aligned}
\Delta & =a^{2} b^{2}-4 b^{3}-4 a^{3} c-27 c^{2}+18 a b c \\
& =-4096\left(-a_{1}^{2} a_{2}^{2}+4 a_{1}^{3} a_{3}-18 a_{0} a_{1} a_{2} a_{3}+a_{0}\left(4 a_{2}^{3}+27 a_{0} a_{3}^{2}\right)\right) E^{6} \pi^{6} .
\end{aligned}
$$

If $\Delta>0$ the polynomial has three distinct real roots, for $\Delta<0$ there is one real root and two complex conjugate roots. When $\Delta=0$ there are roots with multiplicity larger than one, this can be either a single real root of multiplicity 3, or one real root of multiplicity 2 and another additional root. If we list all possible options we arrive at the following table where the orders of the poles of $V_{r}(\tau)$ are listed in both the case when first pole $r_{0}$ does coincide with one of the real poles from the cubic term $\left(r_{0}=r_{1}\right)$, and when this does not happen. We see that in all

\begin{tabular}{|l|l|l|l|}
\cline { 2 - 4 } \multicolumn{1}{c|}{} & cubic poles & $r_{0} \neq r_{1}$ & $r_{0}=r_{1}$ \\
\hline$\Delta>0$ & $\left(\tau-r_{1}\right)\left(\tau-r_{2}\right)\left(\tau-r_{3}\right)$ & $2,2,2,2$ & $4,2,2$ \\
\hline$\Delta=0$ & $\left(z-r_{1}\right)^{3}$ & 2,6 & 8 \\
\hline$\Delta=0$ & $\left(z-r_{1}\right)^{2}\left(z-r_{2}\right)$ & $2,4,2$ & 6,2 \\
\hline$\Delta=0$ & $\left(z-r_{1}\right)\left(z-r_{2}\right)^{2}$ & $2,2,4$ & 4,4 \\
\hline$\Delta<0$ & $\left(\tau-r_{1}\right)\left(\tau-c_{2}\right)\left(\tau-c_{3}\right)$ & $2,2,2,2$ & $4,2,2$ \\
\hline
\end{tabular}

cases all poles will be of even order, and that the resulting $V_{r}(\tau)$ thus might pass the first and second of Kovacic's criteria. These conditions are necessary but not sufficient to guarantee the existence of Liouvillian solutions. Let us next turn to the string soliton moving along the $\varphi$ direction.

\section{NVE for $\varphi$}

We now examine the equation of motion for $\ddot{\varphi}$. If we allow for small fluctuations in $\varphi(\tau)=$ $0+\epsilon f(\tau)$, and we insert the solution for $z(\tau)$ from eq.(5.12) while we now freeze the string and 


\section{V. (NON)-INTEGRABILE $6 \mathrm{D} \mathcal{N}=(1,0)$ SCFTS}

let it not move along the other directions such that $\rho=\dot{\rho}=\ddot{\rho}$ we find for the NVE

$$
\begin{aligned}
& \ddot{f}(\tau)+\mathcal{B}(\tau) \dot{f}(\tau)+\mathcal{A}(\tau) f(\tau)=0 \\
& \mathcal{B}_{f}(\tau)=\left.\frac{f_{1}^{\prime}(z)}{f_{1}(z)} \frac{E}{4 \pi}\right|_{z_{s o l}}=\left.\frac{E}{8 \pi}\left(\frac{\alpha^{\prime}}{\alpha}-\frac{\alpha^{\prime \prime \prime}}{\alpha^{\prime \prime}}\right)\right|_{z_{s o l}} \\
& \mathcal{A}_{f}(\tau)=\lambda^{2}
\end{aligned}
$$

Again, we see that when $f_{1}(z)$ is equal to a constant, the above NVE will reduce to the harmonic oscillator with solution $a(\tau)=\exp (i \lambda \tau)$.

When we allow for a more general warp factor where $f_{1}(z)$ is no longer constant, we can again use Kovacic's algorithm to determine if we above differential equation might still admit Liouvillian solutions. Inserting again for $\alpha(z)$ a general third order polynomial as given in eq.(5.19) gives us for the potential

$$
V_{f}(\tau)=\frac{-36 a_{3}^{4} E^{8} \lambda^{2} \tau^{8}-384 a_{2} a_{3}^{3} E^{7} \pi \lambda^{2} \tau^{7}+\ldots}{4\left(4 a_{2} \pi+3 a_{3} E \tau\right)^{2}\left(64 a_{0} \pi^{3}+16 a_{1} E \pi^{2} \tau+4 a_{2} E^{2} \pi \tau^{2}+a_{3} E^{3} \tau^{3}\right)^{2}}
$$

Notice that though the order of the numerator is different from $V_{r}(\tau)$, the pole structure in the denominator is identical to that in eq.(5.20). Since the numerator does now contains terms $\tau^{8}$, the order of $V(\tau)$ at infinity is zero, as $V_{f}(\tau) \sim \lambda^{2}$ when $\tau \rightarrow \infty$. We thus see immediately that $V_{f}(\tau)$ fails to meet the third of Kovacic's criteria. However, as before, since all the poles are always of even order, the first and second of Kovacic's criteria will always be satisfied.

\section{Conditions to violate the Second Criterion}

As we have seen the NVE's for $\rho$ and $\varphi$ always violate the third of Kovacic's criteria, and always satisfy the first. Satisfying any of the criteria is however not a sufficient condition to guarantee the existence of a Liouvillian solution. By applying Kovacic's algorithm for the corresponding cases one can either find the Liouvillian solution, or show it does not exist. It would be interesting to carry out the algorithm for case 1 explicitly, to see for which combinations of the constants $a_{i}$ it would fail to provide a Liouvillian solution. With this in mind we here present conditions that will guarantee the second of Kovacic's criteria cannot be met, in which case the algorithm for case 1 would be sufficient to either find the Liouvillian solutions or show they do not exist.

Since all the poles for the resulting potentials $V(\tau)$ are always even, the second criteria is only not satisfied in two cases

- When the roots of the cubic polynomial in the denominator are all equal to $\tau=\frac{4 \pi a_{2}}{3 E a_{3}}$ so there is only a single pole of order 8 . This happens when

$$
\begin{aligned}
\left(4 a_{2} \pi+3 a_{3} E \tau\right)^{3} & =64 a_{0} \pi^{3}+16 a_{1} E \pi^{2} \tau+4 a_{2} E^{2} \pi \tau^{2}+a_{3} E^{3} \tau^{3} \\
& =64 \pi^{3} a_{2}^{3}+144 a_{2}^{2} a_{3} \pi^{2} E \tau+108 a_{2} a_{3}^{2} \pi E^{2} \tau^{2}+27 a_{3}^{3} E^{3} \tau^{3}
\end{aligned}
$$


which happens when the functions $a_{i}$ satisfy the conditions

$$
a_{0}=a_{2}^{3}, \quad a_{3}=0
$$

- When only one of the roots of the cubic polynomial in the denominator is equal to $\tau=\frac{4 \pi a_{2}}{3 E a_{3}}$, there are only two poles of order 4 . This means we should be able to factorise the cubic polynomial as

$$
\left(4 a_{2} \pi+3 a_{3} E \tau\right)\left(A \tau^{2}+B \tau+C\right)=64 a_{0} \pi^{3}+16 a_{1} E \pi^{2} \tau+4 a_{2} E^{2} \pi \tau^{2}+a_{3} E^{3} \tau^{3}
$$

This factorisation is only possible when the coefficients $a_{i}$ are related to one another so that

$$
a_{1}=5 a_{0} a_{3}+\frac{4 a_{2}^{2}}{27 a_{3}}
$$

Demanding that $\tau=\frac{4 \pi a_{2}}{3 E a_{3}}$ is not a root of the remaining quadratic polynomial, so there are two poles of order 4 , implies

$$
\left(\frac{27 a_{0} a_{3}^{2}-a_{2}^{2}}{48 \pi^{2} a_{2}^{4}}\right) E^{2} \tau^{2}+\left(\frac{a_{2}^{2}-27 a_{0} a_{3}^{2}}{27 \pi a_{2}^{3} a_{3}}\right) E \tau+\left.\frac{a_{0}}{a_{2}^{2}}\right|_{\tau=\frac{4 \pi a_{2}}{3 E a_{3}}} \neq 0
$$

which we can then translate to another condition on the coefficients $a_{i}$,

$$
a_{3}^{2} \neq \frac{-a_{2}^{2}}{54 a_{0}}
$$

It would be interesting to carry out Kovacic's algorithm using the above conditions where both the second and third of Kovacic's criteria are not satisfied. We could then find conditions on the coefficients $a_{i}$ that guarantee Kovacic's algorithm fails to give an Liouvillian solution on the interval $\left[z_{i}, z_{i+1}\right]$, analoguous to how this is done in [132].

\section{NVE for $\chi$}

If we allow for small fluctuations in $\chi(\tau)=0+\epsilon x(\tau)$ and insert the solution in the equation of motion for $\ddot{\chi}(\tau)$ eq.(5.12), we find for the NVE for $\ddot{x}(\tau)$

$$
\begin{aligned}
\ddot{x}(\tau)+\mathcal{B}_{x}(\tau) \dot{x}(\tau)+\mathcal{A}_{x}(\tau) x(\tau)=0 & =\left.\frac{E f_{3}^{\prime}(z)}{4 \pi f_{3}(z)}\right|_{z_{s o l}}=\left.\frac{E}{8 \pi}\left(3 \frac{\alpha^{\prime}}{\alpha}+\frac{\left(\alpha^{2}+2 \alpha \alpha^{\prime \prime}\right) \alpha^{\prime \prime \prime}}{\left(\alpha^{2}-2 \alpha \alpha^{\prime \prime}\right) \alpha^{\prime \prime}}\right)\right|_{z_{s o l}} \\
\mathcal{B}_{x}(\tau) & =\left.\left(\kappa^{2}-\kappa \frac{E f_{4}^{\prime}(z)}{4 \pi f_{3}(z)}\right)\right|_{z_{s o l}} \\
& =\left.\left(\kappa^{2}-\frac{E \kappa}{4 \pi} \frac{1}{\sqrt{-2 \alpha \alpha^{\prime \prime}}} \frac{6 \alpha \alpha^{\prime \prime 2}-2 \alpha \alpha^{\prime} \alpha^{\prime \prime \prime}-3 \alpha^{\prime 2} \alpha^{\prime \prime}}{2 \alpha \alpha^{\prime \prime}-\alpha^{\prime 2}}\right)\right|_{z_{s o l}}
\end{aligned}
$$




\section{V. (NON)-INTEGRABILE $6 \mathrm{D} \mathcal{N}=(1,0)$ SCFTS}

We now only consider fluctuations of the string along the $z$ and $\chi$-direction, which is the same situation that was considered in [136]. Because the function $V_{x}(\tau)$ now involves $f_{3}(z)$ and $f_{4}(z)$ and their derivatives, it is far less obvious to see from this result that the string soliton will directly fail to be integrable when $f_{1}(z)$ is not equal to a constant (as we obtained from the NVE's for $\rho$ and $\varphi$ ). If we would again insert a general function $\alpha(z)$ of the form given in eq.(5.19), the resulting $V_{x}(\tau)$ will be a complicated sum of large fractions. We will omit the result here.

It is quite difficult to extract general expressions for these cases, as could be done more nicely for the NVE's for $\rho$ and $\varphi$, where we easily saw that when $f_{1}(z)$ is constant, the corresponding string embedding is integrable.

\subsubsection{Results for Various $\mathrm{AdS}_{7}$ Backgrounds}

We will now apply the results of the previous section to various specific $\mathrm{AdS}_{7}$ backgrounds:

\section{Linear Quiver}

We will continue by illustrating Kovacic's method for a linear rank function with $\alpha(z)=$ $-81 \pi^{2} N\left(\frac{1}{6} z^{3}-\frac{5}{2} z\right)$ for $z \in\left[0, z_{n}\right]$ at the beginning of the $z$-interval. We thus have $a_{0}=$ $0, a_{1}=\frac{5}{2}, a_{2}=0$, and $a_{3}=\frac{1}{6}$. In eq.(5.23) and (5.18) the denominator then simplifies to

$$
4\left(3 a_{3} E \tau\right)^{2}\left(16 a_{1} E \pi^{2} \tau+a_{3} E^{3} \tau^{3}\right)^{2}
$$

Giving us one pole of order 4, and two poles of order 2 for the potential corresponding to the NVE's for $\rho$ and $\varphi$. The second of Kovacic's criteria will therefore still be satisfied by both potentials, and a Liouvillian solution might exist. The potential $V_{f}(\tau)$ also satisfies the second criterion.

The coefficients in the NVE for $\chi$ in this case are

$$
\begin{aligned}
& \mathcal{A}=1-\sqrt{3} \frac{\left(z^{4}+20 a_{1} z^{2}-60 a_{1}^{2}\right)}{\sqrt{-6 a_{1}-z^{2}}\left(z^{4}+12 a_{1} z^{2}-12 a_{1}^{2}\right)}, \\
& \mathcal{B}=\frac{2}{z}+\frac{3 z}{\left(6 a_{1}+z^{2}\right)}-4 \frac{6 a_{1} z+z^{3}}{\left(z^{4}+12 a_{1} z^{2}-12 a_{1}^{2}\right)} .
\end{aligned}
$$

Note that when we rescale $z \rightarrow n z$ and take the limit $n \rightarrow \infty$ the above equation becomes a simple oscillator. This is reminiscent of the integrability of strings on the linear quiver back-

grounds that show up in the $\frac{1}{2}$ BPS solutions as non-Abelian T-dualities of more symmetric backgrounds (a well known example is the Sfetsos-Thompson background). 
Next we will perform a more detailed analysis for finite $n$. To avoid cluttering the expressions, we have chosen the coefficients $E=4 \pi$ (such that $z=\tau$ ) and $\nu=1$. The coefficients of this NVE are not rational functions. To amend this, we change from $z$ to a new variable $v$,

$$
z=\sqrt{-6 a_{1}-v^{2}}
$$

denoting $x^{\prime}=\frac{d x}{d v}$, the NVE reads

$$
x^{\prime \prime}(v)+\mathcal{C} x^{\prime}(v)+\mathcal{D} x(v)=0, \quad \mathcal{C}=\frac{\left(\mathcal{B}(v)+\frac{d}{d v} \frac{d v}{d z}\right)}{\frac{d v}{d z}}, \quad \mathcal{D}=\frac{\mathcal{A}(v)}{\left(\frac{d v}{d z}\right)^{2}} .
$$

According to what was explained around eq.(5.15), we now need to analyse the principal part of the potential $4 V=2 \frac{d \mathcal{C}}{d v}+\mathcal{C}^{2}-4 \mathcal{D}$. We find,

$$
\begin{aligned}
& \mathcal{C}=\frac{v^{6}-12 a_{1} v^{4}-240 a_{1}^{2} v^{2}-576 a_{1}^{3}}{v\left(v^{2}+6 a_{1}\right)\left(v^{4}-48 a_{1}^{2}\right)}, \\
& \mathcal{D}=-1+\frac{6 a_{1}+5 \sqrt{3} v}{v^{2}+6 a_{1}}-\frac{4 \sqrt{3} v\left(v^{2}-4 a_{1}\right)}{v^{4}-48 a_{1}^{2}}, \\
& 4 V=4+\frac{\gamma_{0}}{v^{2}}+\frac{\gamma_{1}}{\left(v^{2}+6 a_{1}\right)^{2}}+\frac{\gamma_{2}+\gamma_{3} v}{\left(v^{2}+6 a_{1}\right)}+\frac{\gamma_{4} v^{2}}{\left(v^{4}-48 a_{1}^{2}\right)^{2}}+\frac{\gamma_{5}+\gamma_{6} v+\gamma_{7} v^{2}+\gamma_{8} v^{3}}{\left(v^{4}-48 a_{1}^{2}\right)} .
\end{aligned}
$$

The coefficients $\gamma_{i}$ are numerical constants, not very relevant for our analysis below. Notice the leading orders of the poles are all even and the order of the potential (leading power of the degrees of the denominator minus numerator) is zero. Hence, $V(x)$ does satisfy the conditions

for the first and second of Kovacic's criteria, and a Liouvillian solution might exist. Further numerical studies show this is not the case for linear quivers that end with a flavour group.

\section{Massless Solution}

We now apply Kovacic's criteria to the NVE equations for the massless solution in section 5.5.3. When the Romans mass $F_{0} \sim \alpha^{\prime \prime \prime}=0$, the corresponding function $\alpha(z)$ is

$$
\alpha(z)=-81 \pi^{2} k\left(\frac{1}{2} z^{2}-\frac{2 R_{0}^{2}}{81 \pi^{2} k^{2}}\right)=R^{2}-\mu z^{2} .
$$

For this solution $a_{0}=R^{2}, a_{1}=0, a_{2}=-\mu$, and $a_{3}=0$. In the potentials $V_{x}$ and $V_{f}$, eq.(5.23) and (5.18), the denominator then simplifies to

$$
4\left(4 a_{2} \pi\right)^{2}\left(64 a_{0} \pi^{3}+4 a_{2} E^{2} \pi \tau^{2}\right)^{2}
$$

Giving us three poles of order 2. The second of Kovacic's criteria will therefore still be satisfied by both potentials, and a Liouvillian solution might exist. 
We find that the NVE equation for $\chi$ in eq.(5.28) reads in this case,

$$
\ddot{x}(\tau)-\frac{243 E^{2} k^{2} \pi^{2} \tau}{16 \pi^{2}\left(4 R_{0}^{2}-81 k^{2} \pi^{2}\left(\frac{E \tau}{4 \pi}\right)^{2}\right)} \dot{x}(\tau)+\nu\left[\nu+\frac{27 k E \pi}{4 \pi \sqrt{4 R_{0}^{2}-81 k^{2} \pi^{2}\left(\frac{E \tau}{4 \pi}\right)^{2}}}\right] x(\tau)=0 .
$$

This equation is hard to solve exactly, let us distinguish two cases: We observe that for very large values of the parameter $R_{0}$ (or for very short times), the eq.(5.38) reduces to an oscillator equation.

Let us now perform a more refined analysis for finite $R_{0}$ following Kovacic's algorithm. The first step is to write the NVE as a second order differential equation with rational coefficients. We choose $64 R_{0}^{2}=81 k^{2} E^{2}=1$ to ease the algebra (not loosing generality). The NVE equation reads,

$$
\ddot{x}-\frac{3 \tau}{1-\tau^{2}} \dot{x}+\left(1+\frac{3}{\sqrt{1-\tau^{2}}}\right) x=0 .
$$

We change variables to $\tau=\sqrt{1-v^{2}}$. The NVE differential equation in this new variable reads,

$$
x^{\prime \prime}(v)+\mathcal{C}(v) x^{\prime}(v)+\mathcal{D}(v) x(v)=0, \quad \mathcal{C}=\frac{1}{\frac{d v}{d \tau}}\left(\mathcal{B}(v)+\frac{d}{d v}\left(\frac{d v}{d \tau}\right)\right), \quad \mathcal{D}=\frac{\mathcal{A}(v)}{\left(\frac{d v}{d \tau}\right)^{2}} .
$$

Where, in this particular case we have

$$
\begin{array}{ll}
v=\sqrt{1-\tau^{2}}, & \frac{d v}{d \tau}=-\frac{\sqrt{1-v^{2}}}{v}, \quad \frac{d}{d v}\left(\frac{d v}{d \tau}\right)=\frac{1}{v^{2} \sqrt{1-v^{2}}}, \\
\mathcal{C}=\frac{3 v^{2}-4}{v-v^{3}}, & \mathcal{D}=\frac{v^{2}+3 v}{1-v^{2}} .
\end{array}
$$

Following the analysis detailed in section 5.3.1, we construct a function $4 V(v)=4 \mathcal{D}-\mathcal{C}^{2}-2 \mathcal{C}^{\prime}$,

$$
4 V=-4+\frac{3}{4(v-1)^{2}}-\frac{17}{4(v-1)}-\frac{24}{v^{2}}+\frac{3}{4(v+1)^{2}}-\frac{31}{4(v+1)} .
$$

The existence of poles of order two and the fact that the function $V$ is of order zero at infinity, implies the first two of Kovacic's criterea are satisfied. Therefore, the equation might admit a Liouvillian solution. Further inspection numerical however shows this is not the case.

\section{Vanishing Warp Factor}

Let us conclude this section by examining the NVE for $\chi$ in the case the warp factor $f_{1}(z)$ is constant. In this case $\alpha(z)=A \sin \omega z$, and the geometry is given in eq.(6.3-6.5), and $f_{3}(z)$ and $f_{4}(z)$ are given by

$$
f_{3}(z)=\frac{\sqrt{2} \pi}{\omega}\left(\frac{\sin ^{2} \omega z}{1+\sin ^{2} \omega z}\right), \quad f_{4}(z)=\pi\left(-z+\frac{\sin \omega z \cos \omega z}{\omega\left(1+\sin ^{2} \omega z\right)}\right)
$$


The NVE for $\chi(5.28)$ is then given by

$$
\ddot{x}(\tau)+\left(\frac{4 \pi \cot \tau}{3 \pi-\cos 2 \tau}\right) \dot{x}(\tau)+\left(\kappa^{2}-\kappa \frac{(-11+\cos 2 \tau)}{\sqrt{2}(-3+\cos 2 \tau)}\right) x(\tau)=0
$$

where we set $E=4 \pi / \omega$. To obtain a differential equation with rational coefficients we make the change of coordinates $\tau=\arcsin t$, and obtain

$$
\ddot{x}(\tau)+\left(\frac{2\left(t^{4}+2 t^{2}-1\right)}{t\left(t^{4}-1\right)}\right) \dot{x}(\tau)+\frac{-\kappa}{2\left(t^{4}-1\right)}\left(\sqrt{2}\left(t^{2}+5\right)+2\left(t^{2}+1\right) \kappa\right) x(\tau)=0
$$

giving us for the potential

$$
\begin{aligned}
V_{x}(t) & =\frac{1}{4}\left(2 \mathcal{B}_{x}^{\prime}+\mathcal{B}_{x}^{2}-4 \mathcal{A}_{x}\right) \\
& =\frac{1}{2\left(t^{4}-1\right)}\left(2\left(t^{2}-1\right)\left(t^{2}+1\right)^{2} \kappa^{2}+\sqrt{2}\left(t^{6}+5 t^{4}-t^{2}-5\right)-4\left(t^{4}-2 t^{2}+3\right)\right)
\end{aligned}
$$

Note the potential has four poles of order 1 , and at infinity is of order 4, and thus satisfies the first of Kovacic's criteria. A Liouvillian solution might indeed exist. We will show in chapter 6 this is indeed the case, and the entire (bosonic) sigma model of the string is integrable on the target space for $\alpha(z)=A \sin \omega z$.

\subsection{Numerical Indicators of Chaos}

In this section, we introduce the numerical tools that we will use in the next section to show the equations of motion for the strings (5.7) on various backgrounds are chaotic, and non-integrable. These numerical tools are power spectra, Poincaré sections, and Lyapunov exponents [161].

\subsubsection{Power Spectra}

The time evolution of a chaotic system will look disorganised and non-periodic. By simply taking the Fourier transform of the time evolution of a system, one can make a first crude distinction whether the time evolutions is periodic, quasi-periodic or chaotic. When a signal is perfectly periodic with a frequency $\omega$, its Fourier spectrum will show a vertical line at the characteristic frequency of the system. For a quasi-periodic evolution these peaks broaden, when the dynamics is uncontrollably chaotic the Fourier spectrum becomes flat.

\subsubsection{Poincaré Sections and KAM Tori}

An $N$-dimensional integrable system possesses $N$ independent integrals of motion that are in involution, namely, the Poisson bracket of any two of these conserved quantities vanishes. As 


\section{V. (NON)-INTEGRABILE $6 \mathrm{D} \mathcal{N}=(1,0)$ SCFTS}

a consequence of this, the corresponding phase space trajectories are confined to the surface of an $N$-dimensional KAM torus [161]. When we change our variables to action-angle variables $\left(q_{i}, p_{i}\right) \rightarrow\left(\phi_{i}, J_{i}\right)$, such that our Hamiltonian only depends on $J_{i}$, the corresponding trajectories on this KAM torus are completely specified in terms of $N$ frequencies $\left(\omega_{i}\right)$ that specify the velocities along the different angles on this torus. When there is no set of integers $n^{i}$ such that $\omega_{i} n^{i}=0$, these trajectories are said to be quasi-periodic, they do not close on themselves but fill the surface of a KAM torus.

As a consequence of this, we can see whether a system is integrable or not, by taking crosssections of its phase-space trajectories. When we plot for example $\left(\phi_{1}, J_{1}\right)$ every time $\phi_{2}=0$ we will see a large number of foliated circular KAM curves associated with the 2-dimensional cross-sections of these foliated KAM tori. Such a cross-section is known as a Poincare section [161]. The KAM theorem tells us how these KAM curves will change when we perturb an integrable Hamiltonian with a small deformation $\epsilon H^{\prime}$, where $\epsilon \ll 1$. The resonant tori - for which these trajectories close on themselves, will be destroyed by this perturbation. However, a large number of these non-resonant KAM tori will survive. As we continue to increase the strength of this perturbation, more and more of these tori are destroyed until the motion becomes seemingly random and we loose all of the KAM curves in our Poincaré section.

As an example we will briefly illustrate this for the Henon-Heiles system, which is obtained from the Hamiltonian

$$
\mathcal{H}=\frac{1}{2}\left(x^{2}+y^{2}+2 x^{2} y-\frac{2}{3} y^{3}\right)+\frac{1}{2}\left(p_{x}^{2}+p_{y}^{2}\right) .
$$

This system looks like two coupled oscillators $x$ and $y$, when both $x, y$ and their conjugate momenta $\left(p_{x}\right.$ and $\left.p_{y}\right)$ are small. In addition there is a coupling term $2 x^{2} y$, and an interaction term for $y$ of the form $-\frac{2}{3} y^{3}$, that will become increasingly more important as we explore the system at larger energies.

In figure 5.1 we plot the phase space trajectories for $\left(x(t), p_{x}(t)\right)$ in red, and $\left(y(t), p_{y}(t)\right)$ in blue. We start with initial conditions $x^{(0)}=y^{(0)}=p_{x}^{(0)}=p_{y}^{(0)}=0.01$ and slowly increase the value of $x^{(0)}$ in order to explore larger energies. We see that for larger energies the coupling between the two oscillators becomes increasingly more relevant, causing the dynamics to become non-integrable. This is reflected in the Poincaré sections, where all orbits for the low energy system are located on KAM tori, but this structure breaks apart as we go to larger energies.

When the energies are small, the orbits are periodic and the system is integrable. As we increase the energies, the orbits first become quasi-periodic, and we see in the Poincaré sections that almost all orbits are still located on the KAM tori in phase space. These two-dimensional Poincaré sections are cross sections of the orbits in phase space. Since the orbits are located on KAM tori, the two-dimensional cross sections of the orbits look like circles. To draw the Poincaré sections, we consider a trajectory with fixed energy. Every time one of the phase space variables (here $x$ ) reaches a particular value (here $x=0$ ), we keep track of the values of 

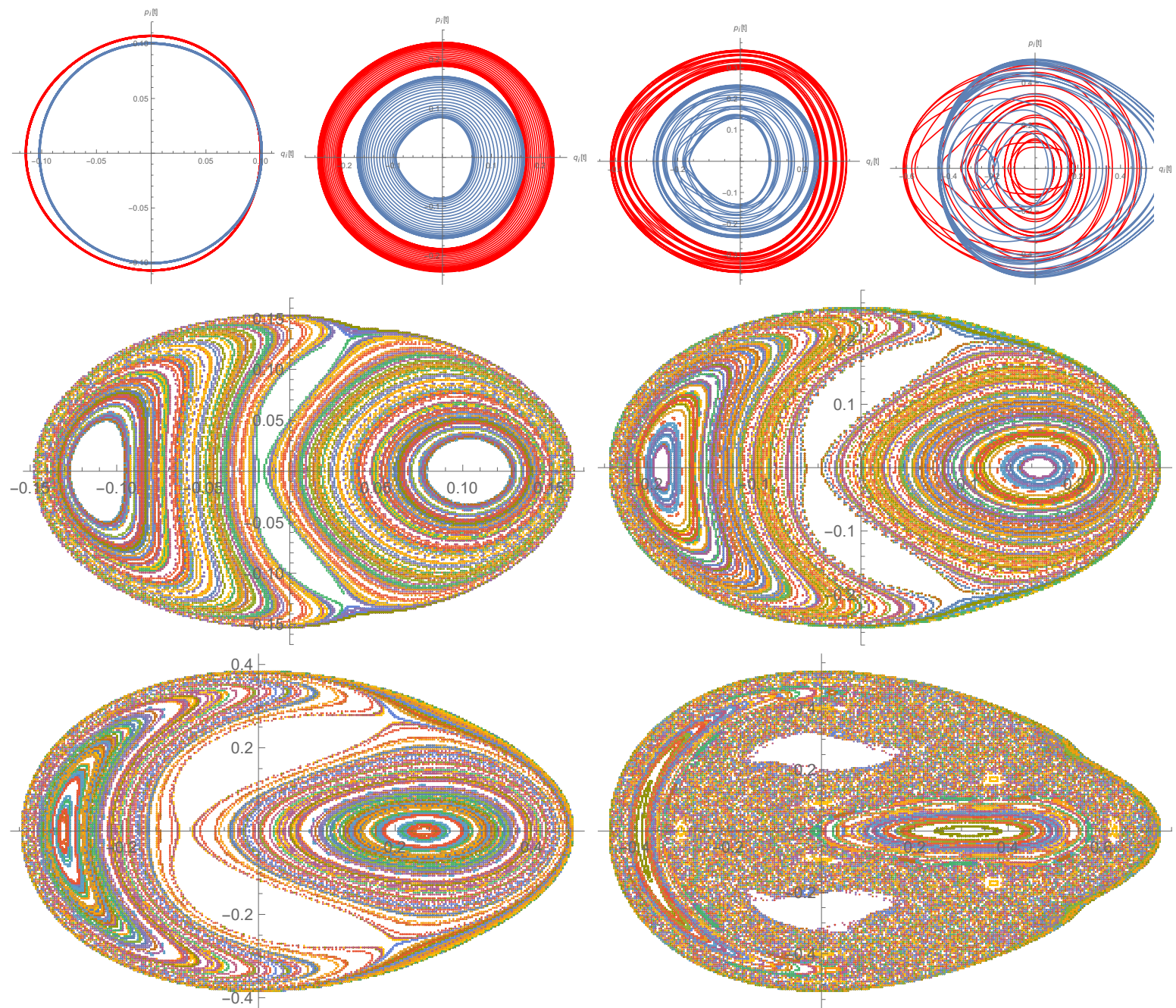

Figure 5.1: (top) trajectories in phase space for the Henon-Heiles system with initial condtitions $x_{0}=y_{0}$ respectively equal to $\frac{1}{10}, \frac{1}{6}, \frac{1}{4}, \frac{1}{3}$. (bottom) Poincaré section of the $\left(y, p_{y}\right)$-plane everytime $x(t)=0$ for 150 different trajectories with energies equal to those of the initial conditions in the top figures.

the other phase-space variables (here $y$ and $p_{y}$ ) and add the point in phase space to our figure. If the trajectory in phase space is indeed located on a KAM torus we expect all the points $\left(y, p_{y}\right)$ (when $x(t)=0$ ) to form a circle that is the cross section of this torus.

As we increase the energies even further we see the trajectories become chaotic. We see this onset of chaos reflected in the large number of KAM tori in phase space that are now destroyed 


\section{V. (NON)-INTEGRABILE $6 \mathrm{D} \mathcal{N}=(1,0)$ SCFTS}

(there can still be some KAM tori left, corresponding to particular orbits that are still periodic, but these diminish in number as we keep increasing the energy).

\subsubsection{Lyapunov Exponents}

The evolution of a dynamical system is given by a set of deterministic differential equations that allows us to calculate the state of a system at a time $t$, knowing an earlier state of the system at some initial time $t_{0}$. A dynamical system is said to be 'chaotic' when it is exponentially sensitive to its initial conditions, making it practically impossible to accurately predict the long term dynamical behaviour, as it would require measuring the initial condition of the system with infinite precision. When we have two adjacent initial conditions $x_{1}\left(t_{0}\right)$ and $x_{2}\left(t_{0}\right)=x_{1}\left(t_{0}\right)+\epsilon$, we say the system exhibits chaotic dynamics when

$$
\left|x_{1}(t)-x_{2}(t)\right| \sim e^{\lambda_{x} t}
$$

provided that the trajectory of our system in phase-space remains bounded. This boundedness of the trajectories is to rule out the trivial case where the trajectories move exponentially to infinity and only diverge exponentially because they are moving apart [161].

Here $\lambda_{x}$ is known as the Lyapunov exponent corresponding to the phase-space variable $x$, and forms a measure of the sensitivity of the system to the initial conditions for this variable. When we have multiple phase space variables (like $x, y, p_{x}$ and $p_{y}$ for the Henon-Heiles system) there is a corresponding Lyapunov exponent for each of them. Since the equations of motion for our string solitons are a Hamiltonian system, the initial volume in phase space will be conserved (as a consequence of Liouville's theorem). This implies the sum of the different Lyapunov exponents will equal zero. The largest Lyapunov exponent (LLE) is then typically used as an indicator to tell us how chaotic the dynamics of the system is.

Note the trajectories of string embedding on a geometry with an 'internal space' will automatically be bounded if the internal space is compact and bounded (in our case the $z$-coordinate is bounded, and the internal space topologically looks like a three-sphere). In this case, the Lyapunov exponent (that measures the exponential divergence of initial conditions) provides a good observable to determine whether the dynamics of this classical string embedding is chaotic or not.

A numerical algorithm to calculate Lyapunov exponents has been included in Appendix 5.6 .

\subsection{Analysis for Various $\mathrm{AdS}_{7}$ Backgrounds}

In this section we will investigate various of the $\mathrm{AdS}_{7}$ geometries for signs of chaos using the tools outlined in the previous section. We numerically solve their equations of motion. Using power spectra, Poincaré sections, and Lyapunov exponents, we list on which of these geometries 
the string embeddings are indicative of chaotic dynamics. The conclusions of this section are as follows:

- In section 5.5.1 we outline the evidence of non-integrable dyanamics that we find for all (finite) quivers with flavour branes, and present Poincaré sections and Lyapunov exponents for an example.

- In section 5.5.2 we discuss linear quivers. For an infinite linear quiver we with rank function $R(z) \sim z$. We find no evidence of non-integrable behaviour, and suggest this solution might be integrable. When we consider a finite linear quiver - by adding a flavour group to the end - we again find clear evidence of non-integrable behaviour for this background and present Poincaré sections and Lyapunov exponents.

- In section 5.5.3 we discuss two limit of the massless solution where $\alpha(z)=R^{2}-\mu z$. In the limit $R \rightarrow \infty$ we find no evidence of non-integrable behaviour, and suggest this case might be integrable. When we consider finite values for $R$ the geometry has singular points at the end of the $z$-interval that now cause the string dynamics to become nonintegrable. In the later part of this section we discuss the uplift of the massless solution to 11-dimensions, where the singular points are removed. We show the string embedding moving along $z$ and $\chi$, wrapping the $\xi$ direction, turns into a toroidal M2-brane that also wraps the eleventh direction. These toroidal M2-brane configurations then turn out to be integrable.

- At last, in section 5.5.4 we consider a somewhat unusual solution, where $\alpha(z)=A \sin \omega z$. Since $\alpha(z)$ is not a piece-wise continuous third order polynomial it is not immediately clear what the corresponding rank function for a quiver would be (we will discuss this in a detail in the next chapter). We directly see from the numerical analysis that this particular function $\alpha(z)$ corresponds to an integrable background!

To simplify our analysis we will only focus on the equations of motion (5.7) for string embeddings that sit at the center of the $\mathrm{AdS}_{7}$ spacetime and move on and wrap the internal space; wrapping the $\xi$ direction and moving along $z$ and $\chi$. Note that the 'energy' of the classical string soliton - the integration constant $E$, that has to be tuned to satisfy the Virasoro constraint (5.8) - is given by

$$
E^{2}=f_{1}(z)^{2}\left(\dot{\rho}^{2}+\sinh ^{2} \rho\left(\dot{\varphi}^{2}+\mu^{2} \sin ^{2} \varphi\right)\right)+f_{2}(z) \dot{z}^{2}+f_{3}(z)\left(\dot{\chi}^{2}+\kappa^{2} \sin ^{2} \chi\right) .
$$

This energy minimises for the point $\rho=\varphi=\chi=0$. Here we will numerically study the dynamics of the string when we increase $\chi$ away from the stable point $\chi=0$ and increase its energy. Note the coordinates $z, \chi$ are bounded, as are the respective momenta along the $p_{z}$ and $p_{\chi}$-directions, due to the conserved Hamiltonian that follows from eq.(5.8). 


\section{V. (NON)-INTEGRABILE $6 \mathrm{D} \mathcal{N}=(1,0)$ SCFTS}

Since $\ddot{\chi}=\dot{\chi}=\chi=0$ (or $\chi=\pi$ ) is a solution to the equations of motion, low energy strings will stay fixed at the north or the south pole of the 2-sphere. For this solution for $\chi(\tau)$, the remaining equation for the motion along $z$ reduces to

$$
2 f_{2}(z) \ddot{z}+\dot{z}^{2} f_{2}^{\prime}(z)+\frac{f_{1}^{\prime}(z)}{f_{1}(z)^{2}} E^{2}=0 .
$$

And has the solution $z_{\text {sol }}(\tau)=\frac{E}{4 \pi} \tau$. As we consider strings with increasing energies $E$, they can move further away from the poles of the two-sphere and will osculate around it, while their dynamics in both $\chi$ and $z$ becomes increasingly complex. This behaviour is generic for all of the $\mathrm{AdS}_{7}$ solutions.

\subsubsection{Analysis for Generic Quivers}

We will first demonstrate our methods for the background in Example 3 ( in eq.(4.28)) of the previous chapter.

In Figure 5.2a-5.2c we see that for very low energies, the string stays at the poles of the two-sphere, it moves along the $z$-direction until it hits the end of the $z$-domain. Then, it turns almost instantaneously from the north pole to the south pole, and stays at its new position as it moves back along the $z$-direction (see, Figure $5.2 \mathrm{~b}$-). We see this periodic behaviour clearly reflected in the corresponding Fourier spectrum in see Figure 5.4a. In fact, we clearly see a fundamental frequency of 0.02 (together with higher harmonics that form the jigsaw and box shaped waveforms) corresponding to the oscillations of the string along the $z$-direction with a period of roughly 55t. The finite width of these peaks is due to numerical inaccuracies.

As we increase the energy, the string moves further away from the poles and explores more of the two-sphere. When we consider an initial $\chi(0)=0.1$ - corresponding to $E \approx 6.75$ - we

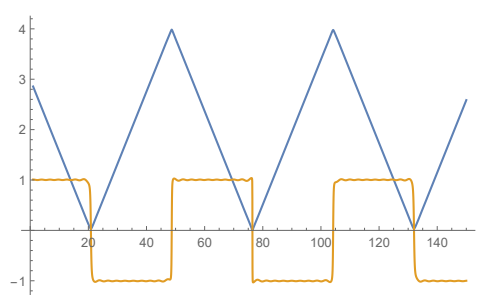

(a)

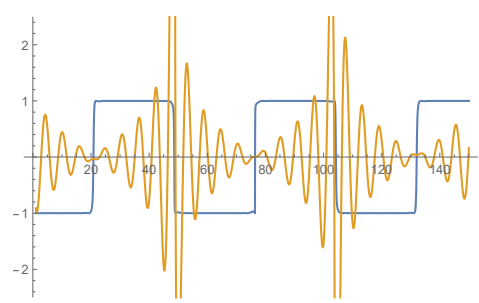

(b)

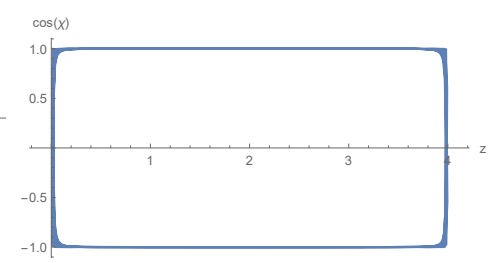

(c)

Figure 5.2: Universal behaviour of strings at very low energies. Numerical evolution with initial conditions $\chi^{(0)}=0.001, p_{\chi}^{(0)}=0, z^{(0)}=2$ and $p_{z}^{(0)}=1$, corresponding $E \approx 3.83$. (a) $z(t)$ and $p_{z}(t)$ in blue and yellow respectively. (b) $\cos \chi(t)$ and $p_{\chi}(t)$ in blue and yellow respectively. (c) Parametric plot of $(z(t), \cos \chi(t))$ with $t_{\max }=400$. 


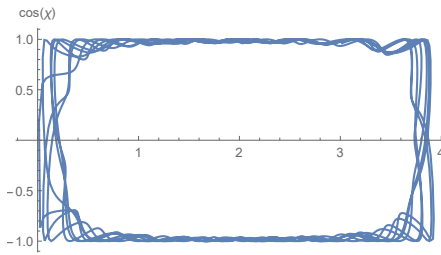

(a)

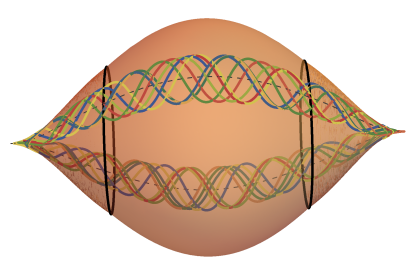

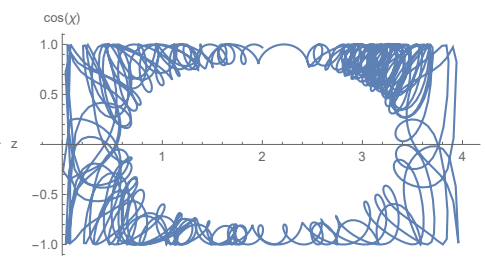

(b)

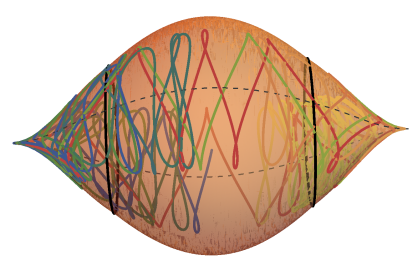

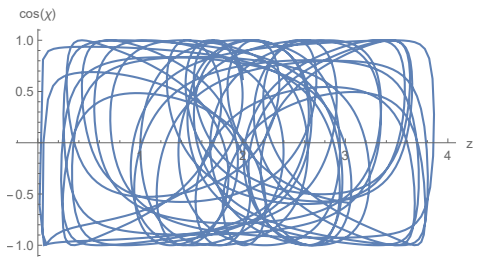

(c)

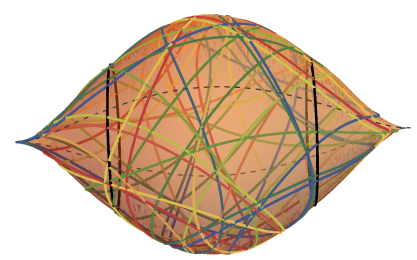

Figure 5.3: Trajectories in the $(z, \chi)$-plane on the background in Example 3 (in eq.(4.28)). We run the evolution till $t=t_{\max }$ and only change the initial $\chi^{(0)}$. (top row) parametric plots of the trajectories in the $(z, \cos \chi)$-plane, with from left to right $\chi^{(0)}=0.10, \chi^{(0)}=0.25$, and $\chi^{(0)}=0.9$ (respectively $E \approx 6.75, E \approx 14.31, E \approx 43.82$ and $t_{\max }=400, t_{\max }=400$, and $\left.t_{\max }=100\right)$. (bottom row) $3 \mathrm{D}$ representation of the same trajectories. The orange surface representing the the two-sphere fibered with warp-factor $f_{3}(z)$ along the $z$-direction. The dashed line indicates the poles of the two-sphere, $\chi=0, \pi$. Points on opposite sides of the dashed line should be identified as $\chi \in[0, \pi]$. The position of the D8-branes is indicated by black lines.

see that the square shaped trajectory that the string traces out in the $(z, \chi)$-plane gets more complicated. As we increase the energy further and allow the string to move further from the poles the trajectories start to look increasingly more disordered.

This is reflected by the corresponding power spectrum, loosing its higher harmonics as shown in Figure $6.2 \mathrm{~b}$. Increasing $\chi(0)$ even further, we see that a broad band of noise around a frequency 0.35 starts to overpower the spectrum, see Figures 6.2b-5.4c. At even higher values of $\chi(0)$, we even loose the initial peak at frequency 0.02. The spectrum becomes primarily dominated by noise.

As we will see throughout this section, this chaotic behaviour at higher energies comes to characterise all of the $\mathrm{AdS}_{7}$ backgrounds we investigate.

In order to generate Poincaré sections we choose a set of different initial conditions, all having 


\section{V. (NON)-INTEGRABILE $6 \mathrm{D} \mathcal{N}=(1,0)$ SCFTS}

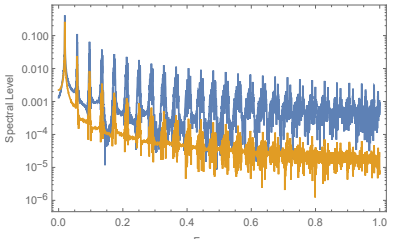

(a)

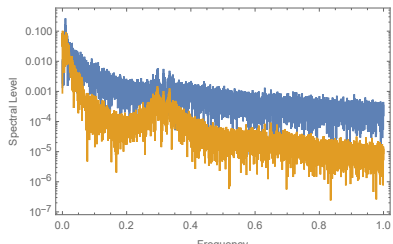

(b)

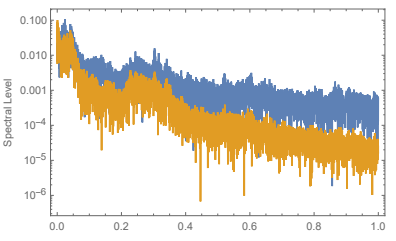

(c)

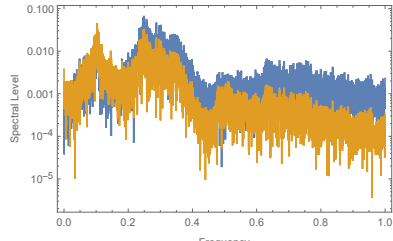

(d)

Figure 5.4: Power spectra corresponding to the trajectories in figures 5.2c-5.3c. Here the spectra for both $z(t)$ and $\chi(t)$ are shown in yellow and blue respectively. To calculate these spectra we ran a numerical evolution up to $t=5000$ (roughly 100 oscillations along the $z$-direction), with a resolution of 10 data-points per time-unit. (a) $\chi_{0}=0.01$, (b) $\chi_{0}=0.1$, (c) $\chi_{0}=0.25$, (d) $\chi_{0}=0.9$.

the same energy $E$. We do this by setting, $z(0)=2, p_{\chi}(0)=0$, and varying $p_{z}(0) \in[0,10]$ and $\chi(0)$ in such a way that the Virasoro constraint in eq. (5.8) is always satisfied for a given energy. We then run the numerical evolution for these initial points, and plot the points $\left(z, p_{z}\right)$ every time $\chi(t)=0$.

If the string motion were integrable, the corresponding trajectories would have been constrained to a 2-dimensional torus in phase-space. The (Poincaré) cross-sections of the phasespace would then show the different resonant tori as embedded lines. The absence of such embedded KAM curves-in Figures 5.5 and 5.9- indicates we are dealing with a non-integrable system.

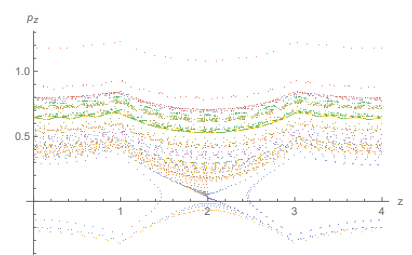

(a) $E=3$

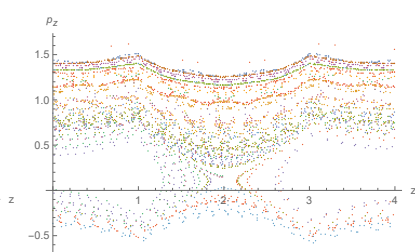

(b) $E=5$

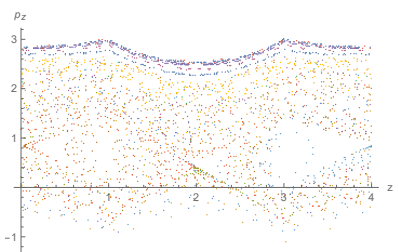

(c) $E=10$

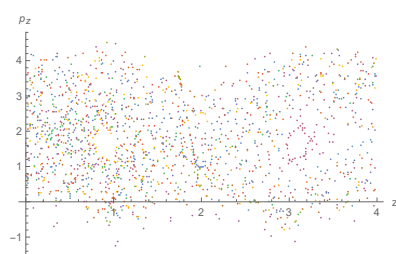

(d) $E=15$

Figure 5.5: Poincaré sections for the $\left(z, p_{z}\right)$-plane at $\chi(t)=0$ at different energies, for the quiver in Example 3.

Note that at low energies we do see the presence of horizontal lines in the Poincaré section. This is because the low energy trajectories of the string fluctuate regularly around the poles of the two-sphere. We are however interested in the possible integrability of all classical string configurations with all possible energies. At higher energies, when the motion of the string 


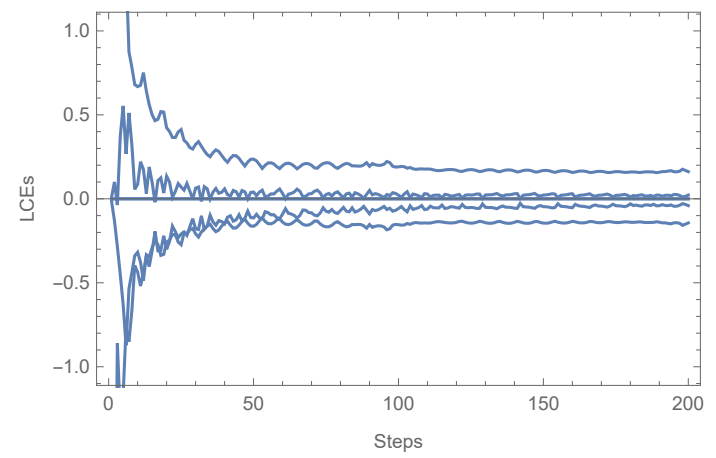

Figure 5.6: Lyapunov spectrum for the quiver in Example 3, for initial conditions $t=0, z=$ $0.09, \chi=0.09, p_{t}=9, p_{z}=0.821405, p_{\chi}=0.22$. Integration: $\tau=0.5, K=200, T=0.02$

becomes increasingly disorganised, these horizontal lines start to disappear and the Poincaré section looses all of its structure, indicating the system is non-integrable.

At last we obtain the Lyapunov exponents in figure 5.6. We find a non-zero Lyapunov exponent, as we would expect for a chaotic system.

We find the kind of chaotic behaviour ilustrated here to be universal for all (finite) quivers with flavour branes. ${ }^{2}$

One way of thinking about this is that since the D8 flavour branes introduce 'kinks' in the rank function, and therefore introduce 'kinks', or discontinuities in the derivative of $\alpha(z)$. It seems that for a general quiver solution, these kinks distort the trajectories of the string in ways that cause the non-integrable behaviour. As we will come to see the only 'normal' quiver backgrounds on which the string seems to integrable are an infinite linear quiver, and an infinitely long massless solution. Both of these geometries are characterised by the absence of flavour branes (or singular points).

\subsubsection{Analysis for a Linear Quiver}

Let us next consider the linear rank function $R(z)=z$, with corresponding

$$
\alpha(z)=-81 \pi^{2} N\left(\frac{1}{6} z^{3}-\frac{5}{2} z\right)
$$

Note that $\alpha(z)$ starts out like this for $z \in[0,1]$ for every rank function, as the first part of the rank function will always be linear.

\footnotetext{
${ }^{2}$ We will discuss infinite quivers that give rise to continuous rank functions in the next section
} 


\section{V. (NON)-INTEGRABILE $6 \mathrm{D} \mathcal{N}=(1,0)$ SCFTS}

\section{Infinite Linear Quiver}

From the Kovacic analysis we saw the NVE for $\chi$ has a Liouvillian solution for an infinite linear rank function. To obtain the corresponding warp factors $f_{i}(z)$ we expand $\alpha(z)$ around $z \rightarrow 0$

$$
f_{1}(z)=8 \pi \sqrt{5}, \quad f_{2}(z)=\frac{2 \pi}{\sqrt{5}}, \quad f_{3}(z)=\frac{2 \pi z^{2}}{\sqrt{5}}, \quad f_{4}(z)=-\frac{2 \pi z^{3}}{3} .
$$
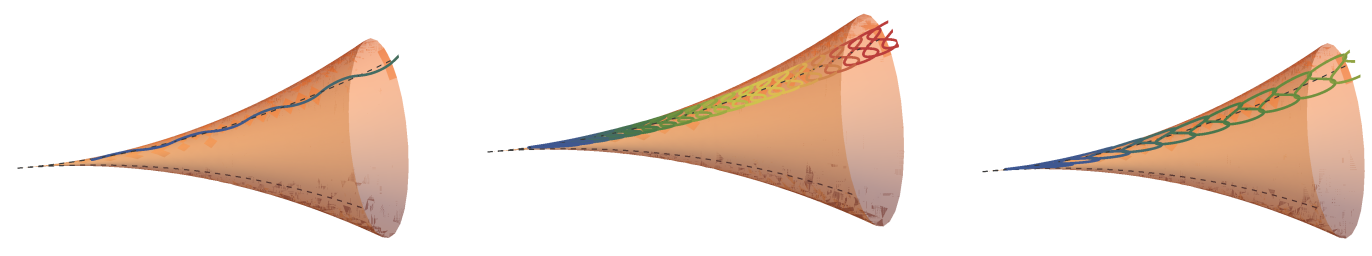

Figure 5.7: Trajectories in the $(z, \chi)$-plane for the infinite linear quiver geometry in eq.(5.51), with initial conditions $p_{\chi}^{(0)}=0, z^{(0)}=1$, and $p_{z}^{(0)}=1$. From left to right we change the initial value of $\chi^{(0)}$ to $0.25,0.90$, and 1.25 respecitvely, corresponding to energies $E \approx 8.95, E \approx 24.94$ and $E \approx 30.08$.

Though the existence of an integrable string embedding is not sufficient to prove integrability, we do not see any signs of chaotic behaviour in the numerical evolution. The strings moves off along the infinite $z$-direction and never returns, resulting in seemingly orderly trajectories.

As these trajectories are non-periodic we can no longer study a Poincaré section of the phase space and hope to expect to see trajectories confined to the surfaces of KAM tori.

This is reminiscent of the linear quivers that have been known be integrable in other $\frac{1}{2}$ and $\frac{1}{4}$ BPS geometries $[132,140]$, see also section 3.7. These infinite linear quiver solutions are in those cases obtained as the non-Abelian T-dual of a more symmetric solutions on which the string is known to be integrable. As the NATD preserves the integrability of the background these infinite linear quivers are therefore known to be integrable. We will comment more about the relation between this infinite linear quiver background and NATD in the next chapter.

\section{Finite Linear Quiver}

To end a finite linear quiver one has to add a flavour group at the end, as is done for the quiver in Example 2 (4.27) in the previous chapter.

We will now show the numerical evolution, Poincaré sections and Lyapunov exponents that indicate this single flavour brane now spoils the ordered trajectory the infinite linear quiver was endowed with. 

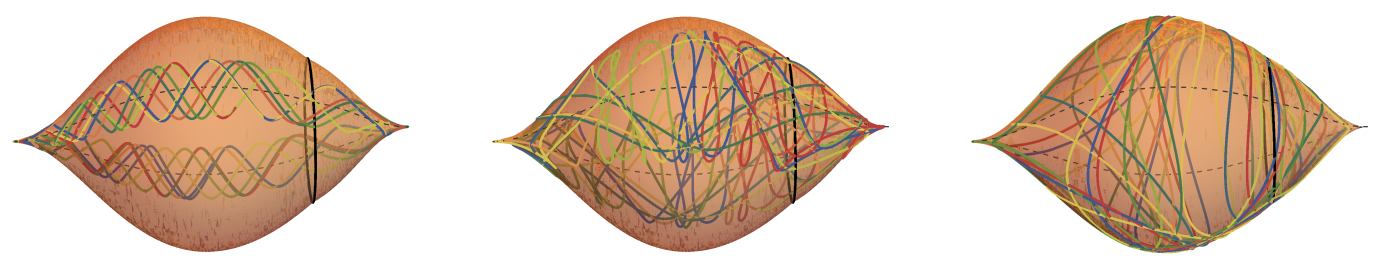

Figure 5.8: Trajectories on the background of Example 2 in (4.27), with all numerical parameters equal to those in figure 5.3. The position of the D8-branes is indicated by the black line.

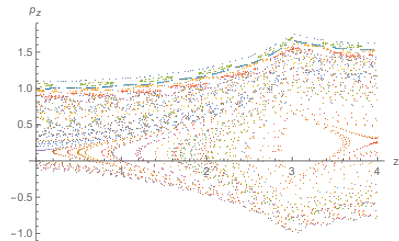

(a) $E=5$

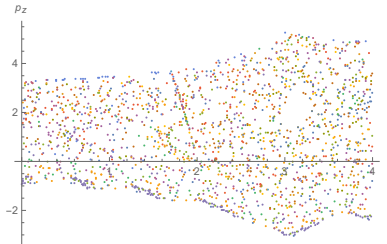

(b) $E=15$

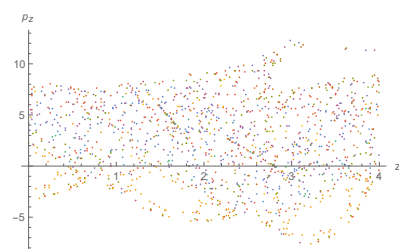

(c) $E=35$

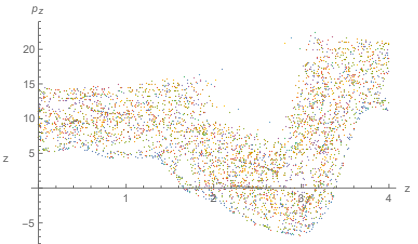

(d) $E=65$

Figure 5.9: Poincaré sections for the $\left(z, p_{z}\right)$-plane at $\chi(t)=0$ at different energie, for the background in Example 2 (5.12), corresponding to the trajectories in figure 5.12.

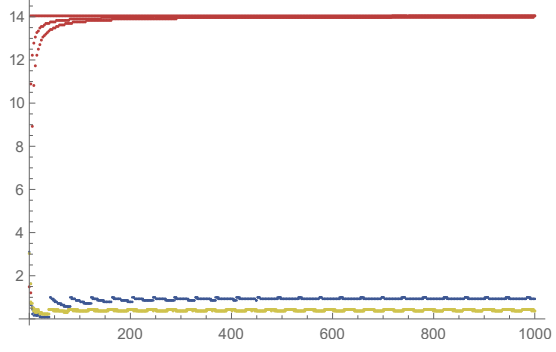

Figure 5.10: Numerical approximations for the largest Lyapunov exponents corresponding to the initial conditions in figue 5.12 in respectively blue, yellow, and red. Using the algorithm in section 5.6. Note that for higher energies the value of the Lyapunov exponent rapidly increases.

\subsubsection{Analysis for the Massless Solution}

The $F_{0}$-flux in the $\mathrm{AdS}_{7}$ supergravity solutions of eq.(4.6) is also known as the Romans mass. In this section we will consider the (non)-integrability of string embeddings that wrap the $\xi$ - 


\section{V. (NON)-INTEGRABILE $6 \mathrm{D} \mathcal{N}=(1,0)$ SCFTS}

direction and move along $\chi$ and $z$ on the massless $\mathrm{AdS}_{7}$ solution, when $F_{0}=0$. We find the trajectories of string on this space to be non-integrable and occasionally flow to a singular point at the end of the geometry, where the dilaton blows up. Since the dilaton is proportional to the string coupling the classical string embeddings we study in the previous section no longer give a good approximation when the strings get close to these points. In the later part of this section we 'complete' this massless $\mathrm{AdS}_{7}$ solution to one of eleven dimensional supergravity, where the $10 \mathrm{~d}$ dilaton is interpreted as the size of the compactified 11-th dimension. The strings then turn into M2-branes wrapping also the eleventh direction. We will study classical toroidal M2-brane configurations on the eleven dimensional completion in the second part of this section. We find this particular sector of classical toroidal M2-branes to be integrable.

We numerically solve the equations of motion for the classical string moving in the $z$ and $\chi$-directions, and wrapping $\xi$. When the Romans mass $F_{0} \sim \alpha^{\prime \prime \prime}=0$, the corresponding function $\alpha(z)$ is

$$
\alpha(z)=-81 \pi^{2} k\left(\frac{1}{2} z^{2}-\frac{2 R_{0}^{2}}{81 \pi^{2} k^{2}}\right)=R^{2}-\mu z^{2} .
$$

Unlike for the piece-wise continuous functions $\alpha(z)$ we considered in the previous section, the rank function in this case is a continuous function as there are no D8-branes on this background. This background only has $k$ D6-branes. Notice that the coordinate range is $|z| \leq \frac{2 R_{0}}{9 \pi k}$. The background is singular at the ends of the space that the dilaton blows up.

\section{Infinite Massless Solution}

From the Kovacic analysis we saw the NVE for $\chi$ has a Liouvillian solution for very large $R_{0}$ (or for very short times). To obtain the corresponding warp factors for the infinite massless solution we expand the $f_{i}(z)$ around $z=0$ and obtain.

$$
f_{1}(z)=8 \pi \sqrt{\frac{R^{2}}{\mu}}, \quad f_{2}(z)=2 \pi \sqrt{\frac{\mu}{R^{2}}}, \quad f_{3}(z)=\frac{\pi}{2} \sqrt{\frac{R^{2}}{\mu}}, \quad f_{4}(z)=-\frac{3 \pi z}{2} .
$$

Again the existence of an integrable string embedding is not sufficient to prove integrability, but we confirm there are not signs of chaotic behaviour in the numerical evolution. As for the infinite linear quiver, the strings move off along the $z$-direction and never return, resulting in orderly trajectories. This indicates that in such a regime of parameters, the string soliton is might be integrable. and possibly the full CFT is integrable in that limit too.

\section{Finite Massless Solution}

Kovacic's analysis (see section 5.3.3) proved inconclusive for a more general value of $R_{0}$. Here we show numerical indicators of chaos, demonstrating the string is in general non-integrable on this massless solution. 

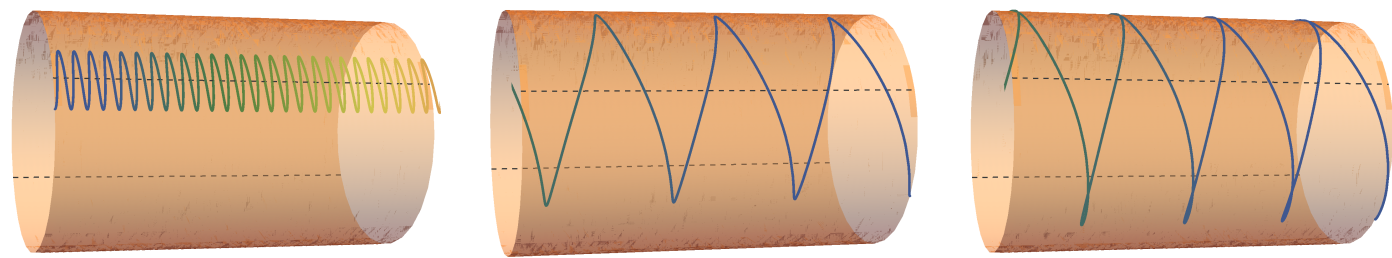

Figure 5.11: Trajectories in the $(z, \chi)$-plane for the infinite massless geometry in eq.(5.53), with initial conditions $p_{\chi}^{(0)}=0, z^{(0)}=0$, and $p_{z}^{(0)}=1$. From left to right we change the initial value of $\chi^{(0)}$ to $0.25,0.90$, and 1.25 respecitvely, corresponding to energies $E \approx 3.70, E \approx 10.04$ and $E \approx 12.09$.
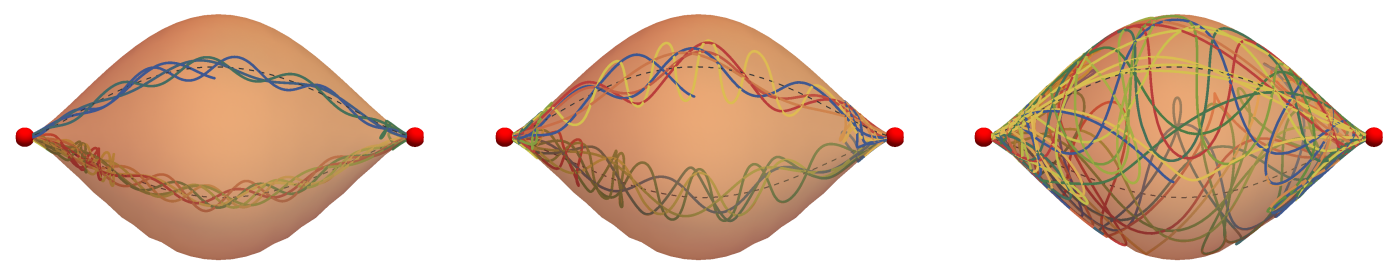

Figure 5.12: Trajectories in the $(z, \chi)$-plane for a string-embedding on the massless backgound with $R=\mu=1$, we only change the initial condition $\chi(0)$, and keep the other initial conditions fixed $p_{\chi}(0)=0.1, z(0)=0, p_{z}(0)=1$. The orange surface represents the the two-sphere fibered with warp-factor $f_{3}(z)$ along the $z$-direction. The dashed line indicated points where $\chi=0, \pi$. Points on opposite sides of this dashed line should be identified since $\chi \in[0, \pi]$. The singularities at the end of the space are indicated by red dots.

To perform the corresponding (numerical) computation on the Lyapunov spectrum, we choose the parameters $k=1, R_{0}=500, \nu=1$, taking as initial conditions for the phase space variables: $t=0, z=0.05, \chi=0.05, p_{t}=100, p_{z}=0.159689, p_{\chi}=0.01$ that satisfy the vanishing of the Hamiltonian. From the plot in Figure 5.13.

This equation is hard to solve exactly, let us distinguish two cases: We observe that for very large values of the parameter $R_{0}$ (or for very short times), the eq. (5.38) reduces to an oscillator equation. This indicates that in such a regime of parameters, the string soliton is might be integrable and possibly the full CFT is integrable in that limit too. 
V. (NON)-INTEGRABILE $6 \mathrm{D} \mathcal{N}=(1,0)$ SCFTS

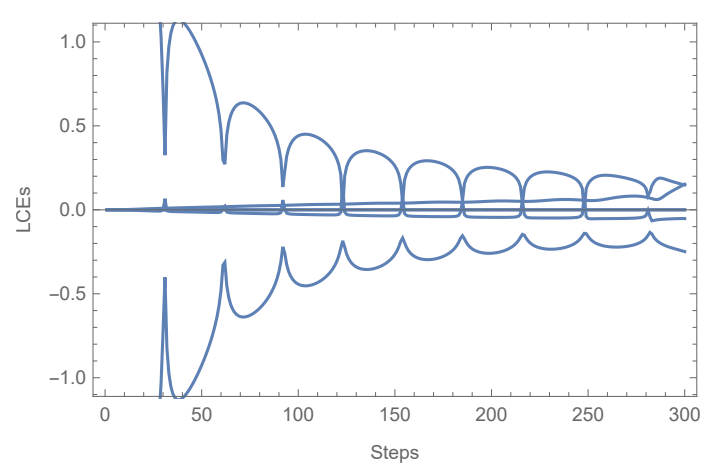

Figure 5.13: LCEs for the massless solution with large $R$.

\section{Uplift to M-theory}

We can uplift the massless solution, using that the 11-dimensional geometry and three-form will be given by

$$
\begin{aligned}
d s_{11}^{2} & =e^{-\frac{2}{3} \phi} g_{\mu \nu} d x^{\mu} d x^{\nu}+e^{\frac{4}{3} \phi}\left(d \beta+A_{\mu} d x^{\mu}\right)^{2} \\
C_{\mu \nu \rho} & =A_{\mu \nu \rho}+B_{\mu \nu} \wedge d \beta
\end{aligned}
$$

where $g_{\mu \nu}$ is the 10-dimensional metric, $A_{\mu}$ the one-form potential, $A_{\mu \nu \rho}$ the three-form potential and $\beta$ is the additional eleventh direction. This gives us for the $\mathrm{AdS}_{7}$ geometries in eq.(4.6) the following result

$$
\begin{aligned}
& d s_{11}^{2}=f_{6}^{-2 / 3}\left[f_{1} d s_{A d S_{7}}^{2}+f_{2} d z^{2}+f_{3} d \Omega^{2}(\chi, \xi)\right]+f_{6}^{4 / 3}\left(d y-f_{5} \cos \chi d \xi\right)^{2} \\
& 6 C_{3}=f_{4} \sin \chi d \chi \wedge d \xi \wedge d y
\end{aligned}
$$

We use the action and constraints for a membrane (see for example [187]),

$$
\begin{aligned}
& S=\int d \tau d \sigma d \rho\left(\gamma_{\tau \tau}+L^{2} \operatorname{det}\left(\gamma_{\alpha \beta}\right)+2 L \epsilon^{i j k} C_{\mu \nu \delta} \partial_{i} X^{\mu} \partial_{j} X^{\nu} \partial_{k} X^{\delta}\right) . \\
& \gamma_{\tau \alpha}=0, \quad \gamma_{\tau \tau}+L^{2} \operatorname{det} \gamma_{\alpha \beta}=0 . \quad(\alpha, \beta=\sigma, \rho)
\end{aligned}
$$

We propose a membrane configuration that is the natural lift of the string soliton we proposed in the main part of the paper,

$$
t=t(\tau), \quad z=z(\tau), \quad \chi=\chi(\tau), \quad \xi=k \sigma, \quad y=\lambda \rho .
$$

We find an effective Lagrangian and constraint that can be written as,

$$
\begin{aligned}
& \mathcal{L}=f_{6}^{-2 s / 3}\left[f_{1} \dot{t}^{2}-f_{2} \dot{z}^{2}-f_{3} \dot{\chi}^{2}+L^{2} k^{2} \lambda^{2} f_{3} f_{6}^{4 s / 3} \sin ^{2} \chi+L k \lambda f_{4} f_{6}^{2 s / 3} \dot{\chi} \sin \chi\right] \\
& 0=-f_{1} \dot{t}^{2}+f_{2} \dot{z}^{2}+f_{3} \dot{\chi}^{2}+L^{2} k^{2} \lambda^{2} f_{3} f_{6}^{4 s / 3} \sin ^{2} \chi
\end{aligned}
$$


One can see that choosing $s=0$ (and identifying $L \lambda k=\nu$ ), this reduces to the effective Lagrangian for the string in eq.(5.6). For $s=1$, we have the full Lagrangian and constraint for the membrane. We now study the equations of motion derived from eq.(5.58) and find,

$$
\begin{aligned}
& \dot{t}=\frac{E}{f_{1}} f_{6}^{2 s / 3}, \\
& 2 f_{3} \ddot{\chi}=-2 L^{2} k^{2} \lambda^{2} f_{3} \cos \chi \sin \chi f_{6}^{4 s / 3}-2 \dot{z} \dot{\chi} f_{3}^{\prime}+2 L k \lambda \sin \chi \dot{z} f_{4}^{\prime} f_{6}^{2 s / 3}+\frac{4 s}{3} \frac{f_{3} f_{6}^{\prime}}{f_{6}} \dot{z} \dot{\chi} . \\
& \ddot{z}+E^{2} \frac{f_{6}^{4 s / 3}}{2 f_{1} f_{2}}\left(\frac{f_{1}^{\prime}}{f_{1}}-\frac{2 s}{3} \frac{f_{6}^{\prime}}{f_{6}}\right)+\dot{z}^{2}\left(\frac{f_{2}^{\prime}}{2 f_{2}}-\frac{s}{3} \frac{f_{6}^{\prime}}{f_{6}}\right)+\dot{\chi}^{2} \frac{f_{3}}{2 f_{2}}\left(-\frac{f_{3}^{\prime}}{f_{3}}+\frac{2 s}{3} \frac{f_{6}^{\prime}}{f_{6}}\right)+ \\
& +L k \lambda \sin \chi \dot{\chi} \frac{f_{4}^{\prime}}{f_{2}} f_{6}^{2 s / 3}+L^{2} k^{2} \lambda^{2} \frac{f_{3} f_{6}^{4 s / 3} \sin ^{2} \chi}{2 f_{2}}\left(\frac{f_{3}^{\prime}}{f_{3}}+\frac{2 s}{3} \frac{f_{6}^{\prime}}{f_{6}}\right)=0 .
\end{aligned}
$$

The reader can check that for $s=0$ the equation of motion of the string are recovered.

We now apply the same algorithmic procedure as before in section 5.3.2. The configuration with $\chi(\tau)=\dot{\chi}(\tau)=\ddot{\chi}(\tau)=0$ solves the $\chi$-equation of motion and leaves the $z$-equation as,

$$
\ddot{z}+E^{2} \frac{f_{6}^{4 s / 3}}{2 f_{1} f_{2}}\left(\frac{f_{1}^{\prime}}{f_{1}}-\frac{2 s}{3} \frac{f_{6}^{\prime}}{f_{6}}\right)+\dot{z}^{2}\left(\frac{f_{2}^{\prime}}{2 f_{2}}-\frac{s}{3} \frac{f_{6}^{\prime}}{f_{6}}\right)=0 .
$$

Calculating explicitly for the function $\alpha(z)=\mu\left(1-z^{2}\right)$ (after choosing constants appropriately), using the explicit expression for $f_{i}(z)$, we find that $z$-equation is solved by

$$
z_{s}(\tau)=\cosh \tau \text {. }
$$

Fluctuating the $\chi$-equation as $\chi(\tau)=0+\epsilon f(\tau)$, we find the NVE,

$$
\begin{aligned}
& \ddot{f}+\mathcal{B} \dot{f}+\mathcal{A} f=0, \\
& \mathcal{B}=\left.\dot{z}(\tau)\left(\frac{f_{3}^{\prime}}{f_{3}}-\frac{2 s}{3} \frac{f_{6}^{\prime}}{f_{6}}\right)\right|_{z_{s}}=2 \operatorname{coth} \tau, \\
& \mathcal{A}=L^{2} k^{2} \lambda^{2} f_{6}^{4 s / 3}-\left.L \lambda k f_{6}^{2 s / 3} \frac{f_{4}^{\prime}}{f_{3}} \dot{z}(\tau)\right|_{z=z_{s}}=n_{1} \sinh \tau+n_{2} \sinh ^{2} \tau .
\end{aligned}
$$

With $n_{1}, n_{2}$ two numbers. In what follows we take $s=1$ to discuss the case of the membrane only. It is convenient to change the variable $v=e^{-\tau}$, to have an NVE that reads,

$$
f^{\prime \prime}+\frac{3 v^{2}+1}{v\left(v^{2}-1\right)} f^{\prime}+\left(\frac{n_{1}}{2 v^{3}}\left(1-v^{2}\right)+\frac{n_{2}}{4 v^{4}}\left(1-v^{2}\right)^{2}\right) f=0 .
$$

We denoted $f^{\prime}=\frac{d f}{d v}$. We can construct the effective potential of the associated Schrödinger problem, as indicated in eq.(5.15),

$$
V(v)=\frac{2 \mathcal{B}^{\prime}+\mathcal{B}^{2}-4 \mathcal{A}}{4}=\frac{3}{4 v^{2}}-\frac{n_{1}}{2 v^{3}}+\frac{n_{1}}{2 v}-\frac{n_{2}}{4 v^{4}}-\frac{n_{2}}{4}+\frac{n_{2}}{2 v^{2}} .
$$




\section{V. (NON)-INTEGRABILE $6 \mathrm{D} \mathcal{N}=(1,0)$ SCFTS}

We observe that the first of the necessary conditions discussed in section 5.3.1 is satisfied. The Kovacic algorithm could produce a Liouvillian solution for the membrane, which could imply the classical membrane configuration in eq.(5.57) is integrable.

We see that the problem with the string is that it 'misses' the effects of the dilaton, represented above by the various powers of $f_{6}^{2 / 3}$. It is the presence of the dilaton (that the 'classical limit' of the Polyakov action misses), which changes the equation to introduce integrability. Note that the dilaton becomes very large at the ends of the interval $z= \pm 1$ (in these units), hence it cannot be neglected.

\section{Integrability of the M2-branes}

We will now demonstrate the brane configuration in eq.(5.57) is indeed integrable. To see this more easily, we perform a coordinate transformation on the background. Using that $\alpha(z)=R^{2}-\mu z^{2}$ we insert this in the expressions for the functions $f_{i}(z)$ and find

$$
f_{6}^{4}(z)=\left(\frac{81 \pi^{5 / 2}}{\sqrt{2}}\right)^{4} \frac{1}{R^{4} \mu^{5}}\left(R^{2}-\mu z^{2}\right)^{3}=e^{4 \phi_{0}} \frac{1}{R^{4} \mu^{5}}\left(R^{2}-\mu z^{2}\right)^{3}
$$

Inserting these functions explicitly in the metric gives us

$$
\begin{array}{r}
d s^{2}=2 \pi e^{-\frac{2}{3} \phi_{0}} \mu^{1 / 3} R^{2 / 3}\left[4 d s_{\mathrm{AdS}_{7}}^{2}+\mu\left(R^{2}-\mu z^{2}\right)^{-1} d z^{2}+\frac{\mu^{3}}{4 R^{2}}\left(R^{2}-\mu z^{2}\right) d \Omega_{2}^{2}(\chi, \xi)\right]+\ldots \\
\ldots+e^{\frac{4}{3} \phi_{0}} \mu^{-5 / 3} R^{-4 / 3}\left(R^{2}-\mu z^{2}\right)\left[d \beta-\frac{\mu}{81 \pi^{2}} \cos \chi d \xi\right]^{2}
\end{array}
$$

We now make a coordinate transformation $z=\frac{R}{\sqrt{\mu}} \sin \nu, \frac{d z^{2}}{R^{2}-\mu z^{2}}=\frac{d \nu^{2}}{\mu}$ and obtain for the metric

$$
\begin{aligned}
d s^{2}=2 \pi e^{-\frac{2}{3} \phi_{0}} \mu^{1 / 3} R^{2 / 3}\left[4 d s_{\mathrm{AdS}_{7}}^{2}+d \nu^{2}+\frac{1}{4} \cos ^{2} \nu\left(\mu^{3} d \Omega_{2}^{2}(\chi, \xi)+\ldots\right.\right. \\
\left.\left.\ldots+\frac{2}{\pi} e^{2 \phi} \mu^{-2}\left[d \beta-\frac{\mu}{81 \pi^{2}} \cos \chi d \xi\right]^{2}\right)\right]
\end{aligned}
$$

We now rescale $d \beta=\frac{\mu}{81 \pi^{2}} d x$ and explicitly insert $e^{2 \phi_{0}}=\frac{1}{2} 81^{2} \pi^{5}$

$$
d s^{2}=2 \pi e^{-\frac{2}{3} \phi_{0}} \mu^{1 / 3} R^{2 / 3}\left[4 d s_{\mathrm{AdS}_{7}}^{2}+d \nu^{2}+\frac{1}{4} \cos ^{2} \nu\left(\mu^{3} d \Omega_{2}^{2}(\chi, \xi)+[d x-\cos \chi d \xi]^{2}\right)\right]
$$

We can remove the prefactor by rescaling all the coordinates by a factor $\sqrt{2 \pi} e^{-\frac{3}{4} \phi_{0}} \mu^{1 / 6} R^{1 / 3}$. At last we have one prefactor $\mu^{3}$ left, that can be absorbed in the $\Omega_{2}^{2}(\chi, \xi)$-term, giving us

$$
d s^{2}=4 d s_{\mathrm{AdS}_{7}}^{2}+d \nu^{2}+\frac{1}{4} \cos ^{2} \nu\left(d \Omega_{2}^{2}\left(\frac{\chi^{\prime}}{K}, \xi^{\prime}\right)+\left[d x^{\prime}-\cos \frac{\chi^{\prime}}{K} d \xi^{\prime}\right]^{2}\right)
$$


We see that the last terms form the four-sphere, with the angle $\chi$ everywhere divided by $K$. The resulting metric is thus $\mathrm{AdS}_{7} \times \mathrm{S}^{4} / \mathbb{Z}_{K}$, where $\chi \in\left[0, \frac{\pi}{K}\right]$.

Inserting the explicit expressions for $f_{4}(z)$ and $f_{5}(z)$ in the three-form and performing the same coordinate transformations, we obtain

$$
F_{4}=\operatorname{vol} \Omega_{4}\left(\nu, \frac{\chi^{\prime}}{K}, \xi^{\prime}, x^{\prime}\right) .
$$

The classical toroidal M2-brane embedding we consider has two of its worldsheet coordinates fixed, as it wraps both the $\beta=\frac{\mu}{81 \pi^{2}} x$ and $\xi$-directions, and does not move along these directions. In the remaining target space directions it will therefore only see two legs of the $F_{4}$, on the $n u=\arcsin \frac{\sqrt{\mu}}{R} z$ and $\chi$-directions.

If we leave out the dependence of the target space on $v$, it will look like a three-sphere with a constant antisymmetric $F_{3}$-flux proportional to the volume of the three-sphere. This is the target space of the classical WZW-model on $S U(2)$, which is known to be integrable. Since the membrane also wraps around the $\xi$-direction, the remaining worldsheet coordinate of the membrane will not depend on it, and will describe a subsector of the WZW-model on $S U(2)$.

It would be very interesting to see if this approach can be generalised to more general M2brane configurations, and if this could somehow be used to show the full membrane worldvolume theory on particular backgrounds likeAdS ${ }_{7} \times S^{4}$ is integrable.

\subsubsection{Vanishing Warp Factor}

In this section, we will study our analytical study of the integrability of the string worldsheet on the background with $\alpha(z)=A \sin \omega z$. We hinted briefly at this possibility in section 5.3.2, where we showed the NVE equations for the string moving along the $\rho$-direction (5.17), and the $\varphi$-direction (5.22), become simple harmonic oscillators when the warp factor $f_{1}(z)$ is equal to a constant. In addition the NVE for the string moving along the $\chi$-direction passed Kovacic's criteria, hinting this particular embedding might also have an analytic solution.

Our numerical analysis indeed confirms the integrability of the string dynamics in the $(z, \chi)$ plane for this background. ${ }^{3}$ In order to distinguish the integrable solution from a non-integrable one we will study the background with $\alpha(z)$ given by

$$
\alpha(z)=-81 \pi^{2}\left[A \sin \left(\frac{\pi z}{4}\right)+B \sin \left(\frac{\pi z}{2}\right)\right],
$$

where we let $B$ range from 0 (for which the dynamics of the string is integrable) to 1 . We will show that the dynamics becomes chaotic as soon as $B$ deviates from 0 .

\footnotetext{
${ }^{3}$ In the next chapter we will prove analytically the strings on this background are indeed integrable. This underlines the reliability of the numerical methods used in this chapter, where we used these same numerical methods to show that the dynamics of strings on backgrounds with piece-wise continuous rank functions were non-integrable.
} 


\section{V. (NON)-INTEGRABILE $6 \mathrm{D} \mathcal{N}=(1,0)$ SCFTS}

In figure 5.14a we start again with an initial value that is very close to the poles of the 2-sphere $(\chi=0$ and $\chi=\pi)$ for the integrable background with $B=0$, the string oscillates as we have seen before for the other backgrounds. As we increase the energy and allow the string to move further away from the poles, it starts to moving freely around the entire 2 -sphere, see figure $5.14 \mathrm{~b}$. Note that even at high energies, the motion of the string remains 'ordered' and quasi-periodic.
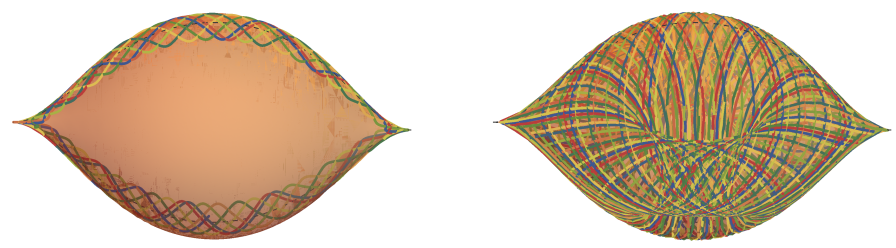

(a) $\chi(0)=0.1, E \approx 6.58$, (b) $\chi(0)=0.9, E \approx 43.33$,

$$
t_{\text {max }}=400, B=0 \quad t_{\max }=400, B=0
$$
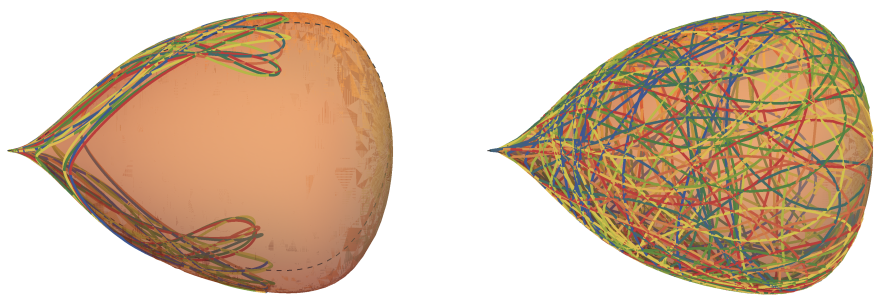

$$
\begin{gathered}
\text { (c) } \chi(0)=0.1, E \approx 7.19,(\mathrm{~d}) \chi(0)=0.9, E \approx 48.95, \\
t_{\max }=150, B=0.2 \\
t_{\max }=250, B=0.2
\end{gathered}
$$

Figure 5.14: Trajectories of the string on the internal space $\mathcal{M}^{3}$ for low and high energies (from left to right). The two images at the top correspond to the integrable background with $B=0$ in eq.(5.71), for those on the bottom $B=0.2$. For the integrable background (in the top two images) the trajectories of the string soliton remain regular, even at high energies. We choose initial conditions $p_{\chi}^{(0)}=0, z^{(0)}=2, p_{z}^{(0)}=1$. For the figures on the left $X^{(0)}=0.1$, for those on th right $\chi^{(0)}=0.9$.

As we go away from $B=0$ we are no longer considering the integrable background. The asymmetry along the $z$-direction of this background makes it harder for the string to probe the 
right side of the space. In figure 5.14c we see that again for low energies the string oscillates around the poles. Though its motion still looks quite regular and quasiperiodic, it already appears more disorderly than the integrable background. We see in figure 5.14d that as we now increase the energy for the string on the non-integrable background, its motion becomes chaotic.

To verify this we obtain the Lyapunov exponents corresponding to our initial conditions. We numerically estimate these largest Lyapunov exponents for the same low and high energy (left to right) initial conditions that we considered in figure 5.14. The result is shown in figure 5.15. We see in figure 5.15a that at low energies the LLE on both backgrounds is comparably small. We expect the LLE for the integrable background ( $B=0$, in red) to asymptote to zero (with some numerical noise). The LLE for the non-integrable background $(B=0.2$, in blue) is a little larger at low energies. This agrees with what we see in figure 5.14a and 5.14c.

We see in figure 5.15b that the dynamics for the high energy string still has an LLE of almost zero on the integrable background $(B=0$, in red), thus numerically confirming the absence of chaos for this case. On the non-integrable background ( $B=0.2$, in blue) the value clearly asymptotes to a non-zero value $\lambda \approx 0.01$, confirming its dynamics is chaotic. This agrees with what we see in figure $5.14 \mathrm{~b}$ and $5.14 \mathrm{~d}$.

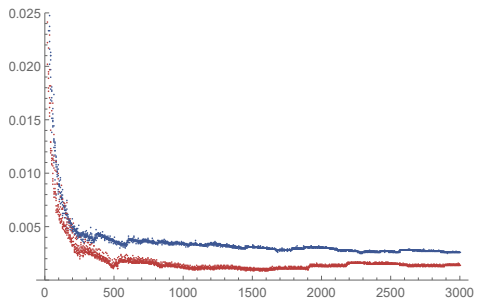

(a) LLE for $\chi(0)=0.1$.

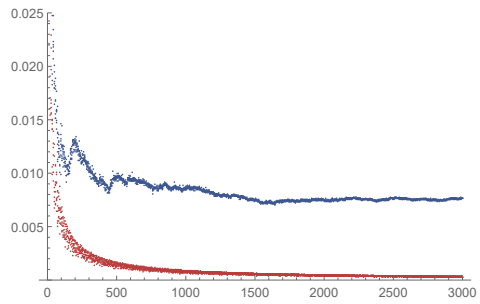

(b) LLE for $\chi(0)=0.9$.

Figure 5.15: Lyapunov exponents for low- (left) and high-energy (right) string configurations, using the same initial conditions as in figure ??. We consider both the integrable background ( $B=0$, in red) and on a non-integrable background $(B=0.2$, in blue). We find that the integrable background has an LLE that asymptotes to zero (indicating the absence of chaotic behaviour), while for the non-integrable background it asymptotes to a finite value, indicating increasingly chaotic dynamics for higher energies. This agrees with what we see in figure 5.14.

To generate the Poincaré sections we choose different initial conditions in the $\left(\chi, p_{z}\right)$-plane, all corresponding to the same energy (5.48). We run the numerical evolution for these initial points and monitor the $\left(z, p_{z}\right)$-plane every time the trajectories pass through the point $\chi(t)=0$.

In figure 5.16a we see that for the integrable background $(B=0)$ the trajectories of the system are confined to the surfaces of embedded KAM tori in the $\left(z, p_{z}, \chi, p_{\chi}\right)$ phase-space. 


\section{V. (NON)-INTEGRABILE $6 \mathrm{D} \mathcal{N}=(1,0)$ SCFTS}

As we increase the value of $B$ we lose the integrability of the dynamical system. This onset of chaos can clearly be seen in figure 5.16b-5.16d, as more and more KAM tori break apart when we increase $B$ until there is no structure left and we have a purely chaotic system for all initial conditions.

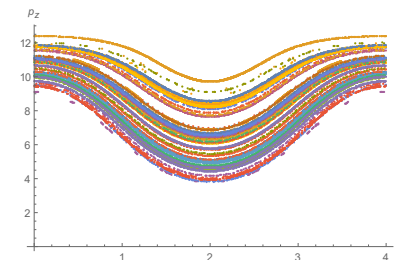

(a) $B=0$

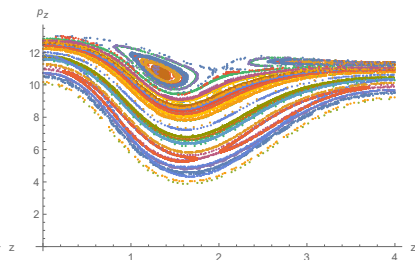

(b) $B=0.025$

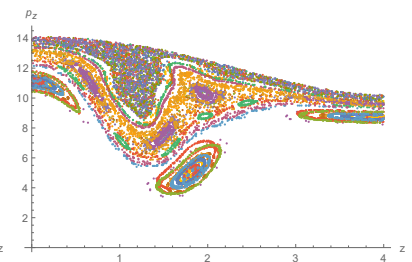

(c) $B=0.050$

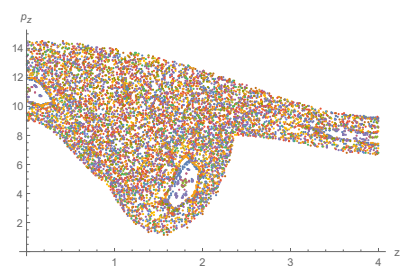

(d) $B=0.065$

Figure 5.16: Poincaré sections for $\left(z, p_{z}\right)$-plane at $\chi(t)=0$, for high energy string configurations $(E=45)$ on backgrounds with values of $B$. As we increase $B$, we clearly see the onset of chaos as more and more KAM tori break apart until there is no structure left and we have a purely chaotic system. 


\subsection{Appendix: Numerical Computation of the Lyapunov Spec- trum}

In this section we briefly discuss the algorithm used to compute the Lyapunov characteristic exponents (LCEs) for a generic system, following [189, 183]. Let us consider a generic ndimensional dynamical system

$$
\dot{q}=V(q),
$$

where $q(\tau)$ is the $n$-dimensional state vector $q=(\vec{X}(\tau), \vec{P}(\tau))$ at time $\tau, \dot{q}=\frac{d q}{d \tau}$ and $V$ is a vector field on the phase space, which generates a flow $f$ :

$$
\dot{f}^{\tau}(q)=V\left(f^{\tau}(q)\right)
$$

where $f^{\tau}(q)=f(q, \tau)$. Consider the evolution under the flow of two nearby points in the phase space, $q_{0}$ and $q_{0}+\delta_{0}$, being $\delta_{0}$ a small perturbation of the initial point $q_{0}$. After a time $\tau$, the perturbation $\delta_{\tau}$ will become:

$$
\delta_{\tau} \equiv f^{\tau}\left(q_{0}+\delta_{0}\right)-f^{\tau}\left(q_{0}\right) \approx D_{q_{0}} f^{\tau}\left(q_{0}\right) \cdot \delta_{0}
$$

The average exponential rate of divergence (or convergence) of two trajectories is then defined by:

$$
\lambda\left(q_{0}, \delta_{0}\right)=\lim _{\tau \rightarrow \infty} \frac{1}{\tau} \log \frac{\left\|\delta_{\tau}\right\|}{\left\|\delta_{0}\right\|}=\lim _{\tau \rightarrow \infty} \frac{1}{\tau} \log \left\|D_{q_{0}} f^{\tau}\left(q_{0}\right) \cdot \delta_{0}\right\|
$$

with $\|\delta\|$ the length of the vector $\delta$. If $\lambda(x, u)>0$, we have exponential divergence of nearby orbits.

The Lyapunov characteristic exponents (LCEs) of order $p, 1 \leq p \leq n$, are introduced to describe the mean rate of growth of a p-dimensional volume in the tangent space. Considering a parallelepiped $U_{0}$ in the tangent space whose edges are the $p$ vectors $\delta_{1}, \ldots, \delta_{p}$, the LCEs of order $p$ are defined by:

$$
\lambda^{p}\left(q_{0}, U_{0}\right)=\lim _{\tau \rightarrow \infty} \frac{1}{\tau} \log \left[\operatorname{Vol}^{p}\left(D_{q_{0}} f^{\tau}\left(U_{0}\right)\right)\right]
$$

being $\mathrm{Vol}^{p}$ the p-dimensional volume defined in the tangent space. It can be seen [188] that there exist $p$ linearly independent vectors $u_{1}, \ldots, u_{p}$ satisfying:

$$
\lambda^{p}\left(q_{0}, U_{0}\right)=\lambda_{1}+\ldots+\lambda_{p}
$$

The tangent vector $\delta_{\tau}$ defined in eq.(5.74) evolves in time satisfying:

$$
\dot{\Phi}_{\tau}\left(q_{0}\right)=D_{q} V\left(f^{\tau}\left(q_{0}\right)\right) \cdot \Phi_{\tau}\left(q_{0}\right), \quad \Phi_{0}\left(q_{0}\right)=I
$$


where $\Phi_{\tau}\left(q_{0}\right)=D_{q_{0}} f^{\tau}\left(q_{0}\right)$. To calculate the trajectory, we have to integrate the system:

$$
\left\{\begin{array}{c}
\dot{q} \\
\dot{\Phi}
\end{array}\right\}=\left\{\begin{array}{c}
V(q) \\
D_{q} V(q) \cdot \Phi
\end{array}\right\}, \quad\left\{\begin{array}{c}
q\left(\tau_{0}\right) \\
\Phi\left(\tau_{0}\right)
\end{array}\right\}=\left\{\begin{array}{c}
q_{0} \\
I
\end{array}\right\}
$$

To compute the spectrum of LCEs, we will use the algorithm discussed in [189], based on the calculation of the order- $p$ LCEs defined in eq.(5.77) and on a repeated application of a Gram-Schmidt orthonormalization procedure (which avoids technical difficulties that arise in the implementation of the recipe described in [188]) that we briefly summarize here.

Recall that if we compute an orthonormal set of vectors $\left\{\hat{\delta}_{i}\right\}$ out of the original set of vectors $\left\{\delta_{i}\right\}$, by using the Gram-Schmidt orthogonalisation procedure, the volume of the parallelepiped spanned by $\delta_{1}, \ldots, \delta_{p}$ is

$$
\operatorname{Vol}\left\{\delta_{1}, \ldots, \delta_{p}\right\}=\left\|\hat{\delta}_{1}\right\| \ldots\left\|\hat{\delta}_{p}\right\|
$$

The algorithm starts by choosing an initial condition $q_{0}$ and a $n \times n$ matrix $\Delta_{0}=\left[\delta_{1}^{0}, \ldots, \delta_{n}^{0}\right]$. Using the Gram-Schmidt procedure, we calculate the corresponding matrix of orthonormal vectors $\hat{\Delta}_{0}=\left[\hat{\delta}_{1}^{0}, \ldots, \hat{\delta}_{n}^{0}\right]$ and integrate eq.(5.79) from $\left\{q_{0}, \Delta_{0}\right\}$ for a short interval $T$, to obtain $q_{1}=f^{T}\left(q_{0}\right)$ and

$$
\Delta_{1} \equiv\left[\delta_{1}^{1}, \ldots, \delta_{n}^{1}\right]=D_{q_{0}} f^{T}\left(\Delta_{0}\right)=\Phi_{T}\left(q_{0}\right) \cdot\left[\delta_{1}^{0}, \ldots, \delta_{n}^{0}\right]
$$

The algorithm proceeds by repeating this integration-orthonormalization procedure $K$ times. During the $k$-th step, the $p$-dimensional volume $\mathrm{Vol}^{p}$ defined in eq.(5.76) increases by a factor of $\left\|w_{1}^{k}\right\| \ldots\left\|w_{p}^{k}\right\|$, where $\left\{w_{1}^{k}, \ldots, w_{p}^{k}\right\}$ is the set of orthogonal vectors calculated from $U_{k}$ using the Gram-Schmidt technique. Then:

$$
\lambda^{p}\left(q_{0}, \Delta_{0}\right)=\lim _{k \rightarrow \infty} \frac{1}{k T} \sum_{i=1}^{k} \log \left(\left\|\hat{\delta}_{1}^{i}\right\| \ldots\left\|\hat{\delta}_{p}^{i}\right\|\right)
$$

From which we can derive

$$
\lambda_{p}=\lim _{k \rightarrow \infty} \frac{1}{k T} \sum_{i=1}^{k} \log \left\|\hat{\delta}_{p}^{i}\right\|
$$

To obtain the Lyapunov spectrum, we continue calculating the quantities:

$$
\frac{1}{K T} \sum_{i=1}^{K} \log \left\|\hat{\delta}_{1}^{i}\right\| \approx \lambda_{1}, \ldots, \frac{1}{K T} \sum_{i=1}^{K} \log \left\|\hat{\delta}_{1}^{n}\right\| \approx \lambda_{n}
$$

for a suitable value of $T$, until they show convergence. 
VI. INTEGRABLE STRINGS ON $\mathrm{ADS}_{7} \times\left(S^{3}\right)_{\lambda}$

\section{Chapter 6}

\section{Integrable strings on $\mathbf{A d S}_{7} \times\left(S^{3}\right)_{\lambda}$}

In the previous chapter we studied the non-integrability of string embeddings on the various $\mathrm{AdS}_{7}$ backgrounds corresponding to general $\mathcal{N}=(1,0)$ SCFTs. Using both analytical and numerical methods we showed that strings on $\mathrm{AdS}_{7}$ backgrounds with a finite number of flavour branes are non-integrable.

In this section we will focus on one particular quiver background where the rank function is continuous (instead of piece-wise continuous), and corresponds to an $\mathrm{AdS}_{7}$ background with an infinite number of flavour branes that are smeared in a particular way. The rank function for this background is of the form $R(z)=A \sin \omega z$. We briefly hinted at this case in the previous chapter (in section 5.3.2), where we showed the NVE equations for the string moving along the $\rho$-direction (5.17), and the $\varphi$-direction (5.22), become simple harmonic oscillators when the warp factor $f_{1}(z)$ is constant. We showed in section 5.5.4 that the numerics of string embeddings in this space suggests the bosonic sector of the string might be integrable.

Since the warp factor $f_{1}(z)$ in eq.(4.7) is of the form

$$
f_{1}(z)=8 \sqrt{2} \pi \sqrt{-\frac{\alpha}{\alpha^{\prime \prime}}}
$$

we see that $f_{1}(z)=$ constant, together with the condition that $\alpha(z)$ has to vanish at the beginning and end of the $z$-interval implies

$$
\alpha(z)=A \sin \omega z .
$$

We will study this particular background in more detail in this chapter:

- In section 6.1 we give the full Massive Type IIA geometry for $\alpha(z)=A \sin \omega z$.

- In section 6.2 we show explicitly that the equations of motion of the bosonic sector of the string worldsheet on this space are integrable and can be derived from a Lax pair. 
- In section 6.3 we discuss the relation of this background with the lambda deformation of $\mathrm{AdS}_{7} \times S^{3}$.

- In section 6.5 we address the peculiar fact that the rank function for this background is smooth and continuous. We show this implies an infinite quiver with all colour nodes proportional to the flavour nodes in the dual SCFT. We show in addition that one can generalise the class of half-BPS $\mathrm{AdS}_{7}$ solutions to include all possible continuous concave rank functions (the same idea can also be applied to the other half-BPS $\mathrm{AdS}_{p+1}$ geometries we introduced in section 3.6).

- Another way of thinking about these continuous rank functions is that the flavour branes on the AdS geometry are 'smeared'. In section 6.6 we approach the idea of continuous rank functions from this angle, and show this leads to the same conclusions as in the previous section.

- In section 6.7 we discuss the field theory interpretation of the dual $6 \mathrm{~d} \mathcal{N}=(1,0) \mathrm{SCFT}$ in more detail. We suggest the dual $6 \mathrm{~d}$ theory is in the large- $N$ limit with the Chern-Simons level $k \rightarrow \infty$. We continue by calculating various observables for the theory.

\section{1 $\quad \mathrm{AdS}_{7}$ Geometry for $\alpha(z)=A \sin \omega z$}

Let us first write the complete Massive IIA solution for $\alpha(z)=A \sin \omega z$. The $z$-coordinate varies in the interval $0 \leq z \leq \frac{\pi}{\omega}$. We choose $\omega=\frac{\pi}{N_{5}}$, being $N_{5}$ a large integer number. The full background of eqs.(4.6), (4.7) then reads,

$$
\begin{array}{ll}
d s^{2}=\frac{\sqrt{2} \pi}{\omega}\left(8 A d S_{7}+\omega^{2} d z^{2}+\left(\frac{\sin ^{2} \omega z}{1+\sin ^{2} \omega z}\right) d \Omega_{2}\right) \\
e^{-2 \phi}=e^{-2 \phi_{0}}\left(1+\sin ^{2} \omega z\right), & B_{2}=\pi\left(-z+\frac{\sin \omega z \cos \omega z}{\omega\left(1+\sin ^{2} \omega z\right)}\right) d \Omega_{2}, \\
F_{0}=\frac{A \omega^{3} \cos \omega z}{162 \pi^{3}}, & F_{2}=-\frac{A \omega^{2}}{81 \pi^{2}}\left(\frac{\sin ^{3} \omega z}{1+\sin ^{2} \omega z}\right) d \Omega_{2} .
\end{array}
$$

The continuous expression for $F_{0}$ now suggests that we have a continuous distribution of D8branes. Indeed, in contrast with the examples discussed in the previous chapters, $F_{0}$ in eq.(6.5) is a continuous function, instead of a piece-wise constant and discontinuous one that is characteristic of localised D8-branes. We postpone the discussion of this to section 6.5. The Ricci scalar on this geometry reads

$$
R=\frac{\omega \sin ^{4} \omega z}{4 \sqrt{2} \pi}\left(\frac{12+100 \cot ^{2} \omega z+75 \cot ^{4} \omega z}{1+\sin ^{2} \omega z}\right)
$$

Notice the background is non-singular. 
VI. INTEGRABLE STRINGS ON $\mathrm{ADS}_{7} \times\left(S^{3}\right)_{\lambda}$

\subsection{Integrability of the Bosonic String}

We will now present a Lax connection for the Polyakov action of the bosonic string on this background, with an Neveu-Schwarz sector given by eqs.(6.3)-(6.4).

As discussed above, the warp-factor $f_{1}(z)$ as defined in eq.(4.6) is constant for this background, making the geometry in eq.(6.3) a direct product of the $\mathrm{AdS}_{7}$ and internal $\mathcal{M}^{3}$ spaces. This simplifies defining a Lax connection for the string on this background considerably as the oscillations of the string on these different spaces decouple. We can write the Polyakov action in eq.(5.1), for the string on this background as the sum of the action for a string on a seven-dimensional $\mathrm{AdS}_{7}$ geometry, and the action for the string on the three-dimensional internal space $\mathcal{M}^{3}$, with a $B_{2}$-field.

$$
\begin{aligned}
S_{\text {Polyakov }} & =S_{\text {Polyakov }}^{\mathrm{AdS}_{7}}+S_{\text {Polyakov }}^{\mathcal{M}^{3}} \\
= & -\frac{1}{4 \pi \alpha^{\prime}} \int_{\Sigma} d^{2} \sigma \eta^{a b} G_{\alpha \beta}^{\mathrm{AdS}_{7}} \partial_{a} X^{\alpha} \partial_{b} X^{\beta} \\
& -\frac{1}{4 \pi \alpha^{\prime}} \int_{\Sigma} d^{2} \sigma\left(\eta^{a b} G_{\mu \nu}^{\mathcal{M}^{3}}+\epsilon^{a b} B_{\mu \nu}^{\mathcal{M}^{3}}\right) \partial_{a} X^{\mu} \partial_{b} X^{\nu},
\end{aligned}
$$

where the Latin indices range over the worldsheet coordinates, and the Greek indices range over the target space. In particular, $\alpha, \beta$ range over the $\mathrm{AdS}_{7}$ directions, and $\mu, \nu$ range over the coordinates $z, \chi$ and $\xi$ of the internal space $\mathcal{M}^{3}$.

Since first part of this action can be written as a principal chiral model (PCM) on the $\mathbb{Z}_{2}$ graded symmetric coset $A d S_{7} \cong S O(2,6) / S O(1,6)$ we can simply obtain a Lax connection using the coset constructions defined in chapter 2

Next we will turn our attention to a Lax connection whose flatness condition gives the equations of motion for the strong on the internap space $\mathcal{M}^{3}$, as can be derived from the action $S_{P}^{\mathcal{M}^{3}}$ in eq.(6.7). We will elaborate more on the derivation of this Lax connection in Section 6.3. The Lax connection is of the form

$$
\mathcal{L}_{ \pm}=2(1+\sqrt{2}) \frac{A_{ \pm}}{1 \mp Z}, \quad \text { where } \quad A_{ \pm}=\left(\begin{array}{c}
A_{ \pm}^{1} \\
A_{ \pm}^{2} \\
A_{ \pm}^{3}
\end{array}\right)
$$


and

$$
\begin{aligned}
A_{ \pm}^{1}= & \pm \sin \chi \sin \xi z_{ \pm} \pm \frac{\sin 2 z}{2\left(1+\sin ^{2} z\right)}\left(\cos \chi \sin \xi \chi_{ \pm}+\sin \chi \cos \xi \xi_{ \pm}\right) \\
& -\frac{\sin ^{2} z}{\sqrt{2}\left(1+\sin ^{2} z\right)}\left(2 \cos \xi \chi_{ \pm}-\sin 2 \chi \sin \xi \xi_{ \pm}\right) \\
A_{ \pm}^{2}= & \mp \sin \chi \cos \xi z_{ \pm} \pm \frac{\sin 2 z}{2\left(1+\sin ^{2} z\right)}\left(\cos \chi \cos \xi \chi_{ \pm}-\sin \chi \sin \xi \xi_{ \pm}\right) \\
& -\frac{\sin ^{2} z}{\sqrt{2}\left(1+\sin ^{2} z\right)}\left(2 \sin \xi \chi_{ \pm}+\sin 2 \chi \cos \xi \xi_{ \pm}\right) \\
A_{ \pm}^{3}= & \mp \cos \chi z_{ \pm}+\frac{\sin \chi}{2\left(1+\sin ^{2} z\right)}\left( \pm \sin 2 z \chi_{ \pm}+2 \sqrt{2} \sin ^{2} z \sin \chi \xi_{ \pm}\right)
\end{aligned}
$$

We denoted by $Z$ the spectral parameter, and use the notation $f_{+}=\partial_{+} f, f_{-}=\partial_{-} f$ and $f_{+-}=f_{-+}=\partial_{+} \partial_{-} f$. One can check that the flatness condition for this connection is equivalent to the equations of motion for the Polyakov action on the internal space $\mathcal{M}^{3}$ with $B_{2}$-field included. These equations of motion take the form,

$$
\begin{aligned}
z_{+-}= & \frac{1}{2 \sqrt{2}\left(\sin ^{2} z+1\right)^{2}}\left(\sqrt{2} \sin 2 z\left(\chi_{+} \chi_{-}+\sin ^{2} \chi \xi_{+} \xi_{-}\right)\right. \\
& \left.-\left(5+\sin ^{2} z\right) \sin ^{2} z \sin \chi\left(\chi_{+} \xi_{-}-\chi_{-} \xi_{+}\right)\right) \\
\chi_{+-}= & \frac{1}{2} \sin 2 \chi \xi_{+} \xi_{-}+\frac{1}{2 \sqrt{2}}\left(1+\frac{4}{1+\sin ^{2} z}\right) \sin \chi\left(z_{+} \xi_{-} z_{-} \xi_{+}\right) \\
& -\frac{\cot z}{1+\sin ^{2} z}\left(z_{+} \chi_{-}+z_{-} \chi_{+}\right) \\
\xi_{+-}= & -\frac{1}{2 \sqrt{2}}\left(1+\frac{4}{1+\sin ^{2} z}\right) \sin ^{-1} \chi\left(z_{+} \chi_{-}-z_{-} \chi_{+}\right) \\
& -\frac{\cot z}{1+\sin ^{2} z}\left(z_{+} \xi_{-}+z_{-} \xi_{+}\right)-\frac{\cos \chi}{\sin \chi}\left(\chi_{+} \xi_{-}+\chi_{-} \xi_{+}\right) .
\end{aligned}
$$

It is illuminating to discuss the deeper reason behind the integrability of this background. We will do so in the next section.

\subsection{Relation with $\lambda$-deformation}

In this section we will give a further explanation for the integrability of the string worldsheet on the background (6.3)-(6.5), and derive the Lax pair that we stated in the previous section.

It turns out that the Neveu-Schwarz sector part of the internal space $\mathcal{M}^{3}$ for this backgroundeqs.(6.3)-(6.4) is exactly equal to that of the $\lambda$-deformed Wess Zumino Witten (WZW) model 
VI. INTEGRABLE STRINGS ON $\mathrm{ADS}_{7} \times\left(S^{3}\right)_{\lambda}$

on $\mathrm{SU}(2)$. We start from the WZW model, which is given by an action of the form,

$$
S_{W Z W, k}=\frac{k}{2 \pi} \int_{\partial \mathcal{B}} d^{2} \sigma \operatorname{Tr}\left[J_{a} J^{a}\right]+\frac{k}{6 \pi} \int_{\mathcal{B}} d^{3} \sigma \epsilon_{a b c} \operatorname{Tr}\left[J^{a} J^{b} J^{c}\right] .
$$

The $\lambda$-deformation is an integrable deformation of the WZW model [141]. Namely, a deformation term is added to the action in eq.(6.9) that preserves the integrability. The action of the $\lambda$-deformed WZW model is given by

$$
S_{\lambda}=S_{W Z W, k}+\frac{k}{\pi} \int_{\partial \mathcal{B}} d^{2} \sigma \tilde{J}_{+}^{A}\left(\lambda^{-1}-D^{T}\right)_{A B}^{-1} j_{-}^{B}
$$

where $J_{a}$ as before in eq.(6.9) is the left invariant current, and $\tilde{J}_{a}=\partial_{a} g g^{-1}$ is the right invariant current as we defined earlier above eq.(2.20). Notice these currents are algebra valued and the indices $A$ and $B$ range over the components of the algebra of the group on which we study this action. The matrix $D_{A B}=\operatorname{Tr}\left[T_{A} g T_{B} g^{-1}\right]$ relates the left and right invariant currents as $J_{a}^{A}=D_{B}^{A} \tilde{J}_{a}^{B}$. Here the $T_{A}$ are the generators of the group.

If we analyse the $\lambda$-deformed WZW model on the Lie group SU(2) [142], the action (6.10) is equivalent to the Polyakov action of the string on a target space of the form,

$$
\begin{aligned}
d s_{\lambda}^{2} & =2 k\left(\frac{1+\lambda}{1-\lambda} d z^{2}+\frac{1-\lambda^{2}}{\Delta} \sin ^{2} z d \Omega_{2}^{2}\right), \\
B_{2}^{\lambda} & =-2 k\left(z-\frac{(1-\lambda)^{2}}{\Delta} \cos z \sin z\right) \operatorname{vol} \Omega_{2}, \\
e^{-2 \Phi^{\lambda}} & =e^{-2 \Phi_{0}} \Delta,
\end{aligned}
$$

where $\Delta=1+\lambda^{2}-2 \lambda \cos 2 z$ and $\lambda \in[0,1]$. For $\lambda=0$ we obtain the original WZW model. The action we obtain for $\lambda \rightarrow 1$ is related to the non-Abelian T-dual of the WZW model in eq.(6.9). See [142] for a detailed explanation.

The $\lambda$-deformation for $\lambda=3-2 \sqrt{2}$

The connection between the $\lambda$-deformation of the WZW model on SU(2) in eq.(6.11) and the background solution in eqs.(6.3)-(6.5) is made by noting that for $\lambda=3-2 \sqrt{2}$, which implies $\Delta=4 \lambda\left(1+\sin ^{2} z\right)$, the geometry of eq.(6.11) reads,

$$
\begin{aligned}
d s_{\lambda}^{2} & =2 \sqrt{2} k\left(d z^{2}+\frac{\sin ^{2} z}{1+\sin ^{2} z} d \Omega_{2}^{2}\right), \\
B_{2}^{\lambda} & =-2 k\left(z-\frac{\sin z \cos z}{1+\sin ^{2} z}\right) \operatorname{vol} \Omega_{2}, \\
e^{-2 \Phi^{\lambda}} & =e^{-2 \Phi_{0}^{\lambda}}(12-8 \sqrt{2})\left(1+\sin ^{2} z\right) .
\end{aligned}
$$


This is identical to the Neveu-Schwarz sector of the internal space $\mathcal{M}^{3}$ in eqs.(6.3), (6.4) if we identify $\omega=\frac{\pi}{2 k}$ and choose conveniently $e^{-2 \Phi_{0}^{\lambda}}$. The holographic limit $\omega \rightarrow 0$ associated to long quivers, corresponds to $k \rightarrow \infty$, the semi-classical limit of the WZW model. We write the metric, dilaton and $B_{2}$-field for this solution as,

$$
d s_{10}^{2}=8 \sqrt{2} \pi d s_{\mathrm{AdS}_{7}}^{2}+\frac{\pi}{2} d s_{\lambda}^{2}, \quad e^{-2 \Phi}=e^{-2 \Phi^{\lambda}}, \quad B_{2}=\pi B_{2}^{\lambda} .
$$

In summary, for the function $\alpha(z)=A \sin (\omega z)$, the geometry becomes a direct product of $\mathrm{AdS}_{7} \times \mathcal{M}^{3}$. The sigma model for the string factorises into a sigma model on $\mathrm{AdS}_{7}$ times a sigma model on $\mathcal{M}^{3}$ coupled to a $B_{2}$-field. The first is integrable, and a Lax pair can be written as explained in detail in section 2.2.1. The sigma model on $\mathcal{M}^{3}$ is the $\lambda$-deformation of the WZW model on $S^{3}$ - see [142] - for a particular value of the parameter $\lambda=3-2 \sqrt{2}$. This implies the existence of a Lax pair, given in eq.(6.8), for this part of the space. As a consequence, the Neveu-Schwarz sector of the string sigma model on the whole solution of eqs.(6.3),(6.5) is integrable.

\subsubsection{Relation with the NATD of $\operatorname{AdS}_{7} \times \mathrm{S}^{3}$}

When we expand the Massive IIA solution of eqs.(6.3)- (6.4) near $z \rightarrow 0$ we find,

$$
\begin{aligned}
& d s^{2} \sim 8 \pi \frac{\sqrt{2}}{\omega} A d S_{7}+\sqrt{2} \pi \omega\left(d z^{2}+z^{2} d \Omega_{2}\right), \\
& e^{-2 \phi} \sim e^{-2 \phi_{0}}\left(1+\omega^{2} z^{2}\right), \quad B_{2} \sim-\frac{5 \pi \omega^{2}}{3} z^{3} d \Omega_{2}, \\
& F_{2} \sim-\frac{A \omega^{5}}{81 \pi^{2}} z^{3} d \Omega_{2}, \quad F_{0} \sim-\frac{A \omega^{3}}{162 \pi^{2}} .
\end{aligned}
$$

which would be a background for an the infinite linear quiver as we studied in section 5.5.2.

It has been shown that in the other half-BPS $\mathrm{AdS}_{p+1}$ backgrounds, the only solution on which the string is integrable is typically this infinite linear quiver, which can in those cases be obtained as a NATD of a more symmetric solution. For the $\mathrm{AdS}_{7}$ case the NATD of $\mathrm{AdS}_{7} \times \mathrm{S}^{3}$ is however not equal to this infinite linear quiver background, but is instead of the form

$$
\begin{aligned}
& d s^{2}=L_{A d S}^{2} A d S_{7}+L^{2}\left(d r^{2}+\frac{r^{2}}{r^{2}+1} d \Omega_{2}\right), \\
& e^{-2 \phi}=e^{-2 \phi_{0}}\left(1+r^{2}\right), \quad B_{2}=\mu_{0} \frac{r^{3}}{1+r^{2}} d \Omega_{2}, \quad F_{2}=\nu_{0} \frac{r^{3}}{1+r^{2}} z^{3} d \Omega_{2}, \quad F_{0}=f_{0} .
\end{aligned}
$$

Where $\mu_{0}, \nu_{0}, L_{A d S}, L, f_{0}, \phi_{0}$ are constants.

It would be interesting to see if we can think about this infinite linear quiver background as obtained by non-Abelian T-duality applied on some other 'seed-solution'. This will however not be $\mathrm{AdS}_{7} \times \mathrm{S}^{3}$ but could perhaps be a more complicated background. 
VI. INTEGRABLE STRINGS ON $\mathrm{ADS}_{7} \times\left(S^{3}\right)_{\lambda}$

\subsection{The Type IIA Superstring on $\operatorname{AdS}_{7} \times\left(S^{3}\right)_{\lambda}$}

This analysis has been postponed to a forthcoming publication.

\subsection{Continuous Rank Functions and Infinite Quivers}

As we pointed out in section 6.1, the integrable string background has a continuous function $F_{0}(z)$. In this the solution is different from the ones with piece-wise continuous rank functions, that we considered in the previous chapter. In this section we will show such continuous rank functions can be thought of as corresponding to infinite linear quivers.

The supergravity description is only valid for SCFTs with very long quivers. This makes it difficult to perform calculations on both the SCFT side and compare them with supergravity calculations. In section 3.5.2 we defined the conventional approach of obtaining long quivers, by 'scaling' a short quiver to one for which the supergravity description is valid. He we will show how by instead first defining an arbitrary number of flavour groups $F_{i}$, and using the consistency condition to then fix the ranks of all the gauge groups, one can define a quiver of arbitrary length. In the limit $N / F \rightarrow \infty$ this gives rise to continuous rank functions. We will illustrate this in detail for two particular cases, a parabolic rank function (section 6.5.2), and a sinusoidal rank function (section 6.5.3).

\subsubsection{Defining Quivers from their Flavour Groups}

We do this by defining first all of the flavour groups $F_{k} \geq 0$, where $k$ is an arbitrary number giving the length of this quiver.

Once all the flavour groups have been defined, the ranks of the gauge groups are fixed by the consistency condition of eq.(3.24),

$$
2 N_{k}-N_{k+1}-N_{k-1}=F_{k} .
$$

The quiver starts with $N_{0}=0$, and $N_{1}=n$, where $n$ is some arbitrary number for the first gauge group in the quiver. Inserting this in the above condition then dictates the rank of the next gauge group

$$
N_{1}=2 N_{0}-F_{0}-N_{-1}=2 N-F_{0} .
$$

Having obtained the rank of the next gauge group this way, one can repeat this process to recursively obtain the values for the consequitive gauge groups.

$$
\begin{aligned}
& N_{2}=2 N_{1}-F_{1}-N_{0}=3 n-2 F_{0}-F_{1} \\
& N_{3}=2 N_{2}-F_{2}-N_{1}=4 n-4 F_{0}-2 F_{1}-F_{2}
\end{aligned}
$$


A quiver defined in this way will automatically terminate itself by giving $N_{\text {last }}=0$, for the last value of the rank function. This can be seen from the consistency condition, which dictates that the values of the flavour groups are proportional to the second derivative of the rank function

$$
\begin{aligned}
F_{k}= & \left(N_{k}-N_{k-1}\right)-\left(N_{k+1}-N_{k}\right) \\
& \Delta N_{k}-\Delta N_{k+1}=\Delta^{2} N_{k}
\end{aligned}
$$

As the values of the flavour groups have to be greater than or equal to zero everywhere, the rank function has to be concave, and will go back to zero eventually.

By choosing $N$ an arbitrarily large number and using an arbitrarily large number $k$ of flavours this method automatically defines a quiver of arbitrary length and arbitrary large gauge and flavour groups for which the supergravity limit is valid.

\subsubsection{Parabolic Quiver}

As an example we will start here with a quiver where all the flavour groups are equal to some arbitrary integer number

$$
F_{k}=f
$$

And choose the rank for the first gauge group on the quiver to be equal to some other arbitrary number $N_{1}=n$. The ranks of the gauge groups will be

$$
N_{k}=n k-\sum_{j=1}^{k}(j-1) f
$$

If we set $N_{1}=n$ we find from the consistency condition eq.(6.17) that this will result in a rank function $R(z)=(2,2)$, and the quiver will terminate after 2 colour nodes as can be seen in figure 6.1. For a generic quiver of this form, with $n$ and $f$ arbitrary numbers, the rank function will return to zero for $N_{k}$ when $k=2 n / f+1$. We will first calculate the total number of branes and central charges associated with brane set-ups involving this quiver. We then show that in the limit $n / f \rightarrow \infty$ the rank function asymptotes to that of continuous parabola (show with the dotted grey line in figure 6.1).

The total number of $D_{d}$ and $D_{d+2}$ branes corresponding to this discrete quiver will be given by

$$
\begin{aligned}
N_{D_{d}} & =\sum_{k=1}^{2 n / f}\left(k n-\sum_{j=1}^{k}(j-1) f\right)=n\left(\frac{2 n^{2}}{3 f^{2}}+\frac{n}{f}+\frac{1}{3}\right) \\
N_{D_{d+2}} & =2 n
\end{aligned}
$$


VI. INTEGRABLE STRINGS ON $\mathrm{ADS}_{7} \times\left(S^{3}\right)_{\lambda}$

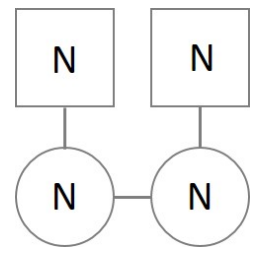

(a)

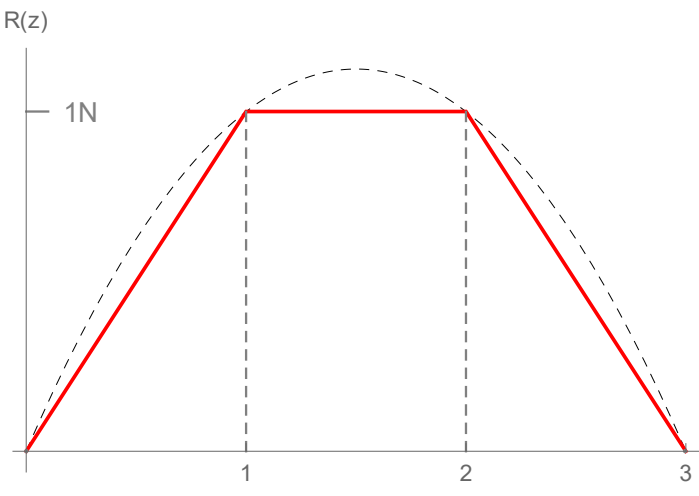

(b)

Figure 6.1: (left) the quiver, and (right) the rank function (in red) for $n=1$ and $f=1$.

Using this rank function in eq.(6.22) and the result from eq.(4.35) we can calculate the central charges of the corresponding $d=4$ and $d=6$ dimensional field theories as (with $P=2 n / f$ )

$$
c_{d=4}=\sum_{k=1}^{P} N_{k}^{2}=n^{2}\left(\frac{2 n^{2}}{3 f^{2}}+\frac{n}{f}+\frac{1}{3}\right)^{2}
$$

For the $6 d$ case we find

$$
\begin{aligned}
& b_{0}=\frac{-1}{\frac{2 n}{f}+1} \sum_{i=1}^{2 n}\left(\frac{2 n}{f}+1-i\right) N_{i} \quad=\frac{-n}{6 f^{2}}(f+n)(f+2 n) \\
& a_{k}=k b_{0}+\frac{N_{k}}{6}+\sum_{i=1}^{k}(k-i) N_{i} \quad=k b_{0}+\frac{k^{2}}{24}\left(4 k n-f(k-1)^{2}\right) \\
& b_{k}=b_{0}+\frac{N_{k}}{2}+\sum_{i=1}^{k-1} N_{i} \quad=b_{0}+\frac{k}{12}(6 k n-f(k-1)(2 k-1))
\end{aligned}
$$

giving us for the central charge

$$
c_{d=6}=-n^{2}\left(\frac{34 n^{5}}{315 f^{5}}+\frac{17 n^{4}}{45 f^{4}}+\frac{47 n^{3}}{90 f^{3}}+\frac{13 n^{2}}{36 f^{2}}+\frac{23 n}{180 f}+\frac{f}{2520 n}+\frac{7}{360}\right)
$$

The larger we make the ratio $n / f$, the closer the rank function of this quiver will approximate the function

$$
R(z)=\left(n+\frac{f}{2}(1-z)\right) z
$$




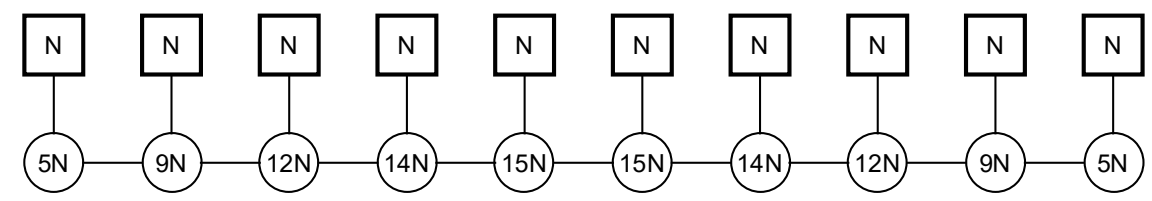

(a)

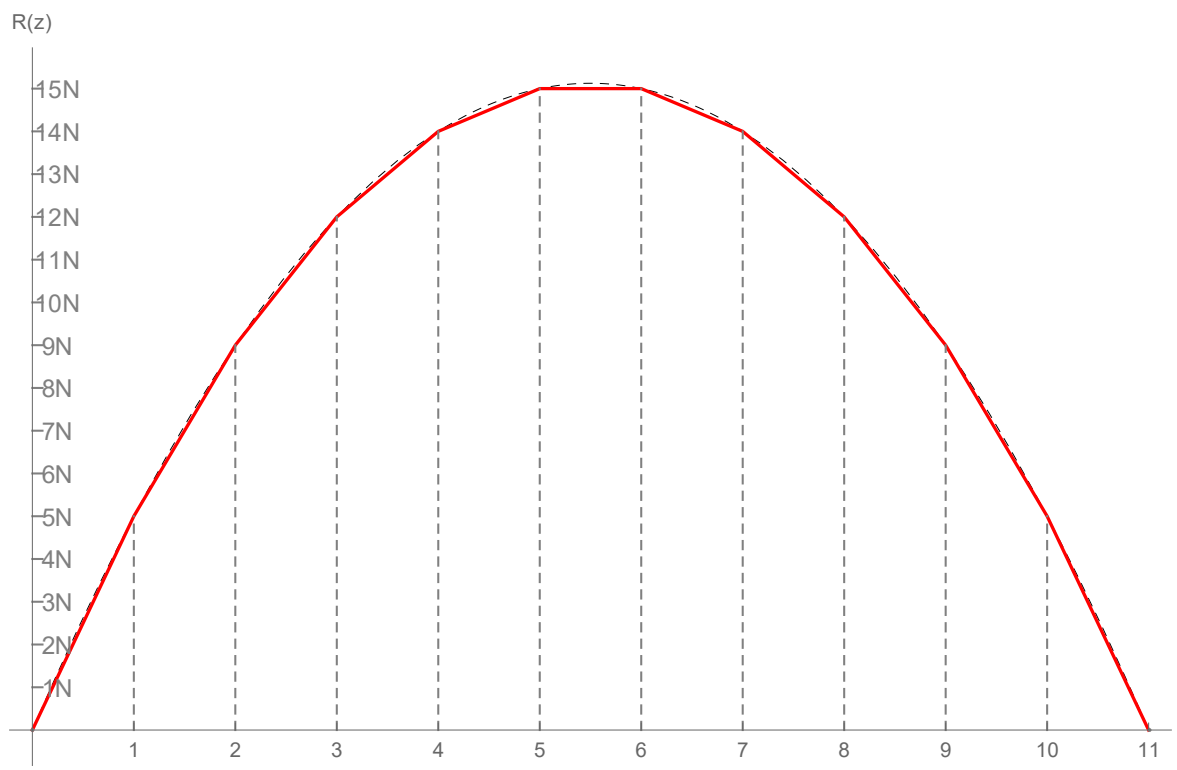

(b)

Figure 6.2: (a) the quiver, and (b) the rank function (in red) for $n=5$ and $f=1$.

See figure 6.2, where we illustrate this with an example for $n=5$ and $f=1$.

The simplest way to think about this intuitively is that the flavour groups give the second derivative of the rank function (see section 6.5.1). When the flavour groups are constant everywhere, the rank function thus needs to have a constant second derivative and will be parabolic. As we choose the value of $n / f$ larger we will approximate a parabola with more points, which becomes continuous in the limit where we take $n / f \rightarrow \infty$.

We will now calculate the number of branes and central charges from the continuous rank function and show they indeed match as we take the limit $n / f \rightarrow \infty$. The number of branes 
can simply be calculated as

$$
\begin{aligned}
N_{D_{p}} & =\int d z R(z)=n\left(\frac{2 n^{2}}{3 f^{2}}+\frac{n}{f}\right) \\
N_{D_{p+2}} & =-\int d z R^{\prime \prime}(z)=2 n
\end{aligned}
$$

Note this indeed agrees with the large $n / f$ limit of eq.(6.23) and (6.24).

The central charge for the $4 \mathrm{~d}$-Maldacena case can be calculated as

$$
c=\frac{2}{\pi^{4}} \int_{0}^{P+1} \lambda^{2}(z) d z=n^{2}\left(\frac{2 n^{2}}{3 f^{2}}+\frac{n}{f}\right)^{2}
$$

using that $\lambda(z)=R(z)$. Note this indeed corresponds with the large $n / f$ limit of eq.(6.25).

To calculate the central charge for the $6 \mathrm{~d}$ Cremonesi-Tomasiello case we need the function $\alpha(z)$ that is proportional to the second integral of the rank function. Demanding that $\alpha^{\prime}(z)=0$ for $z=\frac{1}{2}\left(\frac{2 n}{f}+1\right)$, and $\alpha(z)=0$ for $z=\left(\frac{2 n}{f}+1\right)$ we find

$$
\begin{gathered}
\alpha^{\prime}(z)=\frac{1}{12}(6 n+f(3-2 z)) z^{2}-\frac{(f+2 n)^{3}}{24 f^{2}} \\
\alpha(z)=\frac{z^{3}}{24}(2 f+4 n-f z)-\frac{(f+2 n)^{3}}{24 f^{2}} z
\end{gathered}
$$

which gives us for the central charge

$$
c_{d=6}=-n^{2}\left(\frac{34 n^{5}}{315 f^{5}}+\frac{17 n^{4}}{45 f^{4}}+\frac{17 n^{4}}{30 f^{3}}+\frac{17 n^{2}}{36 f^{2}}+\frac{17 n}{72 f}+\frac{17 f n}{1440}+\frac{17 f^{2}}{20160 n^{2}}+\frac{17}{240}\right)
$$

Note this result again corresponds with the large $n / f$ limit of eq.(6.26).

We thus find that in the limit that $n / f \rightarrow \infty$ the continuous rank function from eq.(6.30) indeed captures the correct number of D-branes and central charges.

\subsubsection{Sinusoidal Quiver}

We will now discuss a continuous rank function of the form

$$
R(z)=A \sin \omega z .
$$

This particular example is especially interesting as it was recently shown in [106] that the bosonic sector of the string is integrable on the $\mathrm{AdS}_{7}$ Cremonesi-Tomasiello geometry corresponding to this rank function. 
The Gaiotto-Maldacena $\mathrm{AdS}_{5}$ solution corresponding to this rank function was discussed in detail in [81] (section 4), its analytic continuation has been discussed in [40] (see eq.(2.44) of that paper).

For this sinusoidal quiver the rank function has the property $R(z)=-\omega^{-2} R^{\prime \prime}(z)$. This means all the flavour groups are proportional to the colour groups, as the number of flavour branes everywhere has to be proportional to the second derivative of the rank function. We can, therefore, approximate this continuous rank function by a discrete one, using as a defining condition that the number of flavours is everywhere proportional to the colour groups. ${ }^{1}$

$$
N_{k}=\epsilon F_{k}
$$

If we take this to be the defining condition for the quiver and set $N_{0}=F_{0}=0$ at the beginning of the interval, we can use the consistency condition in eq.(6.17) to recursively define the values of $N_{k}$ for a quiver or arbitrary length from the consistency condition

$$
\begin{aligned}
N_{k} & =2 N_{k-1}-F_{k-1}-N_{k-2} \\
& =\left(2-\frac{1}{\epsilon}\right) N_{k-1}-N_{k-2}
\end{aligned}
$$

This example proves to be a little more complicated than the parabolic quiver, and it is more difficult to give a closed expression for the different ranks $N_{k}$

$$
N_{k}=n k-\sum_{i=1}^{k-1} f_{k} \quad \text { where } \quad F_{k-1}=\frac{1}{\epsilon}\left(n(k-1)-\sum_{i=1}^{k-2} f_{k}\right)
$$

As the rank $N_{k}$ is given in terms of the previous ranks and flavours, and the number of flavours is now proportional to the rank - which in turn is given in terms of all the previous ranks and flavours - we find that $N_{k}$ is given as a recursive summation.

If we work this out and set $N_{1}=n$ we find first the terms look like

$$
\begin{aligned}
& N_{2}=2 n-\frac{n}{\epsilon} \\
& N_{3}=3 n-\frac{4 n}{\epsilon}+\frac{n}{\epsilon^{2}} \\
& N_{4}=4 n-\frac{10 n}{\epsilon}+\frac{6 n}{\epsilon^{2}}-\frac{n}{\epsilon^{3}} \\
& N_{5}=5 n-\frac{20 n}{\epsilon}+\frac{21 n}{\epsilon^{2}}-\frac{8 n}{\epsilon^{3}}+\frac{n}{\epsilon^{4}} \\
& N_{6}=6 n-\frac{35 n}{\epsilon}+\frac{56 n}{\epsilon^{2}}-\frac{36 n}{\epsilon^{3}}+\frac{10 n}{\epsilon^{4}}-\frac{n}{\epsilon^{5}}
\end{aligned}
$$

\footnotetext{
${ }^{1}$ Note that the shortest possible quiver of this type is the $N_{F}=2 N_{C}$ quiver, when we set $\epsilon=\frac{1}{2}$.
} 
As an example let us now obtain a quiver with only three colour groups that approximates a sinusoidal quiver. This means we want $N_{5}=0$, so we can solve for $\epsilon$

$$
5 n \epsilon^{4}-20 n \epsilon^{3}+21 n \epsilon^{2}-8 n \epsilon+n=0,
$$

which has the roots

$$
\epsilon_{ \pm}^{1}=\frac{1}{2}(3 \pm \sqrt{5}) \quad \text { and } \quad \epsilon_{ \pm}^{2}=\frac{1}{10}(5 \pm \sqrt{5}) .
$$

The different roots will now all correspond to quivers with rank functions that are piece-wise continuous approximations of sine functions (see figure 6.3), all vanishing at $z=N_{5}$. We can

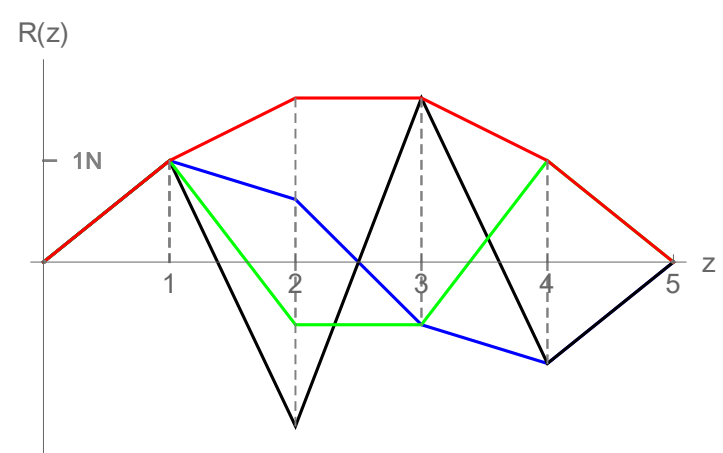

(a)

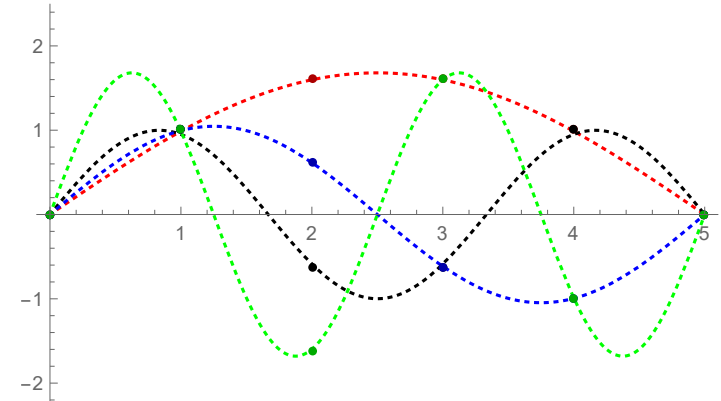

(b)

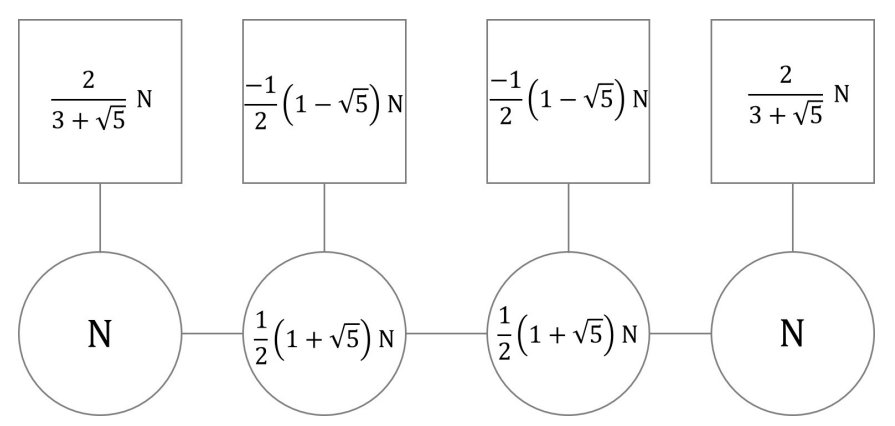

(c)

Figure 6.3: (a) Piece-wise continuous rank functions for the different values of $\epsilon$ : $\epsilon_{+}^{1}$ (red), $\epsilon_{-}^{1}$ (green), $\epsilon_{+}^{2}$ (black) and $\epsilon_{-}^{2}$ (blue). (b) Corresponding continuous rank functions that are approximated by these discrete ones. (c) Quiver diagram for $\epsilon_{+}^{1}$. Note that the values of all flavour groups are proportional to $2 /(3+\sqrt{5})$ times the corresponding colour groups.

generalise this example to generate piecewise continuous approximations to the sinusoidal rank function of any length, by requiring that the appropriate $N_{k}=0$. Eq.(6.40) will then give 
a $(k-1)$-th order polynomial in $\epsilon$, the different roots of which will correspond to piecewise continuous approximations to sinusoidal functions of different wavelengths all vanishing at $z=k$. The largest of these roots will always correspond to an approximation of a sine with period $2 k$.

As we take in eq.(6.38) the limit $\epsilon=\frac{N}{F} \rightarrow \infty$, the length of the largest sine wave becomes infinite. In this limit, the piecewise continuous rank functions will have the same numbers of colour and flavour branes associated with it as one would obtain from the continuous rank function, the way we illustrated earlier for the parabolic quiver.

\subsection{Smeared Branes}

In the previous section, we started by defining infinitely long quivers and argued they are described by continuous rank functions in their gravitational dual. We will now reason the other way around: we start by considering a supergravity solution with a continuous rank function, and we show this implies the flavour branes that backreact on the geometry are smeared. We then show the correct way to interpret the corresponding brane set-up is as an infinitely long quiver described in the previous section.

When we consider the supergravity solutions in eqs.(3.35) and (4.6) with a rank function $R(z)$, the second derivative $R^{\prime \prime}(z)$ indicates the positions of the flavour branes backreacting on the geometry.

When we consider a piecewise linear rank function $R(z) \in C^{(0)}$ (as in section 3.5.2), the positions of the flavour branes are given by delta functions $R^{\prime \prime}(z)=\sum_{i} \delta\left(z_{i}\right)$. When we consider a continuous rank function $R(z) \in C^{(\infty)}$, the second derivative will no longer be a series of delta functions, but will instead be continuous $R^{\prime \prime}(z)=\rho(z)$. This implies the flavour branes are no longer located at fixed points, but are instead smeared along the $z$-direction of the geometry. The geometry is now a solution of the equations of motion for the action

$$
S_{10 d \text { Type II }}+\int d z \rho(z) S_{B I W Z}
$$

which differs from the action in eq.(3.25) in that the $D_{p+2}$-flavour branes are not located at fixed points given by delta functions, but instead are smeared with a certain density $\rho(z)$. The backreaction of smeared flavour branes was first introduced in [133], and further studied in a $10 \mathrm{~d}$ context in $[134,150]$

Since the $D_{p+2}$ flavour-branes act as sources for the $F_{8-(p+2)}$ RR-flux, the Bianchi identities are violated at the points where these flavour branes are located. In addition, the curvature will typically diverge at the points where these flavour branes are located.

When we consider a continuous rank function, the delta functions are 'smeared' and the resulting geometries no longer has singular points (as can be verified by inspecting the dilaton 


\section{INTEGRABLE STRINGS ON $\operatorname{ADS}_{7} \times\left(S^{3}\right)_{\lambda}$}

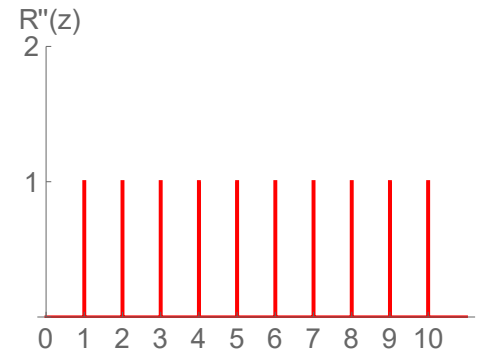

(a)

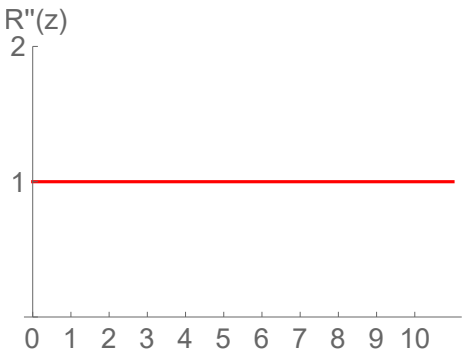

(b)

Figure 6.4: The positions of the flavour branes for (a) the discrete and (b) the continuous parabolic rank function of figure 6.2. The continuous rank function implies a continuously smeared distribution of flavour branes.

and curvature). This smearing then causes the Bianchi identities to be violated everywhere along the $z$-direction ${ }^{2}$

$$
d F_{8-(p+2)}=\rho(z) .
$$

Unlike the examples studied in [150], we cannot just add a smeared number of flavour branes to the half-BPS backgrounds we consider in this paper. Since the numbers of colour and flavour branes are not independent of each other, but are both related to the rank function $R(z)$ though the consistency condition, eq.(3.24), smearing the flavour branes then implies the number of colour branes has to become infinite as well. This can also be seen from the Page charges for the total number of flavour and colour branes in eq.(4.33), which are both related to the rank function as

$$
\begin{aligned}
N_{D_{p+1}} & =\int_{0}^{P+1} d z R^{\prime \prime}(z), \\
N_{D_{p}} & =\int_{0}^{P+1} d z R(z) .
\end{aligned}
$$

Since the colour branes have to end on NS5-branes in the corresponding Hanany-Witten set-up, we cannot have let the number of colour branes go to infinity without simultaneously letting the number of NS5-branes go to infinity. This ensures that all the colour branes end on NS5-branes.

When we consider a finite continuous rank function $R(z)$ for $z \in[0,1]$, increasing the number of NS5-branes is done by the scaling $z \rightarrow \omega z$ we discussed earlier in section 3.5.2. This means we have to rescale the continuous rank function to become infinitely long. We thus find that we can interpret any (concave) continuous rank function after 'stretching' it as accurately

\footnotetext{
${ }^{2}$ See Appendix A of [150] for an interesting relation between the violation of the Bianchi identities, supersymmetry, and calibrated geometry.
} 
approximated by a piecewise continuous quiver of infinitely many points (for which there is a well-defined brane set-up), as we showed in the previous section.

We can think of these continuous rank functions with smeared branes as 'macroscopic' descriptions of the infinite quivers defined in the previous section, where the infinite numbers of branes become continuous distributions.

\subsubsection{Orthogonal Bases of Quivers}

Since the rank functions can be decomposed into various orthogonal bases of functions, this naturally brings about the question if such bases have some interpretation in the dual SCFTs.

The differential equations for the functions that specify the half-BPS AdS geometries are linear (see eqs. (4.8) and (3.36) for the $\mathrm{AdS}_{5}$ and $\mathrm{AdS}_{7}$ cases respectively). We can therefore obtain any solution as a superposition of modes. Since we have shown in the previous sections how to interpret continuous rank functions as corresponding to infinite quivers, we now try to find a field theory interpretation for this basis of functions

$$
\alpha_{\mathrm{AdS}_{7}}(z)=-\sum_{n=1}^{\infty} A_{n} \sin \left(\omega_{n} z\right), \quad V_{\mathrm{AdS}_{5}}(\sigma, z)=-\sum_{n=1}^{\infty} c_{n} K_{0}\left(\omega_{n} \sigma\right) \sin \left(\omega_{n} z\right) .
$$

The rank functions corresponding to these solutions will be sinusoidal quiver with the ranks of its colour groups proportional to its flavour groups $N_{n}=\epsilon F_{n}$ that we introduced in section 6.5.3. It would be very interesting to better understand if this linearity of the differential equations (4.8) and (3.36) reflects some deeper property of the SCFTs.

As an example, we cite the result that the central charge in eq.(4.34) of an arbitrary 6d quiver SCFT, is directly given as a superposition of the central charges of these modes $[136,135] .{ }^{3}$

$$
c_{6 d}=\frac{-2^{8}}{3^{8} \times 16 \times G_{N}} \int_{0}^{P+1} d z \alpha^{\prime \prime}(z) \alpha(z), \quad c_{4 d}=\frac{2}{\pi^{4}} \int_{0}^{P+1} d z \lambda^{2}(z) .
$$

Note that since the different modes are orthogonal, and $\alpha^{\prime \prime}(z) \sim \alpha(z)$, the above computation becomes the sum over the contributions to the central charge from each mode. It would be very interesting to better understand this relation from the SCFT point of view.

In general, we expect other observables of the SCFTs to not linearly depend on the modes in eq.(6.48). This can be seen as the $\mathrm{AdS}_{5}$ and $\mathrm{AdS}_{7}$ geometries in eqs.(3.35, 4.6) depend on various combinations of the functions $\alpha(z)$ and $V(z, \sigma)$ and their derivatives. Even though the differential equations for $\alpha(z)$ and $V(z, \sigma)$ are linear, this linearity will not be reflected in the geometry, or the string dynamics on these spaces. As a result, observables like Wilson lines (that depend on the string dynamics) or the entanglement entropy of certain regions (which depends on the embedding of minimal surfaces) will not be linear in these modes.

\footnotetext{
${ }^{3}$ See section 2.3 of [136], section 5 of [106], see also [135].
} 


\section{INTEGRABLE STRINGS ON $\operatorname{ADS}_{7} \times\left(S^{3}\right)_{\lambda}$}

\section{Polynomial Bases}

It would be interesting to see if more quantities could be calculated directly from the rank function, and if other bases of functions - orthogonal under different inner products - might lend themselves to particular calculations.

Let us briefly remark that when constructing a basis of functions, we do not have to require the rank functions for these basis functions to satisfy the consistency condition. It is sufficient that the final superposition of modes satisfy this relation.

$$
F_{n}=2 N_{n}-N_{n+1}-N_{n-1}
$$

One could use the tools of section 6.5.2 to construct in an analogous fashion a basis of orthogonal polynomial rank functions.

\subsection{Field Theory Interpretation of the $\mathbf{A d S}_{7} \times\left(S^{3}\right)_{\lambda}$ Background}

We have shown how to make sense of continuous rank functions, as describing infinitely long quivers for which the supergravity description is valid. These infinite quivers with continuous rank functions $R(z)$ can be defined by fixing their flavour groups and fixing the colours by requiring that consistency conditions are satisfied.

These continuous rank functions generalise the solutions one normally considers for the supergravity solutions that describe the near-horizon limit of these brane set-ups. Since the supergravity requires quivers to be very large it might in fact be more convenient to think of all (concave) rank functions as describing valid supergravity solutions that can be interpreted as infinitely long quivers where the number of NS5, $\mathrm{D}_{p}$ and $\mathrm{D}_{p+2}$-branes have all become infinite. As we have seen in the examples for the parabolic and sinusoidal quivers (in sections 6.5.2 and 6.5.3 respectively), we have to take the limit $N / F \rightarrow \infty$, keeping $F$ fixed, for the rank function to become continuous. This corresponds to the large $N$ or 't Hooft limit in the dual SCFT.

Performing calculations for a generic long quiver of the kind obtained after the scaling procedure in section 3.5.2 can be quite difficult. But to study the SCFT corresponding to the sinusoidal quiver we can set all of the $N_{n}=\epsilon F_{n}$ and take the 't Hooft limit. Similarly one could use other defining conditions for the flavour groups to study different continuous rank functions. It would be interesting to see if certain calculations for quiver CFTs in question become more accessible in these cases.

For the 6d SCFT corresponding to the $\mathrm{AdS}_{7}$ background in eqs.(6.3)-(6.5) we however do not have a Lagrangian description, as we explained in more detail in section 4.1.1. Instead, we will define the CFT in terms of its $\mathrm{AdS}_{7}$ dual, discuss some of its properties, and calculate some of its observables. 


\subsubsection{Large Chern-Simons Level}

For the supergravity approximation to be valid, we need the string tension $T$ to be small compared to the curvature $R$ of the space. In the case of $\mathrm{AdS}_{5} \times \mathrm{S}^{5}$ it is well known how both these parameters are related to the Yang-Mills coupling $g_{Y M}$ and large $N$ expansion in the dual $\mathcal{N}=4$ SYM theory and the 't Hooft coupling.

$$
g_{Y M}^{2} N=\lambda=2 \pi \frac{R^{2}}{T}
$$

A natural question to ask is what parameter in the $6 \mathrm{~d}(1,0)$ SCFT plays a similar role to $g_{Y M}$, and could be related to the string tension. As we have seen in eq.(4.2) the coupling for the Yang-Mills field $A_{\mu}$ is fixed at infinity at the conformal fixed point, and can therefore not play this role. We should thus be able to identify some other parameter in the 6d SCFT that scales with the radius of our background in the holographic description.

A hint here comes from the parameter $k$ in the integrable background in eq.(6.12). On the string worldsheet this parameter comes from the Chern-Simons level of the WZW-model on $S U(2)$, and has to take on integer values.

Since these 6d SCFTs come with a Chern-Simons term with integer level, it then seems natural to identify this parameter $k$ with the level of the Chern-Simons term in the $6 \mathrm{~d} \mathcal{N}=(1,0)$ SCFT. This would then be analogous to the case of the ABJM model [8], where the integrable limit also corresponds to the Chern-Simons level $k \rightarrow \infty$.

\subsubsection{Central Charge}

We consider the solution derived from $\alpha(z)=A \sin (\omega z)$ and choose $\omega=\frac{n \pi}{N_{5}}$ which makes the coordinate range in $0 \leq z \leq \frac{N_{5}}{n}$. We can calculate the number of D6 and D8-branes in this background. Using eqs.(4.16),(4.19) we find,

$$
\begin{aligned}
& N_{D 6}=-\frac{1}{81 \pi^{2}} \int_{0}^{N_{5}} \alpha^{\prime \prime}(z) d z=\frac{2 A}{81 \pi N_{5}}, \\
& N_{D 8}=\frac{1}{81 \pi^{2}}\left[\alpha^{\prime \prime \prime}(0)-\alpha^{\prime \prime \prime}\left(N_{5}\right)\right]=-\frac{2 A \pi}{81 N_{5}^{3}} .
\end{aligned}
$$

In absolute value, these expressions imply relations among the quantities,

$$
A=\frac{81 \pi}{2} N_{5} N_{D 6}, \quad A=\frac{81}{2 \pi} N_{5}^{3} N_{D 8}, \quad \pi^{2} N_{D 6}=N_{D 8} N_{5}^{2}
$$




\section{INTEGRABLE STRINGS ON $\operatorname{ADS}_{7} \times\left(S^{3}\right)_{\lambda}$}

We can use the expressions for the linking numbers - eqs.(4.22),(4.23) and the expression for $A$ in terms of the number of flavour D8-branes,

$$
\sum_{i=1}^{N_{5}} K_{i}=\frac{1}{81 \pi^{2}} \alpha^{\prime \prime \prime}\left(N_{5}\right) N_{5}=\frac{A \pi}{81 N_{5}^{2}}=-\frac{N_{5} N_{D 8}}{2} .
$$

In the paper [136], the authors found an expression for the central charge of the conformal quiver - see eq.(2.14) in [136]. This coincides with the holographic central charge $a$ found in [74], derived by field theoretical means. Let us apply this expression for the case at hand. For $\alpha(z)=A \sin (\omega z)$, we find

$$
c=-\frac{2^{8}}{3^{8} \times 16 \times G_{N}} \int_{0}^{z_{f}} \alpha(z) \alpha^{\prime \prime}(z) d z=\frac{8}{3^{8} \times G_{N}} A^{2} \omega^{2} N_{5}=\frac{N_{D 6}^{2} N_{5}}{4 \pi^{2}} .
$$

We have used the expression for $A$ in terms of the number of colour D6-branes and that in our conventions $G_{N}=8 \pi^{6}$.

\subsubsection{Entanglement Entropy}

We calculate the entanglement entropy between two different regions $A$ and $B$ for the ground state $|0\rangle$ of the $6 \mathrm{~d}$ SCFT.

$$
A=\mathbb{R}^{5} \times I_{L}, \quad B=\mathbb{R}^{g} \times\left(\mathbb{R}-I_{L}\right) .
$$

where $I_{L}$ is an interval of length $L$. The entanglement entropy is defined as the entropy seen by an observer in region $\mathrm{A}$, who has to trace out the degrees of freedom in region $\mathrm{B}$ from the total density matrix $\rho_{0}=|0\rangle\langle 0|$

$$
S_{E E}=-\operatorname{Tr}_{A} \rho_{A} \ln \rho_{A}, \quad \text { where } \quad \rho_{a}=\operatorname{Tr}_{B} \rho_{0} .
$$

In terms of the holographic description as an $\mathrm{AdS}_{7}$ supergravity solution, the entanglement entropy can then be calculated directly from the Ryu-Takayanagi $[59,60,166]$ minimal surface that on the AdS boundary coincides with the boundary of the region A. In this case the boundary of the region $A$ looks like two copies of $\mathbb{R}^{5}$ separated by a distance $L$. The area of this surface is proportional to the entangelment entropy between the regions $A$ and $B$. A regularisation is needed analogously to what happens when computing Wilson loops, see [137] for the details. Since we separate the SCFT into two regions along one direction (and we ignore time) the resulting minimal surface is eight dimensional. The entanglement entropy is then given by

$$
S_{E E}=\frac{1}{4 G_{N}} \int d^{8} \sigma e^{-2 \phi} \sqrt{\operatorname{det} g_{8, i n d}}
$$


where $G_{N}=8 \pi^{6} g_{s}^{2} \alpha^{4}=8 \pi^{6}$ and $g_{8, \text { ind }}$ is the induced metric on the eight dimensional surface. In our treatment here we follow the notation presented in [137]

In particular for the backgrounds of the form in eq.(4.6). The eight surface is parametrised by the coordinates

$$
\Sigma_{8}=\left[x_{1}, x_{2}, x_{3}, x_{4}, x_{5}, z, \chi, \xi\right], \quad R=R\left(x_{1}\right) .
$$

Using Poincaré coordinates for the $\mathrm{AdS}_{7}$ space, the induced metric of the eight-surface is

$$
d s_{8, i n d}^{2}=f_{1}\left[R^{2} d \vec{x}_{4}^{2}+d x_{1}^{2}\left(R^{2}+\frac{R^{2}}{R^{2}}\right)\right]+f_{2} d z^{2}+f_{3}\left(d \chi^{2}+\sin ^{2} \chi d \xi^{2}\right) .
$$

The entanglement entropy is then given by

$$
\begin{aligned}
& S_{E E}=\frac{128 V_{4}}{6561 G_{N}}\left(\int_{0}^{z_{f}} \alpha^{\prime \prime}(z) \alpha(z) d z\right) \int d x_{1} R^{5} \sqrt{1+\frac{R^{\prime 2}}{R^{4}}}, \\
& V_{4}=\int d x_{2} d x_{3} d x_{4} d x_{5} .
\end{aligned}
$$

We are interested in the $L$ dependence of the entanglement entropy. When the gravitational background is dual to a confining gauge theory, we typically find there are two kinds of minimal surfaces possible: For large values of $L$ there are typically two disconnected surfaces, so that $S_{E E}$ does not depends on $L$. As we go to smaller values of $L<L_{\text {crit }}$ a smaller minimal surface becomes possible where the two separate surfaces are now connected by a strip whose width depends on $L$. One can think of this as signalling a phase transition that is similar to a confinement-deconfinement one.

Following the formalism of the works [137], we find the regularised version of the entanglement entropy, $S_{E E}^{r e g}$ and the separation between the regions $L$ to be,

$$
\begin{aligned}
& S_{E E}^{r e g}=\frac{V_{4}}{2 G_{N}}\left[\int_{1}^{\infty} d y\left(\frac{y^{8}}{\sqrt{y^{10}-1}}-y^{3}\right)\right] \mathcal{N} R_{0}^{4}=\mu_{1} \mathcal{N} R_{0}^{4}, \\
& L=\left[2 \int_{1}^{\infty} \frac{d y}{\sqrt{y^{4}\left(y^{10}-1\right)}}\right] \frac{1}{R_{0}}=\frac{\mu_{2}}{R_{0}}
\end{aligned}
$$

Giving us

$$
S_{E E}^{r e g}=\mathcal{N}\left(\frac{\mu_{1} \mu_{2}^{4}}{L^{4}}\right), \quad \mathcal{N}=-\frac{512}{6561} \int_{0}^{z_{f}} \alpha(z) \alpha^{\prime \prime}(z) d z
$$

The factors $\mu_{1} \mu_{2}^{4}$ are common to all six-dimensional conformal field theories. The power $L^{-4}$ is the only possible one given conformality and the dimension of the CFT. All the information about the particular CFT in consideration is in the factor $\mathcal{N} \sim \int \alpha \alpha^{\prime \prime}$. Notice that this factor also appears when computing the central charge of the CFT, see [136]. This is not a surprise as both quantities measure the number of degrees of freedom. 


\section{INTEGRABLE STRINGS ON $\operatorname{ADS}_{7} \times\left(S^{3}\right)_{\lambda}$}

Using the expression in eq.(6.60), we find that for the SCFT corresponding to $\alpha(z)=$ $A \sin (\omega z)$

$$
S_{E E}^{r e g}=\left(\frac{\mu_{1} \mu_{2}^{4}}{L^{4}}\right) \times 64 \pi^{4} N_{D 6}^{2} N_{5},
$$

Notice that $L\left(R_{0}\right)$ is a monotonically decreasing function that diverges at the origin and goes to zero at the boundary. For $S_{E} E(L)$ we find two possible phases: the first (connected phase) grows monotonically with $L$, while the second (the disconnected phase) is constant. The second disconnected phase thus always has a larger entanglement entropy than the connected phase, and is never favoured. Therefore there is no phase transition, as is indeed appropriate for a conformal field theory.

An interesting observation is that these expressions for the linking numbers, central charge and entanglement entropy in eqs.(6.55),(6.56),(6.61) have the same scaling with $N_{D 6}$ and $N_{5}$ as a four-dimensional $\mathcal{N}=2$ quiver that starts with a flavour group of rank $N_{D 6}$, continues with $N_{5}-1$ colour groups of rank $N_{D 6}$ and closes with a flavour group of rank $N_{D 6}$. See around eq.(3.16) of [135].

\subsubsection{Wilson Loops}

Another interesting observable in a CFT is the Wilson loop [199]. These are operators of the form

$$
W(\mathcal{C})=\frac{1}{N} \operatorname{Tr} \mathcal{P} \exp \left[i \oint_{\mathcal{C}} d s A_{\mu} \dot{x}^{\mu}\right]
$$

Here $\mathcal{C}$ is a contour with parametrisation $x^{\mu}(s)$, and $\mathcal{P}$ denotes the path ordering of the fields in terms of $s$.

By construction the Wilson loop $W(\mathcal{C})$ is a gauge invariant non-local operator, and is equivalent to the phase acquired by a quark that transforms under the fundamental representation of the gauge group and moves along the contour $\mathcal{C}$. From a thin rectangular Wilson loop with sides $R$ and $T$, and $T \gg R$, we can find the potential energy of a static quark-antiquark pair

$$
V(R)=-\lim _{T \rightarrow \infty} \frac{1}{T} \ln \langle W(\mathcal{C}(R, T))\rangle
$$

In order to obtain massive (static) quark for $\mathcal{N}=4 \mathrm{SYM}$, Maldacena [200] considered starting from $S U(N+1) \mathcal{N}=4 \mathrm{SYM}$ and breaking this to $S U(N) \times U(1)$, so the W-bosons acquire a mass and now transform under the fundamental representation of $S U(N)$. The scalars fields of the original $S U(N+1) \mathcal{N}=4 \mathrm{SYM}$ then break into the massless scalars of the remaining $S U(N)$ theory, and additional fields that obtain a mass and transform in the (anti-)fundamental 
representation of $S U(N)$. The Wilson loop operator for the massive W-bosons is then of the from

$$
W(\mathcal{C})=\frac{1}{N} \operatorname{Tr} \mathcal{P} \exp \left[i \oint_{\mathcal{C}} d s A_{\mu} \dot{x}^{\mu}+|\dot{x}| \Phi^{i} n_{i}\right]
$$

In terms of the holographic AdS description, Maldacena then proposed [200] that the expectation value of the Wilson loop is equal to the partition function of a string that ends on $\mathcal{C}$ at the AdS boundary

$$
W(\mathcal{C})\rangle=Z_{\text {string }}
$$

where the leading term will be given by the classical string configuration.

Here we will follow this approach and study similar Wilson loops in the six-dimensional $\mathcal{N}=(1,0)$ theory by considering static fundamental string on a generic $\mathrm{AdS}_{7}$ background of the form in eq.(4.6), parametrised by

$$
t=\tau, \quad x=\sigma, \quad R=R(\sigma), \quad z=z(\sigma)
$$

We use Poincaré coordinates for the $\mathrm{AdS}_{7}$ space, parametrised by $(t, \vec{x}, R)$. The Nambu-Goto action of the fundamental string on a generic background is,

$$
S_{N G}=\frac{1}{2 \pi} \int d \tau d \sigma \sqrt{f_{1}^{2} R^{4}+f_{1}^{2} R^{\prime 2}+f_{1} f_{2} R^{2} z^{\prime 2}}=\frac{T}{2 \pi} \int d \sigma \sqrt{f_{1}^{2} R^{4}+f_{1}^{2} R^{\prime 2}+f_{1} f_{2} R^{2} z^{\prime 2}} .
$$

This action does not depend explicitly on the 'time variable' $\sigma$ and this implies the conserved 'Hamiltonian',

$$
\frac{f_{1}^{2} R^{4}}{\sqrt{f_{1}^{2} R^{4}+f_{1}^{2} R^{\prime 2}+f_{1} f_{2} R^{2} z^{\prime 2}}}=C .
$$

At this point, it is interesting to analyse three situations:

- The situation for which the coordinate $z(\sigma)$ is constant. In this case, we are back to the usual Wilson loop calculation in strongly coupled CFTs [200], that gives $E_{Q Q} \sim \frac{\sqrt{\lambda}}{L_{Q Q}}$

- The situation in which $R(\sigma)=R_{0}$ is constant. In this case we find the Nambu-Goto action,

$$
S_{N G}=\frac{T}{2 \pi} \int d \sigma \sqrt{f_{1}^{2}(z) R_{0}^{4}+f_{1}(z) f_{2}(z) R_{0}^{2} z^{\prime 2}} .
$$

That leads to more a conventional minimisation problem, equivalent to the calculation of the 'usual' rectangular Wilson loop in a background of the form $d s^{2} \sim R_{0}^{2} f_{1}(z)\left[d x_{1, p}^{2}\right]+$ $f_{2}(z) d z^{2}$. Using eq.(4.7) we find that $f_{1}(z) f_{2}(z)=16 \pi^{2}$. The main difference with the situations calculated previously in the bibliography is that the $z$-coordinate is bounded. 
VI. INTEGRABLE STRINGS ON $\mathrm{ADS}_{7} \times\left(S^{3}\right)_{\lambda}$

- More interesting than the general study presented above is to consider the action in eq.(6.67) for the case of our special background in eq.(6.3), for which $f_{1}(z)=\frac{8 \sqrt{2} \pi}{\omega}$ and $f_{2}(z)=\sqrt{2} \pi \omega$. Using these values, the action in eq.(6.67) reads,

$$
S_{N G}=\frac{\sqrt{32} T}{\omega} \int d \sigma \sqrt{R^{4}(\sigma)+R^{\prime 2}(\sigma)+\frac{\omega^{2}}{8} R^{2}(\sigma) z^{\prime}(\sigma)^{2}} .
$$

We can redefine the variable $\tilde{z}=\frac{\omega z}{\sqrt{8}}$ and from the action in eq.(6.70) we find two conserved quantities,

$$
\frac{R^{4}}{\sqrt{R^{4}+R^{\prime 2}+R^{2} \tilde{z}^{\prime 2}}}=E, \quad \frac{R^{2} \tilde{z}^{\prime}}{\sqrt{R^{4}+R^{\prime 2}+R^{2} \tilde{z}^{\prime 2}}}=J, \quad R^{4} \frac{J^{2}}{E^{2}}=\tilde{z}^{\prime 2} .
$$

Following the usual procedure to write the separation of the external quarks in the $x$-direction $L_{Q Q, x}$ and in the $\tilde{z}$-direction $L_{Q Q, \tilde{z}}$,

$$
\begin{aligned}
& L_{Q Q, x}=\frac{E}{R_{0}^{3}} \int_{1}^{\infty} d y \frac{1}{y^{2} \sqrt{y^{4}-\frac{J^{2}}{R_{0}^{2}} y^{2}-\frac{E^{2}}{R_{0}^{4}}}}, \\
& L_{Q Q, \tilde{z}}=\frac{J}{R_{0}} \int_{1}^{\infty} d y \frac{1}{\sqrt{y^{4}-\frac{J^{2}}{R_{0}^{2}} y^{2}-\frac{E^{2}}{R_{0}^{4}}}} .
\end{aligned}
$$

The energy of the quark-antiquark pair $E_{Q Q}$ is after regularisation,

$$
E_{Q Q}=\frac{\sqrt{32} R_{0}}{\omega}\left[\int_{1}^{\infty} d y\left(\frac{y^{2}}{\sqrt{y^{4}-\frac{J^{2}}{R_{0}^{2}} y^{2}-\frac{E^{2}}{R_{0}^{4}}}}-1\right)-1\right]
$$

After redefining $\tilde{J} R_{0}=J$ and $\tilde{E} R_{0}^{2}=E$, we observe that these expressions in eqs.(6.72)-(6.73) are the same as those obtained by Maldacena in [200] when considering quarks that are charged under the R-symmetry. In our background the $z$-coordinate is not isometric, but the fundamental string in the configuration of eq.(6.66) does see it as part of the R-symmetry.

We conclude this chapter hoping to have given the reader a flavour of the many things that can be holographically computed with the $\mathrm{AdS}_{7} \times\left(S^{3}\right)_{\lambda}$ background. 
VII. T-DUALITY AS A SOLUTION GENERATING TECHNIQUE

\section{Chapter 7}

\section{T-Duality as a Solution Generating Technique}

In this chapter we will focus on the power of integrable deformations as tools to obtain new supergravity solutions.

In the previous chapter we saw that the $\operatorname{AdS}_{7} \times\left(\mathrm{S}^{3}\right)_{\lambda}$ background (on which we showed bosonic sector of the string is integrable) can be thought us as a lambda-deformation of $\mathrm{AdS}_{7} \times \mathrm{S}^{3}$ (which is not a supergravity background). In this chapter we will focuss on (nonAbelian) T-duality as it maps one supergravity background to another and preserves integrability. To illustrate the power of non-Abelian T-duality as solution generating technique we will construct a new solution of type IIB supergravity with eight supercharges by applying a non-Abelian T-duality to the $A d S_{5} \times S^{5}$ solution, along both the $S U(2)$ isometries in the internal and Lorentz symmetries.

The study of quantised charges and D-brane embeddings on the resulting background suggests a configuration of D1 and D3-branes that polarise into concentric, spherical, D3 and D5-branes due to the Myers dielectric effect. The low energy fluctuations are described by a IIB matrix model with fuzzy sphere vacua characterised by partitions of the number of D1 and D3-branes. The content of this chapter is largely based on the work published in [82].

Finding solutions to the supergravity equations can in general be quite complicated. Well known solutions as $\mathrm{AdS}_{5} \times S^{5}$ can often be found relatively easy as they have a very large number of (super)isometries. The fewer isometries a solution has, the more difficult it is to find solutions.

Since non-Abelian T-duality (NATD) maps us from one supergravity solution to another, and breaks the symmetries of the original space on which one performs the duality, it can be used as a 'solution generating technique' that allows us to obtain supergravity solutions with fewer isometries. Because NATD preserves integrability, this has the additional benefit that if 
we start from a background on which the superstring is integrable, this will still be the case on the resulting less symmetric dual background.

In this chapter we will show several examples of NATD as a solution generating technique applied to $\mathrm{AdS}_{5} \times S^{5}$, where we apply a NATD on either an $S^{3} \cong S U(2)$ subspace in the $\mathrm{AdS}_{5}$ or $S^{5}$ subspace, and on both of them. We give an overview of the supergravity solutions we will discuss here in figure 7.1 .

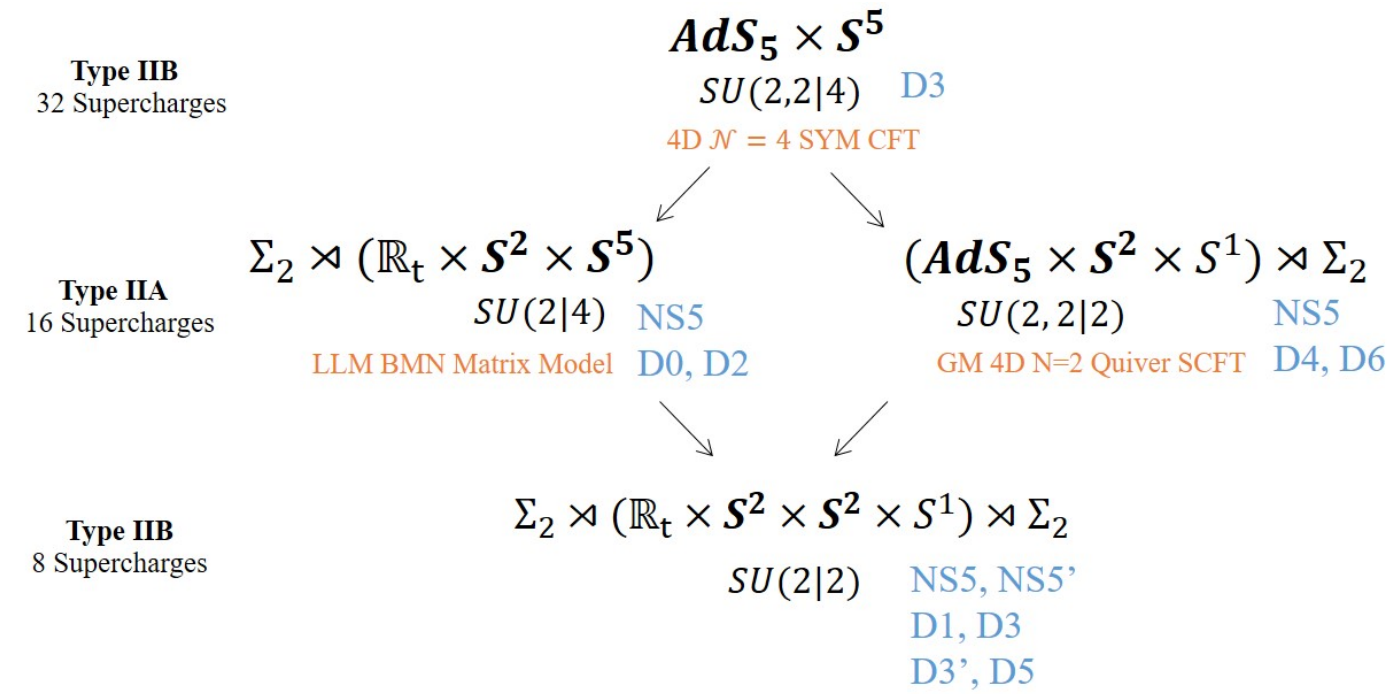

Figure 7.1: Schematic overview of the $\mathrm{AdS}_{5} \times \mathrm{S}^{5}$ solution and the geometries that are obtained by acting with a NATD on either of the $S U(2)$ 's in the $\mathrm{AdS}_{5}$ or $\mathrm{S}^{5}$ subspaces, or both. Below each background we write the largest superisometry group this background still possesses. In blue we write the types of branes that source the Ramond-Ramond and $H_{3}$ fluxes that are present on the resulting backgrounds. The low energy fluctuations of these branes are described by quantum field theories that are written here in orange.

\subsection{1 $\quad \mathrm{AdS}_{5} \times \mathrm{S}^{5}$ Solution}

In this section we start by giving a brief overview of the supergravity solutions that we will refer back to throughout the rest of this chapter. We refer the reader to [197, 37] for a more detailed explanation of their properties. All of the solutions that we will discuss here are obtained by applying T-duaities to the well-known type IIB $A d S_{5} \times S^{5}$ solution. The metric of this solution is

$$
d s^{2}=L^{2}\left(d s_{A d S_{5}}^{2}+d \Omega_{5}^{2}\right),
$$


where, in global coordinates, the line elements are

$$
\begin{aligned}
d s_{A d S_{5}}^{2} & =-\cosh ^{2} r d t^{2}+d r^{2}+\frac{\sinh ^{2} r}{4}\left(\omega_{1}^{2}+\omega_{2}^{2}+\omega_{3}^{2}\right), \\
d \Omega_{5}^{2} & =d \alpha^{2}+\sin ^{2} \alpha d \beta^{2}+\frac{\cos ^{2} \alpha}{4}\left(\sigma_{1}^{2}+\sigma_{2}^{2}+\sigma_{3}^{2}\right) .
\end{aligned}
$$

Here $\omega_{i}$ and $\sigma_{i}$ are two sets of left invariant Maurer-Cartan forms parametrising the $S U(2)$ isometries inside the $A d S_{5}$ and $S^{5}$ subspaces respectively. They are explicitly given by

$$
\begin{array}{ll}
\omega_{1}=\cos \psi \sin \theta_{1} d \phi_{1}-\sin \psi_{1} d \theta_{1}, & \sigma_{1}=\cos \psi \sin \theta d \phi-\sin \psi d \theta, \\
\omega_{2}=\sin \psi_{1} \sin \theta_{1} d \phi_{1}-\cos \psi_{1} d \theta_{1}, & \sigma_{2}=\sin \psi \sin \theta d \phi-\cos \psi d \theta, \\
\omega_{3}=d \psi_{1}+\cos \theta_{1} d \phi_{1}, & \sigma_{3}=d \psi+\cos \theta d \phi,
\end{array}
$$

where $\alpha \in\left[0, \frac{\pi}{2}\right], \beta \in[0,2 \pi], \phi, \phi_{1} \in[0,2 \pi], \theta, \theta_{1} \in[0, \pi]$ and $\psi, \psi_{1} \in[0,4 \pi]$. This solution is supported by a self-dual RR five-form field strength,

$$
F_{5}=\frac{4}{L}\left(\operatorname{Vol}\left(A d S_{5}\right)-\operatorname{Vol}\left(\Omega_{5}\right)\right)
$$

with $N_{D_{3}}$ units of flux ${ }^{1}$

$$
N_{D_{3}}=\frac{1}{2 \kappa_{10}^{2} T_{D_{3}}} \int_{S^{5}} F_{5}, \quad L^{4}=\frac{\pi}{4} g_{s}^{2} N_{D_{3}} \alpha^{2}
$$

This space has an $S U(2,2 \mid 4)$ superisometry group with 32 fermionic generators. The bosonic subgroup of the isometry group is $S U(2,2) \times S U(4) \cong S O(4,2) \times S O(6)$.

\subsubsection{NATD inside $S^{5}$ (The Sfetsos-Thompson Solution)}

In [190] a NATD was first applied along one of the $S U(2) \subset S O(6)$ isometries of the $A d S_{5} \times S^{5}$ solution. This will be the background we will introduce in this section.

To obtain the resulting supergravity solution, one can start from the $\operatorname{AdS}_{5} \times \mathrm{S}^{5}$ metric as written in eqs.(7.1) and (7.2) and apply a NATD to one of the one of the $S U(2) \subset S O(6)$ symmetry groups that are parametrised by the $\sigma_{i}$ 's in eq. (7.4). In order to do this one can follow the detailed example in section 2.9.1, where we performed a NATD on $S^{3} \cong S U(2)$. That the metric on the $\mathrm{S}^{3} \cong S U(2)$ given in terms of the Maurer-Cartan forms $\sigma_{i}$ is now multiplied by a warp factor $\cos ^{2} \alpha$. These warp factors will now have to be included in the matrix $M_{i j}^{-1}$ in eq.(2.136) when we perform the NATD.

\footnotetext{
${ }^{1}$ Throughout this paper we will be using $2 \kappa_{10}^{2} T_{D_{p}}=(2 \pi)^{7-p} \alpha^{\prime(7-p) / 2}$ and $g_{s}=1$.
} 
The resulting type IIA solution then reads ${ }^{2}$

$$
\begin{aligned}
d s^{2} & =L^{2} d s_{A d S_{5}}^{2}+L^{2}\left(d \alpha^{2}+\sin ^{2} \alpha d \beta^{2}\right)+\frac{4 \alpha^{\prime 2}}{L^{2} \cos ^{2} \alpha} d \rho^{2}+\frac{4 L^{2} \alpha^{\prime 2} \rho^{2} \cos ^{2} \alpha}{\Delta} d \Omega_{2}^{2}(\chi, \xi) \\
B_{2} & =-\frac{16 \alpha^{\prime 3} \rho^{3}}{\Delta} d \Omega_{2}(\chi, \xi), \quad e^{-2 \phi}=\frac{L^{2} \cos ^{2} \alpha}{64 \alpha^{\prime 3}} \Delta, \\
F_{2} & =\frac{L^{4}}{2 \alpha^{\prime 3 / 2}} \cos ^{3} \alpha \sin \alpha d \alpha \wedge d \beta, \quad F_{4}=B_{2} \wedge F_{2},
\end{aligned}
$$

where

$$
\Delta=16 \alpha^{2} \rho^{2}+L^{4} \cos ^{4} \alpha .
$$

Note that the $A d S_{5}$ space remains unchanged, as do the $\alpha$ and $\beta$ directions. The NATD of the three-sphere is analogous to the one we obtained in our example in section 2.9.1, where the only change now comes from the warp factor of $\frac{1}{4} \cos ^{2} \alpha$ that was in front of the $S U(2) \subset$ $S O(6) \subset S U(2,2 \mid 4)$ of the original space. The $B_{2}$-field, dilaton, and RR-fluxes all change as explained in sections 2.8.1-2.9.

This solution is also known as the Sfetsos-Thompson solution, as it was first obtained in [190]. The solution is singular at $\alpha=\pi / 2$ due to the presence of NS5-branes. and fits inside the Gaiotto-Maldacena class of half-supersymmetric $\mathrm{AdS}_{5}$ geometries [190, 197] that we introduced in section 3.6.2. We commented in section 3.7 that the Sfetsos-Thompson background is a solution of this Gaiotto-Maldacena class with

$$
V_{N A T D}(z, \sigma)=z\left(\log \sigma-\frac{\sigma^{2}}{2}\right)+\frac{z^{3}}{3}, \quad \lambda(z)=z,
$$

and is dual to a $4 \mathrm{~d} \mathcal{N}=2 \mathrm{SCFT}$ with an infinitely long linear quiver.

Since the NATD preserves integrability by construction the superstring will be integrable on the resulting background, see also [140].

\subsubsection{NATD inside $A d S_{5}$}

When we apply a NATD on the non-compact subspace of the $A d S_{5} \times S^{5}$ solution (7.1) along the $S U(2)$ parametrised by the $\omega_{i}$ 's in eq. (7.4) we find a solution of type IIA given by [37]

$$
\begin{aligned}
d s^{2} & =L^{2}\left(-\cosh ^{2} r d t^{2}+d r^{2}\right)+\frac{4 \alpha^{\prime 2}}{L^{2} \sinh ^{2} r} d \rho_{1}^{2}+\frac{4 L^{2} \alpha^{\prime 2} \rho_{1}^{2} \sinh ^{2} r}{\tilde{\Delta}} d \Omega_{2}^{2}\left(\chi_{1}, \xi_{1}\right)+L^{2} d \Omega_{5}^{2}, \\
B_{2} & =-\frac{16 \alpha^{\prime 3} \rho_{1}^{3}}{\tilde{\Delta}} d \Omega_{2}\left(\chi_{1}, \xi_{1}\right), \quad e^{-2 \phi}=\frac{L^{2} \sinh ^{2} r}{64 \alpha^{\prime 3}} \tilde{\Delta} \\
F_{2} & =\frac{L^{4}}{2 \alpha^{\prime 3 / 2}} \cosh r \sinh ^{3} r d r \wedge d t, \quad F_{4}=B_{2} \wedge F_{2},
\end{aligned}
$$

\footnotetext{
${ }^{2}$ Notice that we are using a different normalisation to the one originally presented in [190].
} 


\section{T-DUALITY AS A SOLUTION GENERATING TECHNIQUE}

where

$$
\tilde{\Delta}=16 \alpha^{\prime 2} \rho_{1}^{2}+L^{4} \sinh ^{4} r .
$$

The singularity of this solution at $r=0$ is, as in the case of the previous Sfetsos-Thompson solution (7.7), due to the presence of NS5-branes. This solution has $\mathbb{R}_{t} \times S O(3) \times S O(6)$ isometries and fits into the Lin-Maldacena classification of half-BPS solutions of type IIA supergravity [40].

If we note in eq.(7.2) that the metric on the sphere maps to the metric on AdS when we send $\sin \alpha \rightarrow \cosh r, \cos \alpha \rightarrow \sinh r$ and $\beta \rightarrow i t$, we see that this resulting background is in the same way related to the Sfetsos-Thompson background on the previous section. However, it is worth noticing that this relation should be taken too literally since both solutions are rather different. The solution in eq.(7.7) can be associated with the near horizon setup of a D4-D5-NS5 system and is dual to a 4D SCFT with linear quiver structure [197]. In the solution of eq.(7.10) we break the conformal symmetries of the $\mathrm{AdS}_{5}$ subspace, and this solution will therefore not be dual to an SCFT. It is instead dual to a particular vacuum of the BMN matrix model [37].

\subsection{The Double NATD of $\mathrm{AdS}_{5} \times \mathrm{S}^{5}$}

Given the description of the above geometries that are obtained after applying one NATD in terms of half-BPS geometries that fit into different classifications, it is interesting to consider the application of two NATD's in both $S U(2)$ isometries of the $\mathrm{AdS}_{5}$ and the $\mathrm{S}^{5}$ subspaces.

We can either start with the solution in eq.(7.10) and perform a second NATD along the $S^{3} \cong S U(2)$ inside the remaining $S^{5}$, parameterised by the Maurer-Cartan forms $\sigma_{i}$ defined in eq.(7.4). Alternatively we could start from the solution in eq.(7.7) and perform a second NATD on the $S^{3}$ inside the remaining $\mathrm{AdS}_{5}$ space. In both cases we obtain the same solution, as the application of multiple NATD's on a space is 'commutative'. See also figure 7.1.

The resulting background is a solution of type IIB supergravity and has an NS-sector given by

$$
\begin{aligned}
d s^{2} & =L^{2}\left(-\cosh ^{2} r d t^{2}+d r^{2}+\frac{4 \alpha^{\prime 2}}{L^{4} \sinh ^{2} r} d \rho_{1}^{2}+\frac{4 \alpha^{\prime 2} \rho_{1}^{2} \sinh ^{2} r}{\tilde{\Delta}} d \Omega_{2}^{2}\left(\chi_{1}, \xi_{1}\right)\right. \\
& \left.+d \alpha^{2}+\sin ^{2} \alpha d \beta^{2}+\frac{4 \alpha^{\prime 2}}{L^{4} \cos ^{2} \alpha} d \rho^{2}+\frac{4 \alpha^{\prime 2} \rho^{2} \cos ^{2} \alpha}{\Delta} d \Omega_{2}^{2}(\chi, \xi)\right), \\
B_{2} & =-\frac{16 \alpha^{\prime 3} \rho^{3}}{\Delta} d \Omega_{2}(\chi, \xi)-\frac{16 \alpha^{\prime 3} \rho_{1}^{3}}{\tilde{\Delta}} d \Omega_{2}\left(\chi_{1}, \xi_{1}\right), \quad e^{-2 \phi}=\frac{L^{4} \cos ^{2} \alpha \sinh ^{2} r}{\left(64 \alpha^{\prime 3}\right)^{2}} \Delta \tilde{\Delta},
\end{aligned}
$$

where the functions $\Delta$ and $\tilde{\Delta}$ are the same as those defined in eqs. (7.8) and (7.12) respectively. This solution has two singular points at $r=0$ and $\alpha=\frac{\pi}{2}$ that correspond to two sets of NS 
fivebranes (we will indicate them by NS and NS') equally separated along the $\rho$ and $\rho_{1}$ axes, respectively. This fact will be verified later by studying the quantised charges of the solution.

Note the NS sector in eq.(7.13) is a linear combination of the NS-sectors in eqs.(7.7) and (7.10). This is because $\operatorname{AdS}_{5} \times S^{5}$ is a direct product of $\mathrm{AdS}_{5}$ and $\mathrm{S}_{5}$, and the NS-sectors in each of these subspaces is unaffected when we perform a NATD in the other subspace.

The RR sector of the resulting background in eq.(7.13) however mixes the RR sectors of eqs.(7.7) and (7.10) in a non-trivial way, and is of the form

$$
\begin{aligned}
F_{3}= & -\frac{L^{4}}{2 \alpha^{\prime}}\left(\rho \cosh r \sinh ^{3} r d r \wedge d t \wedge d \rho+\rho_{1} \sin \alpha \cos ^{3} \alpha d \alpha \wedge d \rho_{1} \wedge d \beta\right), \\
F_{5}= & \frac{L^{8} \rho^{2} \cos ^{4} \alpha \sinh ^{3} r \cosh r}{2 \Delta} d t \wedge d r \wedge d \rho \wedge d \Omega_{2}(\chi, \xi)+ \\
& \frac{8 \alpha^{\prime 2} L^{4} \rho \rho_{1}^{3} \sinh ^{3} r \cosh r}{\tilde{\Delta}} d r \wedge d t \wedge d \rho \wedge d \Omega_{2}\left(\chi_{1}, \xi_{1}\right)+ \\
& \frac{8 \alpha^{\prime 2} L^{4} \rho^{3} \rho_{1} \sin \alpha \cos ^{3} \alpha}{\Delta} d \alpha \wedge d \rho_{1} \wedge d \beta \wedge d \Omega_{2}(\chi, \xi)- \\
& \frac{L^{8} \rho_{1}^{2} \sin \alpha \cos ^{3} \alpha \sinh ^{4} r}{2 \tilde{\Delta}} d \alpha \wedge d \rho_{1} \wedge d \beta \wedge d \Omega_{2}\left(\chi_{1}, \xi_{1}\right), \\
F_{7}= & -\frac{8 L^{8} \alpha^{\prime 3} \rho^{2} \rho_{1}^{3} \cos ^{4} \alpha \cosh r \sinh ^{3} r}{\Delta \tilde{\Delta}} d t \wedge d r \wedge d \rho \wedge d \Omega_{2}(\chi, \xi) \wedge d \Omega_{2}\left(\chi_{1}, \xi_{1}\right)+ \\
& \frac{8 L^{8} \alpha^{\prime 3} \rho^{3} \rho_{1}^{2} \cos ^{3} \alpha \sin \alpha \sinh ^{4} r}{\Delta \tilde{\Delta}} d \beta \wedge d \alpha \wedge d \rho_{1} \wedge d \Omega_{2}(\chi, \xi) \wedge d \Omega_{2}\left(\chi_{1}, \xi_{1}\right) .
\end{aligned}
$$

For future reference we write the RR potentials associated with this solution satisfying $F_{p+1}=$ $d C_{p}-H_{3} \wedge C_{p-2}$. They are

$$
\begin{aligned}
C_{2}= & \frac{L^{4}}{8 \alpha^{\prime}}\left(-\rho \sinh ^{4} r d t \wedge d \rho+\rho_{1} \cos ^{4} \alpha d \rho_{1} \wedge d \beta\right) \\
C_{4}= & \frac{L^{4}}{8} \alpha^{\prime 2} \rho \rho_{1}\left(\frac{\rho_{1}^{2}}{\tilde{\Delta}} \sinh ^{4} r d t \wedge d \rho \wedge d \Omega_{2}\left(\chi_{1}, \xi_{1}\right)+\frac{\rho^{2}}{\Delta} \cos ^{4} \alpha d \beta \wedge d \rho_{1} \wedge d \Omega_{2}(\chi, \xi)\right) \\
- & \frac{L^{8}}{8} \cos ^{4} \alpha \sinh ^{4} r\left(\frac{\rho^{2}}{\Delta} d t \wedge d \rho \wedge d \Omega_{2}(\chi, \xi)+\frac{\rho_{1}^{2}}{\tilde{\Delta}} d \beta \wedge d \rho_{1} \wedge d \Omega_{2}\left(\chi_{1}, \xi_{1}\right)\right), \\
C_{6}= & 2 L^{6} \alpha^{\prime 2} \rho \rho_{1}\left(\frac{\rho_{1}^{2}}{\tilde{\Delta}} \sinh ^{3} r \cosh r \cot \alpha d t \wedge d r \wedge d \rho \wedge d \alpha \wedge d \Omega_{2}\left(\chi_{1}, \xi_{1}\right)\right. \\
& \left.+\frac{\rho^{2}}{\Delta} \cos ^{3} \alpha \sin \alpha \tanh r d \beta \wedge d \alpha \wedge d \rho_{1} \wedge d r \wedge d \Omega_{2}(\chi, \xi)\right) \\
& +\frac{2 L^{8} \alpha^{\prime 3}}{\Delta \tilde{\Delta}} \rho^{2} \rho_{1}^{2} \cos ^{4} \alpha \sinh ^{4} r\left(\rho d \beta \wedge d \rho_{1} \wedge d \Omega_{2}(\chi, \xi) \wedge d \Omega_{2}\left(\chi_{1}, \xi_{1}\right)\right. \\
& \left.+\rho_{1} d t \wedge d \rho \wedge d \Omega_{2}(\chi, \xi) \wedge d \Omega_{2}\left(\chi_{1}, \xi_{1}\right)\right) .
\end{aligned}
$$




\subsubsection{Supersymmetry of the solution}

Unlike the Abelian T-duality, the action of the NATD is non-reversable, by which we mean it breaks the isometry group on which we act with it. ${ }^{3}$ As an example we will consider how the NATD acts on $\mathrm{AdS}_{5} \times \mathrm{S}^{5}$, which can be constructed a the coset superspace

$$
\mathrm{AdS}_{5} \times S^{5} \cong \frac{S U(2,2 \mid 4)}{S O(1,4) \times S O(5)}
$$

If we act on either an $S U(2)$ isometry that is a subgroup of the $S O(2,4)$ group of Lorentz symmetries (as we did in section 7.0.3), or on one that is a subgroup of the $S O(6)$ internal symmetry group (as we did in section 7.0.2), part of this isometry group will be broken to either $S U(2 \mid 4)$ or $S U(2,2 \mid 2)$.

$$
\begin{gathered}
S U(2,2 \mid 4)=\left(\begin{array}{c|c}
S U(2,2) & F \\
\hline F & S U(4)
\end{array}\right) \\
S U(2 \mid 4)=\left(\begin{array}{cc|c}
0 & 0 & 0 \\
0 & S U(2) & f \\
\hline 0 & f & S U(4)
\end{array}\right),
\end{gathered}
$$

Notice that in both cases, the NATD breaks half of the fermionic elements of the isometry group. This means the number of Killing spinors of the resulting backgrounds are reduced by half, from 32 to 16.

If we were to perform the NATD on both the internal and Lorentz isometries the number of Killing spinors will be reduced by half, and we see from the structure of the supergroup there will only be and $S U(2 \mid 2)$ subgroup remaining

$$
S U(2 \mid 2)=\left(\begin{array}{cc|cc}
0 & 0 & 0 & 0 \\
0 & S U(2) & f & 0 \\
\hline 0 & f & S U(2) & 0 \\
0 & 0 & 0 & 0
\end{array}\right)
$$

\section{Explicit Calculation}

The above argument can be shown more explicitly by demonstrating that the $A d S_{5} \times S^{5}$ Killing spinors breaks supersymmetry when they are constrained to be independent of the $S U(2)$ angles inside the $A d S_{5}$ and $S^{5}$ subspaces. A procedure along these lines was already performed in [137] for the case where the spinor is independent of the $S U(2)$ angles inside $S^{5}$. Here we

\footnotetext{
${ }^{3}$ For an Abelian T-duality this is not the case, and we can recover the original space by T-dualising the dual geometry.
} 
will generalise that argument by imposing also the independence on the $S U(2)$ angles inside $A d S_{5}$. Following the results of [42], the number of supersymmetries preserved by this spinor will correspond to the ones after applying two NATD along the $S U(2)$ isometries of the $A d S_{5} \times S^{5}$ solution.

We first introduce a convenient set of frame fields for the solution in eq. (7.1) which explicitly exhibit the $\mathrm{SU}(2)$ isometries of the background. They are

$$
\begin{aligned}
e^{t} & =L \cosh r d t, \quad e^{r}=L d r, \quad e^{a}=\frac{1}{2} L \sinh r \omega_{a}, & a=3,4,5, \\
e^{\alpha} & =L d \alpha, \quad e^{\beta}=L \sin \alpha d \beta, \quad e^{i}=\frac{1}{2} L \cos \alpha \sigma_{i}, & i=6,7,8 .
\end{aligned}
$$

where $\omega_{i}$ and $\sigma_{i}$ were defined in eq.(7.4). In this basis the RR five form in eq.(7.5) reads

$$
F_{5}=\frac{4}{L}\left(e^{t} \wedge e^{r} \wedge e^{\theta_{1}} \wedge e^{\phi_{1}} \wedge e^{\psi_{1}}-e^{\theta} \wedge e^{\phi} \wedge e^{\psi} \wedge e^{\alpha} \wedge e^{\beta}\right) .
$$

Supersymmetric solutions impose the conditions ${ }^{4}$

$$
\begin{array}{ll}
\left(\nabla_{\mu}+\frac{i}{2 L} \Gamma^{\operatorname{tr} \theta_{1} \phi_{1} \psi_{1}} \Gamma_{\mu}\right) \epsilon=0, & \mu=t, r, \theta_{1}, \phi_{1}, \psi_{1}, \\
\left(\nabla_{\nu}-\frac{i}{2 L} \Gamma^{\theta \phi \psi \alpha \beta} \Gamma_{\nu}\right) \epsilon=0, & \nu=\theta, \phi, \psi, \alpha, \beta,
\end{array}
$$

where the Majorana-Weyl spinor $\epsilon$ satisfies

$$
\Gamma^{\operatorname{tr} \theta_{1} \phi_{1} \psi_{1} \theta \phi \psi \alpha \beta} \epsilon=-\epsilon .
$$

We are interested in solutions to eqs. (7.22) and (7.23) which are consistent with the independence of the spinor on the $S U(2)$ angles in both $A d S_{5}$ and $S^{5}$. By imposing these conditions we find the equations

$$
\begin{array}{ll}
\left(\Gamma^{\phi_{1} \psi_{1}}-\cosh r \Gamma^{r, \theta_{1}}-i \sinh r \Gamma^{t r \phi_{1} \psi_{1}}\right) \epsilon=0, & \left(\Gamma^{\phi \psi}-\sin \alpha \Gamma^{\theta, \psi}-i \cos \alpha \Gamma^{\phi \psi \alpha \beta}\right) \epsilon=0, \\
\left(\Gamma^{\theta_{1} \psi_{1}}+\cosh r \Gamma^{r, \phi_{1}}-i \sinh r \Gamma^{t r \theta_{1} \psi_{1}}\right) \epsilon=0, & \left(\Gamma^{\theta \psi}+\sin \alpha \Gamma^{\phi, \alpha}-i \cos \alpha \Gamma^{\theta \psi \alpha \beta}\right) \epsilon=0, \\
\left(\Gamma^{\theta_{1} \phi_{1}}-\cosh r \Gamma^{r, \psi_{1}}-i \sinh r \Gamma^{t r \theta_{1} \phi_{1}}\right) \epsilon=0, & \left(\Gamma^{\theta \phi}-\sin \alpha \Gamma^{\psi, \alpha}-i \cos \alpha \Gamma^{\theta \phi \alpha \beta}\right) \epsilon=0, \\
\left(2 \partial_{t}+\sinh r \Gamma^{t r}-i \cosh r \Gamma^{r \theta_{1} \phi_{1} \psi_{1}}\right) \epsilon=0, & \left(2 \partial_{\beta}-\cos \alpha \Gamma^{\alpha \beta}-i \sin \alpha \Gamma^{\theta \phi \alpha \beta}\right) \epsilon=0, \\
\left(2 \partial_{r}+i \Gamma^{t \theta_{1} \phi_{1} \psi_{1}}\right) \epsilon=0, & \left(2 \partial_{\alpha}+i \Gamma^{t \theta \phi \psi \beta}\right) \epsilon=0,
\end{array}
$$

These equations can be massaged giving rise to the following set of equations

$$
\begin{array}{ll}
2 \partial_{\beta} \epsilon+i \epsilon=0, & 2 \partial_{t} \epsilon-i \epsilon=0, \\
2 \partial_{\alpha} \epsilon+i \Gamma^{\theta \phi \psi \beta} \epsilon=0, & 2 \partial_{r} \epsilon-i \Gamma^{t \theta_{1} \phi_{1} \psi_{1}} \epsilon=0,
\end{array}
$$

\footnotetext{
${ }^{4}$ We have written the spinor in complex notation as $\epsilon=\epsilon_{1}+i \epsilon_{2}$
} 
and the projector conditions

$$
\begin{aligned}
\Gamma^{\alpha \beta} \epsilon & =\left(-i \cos \alpha+\sin \alpha \Gamma^{\theta \phi \psi \beta}\right) \epsilon=i e^{i \alpha \Gamma^{\theta \phi \psi \beta}} \epsilon, \\
\Gamma^{r \theta_{1} \phi_{1} \psi_{1}} \epsilon & =\left(-\cosh r+i \sinh r \Gamma^{t \theta_{1} \phi_{1} \psi_{1}}\right) \epsilon=-e^{i t \Gamma^{\theta \phi \psi \beta}} \epsilon .
\end{aligned}
$$

The Killing spinor is easily found and reads

$$
\epsilon=e^{\frac{i}{2}(t-\beta)} e^{\frac{i}{2}\left(r \Gamma^{t \theta_{1} \phi_{1} \psi_{1}}-\alpha \Gamma^{\theta \phi \psi \beta}\right)} \epsilon_{0}
$$

where $\epsilon_{0}$ is a spinor satisfying the projections

$$
\Gamma^{\alpha \beta} \epsilon_{0}=-i \epsilon_{0}, \quad \Gamma^{r \theta_{1} \phi_{1} \psi_{1}} \epsilon_{0}=-\epsilon_{0} .
$$

Since these projectors commute with each other, we have a total of eight supercharges preserved by the solution. Had we started by imposing independence of the spinor on the Hopf-fibre angles of the $S^{3}$ inside $A d S_{5}$ and $S^{5}, \partial_{\psi_{1}} \epsilon=0, \partial_{\psi} \epsilon=0$, we would have obtained the same set of projections, ensuring that the solution after two Abelian T-dualities along the Hopf-fibre angles preserves also eight supercharges [43].

\subsubsection{Quantised Charges}

In this section we shall study the information obtained from the Page charges of the solution in eqs.(7.13) and (7.14).

We start by noticing that a common feature of many $S U(2)$ NATD solutions is the presence of singular points in the geometry. Such singularities correspond to the points where the $S^{3}$ along which we are dualising the background shrinks to zero size and are originates from the presence of NS5-branes. For the solution in eq.(7.13) we see that there are two such singular

points, at $r=0$ and at $\alpha=\frac{\pi}{2}$. The leading order behaviour of the metric and dilaton close to these points are

$$
\begin{gathered}
d s^{2}=L^{2}\left(-d t^{2}+d \beta^{2}+\frac{1}{4 \nu}\left(d{\tilde{\rho_{1}}}^{2}+d \nu^{2}+\nu^{2} d \Omega_{2}^{2}\left(\chi_{1}, \xi_{1}\right)\right)+\frac{1}{4 \mu}\left(d \tilde{\rho}^{2}+d \mu^{2}+\mu^{2} d \Omega_{2}^{2}(\chi, \xi)\right)\right) \\
e^{\Phi} \sim \frac{4 \alpha^{\prime}}{L^{2} \sqrt{\mu \nu}} \frac{1}{\rho \rho_{1}}
\end{gathered}
$$

where $\nu=r^{2}, \mu=\left(\alpha-\frac{\pi}{2}\right)^{2}, \tilde{\rho}=16 \alpha^{\prime 2} / L^{4} \rho$ and the same for $\tilde{\rho}_{1}$. The metric in eq. (7.32) can be thought of as a continuous distribution of NS and NS' fivebranes along the $\tilde{\rho}_{1}$ and $\tilde{\rho}$ directions. This can also be verified by measuring the units of $H_{3}$ flux through the relevant cycles. Close to $r=0, \alpha=\pi / 2$ the leading order behaviour of the $B_{2}$ field is $B_{2} \sim \alpha^{\prime} \rho_{1} d \Omega_{2}\left(\chi_{1}, \xi_{1}\right)+$ $\alpha^{\prime} \rho d \Omega_{2}(\chi, \xi)$. In $[45,44]$ an argument was proposed to bound the non-compact coordinate 
appearing in the NATD solutions, in the case at hand $\rho_{1}$ and $\rho$, such that the number of fivebranes is finite. The argument relies on the boundedness of the quantity

$$
b_{0}=\frac{1}{4 \pi^{2} \alpha^{\prime}} \int_{\Sigma} B_{2} \quad \in[0,1] .
$$

In the present case we have two non-trivial two-cycles given by $S_{\left(\chi_{1}, \xi_{1}\right)}^{2}$ and $S_{(\chi, \xi)}^{2}$ close to $r=0$ and $\alpha=\frac{\pi}{2}$ respectively. Hence, according to [45, 44], in order to achieve $b_{0} \in[0,1]$ over the above two cycles we have to impose simultaneously that $\rho_{1} \in[0, \pi]$ and $\rho \in[0, \pi]$, which makes the $\left(\rho_{1}, \rho\right)$-plane a grid of size $\pi$, the boundary of which is delimited by NS5-branes, denoted by NS5 and NS5'. We then see that in order to fully cover the non-compact range of $\rho, \rho_{1} \in \mathbb{R}^{+}$ and keep $b_{0} \in[0,1]$, a large gauge transformation of the form

$$
B_{2} \rightarrow \hat{B}_{2}=B_{2}-n_{1} \pi d \Omega_{2}\left(\chi_{1}, \xi_{1}\right)-n \pi d \Omega_{2}(\chi, \xi),
$$

is required whenever we pass through the intervals $\left[n_{1} \pi,\left(n_{1}+1\right) \pi\right]$ and $[n \pi,(n+1) \pi]$. Therefore, the $H_{3}$ flux through the cycles $\left.\left(\rho_{1}, S_{\left(\chi_{1}, \xi_{1}\right)}^{2}\right)\right|_{r=0}$ and $\left.\left(\rho, S_{(\chi, \xi)}^{2}\right)\right|_{\alpha=\frac{\pi}{2}}$ is, respectively,

$$
N_{N S 5}=\frac{1}{4 \pi^{2} \alpha^{\prime}} \int_{S_{\left(\chi_{1}, \xi_{1}\right)}^{2}} \int_{0}^{n_{1} \pi} H_{3}=n_{1}, \quad N_{N S 5^{\prime}}=\frac{1}{4 \pi^{2} \alpha^{\prime}} \int_{S_{(\chi, \xi)}^{2}} \int_{0}^{n \pi} H_{3}=n .
$$

We then have NS5-branes located at positions $\rho_{1}=\pi, 2 \pi \ldots n_{1} \pi$ with their worldvolume along $(t, \alpha, \beta, \rho, \chi, \xi)$ and NS5' branes at $\rho=\pi, 2 \pi \ldots n \pi$ extended along $\left(t, \beta, r, \rho_{1}, \chi_{1}, \xi_{1}\right)$. These two sets of NS5-branes divide the entire $\left(\rho_{1}, \rho\right)$-plane into a grid. Notice that, the above discussed configuration of NS5-branes in $\left(\rho_{1}, \rho\right)$ is a consequence that the $B_{2}$ field in (7.13) is a superposition of the $B_{2}$ fields in (7.7) and (7.10).

In addition we have non-zero $F_{3}, F_{5}$ and $F_{7} \mathrm{RR}$ field strengths, so that we can measure the flux of these fields through non-trivial cycles present in the geometry. We have four piecewise compact cycles defined by

$$
\begin{array}{ll}
\Sigma_{3}=\left(\rho_{1}, S_{(\alpha, \beta)}^{2}\right), & \Sigma_{5}^{\prime}=\Sigma_{3} \otimes S_{\left(\xi_{1}, \chi_{1}\right)}^{2}, \\
\Sigma_{5}=\Sigma_{3} \otimes S_{(\xi, \chi)}^{2}, & \Sigma_{7}=\Sigma_{3} \otimes S_{\left(\xi_{1}, \chi_{1}\right)}^{2} \otimes S_{(\xi, \chi)}^{2} .
\end{array}
$$

The D5 Page charge computed in the interval $\rho_{1} \in\left[n_{1} \pi,\left(n_{1}+1\right) \pi\right]$ is

$$
N_{D 5}=\frac{1}{4 \pi^{2} \alpha^{\prime}} \int_{\Sigma_{3}} F_{3}=\frac{L^{4} \pi}{32 \alpha^{\prime 2}}\left(1+2 n_{1}\right)
$$

where the D5-branes are extended along $\left(t, \rho, \chi_{1}, \xi_{1}, \chi, \xi\right)$. Moreover, Page charge for D1 and D3-branes is induced due to large gauge transformations on the $B_{2}$-field. In the interval $\rho_{1} \in$ 
$\left[n_{1} \pi,\left(n_{1}+1\right) \pi\right]$ and $\rho \in[n \pi,(n+1) \pi]$, these Page charges are

$$
\begin{aligned}
N_{D 3^{\prime}} & =\frac{1}{(2 \pi)^{4} \alpha^{2}} \int_{\Sigma_{5}^{\prime}} F_{5}-F_{3} \wedge \hat{B}_{2}=\frac{L^{4} \pi}{48 \alpha^{\prime 2}}\left(3 n_{1}^{2}+3 n_{1}+1\right)+n_{1} N_{D 5} \\
N_{D 3} & =\frac{1}{(2 \pi)^{4} \alpha^{2}} \int_{\Sigma_{5}} F_{5}-F_{3} \wedge \hat{B}_{2}=n \frac{L^{4} \pi}{32 \alpha^{\prime 2}}\left(1+2 n_{1}\right)=n N_{D 5} \\
N_{D 1} & =\frac{1}{(2 \pi)^{6} \alpha^{\prime 3}} \int_{\Sigma_{7}} F_{7}-F_{5} \wedge \hat{B}_{2}+\frac{1}{2} F_{3} \wedge \hat{B}_{2} \wedge \hat{B}_{2}=n_{1} N_{D_{3}}+n \frac{L^{4} \pi}{48 \alpha^{\prime 2}}\left(1-3 n_{1}^{2}\right) .
\end{aligned}
$$

We find there are two sets of D3-branes, one with worldvolume coordinates $(t, \rho, \chi, \xi)$ (denoted by D3'), and one along $\left(t, \rho, \chi_{1}, \xi_{1}\right)$ (denoted by D3). From eqs.(7.39) and (7.40) we see there is a contribution of the D1-branes to the D3 charge coming from the term,

$$
N_{D 1}^{(d)}=\frac{n n_{1}}{2^{6} \pi^{4} \alpha^{\prime 3}} \int_{\Sigma_{5}} F_{3\left(\rho_{1}, \alpha, \beta\right)} d \Omega_{2}(\chi, \xi) \int_{S_{\left(\xi_{1}, \chi_{1}\right)}^{2}} d \Omega_{2}\left(\chi_{1}, \xi_{1}\right)=n_{1} N_{D 3} .
$$

Therefore we see that this fraction of the D1 charge is dissolved into the D3-branes. This suggest that we have D1-branes expanded onto spherical D3-branes due to the presence of the $B_{2\left(\chi_{1}, \xi_{1}\right)}$ field, as a consequence of the Myers dielectric effect [46]. The rest of the charge is usual D1-brane charge. Notice that, from eq. (7.39), one might think that the D3-branes will, in addition, blow up into D5-branes. This, however, is not possible as the D3 would then blow up onto an $\Omega_{2}(\chi, \xi) 2$-sphere of radius $\rho$, which is not possible, as the $\rho$-coordinate is part of the worldvolume of the D3-branes. This effect is similar to the one found in [37] in which D0-branes expanded onto fuzzy two-spheres to form spherical D2-branes. In analogy to this case, we will identify below $\rho_{1}$ as the radius of the expanded D1-branes into $S_{\left(\chi_{1}, \xi_{1}\right)}^{2}$.

Moreover, the number $N_{D 3^{\prime}}$ of D3' branes increases every time we go through the interval $\rho_{1} \in\left[n_{1} \pi,\left(n_{1}+1\right) \pi\right]$. From eq. (7.38) we see that a fraction,

$$
N_{D 3^{\prime}}^{(d)}=\frac{1}{(2 \pi)^{4} \alpha^{\prime 2}} \int_{\Sigma_{5}^{\prime}} F_{3\left(\rho_{1}, \alpha, \beta\right)} \int_{S_{\left(\xi_{1}, \chi_{1}\right)}^{2}} d \Omega_{2}\left(\chi_{1}, \xi_{1}\right)=n_{1} N_{D 5},
$$

of this D3' charge is dissolved in the D5-branes. Again, the remaining charge is usual D3' charge. From eqs. (7.41) and (7.42), the total, dissolved, charge carried by $k$ concentric D3 and D5-branes is

$$
N_{D 1}^{(d)}=\sum_{n_{1}=1}^{k} n_{1} N_{D_{3}}, \quad N_{D 3^{\prime}}^{(d)}=\sum_{n_{1}=1}^{k} n_{1} N_{D_{5}} .
$$

The above relations indicate that the vacua of the theory correspond to reducible $S U(2)$ representations where $n_{1}$, associated to the radius of the spherical branes, corresponds to the dimension of the representation whilst $N_{D_{3}}$ and $N_{D_{5}}$ are their multiplicities. This is similar to the vacua of the $\mathcal{N}=1^{*}$ theory studied by Polchinsky and Strassler [47]. 


\begin{tabular}{c||cc|ccc|cc|ccc} 
& $t$ & $r$ & $\rho_{1}$ & $\chi_{1}$ & $\xi_{1}$ & $\alpha$ & $\beta$ & $\rho$ & $\chi$ & $\xi$ \\
\hline \hline NS5 & $\bullet$ & $\bullet$ & $\bullet$ & $\bullet$ & $\bullet$ & $\cdot$ & $\bullet$ & $\cdot$ & $\cdot$ & $\cdot$ \\
\hline NS5 & $\bullet$ & $\cdot$ & $\cdot$ & $\cdot$ & $\cdot$ & $\bullet$ & $\bullet$ & $\bullet$ & $\bullet$ & $\bullet$ \\
\hline \hline D5 & $\bullet$ & $\cdot$ & $\cdot$ & $\bullet$ & $\bullet$ & $\cdot$ & $\cdot$ & $\bullet$ & $\bullet$ & $\bullet$ \\
\hline D3' & $\bullet$ & $\cdot$ & $\cdot$ & $\cdot$ & $\cdot$ & $\cdot$ & $\cdot$ & $\bullet$ & $\bullet$ & $\bullet$ \\
\hline \hline D3 & $\bullet$ & $\cdot$ & $\cdot$ & $\bullet$ & $\bullet$ & $\cdot$ & $\cdot$ & $\bullet$ & $\cdot$ & $\cdot$ \\
\hline D1 & $\bullet$ & $\cdot$ & $\cdot$ & $\cdot$ & $\cdot$ & $\cdot$ & $\cdot$ & $\bullet$ & $\cdot$ & $\cdot$
\end{tabular}

Table 7.1: Overview of the brane content of the Type IIB supergravity solution in eqs. (7.13)(7.14), with the directions along which the branes are suspended.

We summarise the brane content of our solution in Table 7.1. From this table we see that all D-branes in our solution intersect each other only along the $t$ and $\rho$-directions. We therefore expect that the resulting worldvolume theory of the intersecting branes is a $(1+1)$-dimensional quantum field theory. However, since the D-branes have finite length along the $\rho$-direction, at low enough energies this theory is described by a $(0+1)$-dimensional theory, a matrix model.

In conclusion, we have two arrays of NS5-branes forming a grid in the $\left(\rho, \rho_{1}\right)$-plane. The first set, denoted by NS5, is located at $\rho_{1}=\pi, 2 \pi, \ldots, n_{1} \pi$ and is extended along $(t, \alpha, \beta, \rho, \chi, \xi)$ whilst the second set, denoted by NS5', located at $\rho=\pi, 2 \pi, \ldots, n \pi$ is extended along $\left(t, r, \beta, \rho_{1}, \chi_{1}, \xi_{1}\right)$. In addition, as a consequence of large gauge transformations, the D1 and D3'-branes expand onto fuzzy two-spheres with equilibrium radius of $\rho_{1}=n_{1} \pi$ giving rise to D3 and D5-branes. Moreover at any value of $\rho_{1}=n_{1} \pi$ we have usual D1 and D3' charge. We will see in the next section that these branes are indeed BPS.

To close this section, some comments about IIB matrix models are now in order. Matrix models constructed upon the plane wave background in IIB supergravity have been studied in the literature following different approaches $[48,49,50,54,55]$. For instance, the author in [49] considered the quantisation of the 3-brane action whereas the starting point in [50] was to consider the action for coincident gravitons [56] for the maximally supersymmetric IIB pp-wave background. In the former case, the model contains eight transverse non-Abelian scalars which explicitly realise the $S O(4) \times S O(4)$ symmetry of the pp-wave background whilst in the latter the fuzzy 3 -sphere vacua were constructed as $S^{1}$ fibrations over fuzzy 2 -spheres which break the isometries of the model down to $(S O(3) \times U(1))^{2}$. The main difference of these models with the matrix model studied here is that in the present case there is only one set of three non-Abelian scalars which correspond to the (fuzzy) $\Omega_{2}\left(\chi_{1}, \xi_{1}\right)$ where the D1 and D3 expand into D3' and D5-branes respectively. It would be interesting to understand if the matrix model studied here is embedded as a particular case of the above described matrix models. 


\subsubsection{Dielectric branes}

In this section we will study D3 and D5 probe branes in the background of the NATD solution of eqs.(7.13)-(7.14). We will show that such probe branes correspond to spherical branes carrying D1 and D3' charge respectively, and have radii $n_{1} \pi$, proportional to their charge. This supports the explanation of the brane set-up characterising the NATD solution studied in the preceding section.

\section{Dielectric D3 brane}

Consider a D3-brane with worldvolume along $\left(t, \rho, \chi_{1}, \xi_{1}\right)$. Its induced metric is of the form

$$
d s_{D 3}^{2}=L^{2}\left(-\cosh ^{2} r d t^{2}+\frac{4 \alpha^{2}}{L^{4} \cos ^{2} \alpha} d \rho^{2}+\frac{4 \alpha^{\prime 2} \rho_{1}^{2} \sinh ^{2} r}{\tilde{\Delta}} d \Omega_{2}^{2}\left(\chi_{1}, \xi_{1}\right)\right) .
$$

Large gauge transformations induce D1 charge proportional to $n_{1} \pi$ to this D3-brane, as can be seen from the WZ action for the D3-brane,

$$
\begin{aligned}
S_{D 3}^{W Z} & =T_{D_{3}} \int_{\left(t, \rho, S_{\left(\chi_{1}, \xi_{1}\right)}^{2}\right)} C_{4}-C_{2} \wedge B_{2} \\
& =T_{D_{3}} \alpha^{\prime} n_{1} \pi \int_{\left(t, \rho, S_{\left(\chi_{1}, \xi_{1}\right)}^{2}\right)} C_{2(t, \rho)} d t d \rho d \Omega_{2}\left(\chi_{1}, \xi_{1}\right), \\
& =T_{D_{3}} \frac{L^{4} n_{1} \pi^{2} \sinh ^{4} r}{2} \int_{\mathbb{R}_{t}} \int_{n \pi}^{(n+1) \pi} d \rho \rho,
\end{aligned}
$$

where $C_{2(t, \rho)}$ is the $(t, \rho)$ component of the $C_{2}$ potential in eq. (7.15). For the DBI action we find

$$
\begin{aligned}
S_{D 3}^{D B I}= & T_{D_{3}} \int_{t, \rho, S_{\left(\chi_{1}, \xi_{1}\right)}} d^{4} x e^{-\Phi} \sqrt{-\operatorname{det}(g-\mathcal{F})}, \\
= & T_{D_{3}} \frac{L^{2} \pi \sinh ^{3} r \cosh r}{8 \alpha^{\prime}} \int_{\mathbb{R}_{t}} \int_{n \pi}^{(n+1) \pi} d \rho \sqrt{16 \alpha^{\prime 2} \rho^{2}+L^{4} \cos ^{4} \alpha} \\
& \times \sqrt{16 \alpha^{\prime 2}\left(\rho_{1}-n_{1} \pi\right)^{2} \rho_{1}^{2}+\left(n_{1} \pi\right)^{2} L^{4} \sinh ^{4} r} .
\end{aligned}
$$

We easily see that the D3-brane finds its equilibrium position by sitting at the points $\alpha=\pi / 2$, $\rho_{1}=n_{1} \pi$ where the DBI action reduces to

$$
S_{D 3}^{D B I}=T_{D_{3}} \frac{L^{4} n_{1} \pi^{2} \sinh r \cosh r}{2} \int_{\mathbb{R}_{t}} \int_{n \pi}^{(n+1) \pi} d \rho \rho .
$$


We find that close to $r \sim \infty$ the D3-brane becomes BPS as the leading order terms in the DBI and WZ actions exactly cancel. In this limit the D3 extending on $S_{\left(\chi_{1}, \xi_{1}\right)}^{2}$ sits at the singularity at $\alpha=\pi / 2$ and has a radius

$$
R \sim \frac{2 n_{1} \pi}{L \sinh r}
$$

We can repeat the above computation for a probe D3'-brane along $(t, \rho, \chi, \xi)$. However, this does not correspond to a dielectric brane as the minimum of the potential felt by this D3'-brane sets its equilibrium radius, $\rho_{1}$, to zero. This is to be expected since the radius of the $S_{(\chi, \xi)}^{2}$ is $\rho$, which is part of the worldvolume coordinates of the D3'-brane. We will see in the next subsection however that D3'-branes can polarise into spherical D5-branes.

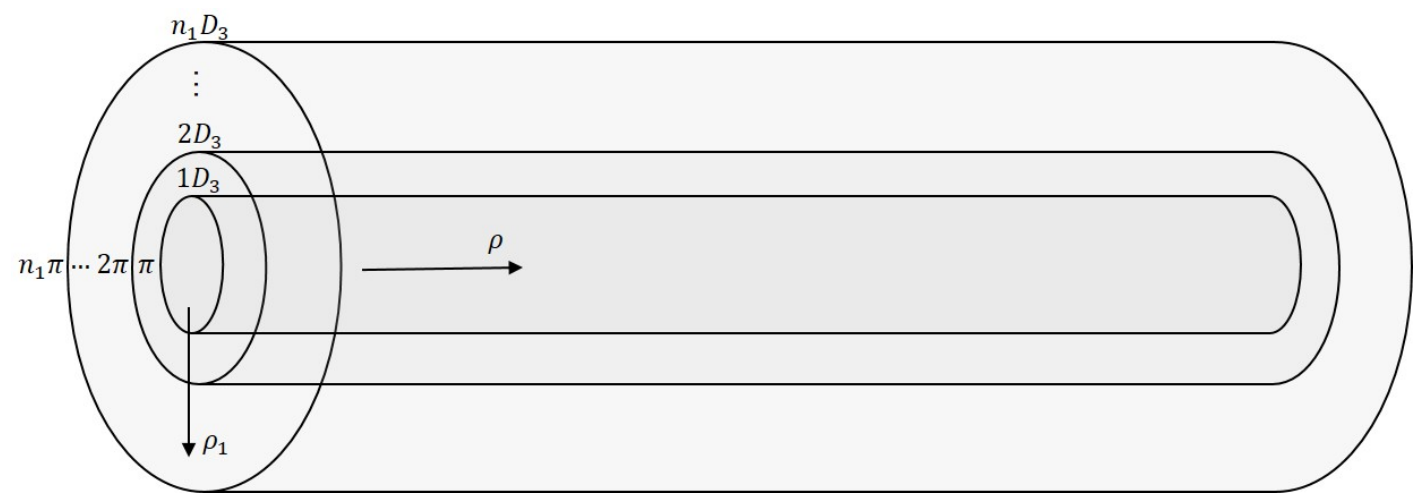

Figure 7.2: Expanded D3-branes on 2-spheres with radii $\rho_{1}=n_{1} \pi$.

\section{Dielectric D5 brane}

Next we consider a probe D5-brane with worldvolume coordinates along $\left(t, \rho, \chi, \xi, \chi_{1}, \xi_{1}\right)$, wrapping the $S^{2}$ along $\left(\chi_{1}, \xi_{1}\right)$. The induced metric on this D5-brane is

$d s_{D 5}^{2}=L^{2}\left(-\cosh ^{2} r d t^{2}+\frac{4 \alpha^{\prime 2}}{L^{4} \cos ^{2} \alpha} d \rho^{2}+\frac{4 \alpha^{\prime 2} \rho^{2} \cos ^{2} \alpha}{\Delta} d \Omega_{2}^{2}(\chi, \xi)+\frac{4 \alpha^{\prime 2} \rho_{1}^{2} \sinh ^{2} r}{\tilde{\Delta}} d \Omega_{2}^{2}\left(\chi_{1}, \xi_{1}\right)\right)$

The DBI and WZ actions for this probe brane, in the presence of large gauge transformations, are respectively

$$
\begin{aligned}
S_{D 5}^{D B I}= & -\frac{T_{D_{5}} L^{2} \pi^{2}}{2} \int_{\mathbb{R}_{t}} \int_{n \pi}^{(n+1) \pi} d t d \rho \cosh r \sinh r \sqrt{16 \alpha^{\prime 2}\left(\rho_{1}-n_{1} \pi\right)^{2} \rho_{1}^{2}+L^{4}\left(n_{1} \pi\right)^{2} \sinh ^{4} r} \\
& \times \sqrt{16 \alpha^{\prime 2}(\rho-n \pi)^{2} \rho^{2}+L^{4}(n \pi)^{2} \cos ^{4} \alpha} \\
S_{D 5}^{W Z} & =T_{D_{5}} \int_{\left(t, \rho, S_{(\chi, \xi)}^{2}, S_{\left(\chi_{1}, \xi_{1}\right)}^{2}\right)}\left(C_{4}-C_{2} \wedge B_{2}+\frac{1}{2} C_{2} \wedge \Delta B_{2}\right) \wedge \Delta B_{2}
\end{aligned}
$$


where $\Delta B_{2}=n_{1} \pi d \Omega_{2}\left(\chi_{1}, \xi_{1}\right)+n \pi d \Omega_{2}(\chi, \xi)$. From the form of the WZ action we see that the D5-brane carries both D1 and D3'-brane charges. Their separate contributions using the RR potentials (7.15)-(7.17) are

$$
\begin{aligned}
T_{D_{5}} \int\left(C_{4}-C_{2} \wedge B_{2}\right) \wedge \Delta B_{2} & =-2\left(n_{1} \pi\right) T_{D_{5}} L^{4} \pi^{2} \alpha^{\prime} \sinh ^{4} r \int_{\mathbb{R}_{t}} \int_{n \pi}^{(n+1) \pi} d \rho \rho^{2}, \\
T_{D_{5}} \int C_{2} \wedge \Delta B_{2} \wedge \Delta B_{2} & =2\left(n_{1} \pi\right) T_{D_{5}} L^{4} \pi^{2} \alpha^{\prime} \sinh ^{4} r \int_{\mathbb{R}_{t}} \int_{n \pi}^{(n+1) \pi} n \pi d \rho \rho .
\end{aligned}
$$

We see that the charge of both terms is proportional to $n_{1} \pi$. The D5-brane is in equilibrium at $\rho_{1}=n_{1} \pi, \alpha=\frac{\pi}{2}$ and $r \sim \infty$ when both the WZ and DBI actions are of the same form and cancel each other. However, notice that at $\alpha=\pi / 2$ the $\Omega_{2}(\chi, \xi)$ shrinks to zero size and the D5-brane becomes effectively the D3-brane studied in section 7.1.3. Moreover, if we instead take $n=0$ together with $\rho_{1}=n_{1} \pi$ and $r \sim \infty$ the D5-brane is indeed stable because of its D3' charge. The D5-brane therefore wraps an $S_{\left(\chi_{1}, \xi_{1}\right)}^{2}$ of radius (7.48) carrying D3' charge proportional to its radius. 
BIBLIOGRAPHY

\section{Bibliography}

[1] P. D. Lax, "Integrals of Nonlinear Equations of Evolution and Solitary Waves", Commun. Pure Appl. Math. 21, 467 (1968).

[2] A. Torrielli, "Lectures on Classical Integrability", J. Phys. A 49 (2016) no.32, 323001 [arXiv:1606.02946 [hep-th]].

[3] J. M. Maldacena, The Large $N$ limit of superconformal field theories and supergravity, Int. J. Theor. Phys. 38, 1113 (1999), [hep-th/9711200].

[4] G. 't Hooft, "A Planar Diagram Theory for Strong Interactions", Nucl. Phys. B 72, 461 (1974).

[5] J. A. Minahan, "Review of AdS/CFT Integrability, Chapter I.1: Spin Chains in N=4 Super Yang-Mills", Lett. Math. Phys. 99, 33 (2012) [arXiv:1012.3983 [hep-th]].

[6] I. Bena, J. Polchinski and R. Roiban, "Hidden symmetries of the AdS(5) $x S^{* * 5}$ superstring", Phys. Rev. D 69, 046002 (2004) [hep-th/0305116].

[7] N. Beisert and M. Staudacher, "The N=4 SYM integrable super spin chain", Nucl. Phys. B 670, 439 (2003) [hep-th/0307042].

[8] O. Aharony, O. Bergman, D. L. Jafferis and J. Maldacena, " $N=6$ superconformal ChernSimons-matter theories, M2-branes and their gravity duals", JHEP 0810, 091 (2008) [arXiv:0806.1218 [hep-th]].

[9] G. Arutyunov and S. Frolov, "Foundations of the $A d S_{5} \times S^{5}$ Superstring. Part I" J. Phys. A 42, 254003 (2009) [arXiv:0901.4937 [hep-th]].

[10] N. Beisert et al., "Review of AdS/CFT Integrability: An Overview", Lett. Math. Phys. 99, 3 (2012) [arXiv:1012.3982 [hep-th]].

[11] G. Arutyunov and S. Frolov, "Superstrings on $A d S_{4} \times C_{3}$ as a Coset Sigma-model", JHEP 0809, 129 (2008) [arXiv:0806.4940 [hep-th]]. 
[12] D. Bak and S. J. Rey, "Integrable Spin Chain in Superconformal Chern-Simons Theory", JHEP 0810, 053 (2008) [arXiv:0807.2063 [hep-th]].

[13] D. Gepner and E. Witten, "String Theory on Group Manifolds", Nucl. Phys. B 278, 493 (1986).

[14] E. Witten, "Nonabelian Bosonization in Two-Dimensions" Commun. Math. Phys. 92, 455 (1984).

[15] P. Ramond, "Dual Theory for Free Fermions", Phys. Rev. D 3, 2415 (1971).

[16] A. Neveu and J. H. Schwarz, "Quark Model of Dual Pions", Phys. Rev. D 4, 1109 (1971).

[17] F. Gliozzi, J. Scherk and D. I. Olive, "Supersymmetry, Supergravity Theories and the Dual Spinor Model", Nucl. Phys. B 122, 253 (1977).

[18] M. B. Green and J. H. Schwarz, "Covariant Description of Superstrings", Phys. Lett. 136B, 367 (1984).

[19] P. A. Grassi and M. Marescotti, "Flux vacua and supermanifolds", JHEP 0701, 068 (2007) [hep-th/0607243].

[20] J. de Boer, P. A. Grassi and P. van Nieuwenhuizen, "Noncommutative superspace from string theory", Phys. Lett. B 574, 98 (2003) [hep-th/0302078].

[21] M. T. Grisaru, H. Nishino and D. Zanon, "Beta Functions for the Green-schwarz Superstring", Nucl. Phys. B 314, 363 (1989).

[22] M. T. Grisaru, H. Nishino and D. Zanon, "Beta Function Approach to the Green-schwarz Superstring", Phys. Lett. B 206, 625 (1988).

[23] I. N. McArthur, "Kappa symmetry of Green-Schwarz actions in coset superspaces", Nucl. Phys. B 573, 811 (2000) [hep-th/9908045].

[24] M. Henneaux and L. Mezincescu, "A Sigma Model Interpretation of Green-Schwarz Covariant Superstring Action", Phys. Lett. 152B, 340 (1985).

[25] T. H. Buscher, "A Symmetry of the String Background Field Equations", Phys. Lett. B 194, 59 (1987).

[26] T. H. Buscher, "Path Integral Derivation of Quantum Duality in Nonlinear Sigma Models", Phys. Lett. B 201, 466 (1988).

[27] For a review see: L. Alvarez-Gaume and F. Zamora, Duality in quantum field theory and string theory, AIP Conf. Proc. 423, no. 1, 46 (1998), [hep-th/9709180]. 
[28] E. Alvarez, L. Alvarez-Gaume and Y. Lozano, "A Canonical approach to duality transformations", Phys. Lett. B 336, 183 (1994) [hep-th/9406206].

[29] J. Polchinski, Dualities of Fields and Strings, Stud. Hist. Philos. Mod. Phys. 59, 6 (2017), [arXiv:1412.5704 [hep-th]].

[30] N. A. Obers and B. Pioline, U duality and M theory, Phys. Rept. 318, 113 (1999), [hepth/9809039].

[31] G. 't Hooft, A Planar Diagram Theory for Strong Interactions, Nucl. Phys. B 72, 461 (1974).

[32] X. C. de la Ossa and F. Quevedo, Duality symmetries from nonAbelian isometries in string theory, Nucl. Phys. B 403, 377 (1993), [hep-th/9210021].

[33] K. Sfetsos and D. C. Thompson, On non-abelian T-dual geometries with Ramond fluxes, Nucl. Phys. B 846 (2011) 21, arXiv:1012.1320.

[34] A. Karch and E. Katz, "Adding flavor to AdS / CFT", JHEP 0206, 043 (2002) [hepth/0205236].

[35] Y. Lozano and C. Nunez, Field theory aspects of non-Abelian T-duality and $\mathcal{N}=2$ linear quivers, JHEP 1605, 107 (2016), arXiv:1603.04440.

[36] Y. Lozano, N. T. Macpherson, J. Montero and C. Nunez, Three-dimensional N=4 Linear Quivers and non-Abelian T-duals, JHEP 1611, 133 (2016), arXiv:1609.09061.

[37] Y. Lozano, C. Nunez and S. Zacarias, BMN Vacua, Superstars and Non-Abelian Tduality, arXiv:1703.00417.

[38] G. Itsios, Y. Lozano, J. Montero and C. Nunez, The AdS5 non-Abelian T-dual of Klebanov-Witten as a $\mathcal{N}=1$ linear quiver from M5-branes, JHEP 1709, 038 (2017) arXiv: 1705.09661.

[39] H. Lin, O. Lunin and J. M. Maldacena, Bubbling AdS space and 1/2 BPS geometries, JHEP 0410, 025 (2004), arXiv:hep-th/0409174.

[40] H. Lin and J. M. Maldacena, Fivebranes from gauge theory, Phys. Rev. D 74, 084014 (2006), arXiv:hep-th/0509235.

[41] G. Ishiki, S. Shimasaki, Y. Takayama and A. Tsuchiya, Embedding of theories with SU(2-4) symmetry into the plane wave matrix model, JHEP 0611, 089 (2006), arXiv:hep-th/0610038. 
[42] Ö. Kelekci, Y. Lozano, N. T. Macpherson and E. Ó. Colgáin, Supersymmetry and nonAbelian T-duality in type II supergravity, Class. Quant. Grav. 32, no. 3, 035014 (2015), arXiv: 1409.7406.

[43] S. F. Hassan, T duality, space-time spinors and RR fields in curved backgrounds, Nucl. Phys. B 568, 145 (2000), arXiv:hep-th/9907152.

[44] Y. Lozano and N. T. Macpherson, A new AdS $S_{4} / C F T_{3}$ dual with extended SUSY and a spectral flow, JHEP 1411, 115 (2014), arXiv:1408.0912.

[45] Y. Lozano, E. O. O Colgáin and D. Rodríguez-Gómez, Hints of 5d Fixed Point Theories from Non-Abelian T-duality, JHEP 1405, 009 (2014), arXiv:1311.4842.

[46] R. C. Myers, Dielectric branes, JHEP 9912, 022 (1999), arXiv:hep-th/9910053.

[47] J. Polchinski and M. J. Strassler, The String dual of a confining four-dimensional gauge theory, arXiv:hep-th/0003136.

[48] N. Ishibashi, H. Kawai, Y. Kitazawa and A. Tsuchiya, A Large $N$ reduced model as superstring, Nucl. Phys. B 498, 467 (1997), arXiv:hep-th/9612115.

[49] M. M. Sheikh-Jabbari, Tiny graviton matrix theory: DLCQ of IIB plane-wave string theory, a conjecture, JHEP 0409, 017 (2004), arXiv: hep-th/0406214.

[50] Y. Lozano and D. Rodriguez-Gomez, Type II pp-wave matrix models from point-like gravitons, JHEP 0608, 022 (2006), arXiv:hep-th/0606057.

[51] M. Rocek and E. P. Verlinde, Nucl. Phys. B 373, 630 (1992) doi:10.1016/05503213(92)90269-H [hep-th/9110053].

[52] A. Giveon and D. Kutasov, Rev. Mod. Phys. 71, 983 (1999) doi:10.1103/RevModPhys.71.983 [hep-th/9802067].

[53] A. Giveon and M. Rocek, Nucl. Phys. B 380, 128 (1992) doi:10.1016/0550-3213(92)90518$\mathrm{G}$ [hep-th/9112070].

[54] D. Sadri and M. M. Sheikh-Jabbari, Giant hedgehogs: Spikes on giant gravitons, Nucl. Phys. B 687, 161 (2004), arXiv:hep-th/0312155.

[55] G. Bonelli, Matrix strings in pp wave backgrounds from deformed superYang-Mills theory, JHEP 0208, 022 (2002) arXiv:hep-th/0205213.

[56] B. Janssen and Y. Lozano, A Microscopical description of giant gravitons, Nucl. Phys. B 658, 281 (2003), arXiv:hep-th/0207199. 


\section{BIBLIOGRAPHY}

B. Janssen, Y. Lozano and D. Rodriguez-Gomez, A Microscopical description of giant gravitons II: The $A d S_{5} \times S^{5}$ background, Nucl. Phys. B 669, 363 (2003), arXiv:hep-th/0303183.

B. Janssen and Y. Lozano, On the dielectric effect for gravitational waves, Nucl. Phys. B 643, 399 (2002), arXiv:hep-th/0205254.

[57] A. Fayyazuddin and D. J. Smith, Localized intersections of M5-branes and four-dimensional superconformal field theories, JHEP 9904, 030 (1999), arXiv:hep-th/9902210.

[58] N. T. Macpherson, C. Núñez, L. A. Pando Zayas, V. G. J. Rodgers and C. A. Whiting, Type IIB supergravity solutions with $A d S_{5}$ from Abelian and non-Abelian $T$ dualities, JHEP 1502, 040 (2015), arXiv: 1410.2650.

[59] S. Ryu and T. Takayanagi, Phys. Rev. Lett. 96, 181602 (2006) doi:10.1103/PhysRevLett.96.181602 [hep-th/0603001].

[60] S. Ryu and T. Takayanagi, JHEP 0608, 045 (2006) doi:10.1088/1126-6708/2006/08/045 [hep-th/0605073].

[61] I. R. Klebanov, D. Kutasov and A. Murugan, Nucl. Phys. B 796, 274 (2008) doi:10.1016/j.nuclphysb.2007.12.017 [arXiv:0709.2140 [hep-th]].

[62] H. Ling, A. R. Mohazab, H. H. Shieh, G. van Anders and M. Van Raamsdonk, Little string theory from a double-scaled matrix model, JHEP 0610, 018 (2006), arXiv:hep-th/0606014.

[63] J. Polchinski, "Dirichlet Branes and Ramond-Ramond charges" Phys. Rev. Lett. 75, 4724 (1995) [hep-th/9510017].

[64] J. Polchinski, "Tasi lectures on D-branes" hep-th/9611050.

[65] H. Nastase, "Introduction to AdS-CFT" arXiv:0712.0689 [hep-th].

[66] E. S. Fradkin and A. A. Tseytlin, "Nonlinear Electrodynamics from Quantized Strings", Phys. Lett. 163B, 123 (1985).

[67] A. Abouelsaood, C. G. Callan, Jr., C. R. Nappi and S. A. Yost, "Open Strings in Background Gauge Fields", Nucl. Phys. B 280, 599 (1987).

[68] G. 't Hooft, “A Planar Diagram Theory for Strong Interactions”, Nucl. Phys. B 72, 461 (1974).

[69] S. S. Gubser, I. R. Klebanov and A. M. Polyakov, "Gauge theory correlators from noncritical string theory", Phys. Lett. B 428, 105 (1998) [hep-th/9802109]. 
[70] E. Witten, “Anti-de Sitter space and holography”, Adv. Theor. Math. Phys. 2, 253 (1998) [hep-th/9802150].

[71] O. Aharony, S. S. Gubser, J. M. Maldacena, H. Ooguri and Y. Oz, "Large N field theories, string theory and gravity", Phys. Rept. 323, 183 (2000) [hep-th/9905111].

[72] A. Hanany and E. Witten, "Type IIB superstrings, BPS monopoles, and threedimensional gauge dynamics", Nucl. Phys. B 492, 152 (1997) [hep-th/9611230].

[73] A. Hanany and A. Zaffaroni, "Branes and six-dimensional supersymmetric theories", Nucl. Phys. B 529, 180 (1998) [hep-th/9712145].

[74] S. Cremonesi and A. Tomasiello, "6d holographic anomaly match as a continuum limit", JHEP 1605, 031 (2016) [arXiv:1512.02225 [hep-th]].

[75] D. Gaiotto and A. Tomasiello, "Holography for $(1,0)$ theories in six dimensions", JHEP 1412, 003 (2014) [arXiv:1404.0711 [hep-th]].

[76] N. Bobev, G. Dibitetto, F. F. Gautason and B. Truijen, "Holography, Brane Intersections and Six-dimensional SCFTs", JHEP 1702, 116 (2017) [arXiv:1612.06324 [hep-th]].

[77] N. Seiberg and E. Witten, "Electric - magnetic duality, monopole condensation, and confinement in N=2 supersymmetric Yang-Mills theory", Nucl. Phys. B 426, 19 (1994) Erratum: [Nucl. Phys. B 430, 485 (1994)] [hep-th/9407087].

[78] D. Gaiotto, "N=2 dualities", JHEP 1208, 034 (2012) [arXiv:0904.2715 [hep-th]].

[79] D. Gaiotto and J. Maldacena, "The Gravity duals of N=2 superconformal field theories", JHEP 1210, 189 (2012) [arXiv:0904.4466 [hep-th]].

[80] R. A. Reid-Edwards and B. Stefanski, jr., "On Type IIA geometries dual to $N=2$ SCFTs", Nucl. Phys. B 849, 549 (2011) [arXiv:1011.0216 [hep-th]].

[81] O. Aharony, L. Berdichevsky and M. Berkooz, “4d N=2 superconformal linear quivers with type IIA duals", JHEP 1208, 131 (2012) [arXiv:1206.5916 [hep-th]].

[82] J. van Gorsel and S. Zacarías, "A Type IIB Matrix Model via non-Abelian T-dualities", JHEP 12, 101 (2017) [arXiv:1711.03419 [hep-th]].

[83] E. Alvarez, L. Alvarez-Gaume and Y. Lozano, "A Canonical approach to duality transformations", Phys. Lett. B 336, 183 (1994) [hep-th/9406206].

[84] K. Sfetsos and D. C. Thompson, "Spacetimes for $\lambda$-deformations", JHEP 1412, 164 (2014) [arXiv:1410.1886 [hep-th]]. 
[85] D. Gaiotto and E. Witten, "S-Duality of Boundary Conditions In N=4 Super Yang-Mills Theory" Adv. Theor. Math. Phys. 13, no. 3, 721 (2009) [arXiv:0807.3720 [hep-th]].

[86] E. D'Hoker, J. Estes and M. Gutperle, "Exact half-BPS Type IIB interface solutions. I. Local solution and supersymmetric Janus", JHEP 0706, 021 (2007) [arXiv:0705.0022 [hep-th]].

[87] E. D'Hoker, J. Estes and M. Gutperle, "Exact half-BPS Type IIB interface solutions. II. Flux solutions and multi-Janus”, JHEP 0706, 022 (2007) [arXiv:0705.0024 [hep-th]].

[88] B. Assel, C. Bachas, J. Estes and J. Gomis, "Holographic Duals of D=3 N=4 Superconformal Field Theories", JHEP 1108, 087 (2011) [arXiv:1106.4253 [hep-th]].

[89] O. Aharony, L. Berdichevsky, M. Berkooz and I. Shamir, "Near-horizon solutions for D3branes ending on 5-branes", Phys. Rev. D 84, 126003 (2011) [arXiv:1106.1870 [hep-th]].

[90] Y. Lozano, N. T. Macpherson, J. Montero and C. Nunez, "Three-dimensional $\mathcal{N}=4$ linear quivers and non-Abelian T-duals", JHEP 1611, 133 (2016) [arXiv:1609.09061 [hepth]].

[91] K. Sfetsos and D. C. Thompson, "On non-abelian T-dual geometries with Ramond fluxes", Nucl. Phys. B 846, 21 (2011) [arXiv:1012.1320 [hep-th]].

[92] C. Nunez, D. Roychowdhury and D. C. Thompson, "Integrability and non-integrability in $\mathcal{N}=2$ SCFTs and their holographic backgrounds", JHEP 1807, 044 (2018) [arXiv:1804.08621 [hep-th]].

[93] Y. Lozano and C. Nunez, "Field theory aspects of non-Abelian T-duality and $\mathcal{N}=2$ linear quivers", JHEP 1605, 107 (2016) [arXiv:1603.04440 [hep-th]].

[94] O. Aharony, A. Hanany and B. Kol, "Webs of $(p, q)$ five-branes, five-dimensional field theories and grid diagrams", JHEP 9801, 002 (1998) [hep-th/9710116].

[95] O. Aharony and A. Hanany, "Branes, superpotentials and superconformal fixed points", Nucl. Phys. B 504, 239 (1997) [hep-th/9704170].

[96] K. A. Intriligator, D. R. Morrison and N. Seiberg, "Five-dimensional supersymmetric gauge theories and degenerations of Calabi-Yau spaces", Nucl. Phys. B 497, 56 (1997) [hep-th/9702198].

[97] O. Bergman and G. Zafrir, "5d fixed points from brane webs and O7-planes", JHEP 1512, 163 (2015) [arXiv:1507.03860 [hep-th]].

[98] E. D'Hoker, M. Gutperle, A. Karch and C. F. Uhlemann, "Warped $A d S_{6} \times S^{2}$ in Type IIB supergravity I: Local solutions", JHEP 1608, 046 (2016) [arXiv:1606.01254 [hep-th]]. 
[99] E. D'Hoker, M. Gutperle and C. F. Uhlemann, "Warped $A d S_{6} \times S^{2}$ in Type IIB supergravity II: Global solutions and five-brane webs", JHEP 1705, 131 (2017) [arXiv:1703.08186 [hep-th]].

[100] E. D'Hoker, M. Gutperle and C. F. Uhlemann, "Warped $A d S_{6} \times S^{2}$ in Type IIB supergravity III: Global solutions with seven-branes", JHEP 1711, 200 (2017) [arXiv:1706.00433 [hep-th]].

[101] Y. Lozano, N. T. Macpherson and J. Montero, "AdS6 T-duals and type IIB AdS6× $S^{2}$ geometries with 7-branes", JHEP 1901, 116 (2019) [arXiv:1810.08093 [hep-th]].

[102] E. D'Hoker, M. Gutperle and C. F. Uhlemann, "Holographic duals for five-dimensional superconformal quantum field theories", Phys. Rev. Lett. 118, no. 10, 101601 (2017) [arXiv:1611.09411 [hep-th]].

[103] Y. Lozano, N. T. Macpherson, C. Nunez and A. Ramirez, "Two dimensional $\mathcal{N}=(0,4)$ quivers dual to $A d S_{3}$ solutions in massive IIA", arXiv:1909.10510 [hep-th].

[104] Y. Lozano, N. T. Macpherson, C. Nunez and A. Ramirez, "1/4 BPS AdS $S_{3} / C F T_{2}$ " arXiv:1909.09636 [hep-th].

[105] Y. Lozano, N. T. Macpherson, C. Nunez and A. Ramirez, "AdS $S_{3}$ solutions in Massive IIA with small $\mathcal{N}=(4,0)$ supersymmetry", arXiv:1908.09851 [hep-th].

[106] K. Filippas, C. Nunez and J. van Gorsel, "Integrability and holographic aspects of six-dimensional $\mathcal{N}=(1,0)$ superconformal field theories", JHEP 1906, 069 (2019) [arXiv:1901.08598 [hep-th]].

[107] J. Van Gorsel, "Infinite Linear Quivers and Continuous Rank Functions", arXiv:1911.06807 [hep-th].

[108] E. Witten, "String theory dynamics in various dimensions", Nucl. Phys. B 443, 85 (1995) [hep-th/9503124].

[109] N. Seiberg and E. Witten, "Comments on string dynamics in six-dimensions", Nucl. Phys. B 471, 121 (1996) [hep-th/9603003].

[110] J. D. Blum and K. A. Intriligator, "New phases of string theory and 6-D RG fixed points via branes at orbifold singularities", Nucl. Phys. B 506, 199 (1997) [hep-th/9705044].

[111] N. Seiberg, "Nontrivial fixed points of the renormalization group in six-dimensions", Phys. Lett. B 390, 169 (1997) [hep-th/9609161]. 
[112] U. H. Danielsson, G. Ferretti, J. Kalkkinen and P. Stjernberg, "Notes on supersymmetric gauge theories in five-dimensions and six-dimensions", Phys. Lett. B 405, 265 (1997) [hep-th/9703098].

[113] A. Hanany and A. Zaffaroni, "Branes and six-dimensional supersymmetric theories", Nucl. Phys. B 529, 180 (1998) [hep-th/9712145].

[114] I. Brunner and A. Karch, "Branes at orbifolds versus Hanany Witten in six-dimensions", JHEP 9803, 003 (1998) [hep-th/9712143].

[115] K. Ohmori, H. Shimizu and Y. Tachikawa, "Anomaly polynomial of E-string theories", JHEP 1408, 002 (2014) [arXiv:1404.3887 [hep-th]].

[116] M. Del Zotto, J. J. Heckman, A. Tomasiello and C. Vafa, "6d Conformal Matter", JHEP 1502, 054 (2015) [arXiv:1407.6359 [hep-th]].

[117] C. Cordova, T. T. Dumitrescu and K. Intriligator, "Anomalies, renormalization group flows, and the a-theorem in six-dimensional (1, 0) theories", JHEP 1610, 080 (2016) [arXiv:1506.03807 [hep-th]].

[118] K. Intriligator, " $6 d, \mathcal{N}=(1,0)$ Coulomb branch anomaly matching", JHEP 1410, 162 (2014) [arXiv:1408.6745 [hep-th]].

[119] K. Ohmori, H. Shimizu, Y. Tachikawa and K. Yonekura, "Anomaly polynomial of general 6d SCFTs", PTEP 2014, no. 10, 103B07 (2014) [arXiv:1408.5572 [hep-th]].

[120] M. Beccaria and A. A. Tseytlin, "Conformal anomaly c-coefficients of superconformal 6d theories", JHEP 1601, 001 (2016) [arXiv:1510.02685 [hep-th]].

[121] L. Bhardwaj, "Classification of $6 d \mathcal{N}=(1,0)$ gauge theories", JHEP 1511, 002 (2015) [arXiv:1502.06594 [hep-th]].

[122] I. Bandos, H. Samtleben and D. Sorokin, "Duality-symmetric actions for non-Abelian tensor fields", Phys. Rev. D 88, no. 2, 025024 (2013) [arXiv:1305.1304 [hep-th]].

[123] C. M. Chang, "5d and 6d SCFTs Have No Weak Coupling Limit", arXiv:1810.04169 [hep-th].

[124] F. Apruzzi, M. Fazzi, A. Passias, D. Rosa and A. Tomasiello, "AdS 6 solutions of type II supergravity”, JHEP 1411, 099 (2014) Erratum: [JHEP 1505, 012 (2015)] [arXiv:1406.0852 [hep-th]].

[125] F. Apruzzi, M. Fazzi, A. Passias, A. Rota and A. Tomasiello, "Six-Dimensional Superconformal Theories and their Compactifications from Type IIA Supergravity", Phys. Rev. Lett. 115, no. 6, 061601 (2015) [arXiv:1502.06616 [hep-th]]. 
[126] A. Passias, A. Rota and A. Tomasiello, "Universal consistent truncation for 6d/7d gauge/gravity duals", JHEP 1510, 187 (2015) [arXiv:1506.05462 [hep-th]].

[127] F. Apruzzi and M. Fazzi, "AdS $S_{7} / C F T_{6}$ with orientifolds", JHEP 1801, 124 (2018) [arXiv:1712.03235 [hep-th]].

[128] N. T. Macpherson and A. Tomasiello, "Minimal flux Minkowski classification", JHEP 1709, 126 (2017) [arXiv:1612.06885 [hep-th]].

[129] A. Hanany and E. Witten, "Type IIB superstrings, BPS monopoles, and threedimensional gauge dynamics", Nucl. Phys. B 492, 152 (1997) [hep-th/9611230].

[130] W. Nahm, "Supersymmetries and their Representations", Nucl. Phys. B 135, 149 (1978). S. Minwalla, "Restrictions imposed by superconformal invariance on quantum field theories", Adv. Theor. Math. Phys. 2, 783 (1998) [hep-th/9712074].

[131] H. Samtleben, E. Sezgin and R. Wimmer, "(1,0) superconformal models in six dimensions", JHEP 1112, 062 (2011) [arXiv:1108.4060 [hep-th]].

[132] K. Filippas, "Non-integrability on $A d S_{3}$ supergravity backgrounds", JHEP 2002, 027 (2020) [arXiv:1910.12981 [hep-th]].

[133] F. Bigazzi, R. Casero, A. L. Cotrone, E. Kiritsis and A. Paredes, "Non-critical holography and four-dimensional CFT's with fundamentals", JHEP 0510, 012 (2005) [hepth/0505140].

[134] R. Casero, C. Nunez and A. Paredes, "Towards the string dual of N=1 SQCD-like theories", Phys. Rev. D 73, 086005 (2006) [hep-th/0602027].

[135] C. Nunez, D. Roychowdhury, S. Speziali and S. Zacarias, "Holographic Aspects of Four Dimensional $\mathcal{N}=2$ SCFTs and their Marginal Deformations", arXiv:1901.02888 [hepth].

[136] C. Nunez, J. M. Penin, D. Roychowdhury and J. van Gorsel, "The non-Integrability of Strings in Massive Type IIA and their Holographic duals", JHEP 1806, 078 (2018) [arXiv:1802.04269 [hep-th]].

[137] U. Kol, C. Nunez, D. Schofield, J. Sonnenschein and M. Warschawski, "Confinement, Phase Transitions and non-Locality in the Entanglement Entropy", JHEP 1406, 005 (2014) [arXiv:1403.2721 [hep-th]].

N. T. Macpherson, C. Nunez, L. A. Pando Zayas, V. G. J. Rodgers and C. A. Whiting, "Type IIB supergravity solutions with AdS5 from Abelian and non-Abelian T dualities", JHEP 1502, 040 (2015) [arXiv:1410.2650 [hep-th]]. 
[138] P. Basu and L. A. Pando Zayas, "Chaos rules out integrability of strings on $A d S_{5} \times T^{1,1}$ " Phys. Lett. B 700, 243 (2011) [arXiv:1103.4107 [hep-th]].

[139] P. Basu and L. A. Pando Zayas, "Analytic Non-integrability in String Theory", Phys. Rev. D 84, 046006 (2011) [arXiv:1105.2540 [hep-th]].

[140] C. Nunez, D. Roychowdhury and D. C. Thompson, "Integrability and non-integrability in $\mathcal{N}=2$ SCFTs and their holographic backgrounds", JHEP 1807, 044 (2018) [arXiv:1804.08621 [hep-th]].

[141] K. Sfetsos, "Integrable interpolations: From exact CFTs to non-Abelian T-duals", Nucl. Phys. B 880, 225 (2014) [arXiv:1312.4560 [hep-th]].

[142] K. Sfetsos and D. C. Thompson, "Spacetimes for $\lambda$-deformations", JHEP 1412, 164 (2014) [arXiv:1410.1886 [hep-th]].

S. Driezen, A. Sevrin and D. C. Thompson, "D-branes in $\lambda$-deformations", JHEP 1809, 015 (2018) [arXiv:1806.10712 [hep-th]].

[143] K. Sfetsos and D. C. Thompson, "On non-abelian T-dual geometries with Ramond fluxes", Nucl. Phys. B 846, 21 (2011) [arXiv:1012.1320 [hep-th]].

[144] Y. Lozano and C. Nunez, "Field theory aspects of non-Abelian T-duality and $\mathcal{N}=2$ linear quivers", JHEP 1605, 107 (2016) [arXiv:1603.04440 [hep-th]].

[145] O. Lunin and J. M. Maldacena, "Deforming field theories with $U(1) \times U(1)$ global symmetry and their gravity duals", JHEP 0505, 033 (2005) [hep-th/0502086].

[146] L. Wulff, "Condition on Ramond-Ramond fluxes for factorization of worldsheet scattering in anti-de Sitter space", Phys. Rev. D 96, no. 10, 101901 (2017) [arXiv:1708.09673 [hepth]].

[147] L. Wulff, "Constraining integrable AdS/CFT with factorized scattering", JHEP 1904, 133 (2019) [arXiv:1903.08660 [hep-th]].

[148] J. M. Maldacena, "Wilson loops in large $N$ field theories", Phys. Rev. Lett. 80, 4859 (1998) [hep-th/9803002].

[149] A. V. Ramallo, "Introduction to the AdS/CFT correspondence", Springer Proc. Phys. 161, 411-474 (2015) [arXiv:1310.4319 [hep-th]].

[150] C. Nunez, A. Paredes and A. V. Ramallo, "Unquenched Flavor in the Gauge/Gravity Correspondence", Adv. High Energy Phys. 2010, 196714 (2010) [arXiv:1002.1088 [hep$\operatorname{th}]$. 
R. Casero, C. Nunez and A. Paredes, "Towards the string dual of N=1 SQCD-like theories", Phys. Rev. D 73, 086005 (2006) [hep-th/0602027].

P. Koerber and D. Tsimpis, "Supersymmetric sources, integrability and generalizedstructure compactifications", JHEP 0708, 082 (2007) [arXiv:0706.1244 [hep-th]].

A. Barranco, J. Gaillard, N. T. Macpherson, C. Nunez and D. C. Thompson, "G-structures and Flavouring non-Abelian T-duality", JHEP 1308, 018 (2013) [arXiv:1305.7229 [hep-th]].

[151] J. J. Kovacic, "An algorithm for solving second order linear homogeneous differential equations", J. Symbolic Computation (1986) 2, 3-43.

[152] K. Zarembo, "Integrability in Sigma-Models", arXiv:1712.07725 [hep-th].

[153] G. Arutyunov and S. Frolov, "Integrable Hamiltonian for classical strings on $A d S_{5} \times S^{5}$ ", JHEP 0502, 059 (2005) [hep-th/0411089].

[154] N. Seiberg, "Nontrivial fixed points of the renormalization group in six-dimensions", Phys. Lett. B 390, 169 (1997) [hep-th/9609161].

[155] A. Hanany and E. Witten, "Type IIB superstrings, BPS monopoles, and threedimensional gauge dynamics", Nucl. Phys. B 492, 152 (1997) [hep-th/9611230].

[156] A. Hanany and A. Zaffaroni, "Chiral symmetry from type IIA branes", Nucl. Phys. B 509, 145 (1998) [hep-th/9706047].

I. Brunner and A. Karch, "Branes and six-dimensional fixed points", Phys. Lett. B 409, 109 (1997) [hep-th/9705022].

[157] F. Apruzzi, M. Fazzi, D. Rosa and A. Tomasiello, "All AdS $S_{7}$ solutions of type II supergravity", JHEP 1404, 064 (2014) [arXiv:1309.2949 [hep-th]].

[158] A. Passias, A. Rota and A. Tomasiello, "Universal consistent truncation for 6d/7d gauge/gravity duals", JHEP 1510, 187 (2015) [arXiv:1506.05462 [hep-th]].

[159] F. Apruzzi, G. Dibitetto and L. Tizzano, "A new 6d fixed point from holography", [arXiv:1603.06576 [hep-th]].

A. Rota and A. Tomasiello, "AdS $S_{4}$ compactifications of AdS $S_{7}$ solutions in type II supergravity", [arXiv:1502.06622 [hep-th]].

F. Apruzzi, M. Fazzi, A. Passias and A. Tomasiello, "Supersymmetric Ad $S_{5}$ solutions of massive IIA supergravity", [arXiv:1502.06620 [hep-th]].

A. Passias and A. Tomasiello, "Spin-2 spectrum of six-dimensional field theories", [arXiv:1604.04286 [hep-th]]. 
G. Dibitetto and N. Petri, "BPS objects in $D=7$ supergravity and their $M$-theory origin", [arXiv:1707.06152 [hep-th]].

G. Dibitetto and N. Petri, "6d surface defects from massive type IIA", arXiv:1707.06154 [hep-th].

[160] F. Apruzzi and M. Fazzi, "AdS $S_{7} / C F T_{6}$ with orientifolds", JHEP 1801, 124 (2018) [arXiv:1712.03235 [hep-th]].

[161] E. Ott, "Chaos in Dynamical Systems" Cambridge: Cambridge University Press doi:10.1017/CBO9780511803260

[162] P. Basu and L. A. Pando Zayas, "Chaos rules out integrability of strings on $A d S_{5} \times T^{1,1}$ ", Phys. Lett. B 700, 243 (2011) [arXiv:1103.4107 [hep-th]].

[163] P. Basu and L. A. Pando Zayas, "Analytic Non-integrability in String Theory", Phys. Rev. D 84, 046006 (2011) [arXiv:1105.2540 [hep-th]].

[164] U. H. Danielsson, G. Ferretti, J. Kalkkinen and P. Stjernberg, "Notes on supersymmetric gauge theories in five-dimensions and six-dimensions", Phys. Lett. B 405, 265 (1997) [hep-th/9703098].

[165] M. Fazzi, "Higher-dimensional field theories from type II supergravity", arXiv:1712.04447 [hep-th].

[166] I. R. Klebanov, D. Kutasov and A. Murugan, "Entanglement as a probe of confinement", Nucl. Phys. B 796, 274 (2008) [arXiv:0709.2140 [hep-th]].

For a refinement of these formulas see N. T. Macpherson, C. Núñez, L. A. Pando Zayas, V. G. J. Rodgers and C. A. Whiting, "Type IIB supergravity solutions with AdS $S_{5}$ from Abelian and non-Abelian T dualities", JHEP 1502, 040 (2015) [arXiv:1410.2650 [hep-th]] and

Y. Bea, J. D. Edelstein, G. Itsios, K. S. Kooner, C. Nunez, D. Schofield and J. A. SierraGarcia, "Compactifications of the Klebanov-Witten CFT and new AdS $S_{3}$ backgrounds", JHEP 1505, 062 (2015) [arXiv:1503.07527 [hep-th]].

[167] L. A. Pando Zayas and C. A. Terrero-Escalante, "Chaos in the Gauge / Gravity Correspondence", JHEP 1009 (2010) 094 [arXiv:1007.0277 [hep-th]].

[168] P. Basu, D. Das and A. Ghosh, "Integrability Lost", Phys. Lett. B 699 (2011) 388 [arXiv:1103.4101 [hep-th]].

[169] D. Giataganas, L. A. Pando Zayas and K. Zoubos, "On Marginal Deformations and Non-Integrability", JHEP 1401 (2014) 129 [arXiv:1311.3241 [hep-th]]. 
[170] D. Giataganas and K. Zoubos, "Non-integrability and Chaos with Unquenched Flavor", JHEP 1710, 042 (2017) [arXiv:1707.04033 [hep-th]].

[171] A. Stepanchuk and A. A. Tseytlin, "On (non)integrability of classical strings in p-brane backgrounds", J. Phys. A 46 (2013) 125401 [arXiv:1211.3727 [hep-th]].

[172] Y. Asano, D. Kawai and K. Yoshida, "Chaos in the BMN matrix model", JHEP 1506 (2015) 191 [arXiv:1503.04594 [hep-th]].

[173] Y. Asano, D. Kawai, H. Kyono and K. Yoshida, "Chaotic strings in a near Penrose limit of $A d S_{5} \times T^{1,1}$, JHEP 1508 (2015) 060 [arXiv:1505.07583 [hep-th]].

[174] T. Ishii, K. Murata and K. Yoshida, "Fate of chaotic strings in a confining geometry", Phys. Rev. D 95 (2017) no.6, 066019 [arXiv:1610.05833 [hep-th]].

[175] K. L. Panigrahi and M. Samal, "Chaos in classical string dynamics in $\hat{\gamma}$ deformed $A d S_{5} \times$ $T^{1,1}$, Phys. Lett. B 761, 475 (2016) [arXiv:1605.05638 [hep-th]].

[176] D. Giataganas and K. Sfetsos, "Non-integrability in non-relativistic theories", JHEP 1406, 018 (2014) [arXiv:1403.2703 [hep-th]].

[177] P. Basu, D. Das, A. Ghosh and L. A. Pando Zayas, "Chaos around Holographic Regge Trajectories", JHEP 1205, 077 (2012) [arXiv:1201.5634 [hep-th]].

[178] X. Bai, B. H. Lee, T. Moon and J. Chen, "Chaos in Lifshitz Spacetimes", J. Korean Phys. Soc. 68, no. 5, 639 (2016) [arXiv:1406.5816 [hep-th]].

[179] Y. Chervonyi and O. Lunin, "(Non)-Integrability of Geodesics in D-brane Backgrounds", JHEP 1402, 061 (2014) [arXiv:1311.1521 [hep-th]].

[180] J. R. David and A. Sadhukhan, "Classical integrability in the BTZ black hole", JHEP 1108, 079 (2011) [arXiv:1105.0480 [hep-th]].

[181] D. Roychowdhury, "Analytic integrability for strings on $\eta$ and $\lambda$ deformed backgrounds", JHEP 1710 (2017) 056 [arXiv:1707.07172 [hep-th]].

[182] Jerald Kovacic. Journal of Symbolic Computation (1986) 2,3-43.

[183] Marco Sandri, "6d holographic anomaly match as a continuum limit", The Mathematical Journal, volume 6, issue 3, pages 78-84, Miller Freedman Publications (1996).

[184] G. Itsios, H. Nastase, C. Nunez, K. Sfetsos and S. Zacarias, "Penrose limits of Abelian and non-Abelian T-duals of $A d S_{5} \times S^{5}$ and their field theory duals", JHEP 1801, 071 (2018) [arXiv:1711.09911 [hep-th]]. 
[185] J. Maldacena, S. H. Shenker and D. Stanford, "A bound on chaos", JHEP 1608, 106 (2016) [arXiv:1503.01409 [hep-th]].

[186] J. de Boer, E. Llabrés, J. F. Pedraza and D. Vegh, "Chaotic strings in AdS/CFT", arXiv:1709.01052 [hep-th].

[187] S. A. Hartnoll and C. Nunez, "Rotating membranes on G(2) manifolds, logarithmic anomalous dimensions and N=1 duality", JHEP 0302, 049 (2003) [hep-th/0210218].

[188] Oseledec, V.I. 1968. "A multiplicative ergodic theorem: Lyapunov characteristic numbers for dynamical systems", Trans. Moscow Math. Soc. 19:197-231.

[189] Benettin, G., L. Galgani, A. Giorgilli, and J. M. Strelcyn. 1980. "Lyapunov characteristic exponents for smooth dynamical systems and for Hamiltonian systems: A method for computing all of them. Part 2: Numerical application", Meccanica 15:21-30.

[190] K. Sfetsos and D. C. Thompson, "On non-abelian T-dual geometries with Ramond fluxes", Nucl. Phys. B 846, 21 (2011) [arXiv:1012.1320 [hep-th]].

[191] B. Assel, C. Bachas, J. Estes and J. Gomis, "IIB Duals of D=3 N=4 Circular Quivers", JHEP 1212, 044 (2012) [arXiv:1210.2590 [hep-th]].

[192] Y. Lozano, E. O Colgain, D. Rodriguez-Gomez and K. Sfetsos, "Supersymmetric AdS 6 via T Duality", Phys. Rev. Lett. 110, no. 23, 231601 (2013) [arXiv:1212.1043 [hep-th]].

[193] A. Brandhuber and Y. Oz, "The D-4 - D-8 brane system and five-dimensional fixed points", Phys. Lett. B 460 (1999) 307 [hep-th/9905148].

[194] Y. Lozano, N. T. Macpherson and J. Montero, "AdS $S_{6}$ T-duals and type IIB AdS $S_{6} \times S^{2}$ geometries with 7-branes", JHEP 1901, 116 (2019) [arXiv:1810.08093 [hep-th]].

[195] Y. Lozano, N. T. Macpherson and J. Montero, "AdS $S_{6} T$-duals and $A d S_{6} \times S^{2}$ geometries in Type IIB", PoS CORFU 2018, 122 (2019) [arXiv:1811.00054 [hep-th]].

[196] G. Itsios, C. Nunez, K. Sfetsos and D. C. Thompson, "On Non-Abelian T-Duality and new N=1 backgrounds", Phys. Lett. B 721, 342 (2013) [arXiv:1212.4840 [hep-th]].

G. Itsios, C. Nunez, K. Sfetsos and D. C. Thompson, "Non-Abelian T-duality and the AdS/CFT correspondence:new N=1 backgrounds", Nucl. Phys. B 873, 1 (2013) [arXiv:1301.6755 [hep-th]].

Y. Lozano, E. O. O Colgáin and D. Rodriguez-Gomez, "Hints of 5d Fixed Point Theories from Non-Abelian T-duality", JHEP 1405, 009 (2014) [arXiv:1311.4842 [hep-th]].

Y. Lozano, N. T. Macpherson, J. Montero and E. O. Colgain, "New $A d S_{3} \times S^{2}$ T-duals with $\mathcal{N}=(0,4)$ supersymmetry", JHEP 1508, 121 (2015) [arXiv:1507.02659 [hep-th]]. 
[197] Y. Lozano and C. Nunez, "Field theory aspects of non-Abelian T-duality and $\mathcal{N}=2$ linear quivers", JHEP 1605, 107 (2016) [arXiv:1603.04440 [hep-th]].

[198] Y. Lozano, N. T. Macpherson, J. Montero and C. Nunez, "Three-dimensional $\mathcal{N}=4$ linear quivers and non-Abelian T-duals", JHEP 1611, 133 (2016) [arXiv:1609.09061 [hepth]].

Y. Lozano, C. Nunez and S. Zacarias, "BMN Vacua, Superstars and Non-Abelian Tduality", JHEP 1709, 000 (2017) [arXiv:1703.00417 [hep-th]].

G. Itsios, Y. Lozano, J. Montero and C. Nunez, "The AdS $S_{5}$ non-Abelian T-dual of Klebanov-Witten as a $\mathcal{N}=1$ linear quiver from M5-branes", JHEP 1709, 038 (2017) [arXiv:1705.09661 [hep-th]].

[199] K. G. Wilson, "Confinement of Quarks", Phys. Rev. D 10, 2445 (1974).

[200] J. M. Maldacena, "Wilson loops in large $N$ field theories", Phys. Rev. Lett. 80, 4859 (1998) [hep-th/9803002]. 